J. Inst. Math. Jussieu (2023), 22(2), 799-878

doi:10.1017/S1474748021000293 C The Author(s), 2021. Published by Cambridge University

Press. This is an Open Access article, distributed under the terms of the Creative Commons

Attribution-NonCommercial-NoDerivatives licence (http://creativecommons.org/licenses/

by-nc-nd/4.0), which permits non-commercial re-use, distribution, and reproduction in any medium,

provided that no alterations are made and the original article is properly cited. The written permission of Cambridge University Press must be obtained prior to any commercial use and/or adaptation of the article.

\title{
THE GENERIC FIBRE OF MODULI SPACES OF BOUNDED LOCAL $G$-SHTUKAS
}

\author{
URS HARTL (D) ${ }^{1}$ AND EVA VIEHMANN ${ }^{2}$ \\ ${ }^{1}$ Universität Münster, Mathematisches Institut, Einsteinstr. 62, D - 48149 Münster, \\ Germany \\ (www.math.uni-muenster.de/u/urs.hartl/) \\ 2 Technische Universität München, Fakultät für Mathematik-M11, Boltzmannstr. 3, \\ D - 85748 Garching b. München, Germany
}

(Received 12 March 2020; revised 26 May 2021; accepted 28 May 2021; first published online 12 July 2021)

\begin{abstract}
Moduli spaces of bounded local $G$-shtukas are a group-theoretic generalisation of the function field analogue of Rapoport and Zink's moduli spaces of $p$-divisible groups. In this article we generalise some very prominent concepts in the theory of Rapoport-Zink spaces to our setting. More precisely, we define period spaces, as well as the period map from a moduli space of bounded local $G$-shtukas to the corresponding period space, and we determine the image of the period map. Furthermore, we define a tower of coverings of the generic fibre of the moduli space, which is equipped with a Hecke action and an action of a suitable automorphism group. Finally, we consider the $\ell$-adic cohomology of these towers.

Les espaces de modules de $G$-chtoucas locaux bornés sont une généralisation des espaces de modules de groupes $p$-divisibles de Rapoport-Zink, au cas d'un corps de fonctions local, pour des groupes plus généraux et des copoids pas nécessairement minuscules. Dans cet article nous définissons les espaces de périodes et l'application de périodes associés à un tel espace, et nous calculons son image. Nous étudions la tour au-dessus de la fibre générique de l'espace de modules, équipée d'une action de Hecke ainsi que d'une action d'un groupe d'automorphismes. Enfin, nous définissons la cohomologie $\ell$-adique de ces tours.
\end{abstract}

Keywords: local G-shtukas, Rapoport-Zink spaces, period domains, period morphism, cohomology

2020 Mathematics Subject Classification: 20G25, 11G09, 14L05, 14M15

\section{Contents}

1 Introduction

2 Bounded local $G$-shtukas

3 Rapoport-Zink spaces for bounded local $G$-shtukas 
5 Local systems of $\mathbb{F}_{q}((z))$-vector spaces

6 The period morphisms

7 The tower of étale coverings

8 The image of the period morphism

9 Cohomology

\section{Introduction}

Towers of moduli spaces of $p$-divisible groups with additional structure as defined by Drinfeld [35] and Rapoport and Zink [80] have become a central topic in the study of the geometric realisation of local Langlands correspondences. These towers consist of covering spaces of the generic fibre of moduli spaces of $p$-divisible groups with EL or PEL structure. They carry a Hecke action and an action of an associated automorphism group of the defining $p$-divisible group with extra structure and possess a period morphism to a $p$-adic period space. Recently, generalisations of these moduli spaces to groups of unramified Hodge type (instead of PEL type) have been defined by Kim [69] and Howard and Pappas [62]. Conjecturally, in all of these cases, the cohomology of the tower realises local Langlands correspondences. Several cases of these conjectures have been shown so far; compare, for example, [36], [27]. However, in general, still very little is known.

In the present article we define the analogous towers, cohomology groups and period spaces in the function field case and study their basic properties. This generalises Drinfeld's work [35]. We thus provide the foundations for a theory similar to the one initiated by Drinfeld, Rapoport and Zink. It is conceivable that the cohomology of our towers likewise realises local Langlands correspondences. For Drinfeld's towers [35] this was conjectured by Carayol [26] and proved by Boyer [23] and Hausberger [59], building on work of Laumon, Rapoport and Stuhler [73]. One major difference in our context is that instead of being restricted to groups of PEL or Hodge type, there is a natural and group-theoretic way to define moduli spaces of local $G$-shtukas for any reductive group $G$. Furthermore, one can define more general boundedness conditions than minuscule bounds which would be the direct analogue of the number field situation.

To give an overview of their definition, let $\mathbb{F}_{q}$ be a finite field with $q$ elements, let $\mathbb{F}$ be a fixed algebraic closure of $\mathbb{F}_{q}$ and let $\mathbb{F}_{q} \llbracket z \rrbracket$ and $\mathbb{F}_{q} \llbracket \zeta \rrbracket$ be the power series rings over $\mathbb{F}_{q}$ in the (independent) variables $z$, respectively $\zeta$. As base schemes we will consider the category $\mathcal{N} i l p_{\mathbb{F}_{q} \llbracket \zeta \rrbracket}$ consisting of schemes over $\operatorname{Spec} \mathbb{F}_{q} \llbracket \zeta \rrbracket$ on which $\zeta$ is locally nilpotent. Let $G$ be a parahoric group scheme over $\operatorname{Spec} \mathbb{F}_{q} \llbracket z \rrbracket$ in the sense of [25, Définition 5.2.6] and [48] with connected reductive generic fibre. (One may ask whether the assumptions on $G$ can be relaxed, but we crucially use the ind-projectivity of $\mathcal{F} \ell_{G}$ in the central Propositions 2.6 and 7.8; see the beginning of Section 2 for more explanations.)

Let $S \in \mathcal{N} i l p_{\mathbb{F}_{q} \llbracket \zeta \rrbracket}$ and let $H$ be a sheaf of groups on $S$ for the fpqc topology. By a (right) $H$-torsor on $S$ we mean a sheaf $\mathcal{H}$ for the fpqc topology on $S$ together with a 
(right) action of the sheaf $H$ such that $\mathcal{H}$ is isomorphic to $H$ on an fpqc covering of $S$. Here $H$ is viewed as an $H$-torsor by right multiplication. Let $L G$ and $L^{+} G$ be the loop group and the group of positive loops associated with $G$; compare Section 2. Let $\mathcal{G}$ be an $L^{+} G$-torsor on $S$. Via the inclusion of sheaves $L^{+} G \subset L G$ we can associate an $L G$-torsor $L \mathcal{G}$ with $\mathcal{G}$. Also, for an $L G$-torsor $\mathcal{G}$ on $S$ we denote by $\sigma^{*} \mathcal{G}$ the pullback of $\mathcal{G}$ under the $q$-Frobenius morphism $\sigma:=\operatorname{Frob}_{q}: S \rightarrow S$.

Definition 1.1. A local $G$-shtuka over some $S \in \mathcal{N} i l p_{\mathbb{F}_{q} \llbracket \zeta \rrbracket}$ is a pair $\underline{\mathcal{G}}=\left(\mathcal{G}, \tau_{\mathcal{G}}\right)$ consisting of an $L^{+} G$-torsor $\mathcal{G}$ on $S$ and an isomorphism of the associated $L G$-torsors $\tau_{\mathcal{G}}: \sigma^{*} L \mathcal{G} \stackrel{\sim}{\longrightarrow}$ $L \mathcal{G}$.

A quasi-isogeny $g:\left(\mathcal{G}^{\prime}, \tau_{\mathcal{G}^{\prime}}\right) \rightarrow\left(\mathcal{G}, \tau_{\mathcal{G}}\right)$ between local $G$-shtukas over $S$ is an isomorphism $g: L \mathcal{G}^{\prime} \stackrel{\sim}{\longrightarrow} L$ of the associated $L G$-torsors with $g \circ \tau_{\mathcal{G}^{\prime}}=\tau_{\mathcal{G}} \circ \sigma^{*} g$.

Local $G$-shtukas were introduced and studied in [56], [57] in the case where $G$ is a constant split reductive group over $\mathbb{F}_{q}$ and in [41], [50] for $G=\mathrm{GL}_{r}$. The general case was considered in [4]. For a local $G$-shtuka $\mathcal{G}$ over $S$ there exists an étale covering $S^{\prime} \rightarrow S$ and a trivialisation $\underline{\mathcal{G}} \times{ }_{S} S^{\prime} \cong\left(\left(L^{+} G\right)_{S^{\prime}}, b \sigma^{*}\right)$ with $b \in L G\left(S^{\prime}\right)$; see [56, Proposition 2.2(c)] and [4, Proposition 2.4].

Note that we may view $\operatorname{Spf} \mathbb{F}_{q} \llbracket \zeta \rrbracket$ as an ind-scheme. By $\mathcal{F} \ell_{G}$ we denote the affine flag variety of $G$ over $\mathbb{F}_{q}$; compare Section 2 . We may form the fibre product $\widehat{\mathcal{F}}_{G}:=$ $\mathcal{F} \ell_{G} \widehat{\times}_{\mathbb{F}_{q}} \operatorname{Spf} \mathbb{F}_{q} \llbracket \zeta \rrbracket$ in the category of ind-schemes. By [4, Theorem 4.4] it represents the functor on $\mathcal{N} i l p_{\mathbb{F}_{q} \llbracket \zeta \rrbracket}$ with

$$
\begin{aligned}
\widehat{\mathcal{F}}_{G}(S)=\{ & \text { Isomorphism classes of pairs }(\mathcal{G}, \delta) \text { where } \mathcal{G} \text { is an } L^{+} G \text {-torsor on } S \text { and } \\
& \left.\delta: L \mathcal{G} \stackrel{\sim}{\longrightarrow} L G_{S} \text { is an isomorphism of the associated } L G \text {-torsors }\right\} .
\end{aligned}
$$

We consider local $G$-shtukas that satisfy an additional boundedness condition. Similar to $[4, \S 4.2]$, we introduce the notion of a bound $\hat{Z}$ and its reflex $\operatorname{ring} R_{\hat{Z}}$, which is a finite extension of $\mathbb{F}_{q} \llbracket \zeta \rrbracket$. Our bounds are defined as closed ind-subschemes $\hat{Z} \subset \widehat{\mathcal{F}} \ell_{G, R}=$ $\mathcal{F} \ell_{G} \widehat{\times}_{\mathbb{F}_{q}}$ Spf $R$ satisfying certain additional properties. Here $R$ is a finite extension of $\mathbb{F}_{q} \llbracket \zeta \rrbracket$. In particular, we allow more general bounds than usual, in the sense that they do not have to correspond directly to some coweight $\mu$ (which in the classical context even had to be minuscule). We consider local $G$-shtukas $\underline{\mathcal{G}}=\left(\mathcal{G}, \tau_{\mathcal{G}}\right)$ over schemes in $\mathcal{N} i l p_{R_{\hat{Z}}}$ such that the singularities of the morphism $\tau_{\mathcal{G}}^{-1}$ are bounded by $\hat{Z}$; compare Definition 2.2. In this case we say that $\underline{\mathcal{G}}$ is bounded by $\hat{Z}^{-1}$; see Remark 2.3 (a) for a comment on this terminology. These bounded local $G$-shtukas can be seen as the function field analogues of $p$-divisible groups with extra structure. We write $R_{\hat{Z}}=\kappa \llbracket \xi \rrbracket$, let $E:=E_{\hat{Z}}:=\kappa((\xi))$ be its fraction field and let $\breve{R}_{\hat{Z}}=\mathbb{F} \llbracket \xi \rrbracket$ and $\breve{E}:=\breve{E}_{\hat{Z}}:=\mathbb{F}((\xi))$ be the completions of the maximal unramified extensions.

One can then consider the usual Rapoport-Zink type moduli space representing the following functor: Let $\underline{\mathbb{G}}_{0}$ be a local $G$-shtuka over $\mathbb{F}$ and consider the functor 
$\breve{\mathcal{M}}_{\underline{\mathbb{G}}_{0}}^{\hat{Z}^{-1}}:\left(\mathcal{N} i l p_{\breve{R}_{\hat{Z}}}\right)^{o} \longrightarrow \mathcal{S} e t s$

$S \longmapsto\{$ Isomorphism classes of $(\underline{\mathcal{G}}, \bar{\delta})$ : where $\underline{\mathcal{G}}$ is a local $G$-shtuka over $S$ bounded by $\hat{Z}^{-1}$ and $\bar{\delta}: \underline{\mathcal{G}}_{\bar{S}} \rightarrow \underline{\mathbb{G}}_{0, \bar{S}}$ is a quasi-isogeny over $\left.\bar{S}\right\}$.

Here $\bar{S}:=\mathrm{V}_{S}(\zeta)$ is the zero locus of $\zeta$ in $S$. The functor $\breve{\mathcal{M}}_{\mathbb{G}_{0}}^{\hat{Z}^{-1}}$ is ind-representable by a formal scheme over $\operatorname{Spf} \breve{R}_{\hat{Z}}$ that is locally formally of finite type and separated; see [4, Theorem 4.18]. The group $J=\operatorname{QIsog}_{\mathbb{F}}\left(\underline{\mathbb{G}}_{0}\right)$ of self-quasi-isogenies of $\underline{\mathbb{G}}_{0}$ acts on $\breve{\mathcal{M}}_{\mathbb{G}_{0}}^{\hat{Z}^{-1}}$ via $g:(\underline{\mathcal{G}}, \bar{\delta}) \mapsto(\underline{\mathcal{G}}, g \circ \bar{\delta})$ for $g \in \mathrm{QIsog}_{\mathbb{F}}\left(\underline{\mathbb{G}}_{0}\right)$.

We consider the generic fibre $\left(\breve{\mathcal{M}}_{\mathbb{G}_{0}}^{\hat{Z}^{-1}}\right)$ an of this moduli space as a strictly $\breve{E}$-analytic space in the sense of Berkovich [7], [8]. Using the fully faithful functors [8, §1.6] and [63, (1.1.11)] from strictly $\breve{E}$-analytic spaces to rigid analytic spaces over $\breve{E}$, respectively from rigid analytic spaces to Huber's analytic adic spaces, many of the results below can be formulated likewise in terms of rigid analytic, respectively analytic adic spaces. However, because we want to use étale fundamental groups and local systems on these spaces, we prefer in this work the Berkovich point of view for which such a theory exists in the literature.

As in [4, Definition 3.5], we consider the rational (dual) Tate module of the universal local $G$-shtuka over each connected component $Y$ of $\left(\breve{\mathcal{M}}_{\mathbb{\mathbb { G }}_{0}}^{\hat{Z}^{-1}}\right)^{\text {an }}$; see Section 7 . It is a tensor functor

$$
\check{V}_{\underline{\mathcal{G}}, \bullet}: \operatorname{Rep}_{\mathbb{F}_{q}((z))} G \rightarrow \operatorname{Rep}_{\mathbb{F}_{q}((z))}^{\text {cont }}\left(\pi_{1}^{\text {ét }}(Y, \bar{s})\right) .
$$

Here $\operatorname{Rep}_{\mathbb{F}_{q}((z))} G$ denotes the Tannakian category of $\mathbb{F}_{q}((z))$-rational representations of $G$, and $\operatorname{Rep}_{\mathbb{F}_{q}((z))}^{\text {cont }}\left(\pi_{1}^{\text {ét }}(Y, \bar{s})\right)$ denotes the category of finite-dimensional $\mathbb{F}_{q}((z))$-vector spaces with a continuous action of de Jong's [31, §2] étale fundamental group $\pi_{1}^{\text {ét }}(Y, \bar{s})$, where $\bar{s}$ is a fixed base point in the given component.

Trivialising the rational Tate module up to the action of $K$ for each compact open subgroup $K \subset G\left(\mathbb{F}_{q}((z))\right)$, we obtain a tower $\left(\breve{\mathcal{M}}^{K}\right)_{K}$ of analytic spaces. Each of the spaces is equipped with an action of the group $J=\operatorname{QIsog}_{\mathbb{F}}\left(\mathbb{\mathbb { G }}_{0}\right)$ that is induced by the action on the moduli space $\breve{\mathcal{M}}_{\mathbb{\mathbb { G }}_{0}}^{\hat{Z}^{-1}}$ itself. Furthermore, the group $G\left(\mathbb{F}_{q}((z))\right)$ acts vertically on the tower via Hecke operators; that is, for $g \in G\left(\mathbb{F}_{q}((z))\right)$ we have compatible isomorphisms $g: \breve{\mathcal{M}}^{K} \stackrel{\sim}{\longrightarrow} \breve{\mathcal{M}}^{-1} \mathrm{Kg}$.

In the last section we consider the $\ell$-adic cohomology with compact support of the spaces $\breve{\mathcal{M}}^{K}$ and their limit over $K$ together with induced actions of $J$, of $G\left(\mathbb{F}_{q}((z))\right)$ and of the Weil group $W_{E}$. We provide basic finiteness properties of these cohomology groups and representations. Note that Tate modules, towers of moduli spaces of local $G$-shtukas and their cohomology are also considered in a similar but slightly different context by Neupert in [76]. There, the relation to moduli spaces of global $G$-shtukas and their cohomology is studied.

Besides this construction of the tower of moduli spaces, our second main topic is the definition of the associated period space and the properties of the period morphism. Period 
spaces are strictly $\mathbb{F}_{q}((\zeta))$-analytic spaces in the sense of Berkovich [7], [8]. Because we allow more general bounds than those associated with minuscule coweights, these period spaces have to be defined as subspaces of an affine Grassmannian instead of a (classical) flag variety. To define them, we consider the group scheme $G \times_{\mathbb{F}_{q} \llbracket z \rrbracket} \operatorname{Spec} \mathbb{F}_{q}((\zeta)) \llbracket z-\zeta \rrbracket$ under the homomorphism $\mathbb{F}_{q} \llbracket z \rrbracket \rightarrow \mathbb{F}_{q}((\zeta)) \llbracket z-\zeta \rrbracket, z \mapsto z=\zeta+(z-\zeta)$. Note that because this induces an inclusion $\mathbb{F}_{q}((z)) \rightarrow \mathbb{F}_{q}((\zeta)) \llbracket z-\zeta \rrbracket$, this group is reductive. The associated affine Grassmannian $\operatorname{Gr}_{G}^{\mathbf{B}_{\mathrm{dR}}}$ is the sheaf of sets for the fpqc topology on $\operatorname{Spec} \mathbb{F}_{q}((\zeta))$ associated with the presheaf

$$
X \longmapsto G\left(\mathcal{O}_{X}((z-\zeta))\right) / G\left(\mathcal{O}_{X} \llbracket z-\zeta \rrbracket\right) .
$$

$\operatorname{Gr}_{G}^{\mathbf{B}_{\mathrm{dR}}}$ is an ind-scheme over $\operatorname{Spec} \mathbb{F}_{q}((\zeta))$ which is ind-projective by [77, Theorem 1.4] and [84, Theorem A]. Here, the notation $\mathbf{B}_{\mathrm{dR}}$ refers to the fact that if $C$ is the completion of an algebraic closure of $\mathbb{F}_{q}((\zeta))$, then $C((z-\zeta))$ is the function field analogue of Fontaine's $p$-adic period field $\mathbf{B}_{\mathrm{dR}}$; compare $[49, \S 2.9]$.

For our fixed bound $\hat{Z}$ we call the associated $E$-analytic space $\mathcal{H}_{G, \hat{Z}}^{\text {an }}:=\hat{Z}^{\text {an }}$ the space of Hodge-Pink G-structures bounded by $\hat{Z}$. It is the $E$-analytic space associated with a projective variety $\mathcal{H}_{G, \hat{Z}}$ over $E=E_{\hat{Z}}$ by Proposition 2.6(d) and is a closed subscheme of $\operatorname{Gr}_{G}^{\mathbf{B}_{\mathrm{dR}}} \otimes_{\mathbb{F}_{q}((\zeta))} E$. Let $\underline{\mathbb{G}}_{0}$ be the local $G$-shtuka over $\mathbb{F}$ from above and fix a trivialisation $\underline{\mathbb{G}}_{0} \cong\left(L^{+} G_{\mathbb{F}}, b \sigma^{*}\right)$, where $b \in L G(\mathbb{F})$ represents the Frobenius morphism. The period space $\breve{\mathcal{H}}_{G, \hat{Z}, b}^{w a}$ is then defined as the set of all $\gamma \in \breve{\mathcal{H}}_{G, \hat{Z}}^{\text {an }}:=\mathcal{H}_{G, \hat{Z}}^{\text {an }} \widehat{\otimes}_{E} \breve{E}$ such that $(b, \gamma)$ is weakly admissible. For the usual condition of weak admissibility (checked on all representations of $G$ ) we refer to Definition 4.3. Likewise, one defines the admissible locus $\breve{\mathcal{H}}_{G, \hat{Z}, b}^{a}$ in $\breve{\mathcal{H}}_{G, \hat{Z}}^{\text {an }}$ as the subset over which the universal $\sigma$-bundle has slope zero. In Theorem 4.20 we show that $\breve{\mathcal{H}}_{G, \hat{Z}, b}^{w a}$ and $\breve{\mathcal{H}}_{G, \hat{Z}, b}^{a}$ are open paracompact strictly $\breve{E}$-analytic subspaces of $\breve{\mathcal{H}}_{G, \hat{Z}}^{\text {an }}$.

We prove that there is an étale period morphism

$$
\breve{\pi}:\left(\breve{\mathcal{M}}_{\underline{\mathbb{G}}_{0}}^{\hat{Z}^{-1}}\right)^{\text {an }} \longrightarrow \breve{\mathcal{H}}_{G, \hat{Z}, b}^{a} .
$$

Very roughly, it is defined as follows: Consider the filtration on the universal local $G$ shtuka on $\left(\mathcal{M}_{\mathbb{G}_{0}}^{\hat{Z}^{-1}}\right)$ an induced by the image of the inverse of the universal Frobenius morphism $\tau_{\underline{\mathcal{G}}^{\text {univ }}}$. This filtration is the function field analogue of the Hodge filtration on the de Rham cohomology and is bounded by $\hat{Z}$. Using the universal quasi-isogeny, one can associate with it a natural filtration on the base change of $\underline{\mathbb{G}}_{0}$, which is bounded by $\hat{Z}$. Strictly speaking, we carry out this construction with the Hodge-Pink G-structure instead of the filtration; see Definition 4.1 and Remark 4.4(a). The reason for this is again that as we allow nonminuscule bounds, the Hodge-Pink $G$-structure contains more information than just the Hodge filtration. The former yields a point of $\breve{\mathcal{H}}_{G, \hat{Z}, b}^{a}$. This period morphism also induces compatible period morphisms for all elements $\breve{\mathcal{M}}^{K}$ of the tower of coverings. In Theorem 8.1(a) we show that the image of the period morphism is equal to a suitable union of connected components of $\breve{\mathcal{H}}_{G, \hat{z}, b}^{a}$.

There is an analogy between the theory of local $G$-shtukas and the theory of $p$-divisible groups $[49, \S 3.9]$. In this sense, our results have natural counterparts in the theory of $p$-divisible groups, for particular cases by [51] and in general by Scholze and Weinstein 
[89, [90] using the Fargues-Fontaine curve [37]. One main difference is that in the function field case, the flag variety $\mathcal{F} \ell_{G}$ is an honest ind-scheme. In addition, the Fargues-Fontaine curve is replaced by its role model, the Hartl-Pink curve [55]. This allows us to consider nonminuscule Hodge-Pink structures and to work without Scholze's theory of diamonds. One feature of our theory is the group-theoretic approach, which makes the results automatically functorial in the group $G$; see Remarks 3.7, 4.21 and 7.19. Interestingly, the proofs for local $G$-shtukas in this work had to be largely different from the techniques used for $p$-divisible groups and are technically quite involved.

\section{Bounded local $G$-shtukas}

Recall that we fixed a parahoric group scheme $G$ over $\operatorname{Spec}_{q} \llbracket z \rrbracket$.

For an $\mathbb{F}_{q}$-scheme $S$ we let $\mathcal{O}_{S} \llbracket z \rrbracket$ be the sheaf of $\mathcal{O}_{S}$-algebras on $S$ for the fpqc topology whose ring of sections on an $S$-scheme $Y$ is the ring of power series $\mathcal{O}_{S} \llbracket z \rrbracket(Y):=$ $\Gamma\left(Y, \mathcal{O}_{Y}\right) \llbracket z \rrbracket$. This is indeed a sheaf being the countable direct product of $\mathcal{O}_{S}$. A sheaf $M$ of $\mathcal{O}_{S} \llbracket z \rrbracket$-modules on $S$ that is finite free fpqc-locally on $S$ is already finite free Zariski-locally on $S$ by [56, Proposition 2.3]. We call those modules locally free sheaves of $\mathcal{O}_{S} \llbracket z \rrbracket$-modules. Let $\mathcal{O}_{S}((z))$ be the fpqc sheaf of $\mathcal{O}_{S}$-algebras on $S$ associated with the presheaf $Y \mapsto \Gamma\left(Y, \mathcal{O}_{Y}\right) \llbracket z \rrbracket\left[\frac{1}{z}\right]$. If $Y$ is quasi-compact, then $\mathcal{O}_{S}((z))(Y)=\Gamma\left(Y, \mathcal{O}_{Y}\right) \llbracket z \rrbracket\left[\frac{1}{z}\right]$ by $[32$, Tag $009 \mathrm{~F}]$. The group of positive loops associated with $G$ is the infinite-dimensional affine group scheme $L^{+} G$ over $\mathbb{F}_{q}$ whose $S$-valued points are $L^{+} G(S):=G\left(\mathcal{O}_{S} \llbracket z \rrbracket(S)\right)=$ $G\left(\Gamma\left(S, \mathcal{O}_{S}\right) \llbracket z \rrbracket\right)$. The group of loops associated with $G$ is the ind-group-scheme $L G$ over $\mathbb{F}_{q}$ that represents the fpqc sheaf of groups $S \longmapsto L G(S):=G\left(\mathcal{O}_{S}((z))(S)\right)$. A good reference for the theory of ind-schemes is $[6, \S 7.11]$. The affine flag variety $\mathcal{F} \ell_{G}$ associated with $G$ is the fpqc sheaf associated with the presheaf

$$
S \longmapsto L G(S) / L^{+} G(S)=G\left(\mathcal{O}_{S}((z))(S)\right) / G\left(\mathcal{O}_{S} \llbracket z \rrbracket(S)\right)
$$

on the category of $\mathbb{F}_{q}$-schemes. Pappas and Rapoport [77, Theorem 1.4] and Richarz [84, Theorem A] showed that $\mathcal{F} \ell_{G}$ is represented by an ind-scheme that is ind-projective over $\mathbb{F}_{q}$ and that the natural morphism $L G \rightarrow \mathcal{F} \ell_{G}$ admits sections locally for the étale topology. We crucially use the ind-projectivity of $\mathcal{F} \ell_{G}$ in Propositions 2.6 and 7.8. By [77, Theorem 0.1], after base change to $\mathbb{F}$ the connected components of $L G \widehat{\otimes}_{\mathbb{F}_{q}} \mathbb{F}$ and $\mathcal{F} \ell_{G} \widehat{\otimes}_{\mathbb{F}_{q}} \mathbb{F}$ are in canonical bijection to the coinvariants $\pi_{1}(G)_{I}$. Here $\pi_{1}(G)$ is Borovoi's fundamental group [16, Chapter 1], defined as $\pi_{1}(G):=X_{*}(T) /$ (coroot lattice) for a maximal torus $T$ of

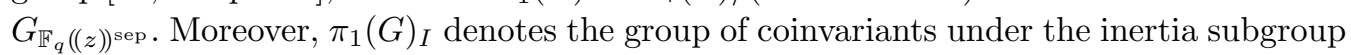
$I$ of $\Gamma=\operatorname{Gal}\left(\mathbb{F}_{q}((z))^{\operatorname{sep}} / \mathbb{F}_{q}((z))\right)$. The bijection $\pi_{0}\left(\mathcal{F} \ell_{G} \widehat{\otimes}_{\mathbb{F}_{q}} \mathbb{F}\right)=\pi_{0}\left(L G \widehat{\otimes}_{\mathbb{F}_{q}} \mathbb{F}\right) \cong \pi_{1}(G)_{I}$ is induced by the Kottwitz homomorphism $\kappa_{G}: L G(\mathbb{F})=G(\mathbb{F}((z))) \rightarrow \pi_{1}(G)_{I}$ (introduced by Kottwitz in [71]; for the reformulation used here, compare [77, 2.a.2]). It induces a bijection between the set $\pi_{0}(L G)=\pi_{0}\left(\mathcal{F} \ell_{G}\right)$ and the set of $\langle\sigma\rangle$-orbits in $\pi_{1}(G)_{I}$ by [76, Lemma 2.2.6], a set that is in general no longer a group.

Remark 2.1. We will define bounds on local $G$-shtukas as (equivalence classes of) certain ind-subschemes of $\widehat{\mathcal{F}}_{G, R}:=\mathcal{F} \ell_{G} \widehat{\times}_{\mathbb{F}_{q}}$ Spf $R$ where $R$ is a finite extension of $\mathbb{F}_{q} \llbracket \zeta \rrbracket$. In order to define and consider also the generic fibre of the associated moduli spaces, one needs 
to bound the singularities with respect to $z-\zeta$ of the local $G$-shtukas. In particular, our definition is more restrictive than the one in [4, Definitions 4.5 and 4.8]. To encode this condition in our notion of bounds, we have to compare $\widehat{\mathcal{F}}_{G, R}$ to the following closed ind-subschemes associated with a representation of $G$.

If $A=\mathbb{F}_{q} \llbracket z \rrbracket$ or $A=\mathbb{F}_{q}((z))$, we let $\operatorname{Rep}_{A} G$ denote the category of (algebraic) representations of $G$ on finite free $A$-modules. Here, we consider representations $\rho: G \rightarrow$ $\mathrm{SL}_{r}$ over $\mathbb{F}_{q} \llbracket z \rrbracket$ and the induced functor $\mathcal{G} \mapsto \rho_{*} \mathcal{G}$ from $L^{+} G$-torsors to $L^{+} \mathrm{SL}_{r}$-torsors, which in turn yields a morphism $\rho_{*}: \widehat{\mathcal{F}}_{G} \rightarrow \widehat{\mathcal{F}}_{\mathrm{SL}_{r}}$. Here, $\widehat{\mathcal{F l}}_{G}:=\mathcal{F} \ell_{G, \mathbb{F}_{q} \llbracket \zeta \rrbracket}$. The category of $L^{+} \mathrm{SL}_{r}$-torsors on $S$ is equivalent to the category of pairs $(M, \alpha)$, where $M$ is a finite locally free $\mathcal{O}_{S} \llbracket z \rrbracket$-module of rank $r$ on $S$ and $\alpha: \wedge_{\mathcal{O}_{S} \llbracket z \rrbracket}^{r} M \stackrel{\sim}{\longrightarrow} \mathcal{O}_{S} \llbracket z \rrbracket$ is an isomorphism of $\mathcal{O}_{S} \llbracket z \rrbracket$-modules, with isomorphisms as morphisms. We denote the $\mathcal{O}_{S} \llbracket z \rrbracket$-module associated with an $L^{+} \mathrm{SL}_{r}$-torsor $\mathcal{S}$ by $M(\mathcal{S})$. For example, $M\left(\left(L^{+} \mathrm{SL}_{r}\right)_{S}\right)=\mathcal{O}_{S} \llbracket z \rrbracket^{\oplus r}$. For a positive integer $n$ we consider the closed ind-subscheme of $\widehat{\mathcal{F}}_{\mathrm{SL}_{r}}$ given by

$$
\begin{aligned}
\widehat{\mathcal{F}}_{\mathrm{SL}_{r}}^{(n)}(S): & =\left\{\left(\mathcal{S}, \delta: L \mathcal{S} \stackrel{\sim}{\longrightarrow}\left(L \mathrm{SL}_{r}\right)_{S}\right) \in \widehat{\mathcal{F}}_{\mathrm{SL}_{r}}(S): \text { for all } j=1, \ldots, r\right. \text { we have } \\
& \left.\bigwedge_{\mathcal{O}_{S} \llbracket z \rrbracket}^{j} M(\delta)(M(\mathcal{S})) \subset(z-\zeta)^{n\left(j^{2}-j r\right)} \cdot \bigwedge_{\mathcal{O}_{S} \llbracket z \rrbracket}^{j} M\left(\left(L^{+} \mathrm{SL}_{r}\right)_{S}\right)\right\} .
\end{aligned}
$$

It is a $\zeta$-adic formal scheme over $\operatorname{Spf}_{q} \llbracket \zeta \rrbracket$ by [56, Proposition 5.5]; see Example 2.8 for more explanations. Note that the compatibility with the isomorphism $\alpha: \wedge^{r} M(\mathcal{S}) \stackrel{\sim}{\longrightarrow}$ $\mathcal{O}_{S} \llbracket z \rrbracket$ is equivalent to the assertion that the inclusion of the exterior powers in (2.1) is an equality for $j=r$, because $(\mathcal{S}, \delta) \in \widehat{\mathcal{F}}_{\mathrm{SL}_{r}}(S)$ implies $\wedge^{r} M(\delta)=\alpha$. We then require the bounds to factor through some $\widehat{\mathcal{F}}_{\mathrm{SL}_{r}}^{(n)}$; cf. Condition (b)(iv).

A different way to formulate such a condition would be to use the isomorphism

$$
\lim _{n} \widehat{\mathcal{F l}}_{G} \times{\widehat{\mathcal{F}} \ell_{\mathrm{SL}_{r}}} \widehat{\mathcal{F}}_{\mathrm{SL}_{r}}^{(n)} \cong \operatorname{Gr}\left(G_{X}, X\right) \times_{X} \operatorname{Spec} R
$$

where the right-hand side is the BD-Grassmannian associated with $G$ of [84, Definition 3.3]. Here we use that $G$ extends by [84, Lemma 3.1] to a smooth affine group scheme $G_{X}$ on a smooth connected curve $X$ over $\mathbb{F}_{q}$ on which $\mathbb{F}_{q} \llbracket z \rrbracket$ is identified with the completion of the local ring at a point $x \in X$. The map Spec $R \rightarrow X$ comes from the inclusion $\mathbb{F}_{q} \llbracket z \rrbracket \hookrightarrow R, z \mapsto \zeta$. The isomorphism (2.2) also induces a comparison between specific bounds with global Schubert varieties of [84]; compare Example 2.7.

We now define bounds by requiring minimal conditions needed to obtain the results of this article. In Remark 2.11 we will discuss further conditions that seem reasonable to impose but that we do not need to assume in this article. We will then also describe more explicitly which bounds can arise, and in Examples 2.7 and 2.8 we will give a more specific class of bounds that depend on cocharacters of the generic fibre of $G$.

Definition 2.2. (a) We fix an algebraic closure $\mathbb{F}_{q}((\zeta))^{\text {alg }}$ of $\mathbb{F}_{q}((\zeta))$ and consider pairs $\left(R, \hat{Z}_{R}\right)$, where $R / \mathbb{F}_{q} \llbracket \zeta \rrbracket$ is a finite extension of discrete valuation rings such that $R \subset \mathbb{F}_{q}((\zeta))^{\text {alg }}$ and where $\hat{Z}_{R} \subset \widehat{\mathcal{F}}_{G, R}:=\mathcal{F} \ell_{G} \widehat{\times}_{\mathbb{F}_{q}}$ Spf $R$ is a closed ind-subscheme. Two such pairs $\left(R, \hat{Z}_{R}\right)$ and $\left(R^{\prime}, \hat{Z}_{R^{\prime}}^{\prime}\right)$ are equivalent if for some finite extension of discrete valuation rings $\widetilde{R} / \mathbb{F}_{q} \llbracket \zeta \rrbracket$ with $R, R^{\prime} \subset \widetilde{R}$ the two closed ind-subschemes 
$\hat{Z}_{R} \widehat{\times}_{\operatorname{Spf} R} \operatorname{Spf} \widetilde{R}$ and $\hat{Z}_{R^{\prime}}^{\prime} \widehat{\times}_{\operatorname{Spf} R^{\prime}} \operatorname{Spf} \widetilde{R}$ of $\widehat{\mathcal{F}}_{G, \widetilde{R}}$ are equal. By [4, Remark 4.6], this then holds for all such rings $\widetilde{R}$.

(b) A bound is an equivalence class $\hat{Z}:=\left[\left(R, \hat{Z}_{R}\right)\right]$ of pairs $\left(R, \hat{Z}_{R}\right)$ as above satisfying the following properties:

(i) All $\hat{Z}_{R} \subset \widehat{\mathcal{F}}_{G, R}$ are stable under the left $L^{+} G$-action.

(ii) The special fibre $Z_{R}:=\hat{Z}_{R} \widehat{\times}_{\text {Spf } R}$ Spec $\kappa_{R}$ is a quasi-compact subscheme of $\mathcal{F} \ell_{G} \widehat{\times}_{\mathbb{F}_{q}} \kappa_{R}$ where $\kappa_{R}$ is the residue field of $R$. (By [4, Remark 4.10] this implies that the $\hat{Z}_{R}$ are formal schemes in the sense of $\left[44, \mathrm{I}_{\text {new }}, \S 10\right]$.)

(iii) $\hat{Z}_{R}$ is a $\zeta$-adic formal scheme over $\operatorname{Spf} R$.

(iv) There is a faithful representation $\rho: G \hookrightarrow \mathrm{SL}_{r}$ over $\mathbb{F}_{q} \llbracket z \rrbracket$ and a positive integer $n$ such that all of the induced morphisms $\rho_{*}: \hat{Z}_{R} \rightarrow \widehat{\mathcal{F}}_{\mathrm{SL}_{r}, R}$ factor through $\widehat{\mathcal{F}}_{\mathrm{SL}_{r}, R}^{(n)}$.

(v) Let $\hat{Z}_{R}^{\text {an }}$ be the strictly $R\left[\frac{1}{\zeta}\right]$-analytic space associated with $\hat{Z}_{R}$. By Proposition 2.6(d) there is a closed subscheme $\hat{Z}_{E}$ of the affine Grassmannian $\operatorname{Gr}_{G}^{\mathbf{B}_{\mathrm{dR}}} \times_{\mathbb{F}_{q}((\zeta))} \operatorname{Spec} E_{\hat{Z}}$ from (1.2) such that $\hat{Z}_{R}^{\mathrm{an}}$ arises by base change to $R\left[\frac{1}{\zeta}\right]$ from the strictly $E_{\hat{Z}^{-}}$-analytic space $\left(\hat{Z}_{E}\right)$ an associated with $\hat{Z}_{E}$. Then we require that $\hat{Z}_{E}$, and hence also all of the $\hat{Z}_{R}^{\text {an }}$ are invariant under the left multiplication of $G(\cdot \llbracket z-\zeta \rrbracket)$ on $\mathrm{Gr}_{G}^{\mathbf{B}_{\mathrm{dR}}}$.

(c) The reflex ring $R_{\hat{Z}}$ of a bound $\hat{Z}=\left[\left(R, \hat{Z}_{R}\right)\right]$ is the intersection of the fixed field of $\left\{\gamma \in \operatorname{Aut}_{\mathbb{F}_{q} \llbracket \zeta \rrbracket}\left(\mathbb{F}_{q}((\zeta))^{\text {alg }}\right): \gamma(\hat{Z})=\hat{Z}\right\}$ in $\mathbb{F}_{q}((\zeta))^{\text {alg }}$ with all of the finite extensions $R \subset$ $\mathbb{F}_{q}((\zeta))^{\text {alg }}$ of $\mathbb{F}_{q} \llbracket \zeta \rrbracket$ over which a representative $\hat{Z}_{R}$ of $\hat{Z}$ exists. We write $R_{\hat{Z}}=\kappa \llbracket \xi \rrbracket$ and call its fraction field $E:=E_{\hat{Z}}=\kappa((\xi))$ the reflex field of $\hat{Z}$. We let $\breve{R}_{\hat{Z}}:=\mathbb{F} \llbracket \xi \rrbracket$ and $\breve{E}:=\breve{E}_{\hat{Z}}:=\mathbb{F}((\xi))$ be the completions of their maximal unramified extensions, where $\mathbb{F}$ is an algebraic closure of the finite field $\kappa$.

(d) Let $\hat{Z}=\left[\left(R, \hat{Z}_{R}\right)\right]$ be a bound with reflex $\operatorname{ring} R_{\hat{Z}}$. Let $\mathcal{G}$ and $\mathcal{G}^{\prime}$ be $L^{+} G$-torsors over a scheme $S \in \mathcal{N} i l p_{R_{\hat{Z}}}$ and let $\delta: L \mathcal{G} \stackrel{\sim}{\longrightarrow} L \mathcal{G}^{\prime}$ be an isomorphism of the associated $L G$-torsors. We consider an étale covering $S^{\prime} \rightarrow S$ over which trivialisations $\alpha: \mathcal{G} \stackrel{\sim}{\longrightarrow}$ $\left(L^{+} G\right)_{S^{\prime}}$ and $\alpha^{\prime}: \mathcal{G}^{\prime} \stackrel{\sim}{\longrightarrow}\left(L^{+} G\right)_{S^{\prime}}$ exist. Then the automorphism $\alpha^{\prime} \circ \delta \circ \alpha^{-1}$ of $(L G)_{S^{\prime}}$ corresponds to a morphism $S^{\prime} \rightarrow L G \widehat{\times}_{\mathbb{F}_{q}} \operatorname{Spf} R_{\hat{Z}}$. We say that $\delta$ is bounded by $\hat{Z}$ if for every such trivialisation and for every finite extension $R$ of $\mathbb{F}_{q} \llbracket \zeta \rrbracket$ over which a representative $\hat{Z}_{R}$ of $\hat{Z}$ exists the induced morphism

$$
S^{\prime} \widehat{\times}_{R_{\hat{Z}}} \operatorname{Spf} R \longrightarrow L G \widehat{\times}_{\mathbb{F}_{q}} \operatorname{Spf} R \longrightarrow \widehat{\mathcal{F}}_{G, R}
$$

factors through $\hat{Z}_{R}$. Furthermore, we say that a local $G$-shtuka $\mathcal{G}=\left(\mathcal{G}, \tau_{\mathcal{G}}\right)$ is bounded by $\hat{Z}$ if $\tau_{\mathcal{G}}$ is bounded by $\hat{Z}$ and, even more important, that $\underline{\mathcal{G}}$ is bounded by $\hat{Z}^{-1}$ if the inverse $\tau_{\mathcal{G}}^{-1}$ of its Frobenius is bounded by $\hat{Z}$; compare the remark below.

Let us explain the conditions of this definition in more detail. 
Remark 2.3. (a) The definition of a bound in Definition 2.2(b) is more restrictive than the one in [4, Definition 4.8] where only conditions (b)(i) and (b)(ii) were required. The reason is that in [4] the content of Proposition 2.6 was not needed and the $R\left[\frac{1}{\zeta}\right]$-analytic spaces $\left(\hat{Z}_{R}\right)^{\text {an }}$ were not considered.

In this article we will mainly consider local $G$-shtukas that are bounded by $\hat{Z}^{-1}$. This definition coincides with the notion of boundedness from [4, Definition 4.8(b)] in the following way. If $\hat{Z}$ is a bound in the sense of [4, Definition 4.8], like, for example, our bound $\hat{Z}$, then by Lemma 2.12 there is a bound $\hat{Z}^{-1}$ in the sense of [4, Definition 4.8] and $\tau_{\mathcal{G}}^{-1}$ is bounded by $\hat{Z}$ if and only if $\tau_{\mathcal{G}}$ is bounded by $\hat{Z}^{-1}$.

(b) The reflex ring in Definition 2.2(c) is always the ring of integers of a finite extension of $\mathbb{F}_{q}((\zeta))$. For a detailed explanation of the definition of the reflex ring and a comparison with the number field case, see [4, Remark 4.7]. We do not know whether in general $\hat{Z}$ has a representative over the reflex ring. In contrast, the equivalence class of the $Z_{R}:=\hat{Z}_{R} \widehat{\times}_{\operatorname{Spf} R} \operatorname{Spec} \kappa_{R}$ always has a representative $Z \subset \mathcal{F} \ell_{G} \widehat{\times}_{\mathbb{F}_{q}}$ Spec $\kappa$ over the residue field $\kappa$ of the reflex ring $R_{\hat{Z}}$, because the Galois descent for closed ind-subschemes of $\mathcal{F} \ell_{G}$ is effective. We call $Z$ the special fibre of $\hat{Z}$. It is a projective scheme over $\kappa$ by [56, Lemma 5.4] because $\mathcal{F} \ell_{G}$ is ind-projective.

(c) The condition of Definition 2.2(d) is satisfied for all trivialisations $\alpha$ and $\alpha^{\prime}$ and for all such finite extensions $R$ of $\mathbb{F}_{q} \llbracket \zeta \rrbracket$ if and only if it is satisfied for one trivialisation and for one such finite extension. Indeed, by the $L^{+} G$-invariance of $\hat{Z}$, the definition is independent of the trivialisations. That one finite extension suffices follows from [4, Remark 4.6].

(d) At first glance one might think that conditions (b)(i) and (b)(v) of Definition 2.2 are related. However, in Example 2.9 we show that we really need to impose both of them.

Before we discuss properties of bounds, we recall the following well-known lemma.

Lemma 2.4. Let $f: X \rightarrow Y$ be a morphism of locally Noetherian adic formal schemes. Then $f$ is a closed immersion in the sense of [44, $\mathrm{I}_{\text {new }}$, Definition 10.14.2] if and only if $f$ is adic and an ind-closed immersion of ind-schemes.

Proof. By definition $f$ is a closed immersion if and only if there is a covering of $Y$ by open affine formal subschemes $\operatorname{Spf} B$ such that $X \times_{Y} \operatorname{Spf} B \cong \operatorname{Spf} B / \mathfrak{a}$ for an ideal $\mathfrak{a} \subset B$. In particular, if $I \subset B$ is a finitely generated ideal of definition of $\operatorname{Spf} B$, then $I \cdot B / \mathfrak{a}$ is an ideal of definition of $\operatorname{Spf} B / \mathfrak{a}$ and so $f$ is adic. Moreover, $\operatorname{Spf} B=\lim \operatorname{Spec} B / I^{n}$ and $\operatorname{Spf} B / \mathfrak{a}=\lim \operatorname{Spec} B /\left(\mathfrak{a}+I^{n}\right)$ and so $f$ is an ind-closed immersion of ind-schemes.

To prove the converse, let $\mathcal{I} \subset \mathcal{O}_{Y}$ be an ideal sheaf of definition. Because $f$ is adic, $\mathcal{I} \cdot \mathcal{O}_{X}$ is an ideal sheaf of definition of $X$. That $f$ is an ind-closed immersion means that $X_{n}:=\left(X, \mathcal{O}_{X} / \mathcal{I}^{n}\right) \hookrightarrow Y_{n}:=\left(Y, \mathcal{O}_{Y} / \mathcal{I}^{n}\right)$ is a closed immersion of schemes. So there is a sheaf of ideals $\mathfrak{a}_{n} \subset \mathcal{O}_{Y} / \mathcal{I}^{n}$ defining $X_{n}$. Moreover, $\mathfrak{a}_{n}=\mathfrak{a}_{n+1} \cdot \mathcal{O}_{Y} / \mathcal{I}^{n}$, because $X_{n}=X_{n+1} \times_{Y_{n+1}} Y_{n}$. Let $\mathfrak{a}:=\operatorname{lima}_{n} \subset \lim _{\longleftarrow} \mathcal{O}_{Y} / \mathcal{I}^{n}=\mathcal{O}_{Y}$. Because $Y$ is locally Noetherian, $\mathfrak{a}$ is a coherent sheaf of $\mathcal{O}_{Y \text {-modules by }}$ [44, $\mathrm{I}_{\text {new }}$, Theorem 10.10.2]. Then 
$X_{n}=\left(X,\left.\left(\mathcal{O}_{Y} /\left(\mathfrak{a}+\mathcal{I}^{n}\right)\right)\right|_{X}\right)$ and $X=\left(X,\left.\left(\mathcal{O}_{Y} / \mathfrak{a}\right)\right|_{X}\right)$. This proves that $f$ is a closed immersion in the sense of [44, $\mathrm{I}_{\text {new }}$, Definition 10.14.2].

Remark 2.5. Without the assumption that $f$ is adic, the conclusion of the lemma is false, as the following example shows. Let $Y=\lim _{\longrightarrow} Y_{n}$ with $Y_{n}=\operatorname{Spec} \mathbb{F}_{q} \llbracket \zeta \rrbracket[x] /\left(\zeta^{n}\right)$ and $X=\lim _{\longrightarrow} X_{n}$ with $X_{n}=\operatorname{Spec} \mathbb{F}_{q} \llbracket \zeta \rrbracket[x] /(\zeta, x)^{n}$. Then $X=\operatorname{Spf} \mathbb{F}_{q} \llbracket \zeta, x \rrbracket \rightarrow Y=\operatorname{Spf} \mathbb{F}_{q} \llbracket \zeta \rrbracket\langle x\rangle$, with the notation of (6.3), is an ind-closed immersion of ind-schemes but not a closed immersion of formal schemes.

In the next proposition we associate with a bound $\hat{Z}$ a strictly $\mathbb{F}_{q}((\zeta))$-analytic space in the sense of Berkovich [7], [8]. On the category of $\mathbb{F}_{q}((\zeta))$-analytic spaces we consider the étale topology; see $[8, \S 4.1]$.

Proposition 2.6. Let $\hat{Z}=\left[\left(R, \hat{Z}_{R}\right)\right]$ be a bound with reflex ring $R_{\hat{Z}}$, and let $E:=E_{\hat{Z}}:=$ $R_{\hat{Z}}\left[\frac{1}{\zeta}\right]$ be its field of fractions. We only assume that $Z$ satisfies conditions $(b)(i)-(b)(i v)$ from Definition 2.2 but not condition $(b)(v)$, whose formulation uses the results of the present proposition.

(a) Then for every representation $\rho: G \rightarrow \mathrm{SL}_{r}$ over $\mathbb{F}_{q} \llbracket z \rrbracket$ there is a positive integer $n$ such that all of the induced morphisms $\rho_{*}: \hat{Z}_{R} \hookrightarrow \widehat{\mathcal{F}} \ell_{G, R} \rightarrow \widehat{\mathcal{F}}_{\mathrm{SL}_{r}}, R$ factor through $\widehat{\mathcal{F}}_{\mathrm{SL}_{r}, R}^{(n)}$.

(b) $\widehat{\mathcal{F}}_{\mathrm{SL}_{r}}^{(n)}$ is a $\zeta$-adic formal scheme over $\operatorname{Spf} \mathbb{F}_{q} \llbracket \zeta \rrbracket$. It is the $\zeta$-adic completion of a projective scheme over $\operatorname{Spec} \mathbb{F}_{q} \llbracket \zeta \rrbracket$ that we also denote by $\widehat{\mathcal{F l}}_{\mathrm{SL}_{r}}^{(n)}$. The corresponding strictly $\mathbb{F}_{q}((\zeta))$-analytic space $\left(\widehat{\mathcal{F}}_{\mathrm{SL}_{r}}^{(n)}\right)^{\text {an }}$ is the analytification of the projective scheme $\widehat{\mathcal{F}}_{\mathrm{SL}_{r}}^{(n)} \times_{\mathbb{F}_{q} \llbracket \zeta \rrbracket} \operatorname{Spec} \mathbb{F}_{q}((\zeta))$ over $\operatorname{Spec} \mathbb{F}_{q}((\zeta))$ that represents the sheaf of sets for the étale topology associated with the presheaf

$$
\begin{gathered}
X \longmapsto\left\{g \bmod \mathrm{SL}_{r}\left(\mathcal{O}_{X} \llbracket z-\zeta \rrbracket\right) \in \mathrm{SL}_{r}\left(\mathcal{O}_{X}((z-\zeta))\right) / \mathrm{SL}_{r}\left(\mathcal{O}_{X} \llbracket z-\zeta \rrbracket\right):\right. \\
\text { all } \left.j \times j \text {-minors of } g \text { lie in }(z-\zeta)^{n\left(j^{2}-j r\right)} \mathcal{O}_{X} \llbracket z-\zeta \rrbracket \text { for all } j\right\} .
\end{gathered}
$$

The scheme $\widehat{\mathcal{F}}_{\mathrm{SL}_{r}}^{(n)} \times_{\mathbb{F}_{q} \llbracket \zeta \rrbracket} \operatorname{Spec} \mathbb{F}_{q}((\zeta))$ is a closed subscheme of the affine Grassmannian $\mathrm{Gr}_{\mathrm{SL}_{r}}^{\mathbf{B}_{d R}}$ from (1.2).

(c) If $n$ and $\rho$ are as in (a) such that $\rho$ is faithful with quasi-affine quotient $\mathrm{SL}_{r} / G$, then all $\rho_{*}: \hat{Z}_{R} \hookrightarrow \widehat{\mathcal{F}}_{\mathrm{SL}_{r}, R}^{(n)}$ are closed immersions of formal schemes over $\operatorname{Spf} R$ in the sense of [44, $\mathrm{I}_{\mathrm{new}}$, Definition 10.14.2].

(d) All $\hat{Z}_{R}$ are $\zeta$-adic formal schemes, projective over Spf $R$. All of their associated $R\left[\frac{1}{\zeta}\right]$-analytic spaces $\left(\hat{Z}_{R}\right)^{\text {an }}$ arise by base change to $R\left[\frac{1}{\zeta}\right]$ from one strictly $E_{\hat{Z}^{-}}$ analytic space $\hat{Z}^{a n}:=\left(\hat{Z}_{E}\right)^{\text {an }}$ associated with a projective scheme $\hat{Z}_{E}$ over $\operatorname{Spec} E_{\hat{Z}}$. The latter is a closed subscheme of the affine Grassmannian $\operatorname{Gr}_{G}^{\mathbf{B}_{d R}} \times_{\mathbb{F}_{q}((\zeta))} \operatorname{Spec} E_{\hat{Z}}$ from (1.2). 
Remark. The proof of statements (c) and (d) uses the ind-projectivity of the affine flag variety $\mathcal{F} \ell_{G}$. If $G$ is not parahoric, it is not clear to us whether $\rho_{*}: \hat{Z}_{R} \hookrightarrow \widehat{\mathcal{F}}_{\mathrm{SL}_{r}, R}^{(n)}$ is a locally closed immersion of $\zeta$-adic formal schemes.

Proof of Proposition 2.6. (a) Let $\rho^{\prime}: G \hookrightarrow \mathrm{SL}_{r^{\prime}}$ and $n^{\prime}$ be the representation and the integer from Definition 2.2(b) for which all $\rho_{*}^{\prime}: \hat{Z}_{R} \rightarrow \widehat{\mathcal{F}}_{\mathrm{SL}_{r^{\prime}}, R}$ factor through $\widehat{\mathcal{F}}_{\mathrm{SL}_{r^{\prime}}, R}^{\left(n^{\prime}\right)}$.

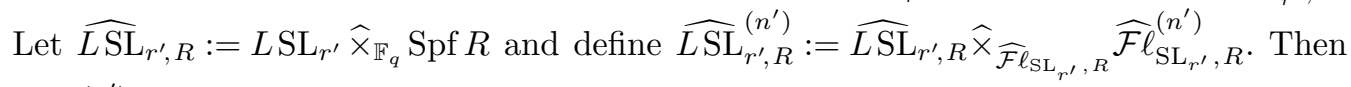
$\widehat{L S L}_{r^{\prime}, R}^{\left(n^{\prime}\right)}(S)=$

$$
\left\{g \in \widehat{L S L}_{r^{\prime}, R}(S) \text { : all } j \times j \text {-minors of } g \text { lie in }(z-\zeta)^{n^{\prime}\left(j^{2}-j r^{\prime}\right)} \mathcal{O}_{S}(S) \llbracket z \rrbracket \forall j=1, \ldots, r^{\prime}\right\} \text {. }
$$

This implies that $\widehat{L S L}_{r^{\prime}, R}^{\left(n^{\prime}\right)}$ is an infinite-dimensional affine formal scheme over Spf $R$. Thus, its closed subscheme $\widehat{L G}_{R}^{\left(n^{\prime}\right)}:=\left(L G \widehat{\times}_{\mathbb{F}_{q}} \operatorname{Spf} R\right) \widehat{\times}_{\widehat{L S L}_{r^{\prime}, R}} \widehat{L S L}_{r^{\prime}, R}^{\left(n^{\prime}\right)}$ is also affine. By [4, Remark 4.10], the ind-schemes $\hat{Z}_{R}$ are in fact formal schemes over $\operatorname{Spf} R$ in the sense of $\left[44, \mathrm{I}_{\text {new }}\right]$. Because the morphism $L G \rightarrow \mathcal{F} \ell_{G}$ has sections étale locally, there is an étale covering of formal schemes $\hat{Z}_{R}^{\prime} \rightarrow \hat{Z}_{R}$ such that the morphism $\hat{Z}_{R}^{\prime} \rightarrow \widehat{\mathcal{F l}}_{G, R}$ factors through $\widehat{L G}_{R}^{\left(n^{\prime}\right)}$. Let $\operatorname{Spf} A \subset \hat{Z}_{R}^{\prime}$ be an affine open formal subscheme with $\operatorname{Spf} A=\underset{\longrightarrow}{\longrightarrow} \operatorname{Spec} A_{i}$ for some $A_{i}$. The induced compatible collection of morphisms $\operatorname{Spec} A_{i} \rightarrow \widehat{L G}_{R}^{\left(n^{\prime}\right)} \hookrightarrow$ $\widehat{L S L}_{r^{\prime}, R}^{\left(n^{\prime}\right)}$ corresponds to a compatible collection of ring homomorphisms $\mathcal{O}\left(\widehat{L S L}_{r^{\prime}, R}^{\left(n^{\prime}\right)}\right) \rightarrow$ $\mathcal{O}\left(\widehat{L G}_{R}^{\left(n^{\prime}\right)}\right) \rightarrow A_{i}$ and thus to a homomorphism $\mathcal{O}\left(\widehat{L S L}_{r^{\prime}, R}^{\left(n^{\prime}\right)}\right) \rightarrow \mathcal{O}\left(\widehat{L G}_{R}^{\left(n^{\prime}\right)}\right) \rightarrow A$. We view the latter as an element $b \in \mathrm{SL}_{r^{\prime}}\left(A \llbracket z \rrbracket\left[\frac{1}{z-\zeta}\right]\right) \cap\left((z-\zeta)^{-n^{\prime}\left(r^{\prime}-1\right)} A \llbracket z \rrbracket\right)^{r^{\prime} \times r^{\prime}}$. It actually lies in $G\left(A \llbracket z \rrbracket\left[\frac{1}{z-\zeta}\right]\right)$, because the closed ind-subscheme $L G \hookrightarrow L \mathrm{SL}_{r^{\prime}}$ is defined by the equations that, applied to the entries of a matrix in $\mathrm{SL}_{r^{\prime}}$, cut out the closed subgroup $\rho^{\prime}: G \hookrightarrow \mathrm{SL}_{r^{\prime}}$.

If now $\rho: G \rightarrow \mathrm{SL}_{r}$ is any representation over $\mathbb{F}_{q} \llbracket z \rrbracket$, then we claim that there is a positive integer $n$ that only depends on $\rho$ and $n^{\prime}$ such that $\rho(b) \in \mathrm{SL}_{r}\left(A \llbracket z \rrbracket\left[\frac{1}{z-\zeta}\right]\right)$ and all $j \times j$ minors of $\rho(b)$ lie in $(z-\zeta)^{n\left(j^{2}-j r\right)} A \llbracket z \rrbracket$ for all $j$. Indeed, equality for $j=r$ always holds because the image of $\rho$ is in $\mathrm{SL}_{r}$. For the other $j$ we realise $\rho$ as a subquotient of $\bigoplus_{i=1}^{i_{0}}\left(\rho^{\prime}\right)^{\otimes l_{i}} \otimes\left(\rho^{\prime \vee}\right)^{\otimes m_{i}}$ for suitable $i_{0}, l_{i}$ and $m_{i}$. Then it is enough to show the claim for this direct sum. Here we can bound all minors by bounds only depending on $n^{\prime}, i_{0}$ and the $l_{i}$ and $m_{i}$. The claim follows. Thus, $\rho_{*}: \hat{Z}_{R} \rightarrow \widehat{\mathcal{F}}_{\mathrm{SL}_{r}, R}$ factors through $\widehat{\mathcal{F}}_{\mathrm{SL}_{r}, R}^{(n)}$

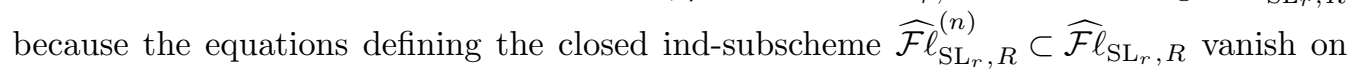
the étale covering $\hat{Z}_{R}^{\prime} \rightarrow \hat{Z}_{R}$.

(b) To show that $\widehat{\mathcal{F}}_{\mathrm{SL}_{r}}^{(n)}(S)$ is projective over $\operatorname{Spf} \mathbb{F}_{q} \llbracket \zeta \rrbracket$, we use the equivalence between $L^{+} \mathrm{SL}_{r}$-torsors over $S$ and pairs $(M, \alpha)$ where $M$ is a locally free $\mathcal{O}_{S} \llbracket z \rrbracket$-module on $S$ and $\alpha: \wedge^{r} M \stackrel{\sim}{\longrightarrow} \mathcal{O}_{S} \llbracket z \rrbracket$ is an isomorphism of $\mathcal{O}_{S} \llbracket z \rrbracket$-modules. Under this equivalence and 
using that $\mathcal{O}_{S} \llbracket z \rrbracket=\mathcal{O}_{S} \llbracket z-\zeta \rrbracket$ for all $S \in \mathcal{N} i l p_{\mathbb{F}_{q} \llbracket \zeta \rrbracket}$, we may identify $\widehat{\mathcal{F}}_{\mathrm{SL}_{r}}^{(n)}(S)$ with the set

$\left\{\right.$ locally free $\mathcal{O}_{S} \llbracket z-\zeta \rrbracket$-submodules $M \subset(z-\zeta)^{-n(r-1)} \mathcal{O}_{S} \llbracket z-\zeta \rrbracket^{\oplus r}$ such that for all $j=1, \ldots, r$ we have the inclusion $\bigwedge_{\mathcal{O}_{S} \llbracket z-\zeta \rrbracket}^{j} M \subset(z-\zeta)^{n\left(j^{2}-j r\right)} \cdot \bigwedge_{\mathcal{O}_{S} \llbracket z-\zeta \rrbracket}^{j} \mathcal{O}_{S} \llbracket z-\zeta \rrbracket^{\oplus r}$ with equality for $j=r\}$.

Note that the quotient $(z-\zeta)^{-n(r-1)} \mathcal{O}_{S} \llbracket z-\zeta \rrbracket^{\oplus r} / M$ is finite locally free as $\mathcal{O}_{S}$-module by [56, Lemma 4.3]. From Cramer's rule (e.g., [22, III.8.6, Formulas (21) and (22)]), one sees that the above condition for $j=r-1$ implies that $M \supset(z-\zeta)^{n(r-1)} \mathcal{O}_{S} \llbracket z-\zeta \rrbracket^{\oplus r}$. By considering the image $\bar{M}$ of $(z-\zeta)^{n(r-1)} M$ in $\left(\mathcal{O}_{S} \llbracket z-\zeta \rrbracket /(z-\zeta)^{2 n(r-1)}\right)^{\oplus r}$ and using arguments similar to [52, Lemma 2.7] (see also [87, Proposition 2.4.6]), we obtain a closed embedding of $\widehat{\mathcal{F}}_{\mathrm{SL}_{r}}^{(n)}$ into the formal $\zeta$-adic completion

$$
\left(\operatorname{Quot}_{\mathcal{O}^{r}\left|\operatorname{Spec} \mathbb{F}_{q} \llbracket \zeta \rrbracket[z] /(z-\zeta)^{2 n(r-1)}\right| \operatorname{Spec} \mathbb{F}_{q} \llbracket \zeta \rrbracket}\right) \times_{\operatorname{Spec} \mathbb{F}_{q} \llbracket \zeta \rrbracket} \operatorname{Spf} \mathbb{F}_{q} \llbracket \zeta \rrbracket
$$

of Grothendieck's Quot-scheme whose points over a Spec $\mathbb{F}_{q} \llbracket \zeta \rrbracket$-scheme $S$ are

$$
\begin{aligned}
& \left(\text { Quot }_{\mathcal{O}^{r}\left|\operatorname{Spec} \mathbb{F}_{q} \llbracket \zeta \rrbracket[z] /(z-\zeta)^{2 n(r-1)}\right| \operatorname{Spec}_{q} \llbracket \zeta \rrbracket}\right)(S)= \\
& \left\{\text { finitely presented } \mathcal{O}_{S}[z] /(z-\zeta)^{2 n(r-1)} \text {-submodules } \bar{M} \subset\left(\mathcal{O}_{S}[z] /(z-\zeta)^{2 n(r-1)}\right)^{\oplus r}\right. \\
& \text { whose quotient is finite locally free over } \left.\mathcal{O}_{S}\right\} ;
\end{aligned}
$$

see [43, $\mathrm{n}^{\circ} 221$, Theorem 3.1] or [2, Theorem 2.6]. By the projectivity of the Quot-scheme, $\widehat{\mathcal{F}}_{\mathrm{SL}_{r}}^{(n)}$ is projective over Spf $\mathbb{F}_{q} \llbracket \zeta \rrbracket$. From (2.4) also the description of the sheaf represented by $\left(\widehat{\mathcal{F}}_{\mathrm{SL}_{r}}^{(n)}\right)^{\text {an }}$ follows.

(c) If $\rho: G \hookrightarrow \mathrm{SL}_{r}$ is a faithful representation with quasi-affine quotient $\mathrm{SL}_{r} / \rho(G)$, then Pappas and Rapoport [77, Theorem 1.4] showed that the induced morphism $\rho_{*}: \mathcal{F} \ell_{G} \hookrightarrow$ $\mathcal{F} \ell_{\mathrm{SL}_{r}}$ is a locally ind-closed immersion of ind-schemes. Because $\mathcal{F} \ell_{G}$ is ind-proper by [84, Theorem A], this is even an ind-closed immersion. Because $\hat{Z}_{R}$ is a $\zeta$-adic formal scheme by Definition 2.2(b)(iii), all $\rho_{*}: \hat{Z}_{R} \hookrightarrow \widehat{\mathcal{F}}_{\mathrm{SL}_{r}, R}^{(n)}$ are adic ind-closed immersions of formal schemes over $\operatorname{Spf} R$ and hence closed immersions by Lemma 2.4.

(d) By [77, Proposition 1.3] there is a faithful representation $\rho: G \hookrightarrow \mathrm{SL}_{r}$ as in (a) with quasi-affine quotient $\mathrm{SL}_{r} / \rho(G)$. Therefore, all $\hat{Z}_{R}$ are projective over $\operatorname{Spf} R$ by (b) and (c), and the associated strictly $R\left[\frac{1}{\zeta}\right]$-analytic space $\left(\hat{Z}_{R}\right)^{\text {an }}$ is Zariski-closed in the projective $R\left[\frac{1}{\zeta}\right]$-analytic space $\left(\widehat{\mathcal{F}}_{\mathrm{SL}_{r}, R}^{(n)}\right)^{\text {an }}$. By analytic GAGA [75, Theorem 2.8], $\left(\hat{Z}_{R}\right)^{\text {an }}$ is the analytification of a closed subscheme $\hat{Z}_{R\left[\frac{1}{\zeta}\right]}$ of the projective scheme $\widehat{\mathcal{F}}_{\mathrm{SL}_{r}}^{(n)} \times_{\mathbb{F}_{q} \llbracket \zeta \rrbracket}$ 
$\operatorname{Spec} R\left[\frac{1}{\zeta}\right]$. By [4, Remark 4.7(f)] there is an $R$ over which a representative $\hat{Z}_{R}$ exists, such that $R\left[\frac{1}{\zeta}\right]$ is Galois over $E_{\hat{Z}}$ and $\hat{Z}_{R}$ is invariant under $\operatorname{Gal}\left(R\left[\frac{1}{\zeta}\right] / E_{\hat{Z}}\right)$. Because the Galois descent for projective $E_{\hat{Z}^{\text {-schemes }}}$ is effective by [45, Chapitre VIII, Corollaire 7.7], the scheme $\hat{Z}_{R\left[\frac{1}{\zeta}\right]}$ and its analytification $\left(\hat{Z}_{R}\right)^{\text {an }}$ descend to a projective scheme $\hat{Z}_{E}$ over $\operatorname{Spec} E_{\hat{Z}}$ and its associated strictly $E_{\hat{Z}^{-}}$analytic space $\left(\hat{Z}_{E}\right)^{\text {an }}$.

By our proof of (a), there is an étale covering of $\hat{Z}_{R}$ formed by formal schemes Spf $A$ on which a lift of the inclusion $\hat{Z}_{R} \hookrightarrow \widehat{\mathcal{F}}_{G, R}$ to a point in $G\left(A \llbracket z \rrbracket\left[\frac{1}{z-\zeta}\right]\right)$ exists, which is unique up to multiplication by $G(A \llbracket z \rrbracket)$ on the right. Under the map $A \llbracket z \rrbracket \hookrightarrow$ $A\left[\frac{1}{\zeta}\right] \llbracket z-\zeta \rrbracket, z \mapsto z=\zeta+(z-\zeta)$, this point gives rise to a point in $G\left(A\left[\frac{1}{\zeta}\right]((z-\zeta))\right)$. The latter induces a morphism $\operatorname{Spec} A\left[\frac{1}{\zeta}\right] \rightarrow \operatorname{Gr}_{G}^{\mathbf{B}_{\mathrm{dR}}} \times_{\mathbb{F}_{q}((\zeta))} \operatorname{Spec} R\left[\frac{1}{\zeta}\right]$ and we consider its analytification $(\operatorname{Spf} A)^{\text {an }} \rightarrow\left(\operatorname{Gr}_{G}^{\mathbf{B}_{\mathrm{dR}}} \times_{\mathbb{F}_{q}((\zeta))} \text { Spec } R\left[\frac{1}{\zeta}\right]\right)^{\text {an }}$, which now descends to a morphism $\left(\hat{Z}_{R}\right)^{\text {an }} \rightarrow\left(\operatorname{Gr}_{G}^{\mathbf{B}_{\mathrm{dR}}} \times_{\mathbb{F}_{q}((\zeta))} \operatorname{Spec} R\left[\frac{1}{\zeta}\right]\right)^{\text {an }}$. By Galois descent it descends further to $\left(\hat{Z}_{E}\right)^{\text {an }}$ and provides the closed immersion into $\operatorname{Gr}_{G}^{\mathbf{B}_{\mathrm{dR}}} \times_{\mathbb{F}_{q}((\zeta))} \operatorname{Spec} E_{\hat{Z}}$.

Example 2.7. We describe bounds that arise from a conjugacy class of coweights. Let $E_{0}$ be a finite field extension of $\mathbb{F}_{q}((\zeta))$ and let $\mu: \mathbb{G}_{m, E_{0}} \rightarrow G_{E_{0}}:=G \times_{\mathbb{F}_{q} \llbracket z \rrbracket, z \mapsto \zeta} E_{0}$ be a coweight over $E_{0}$ of the generic fibre of $G$. Because the following construction only depends on the conjugacy class of $\mu$, we may assume that $E_{0}$ is separable over $\mathbb{F}_{q}((\zeta))$ by $\left[14, \S 8.11\right.$, Corollary 1]. Recall the affine Grassmannian $\operatorname{Gr}_{G}^{\mathbf{B}_{\mathrm{dr}}}$ from (1.2). We first define $\hat{Z}_{\preceq \mu, E_{0}}$ as the scheme-theoretic closure in $\operatorname{Gr}_{G, E_{0}}^{\mathbf{B}_{\mathrm{dR}}}:=\operatorname{Gr}_{G}^{\mathbf{B}_{\mathrm{dR}}} \times_{\mathbb{F}_{q}((\zeta))}$ Spec $E_{0}$ of

$$
G\left(E_{0} \llbracket z-\zeta \rrbracket\right) \cdot \mu(z-\zeta) \cdot G\left(E_{0} \llbracket z-\zeta \rrbracket\right) / G\left(E_{0} \llbracket z-\zeta \rrbracket\right) \subset \operatorname{Gr}_{G, E_{0}}^{\mathbf{B}_{\mathrm{dR}}} .
$$

That is, $\hat{Z}_{\preceq \mu, E_{0}}$ is the (reduced) closed Schubert variety associated with $\mu$. Choose a faithful representation $\rho: G \hookrightarrow \mathrm{SL}_{r}$ over $\mathbb{F}_{q} \llbracket z \rrbracket$. Then there is a positive integer $n$ such that the induced morphisms $\rho_{*}: \hat{Z}_{\preceq \mu, E_{0}} \hookrightarrow \mathrm{Gr}_{G, E_{0}}^{\mathbf{B}_{\mathrm{dR}}} \hookrightarrow \mathrm{Gr}_{\mathrm{SL}_{r}, E_{0}}^{\mathbf{B}_{\mathrm{dR}}}$ factors through the closed subscheme $\widehat{\mathcal{F}}_{\mathrm{SL}_{r}, E_{0}}^{(n)}:=\widehat{\mathcal{F}}_{\mathrm{SL}_{r}}^{(n)} \times_{\mathbb{F}_{q} \llbracket \zeta \rrbracket} \operatorname{Spec} E_{0} \subset \operatorname{Gr}_{\mathrm{SL}_{r}, E_{0}}^{\mathbf{B}_{\mathrm{dR}}}$ from (2.3), which is projective over Spec $E_{0}$. We let $R$ be the integral closure of $\mathbb{F}_{q} \llbracket \zeta \rrbracket$ in $E_{0}$ and we let $\hat{Z}_{R}$ be a closed subscheme of the projective $R$-scheme

$$
\widehat{\mathcal{F}}_{G, R} \underset{{\widehat{\mathcal{F}} \ell_{\mathrm{SL}_{r}, R}}^{\times}}{\times}\left(\widehat{\mathcal{F}}_{\mathrm{SL}_{r}}^{(n)} \times_{\mathbb{F}_{q} \llbracket \zeta \rrbracket} \operatorname{Spec} R\right)
$$

with $\hat{Z}_{R} \times{ }_{R} \operatorname{Spec} E_{0}=\hat{Z}_{\preceq \mu, E_{0}}$. For example, one could take $\hat{Z}_{R}$ as the reduced closure of $\hat{Z}_{\preceq \mu, E_{0}}$, which is flat over $\mathbb{F}_{q} \llbracket \zeta \rrbracket$ and which we call $\hat{Z}_{\preceq \mu, R}$. In this case, it coincides with the global Schubert variety of [84, Definition 3.5]; compare Remark 2.1. However, this is not the only possible choice for $\hat{Z}_{R}$.

By our assumption that $G$ is parahoric, $\widehat{\mathcal{F}}_{G, R}$ is ind-projective by [84, Theorem A] and the schemes $(2.5)$ and $\hat{Z}_{R}$ are indeed projective over $\operatorname{Spec} R$. Therefore, the formal $\zeta$-adic completion of $\hat{Z}_{R}$ defines a bound $\hat{Z}$ whose associated strictly $E_{0}$-analytic space $\left(\hat{Z}_{R}\right)^{\text {an }}$ 
arises as the analytification of $\hat{Z}_{E_{0}}$. If $\hat{Z}_{R}=\hat{Z}_{\preceq \mu, R}$, we denote the associated bound by $\hat{Z}_{\preceq \mu}$.

Claim. The reflex field $E_{\hat{Z}_{\preceq \mu}}$ of $\hat{Z}_{\preceq \mu}$ is equal to the reflex field $E_{\mu}$ of the conjugacy class of $\mu$ and is, in particular, separable over $\mathbb{F}_{q}((\zeta))$.

Indeed, the field $E_{\mu}$ is defined as the fixed field in a separable closure $E_{0}$ sep of the group

$$
\left\{\gamma \in \operatorname{Gal}\left(E_{0}{ }^{\text {sep }} / \mathbb{F}_{q}((\zeta))\right): \exists g \in G\left(E_{0}{ }^{\text {sep }}\right) \text { with }{ }^{\gamma} \mu=\operatorname{Int}_{g} \circ \mu\right\}
$$

The field $E_{\mu}$ is contained in $E_{0}$ and, more generally, in every field over which a representative of the conjugacy class of $\mu$ exists, but these inclusions may be strict. To show that $E_{\hat{Z}_{\preceq \mu}} \subset E_{\mu}$, we note that $E_{\hat{Z}_{\preceq \mu}} \subset E_{0} \subset E_{0}$ sep and that every element of the group (2.6) satisfies $\gamma\left(\hat{Z}_{\preceq \mu}\right)=\hat{Z}_{\preceq \mu}$, because $\hat{Z}_{\preceq \mu}$ is defined as the closure of $\hat{Z}_{\preceq \mu, E_{0}}$ and $\gamma \mu(z-\zeta)=g \cdot \mu(z-\zeta) \cdot g^{-1}$ implies $\gamma\left(\hat{Z}_{\preceq \mu, E_{0}}\right)=\hat{Z}_{\preceq \mu, E_{0}}$. For the opposite inclusion $E_{\mu} \subset E_{\hat{Z}_{\preceq \mu}}$, note that every $\gamma \in \operatorname{Gal}\left(E_{0}\right.$ sep $\left./ \mathbb{F}_{q}((\zeta))\right)$ with $\gamma\left(\hat{Z}_{\preceq \mu}\right)=\hat{Z}_{\preceq \mu}$ satisfies $\hat{Z}_{\preceq \gamma \mu, E_{0}}=$ $\gamma\left(\hat{Z}_{\preceq \mu, E_{0}}\right)=\hat{Z}_{\preceq \mu, E_{0}}$. Because the Schubert varieties in $\operatorname{Gr}_{G, E_{0} \text { sep }}^{\mathbf{B}_{\mathrm{dR}}}$ are in bijection with the $G\left(E_{0}{ }^{\text {sep }}\right)$-conjugacy classes of cocharacters $\mathbb{G}_{m, E_{0}}$ sep $\rightarrow G_{E_{0}}$ sep, we conclude that $\gamma \mu$ is conjugate to $\mu$ and $\gamma$ lies in the group (2.6). Therefore, $E_{\mu} \subset E_{\hat{Z}_{\preceq \mu}}$, and our claim is proved.

We describe the generic fibre of $\hat{Z}$. Let $L$ be the completion of an algebraic closure of $E_{0}$. Choose a maximal torus $T$ of $G_{L}$ through which $\mu$ factors, a Borel subgroup $B \supset T$ with respect to which $\mu$ is dominant, and consider all dominant $\mu^{\prime} \in X_{*}(T)_{\text {dom }}$ with $\mu^{\prime} \preceq \mu$ in the Bruhat order. Then the $L$-valued points of $\hat{Z}^{\text {an }}$ lie in the union of the $G(L \llbracket z-\zeta \rrbracket)$-cosets

$$
\bigcup_{\mu^{\prime} \preceq \mu} G(L \llbracket z-\zeta \rrbracket) \cdot \mu^{\prime}(z-\zeta) \cdot G(L \llbracket z-\zeta \rrbracket) / G(L \llbracket z-\zeta \rrbracket) .
$$

Compare also Remark 2.11(b).

The special fibre of $\hat{Z}_{\preceq \mu}$ is discussed in [84, p. 3739 ff.] as a certain union of Schubert varieties.

Finally, because $\hat{Z}_{\preceq \mu}$ is defined as the closure of $\hat{Z}_{\preceq \mu, E_{0}}$, the bound $\left(\hat{Z}_{\preceq \mu}\right)^{-1}$ from Lemma 2.12 equals $\hat{Z}_{\preceq(-\mu)}$ where the cocharacter $-\mu: \mathbb{G}_{m, E_{0}} \rightarrow G_{E_{0}}$ is obtained from $\mu$ by precomposing with the inversion on $\mathbb{G}_{m, E_{0}}$.

Example 2.8. We explain the relation of boundedness in Definition 2.2 to the definition from [56]. Consider a split reductive group $G_{0}$ over $\mathbb{F}_{q}$, and set $G:=G_{0} \times_{\mathbb{F}_{q}} \operatorname{Spec} \mathbb{F}_{q} \llbracket z \rrbracket$. Let $T \subset G_{0}$ be a maximal split torus over $\mathbb{F}_{q}$. Let $B$ be a Borel subgroup containing $T$ and $\bar{B}$ its opposite Borel. Let $\mu \in X_{*}(T)_{\text {dom }}$ be a coweight that is dominant with respect to $B$. In [56, Definition 3.5], we define 'boundedness by $(\mu, z-\zeta)$ ' as follows. We consider a finite generating system $\Lambda$ of the monoid of dominant weights $X^{*}(T)_{\text {dom }}$, and for all $\lambda \in \Lambda$ the Weyl module $V_{\lambda}:=\left(\operatorname{Ind} \frac{G_{0}}{B}(-\lambda)_{\text {dom }}\right)^{\vee}$. Here $(-\lambda)_{\text {dom }}$ is the dominant representative in the Weyl group orbit of $-\lambda$. Let $\mathcal{G}$ and $\mathcal{G}^{\prime}$ be $L^{+} G$-torsors over a scheme 
$S \in \mathcal{N} i l p_{\mathbb{F}_{q} \llbracket \zeta \rrbracket}$ and let $\delta: L \mathcal{G} \stackrel{\sim}{\longrightarrow} L \mathcal{G}^{\prime}$ be an isomorphism of the associated $L G$-torsors. For the representation $\rho_{\lambda}: G \rightarrow \operatorname{GL}\left(V_{\lambda}\right)$ in $\operatorname{Rep}_{\mathbb{F}_{q} \llbracket z \rrbracket} G$, we consider the sheaves of $\mathcal{O}_{S} \llbracket z \rrbracket$ modules $\rho_{\lambda *} \mathcal{G}$ and $\rho_{\lambda *} \mathcal{G}^{\prime}$ associated with the $L^{+} G$-torsors $\mathcal{G}$ and $\mathcal{G}^{\prime}$ over $S$. The isomorphism $\delta$ induces an isomorphism $\rho_{\lambda *} \delta: \rho_{\lambda *} \mathcal{G} \otimes_{\mathcal{O}_{S} \llbracket z \rrbracket} \mathcal{O}_{S}((z)) \stackrel{\sim}{\longrightarrow} \rho_{\lambda *} \mathcal{G}^{\prime} \otimes_{\mathcal{O}_{S} \llbracket z \rrbracket} \mathcal{O}_{S}((z))$. After choosing trivialisations of $\mathcal{G}$ and $\mathcal{G}^{\prime}$ over an étale covering $S^{\prime} \rightarrow S$, the isomorphism $\delta$ is given by multiplication with an element $\delta_{S^{\prime}} \in L G\left(S^{\prime}\right)$. The latter corresponds to a morphism $S^{\prime} \rightarrow L G$. Let $L G_{\mu \#} \subset L G$ and $\mathcal{F}_{\mu \#} \subset \widehat{\mathcal{F} \ell_{G}}$ be the connected components corresponding to the image $\mu^{\#} \in \pi_{1}(G)_{I}=\pi_{1}(G)=\pi_{1}\left(G_{0}\right)=\pi_{0}\left(\widehat{\mathcal{F}} \ell_{G}\right)=\pi_{0}(L G)$ of $\mu$. According to [56, Definition 3.5], ' $\delta$ is bounded by $(\mu, z-\zeta)$ ' if

- the morphism $S^{\prime} \rightarrow L G$ factors through $L G_{\mu \#}$ and

- $\rho_{\lambda *} \delta\left(\rho_{\lambda *} \mathcal{G}\right) \subset(z-\zeta)^{-\left\langle(-\lambda)_{\mathrm{dom}}, \mu\right\rangle} \cdot \rho_{\lambda *} \mathcal{G}^{\prime}$ for all $\lambda \in \Lambda$.

In terms of Definition 2.2, this can be described as follows. Consider the ind-scheme

$$
Y_{\lambda}:=L \mathrm{GL}\left(V_{\lambda}\right) \widehat{\times}_{\mathbb{F}_{q}} \operatorname{Spf} \mathbb{F}_{q} \llbracket \zeta \rrbracket=\lim _{\longrightarrow} Y_{\lambda, m} \quad \text { for } \quad Y_{\lambda, m}:=L \mathrm{GL}\left(V_{\lambda}\right) \times_{\mathbb{F}_{q}} \operatorname{Spec} \mathbb{F}_{q} \llbracket \zeta \rrbracket /\left(\zeta^{m}\right)
$$

and let $M_{\lambda} \in L \mathrm{GL}\left(V_{\lambda}\right)\left(Y_{\lambda}\right)$ be the universal element over $Y_{\lambda}$. Because $\mathcal{O}_{Y_{\lambda, m}} \llbracket z \rrbracket\left[z^{-1}\right]=$ $\mathcal{O}_{Y_{\lambda, m}} \llbracket z-\zeta \rrbracket\left[\frac{1}{z-\zeta}\right]$, we can write $M_{\lambda}=\left(M_{\lambda, m}\right)_{m}$ with

$$
M_{\lambda, m} \in L \mathrm{GL}\left(V_{\lambda}\right)\left(Y_{\lambda, m}\right)=\operatorname{GL}\left(V_{\lambda}\right)\left(\mathcal{O}_{Y_{\lambda, m}} \llbracket z \rrbracket\left[z^{-1}\right]\right)=\operatorname{GL}\left(V_{\lambda}\right)\left(\mathcal{O}_{Y_{\lambda, m}} \llbracket z-\zeta \rrbracket\left[\frac{1}{z-\zeta}\right]\right) .
$$

Let $\bar{Y}_{\lambda} \subset Y_{\lambda}$ be the closed ind-subscheme where the matrix $(z-\zeta)^{\left\langle(-\lambda)_{\text {dom }}, \mu\right\rangle} M_{\lambda}$ has entries in $\mathcal{O}_{Y_{\lambda}} \llbracket z-\zeta \rrbracket=\mathcal{O}_{Y_{\lambda, m}} \llbracket z \rrbracket$ for all $m$, and let $\bar{Y}_{\lambda}^{\prime} \subset Y_{\lambda}$ be the closed ind-subscheme where the matrix $(z-\zeta)^{\left\langle(-\lambda)_{\text {dom }}, \mu\right\rangle} M_{\lambda}^{-1}$ has entries in $\mathcal{O}_{Y_{\lambda, m}} \llbracket z-\zeta \rrbracket=\mathcal{O}_{Y_{\lambda, m}} \llbracket z \rrbracket$ for all $m$. Set

$$
\begin{aligned}
\hat{Z}_{\preceq \mu, \lambda}^{\mathrm{Weyl}}: & =\bar{Y}_{\lambda} /\left(L^{+} \operatorname{GL}\left(V_{\lambda}\right) \widehat{\times}_{\mathbb{F}_{q}} \operatorname{Spf} \mathbb{F}_{q} \llbracket \zeta \rrbracket\right) \subset \widehat{\mathcal{F} \ell_{\mathrm{GL}}\left(V_{\lambda}\right)} \quad \text { and } \\
\hat{Z}_{\preceq \mu, \lambda}^{\mathrm{Weyl},-1}: & =\bar{Y}_{\lambda}^{\prime} /\left(L^{+} \operatorname{GL}\left(V_{\lambda}\right) \widehat{\times}_{\mathbb{F}_{q}} \operatorname{Spf} \mathbb{F}_{q} \llbracket \zeta \rrbracket\right) \subset \widehat{\mathcal{F} \ell_{\mathrm{GL}}\left(V_{\lambda}\right) .}
\end{aligned}
$$

Write $\Lambda=\left\{\lambda_{1}, \ldots, \lambda_{m}\right\}$ and for each $\lambda_{i}$ consider the morphism $\rho_{\lambda_{i} *}: \widehat{\mathcal{F}}_{G} \rightarrow \widehat{\mathcal{F}}_{\mathrm{GL}\left(V_{\lambda_{i}}\right)}$ induced from $\rho_{\lambda_{i}}$. Let $\hat{Z}_{G_{0}, \preceq \mu}^{\mathrm{Weyl}} \subset \mathcal{F}_{\mu^{\#}}$ be the base change of the closed ind-subscheme

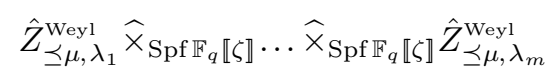

under the morphism $\prod_{i} \rho_{\lambda_{i} *}: \mathcal{F}_{\mu^{\#}} \rightarrow \widehat{\mathcal{F}}_{\mathrm{GL}\left(V_{\lambda_{1}}\right)} \widehat{\times}_{\mathbb{F}_{q} \llbracket \zeta \rrbracket} \ldots \widehat{\times}_{\mathbb{F}_{q} \llbracket \zeta \rrbracket} \widehat{\mathcal{F}}_{\mathrm{GL}\left(V_{\lambda_{m}}\right)}$. Likewise, let $\hat{Z}_{G_{0}, \preceq \mu}^{\text {Weyl,-1}} \subset \mathcal{F}_{-\mu^{\#}}$ be the base change of the closed ind-subscheme

$$
\hat{Z}_{\preceq \mu, \lambda_{1}}^{\mathrm{Weyl},-1} \widehat{\times}_{\operatorname{Spf} \mathbb{F}_{q} \llbracket \zeta \rrbracket} \cdots \widehat{\times}_{\operatorname{Spf} \mathbb{F}_{q} \llbracket \zeta \rrbracket} \hat{Z}_{\preceq \mu, \lambda_{m}}^{\text {Weyl, }-1}
$$

under the morphism $\prod_{i} \rho_{\lambda_{i} *}: \mathcal{F}_{-\mu^{\#}} \rightarrow \widehat{\mathcal{F}}_{\mathrm{GL}\left(V_{\lambda_{1}}\right)} \widehat{\times}_{\mathbb{F}_{q} \llbracket \zeta \rrbracket} \ldots \widehat{\times}_{\mathbb{F}_{q} \llbracket \zeta \rrbracket} \widehat{\mathcal{F}}_{\mathrm{GL}\left(V_{\lambda_{m}}\right)}$. The indsubscheme $\hat{Z}_{G_{0}, \preceq \mu}^{\mathrm{Weyl},-1} \subset \mathcal{F}_{-\mu^{\#}}$ was denoted $\widehat{\mathrm{Gr}}^{\preceq(\mu, z-\zeta)}$ in [56, Definition 5.5]. We will show that both $\hat{Z}_{G_{0}, \preceq \mu}^{\mathrm{Weyl}}$ and $\hat{Z}_{G_{0}, \preceq \mu}^{\mathrm{Weyl},-1}$ define bounds in the sense of Definition 2.2(b) 
with reflex ring $\mathbb{F}_{q} \llbracket \zeta \rrbracket$ and are representatives defined over the reflex ring. In terms of Lemma 2.12 , they satisfy $\hat{Z}_{G_{0}, \preceq \mu}^{\text {Weyl, } \_}=\left(\hat{Z}_{G_{0}, \preceq \mu}^{\text {Weyl }}\right)^{-1}$ and $\hat{Z}_{G_{0}, \preceq \mu}^{\text {Weyl }}=\left(\hat{Z}_{G_{0}, \preceq \mu}^{\text {Weyl,-1}}\right)^{-1}$. Conditions (b)(i), respectively (b)(v), are satisfied because all of the $\hat{Z}_{\preceq \mu, \lambda}^{\mathrm{Weyl}}$ and $\hat{Z}_{\preceq \mu, \lambda}^{\mathrm{Weyl},-1}$ are invariant by multiplication on the left with $L^{+} G$, respectively with $G(\bullet \llbracket z-\zeta \rrbracket)$. Conditions (b) (ii) and (b) (iii) for $\hat{Z}_{G_{0}, \preceq \mu}^{\mathrm{Weyl},-1}$ follow from [56, Proposition 5.5] and for $\hat{Z}_{G_{0}, \preceq \mu}^{\text {Weyl }}$ from Lemma 2.12. This proposition also says that the underlying reduced subscheme of $\hat{Z}_{G_{0}, \preceq \mu}^{\text {Weyl, } \_}$ is the closed Schubert variety associated with $(-\mu)_{\text {dom }}$ in $\mathcal{F} \ell_{G}$ and the underlying reduced subscheme of $\hat{Z}_{G_{0}, \preceq \mu}^{\text {Weyl }}$ is the closed Schubert variety associated with $\mu$. If $V:=\bigoplus_{\lambda \in \Lambda} V_{\lambda}$ and $\rho$ is the representation of $G_{0}$ on $V \oplus(\operatorname{det} V)^{\vee}$ which is faithful by [56, Proposition 3.14] and factors through $\mathrm{SL}_{1+\operatorname{dim} V}$, then $\hat{Z}_{G_{0}, \preceq \mu}^{\mathrm{Weyl}}$ is contained in $\widehat{\mathcal{F l}}_{\mathrm{SL}_{1+\operatorname{dim} V}^{(n)}}$ for $n=\max \left\{\left\langle(-\lambda)_{\text {dom }}, \mu\right\rangle: \lambda \in \Lambda\right\}$; that is, Condition (b)(iv) holds for $\hat{Z}_{G_{0}, \preceq \mu}^{\text {Weyl }}$. Then it also holds for $\hat{Z}_{G_{0}, \preceq \mu}^{\text {Weyl, } \_-1}$ by Lemma 2.12 .

Now $\delta$ 'is bounded by $(\mu, z-\zeta)$ ' in the sense of [56, Definition 3.5] if and only if the morphism $S^{\prime} \rightarrow L G \widehat{\times}_{\mathbb{F}_{q}} \operatorname{Spf} \mathbb{F}_{q} \llbracket \zeta \rrbracket \rightarrow \widehat{\mathcal{F}}_{G}$ given by $\delta_{S^{\prime}} \in L G\left(S^{\prime}\right)$ factors through $\hat{Z}_{G_{0}, \preceq \mu}^{\text {Weyl }}$; that is, if and only if $\delta$ is bounded by $\hat{Z}_{G_{0}, \preceq \mu}^{\mathrm{Weyl}}$ in the sense of Definition 2.2(d). This is the case if and only if the morphism $S^{\prime} \rightarrow L G \widehat{\times}_{\mathbb{F}_{q}} \operatorname{Spf} \mathbb{F}_{q} \llbracket \zeta \rrbracket \rightarrow \widehat{\mathcal{F}} \ell_{G}$ given by $\delta_{S^{\prime}}^{-1} \in L G\left(S^{\prime}\right)$ factors through $\hat{Z}_{G_{0}, \preceq \mu}^{\text {Weyl,-1}}$; that is, if and only if $\delta^{-1}$ is bounded by $\hat{Z}_{G_{0}, \preceq \mu}^{\text {Weyl,-1}}$. In particular, a local $G$-shtuka is bounded by $\hat{Z}_{G_{0}, \preceq \mu}^{\mathrm{Weyl}}$ in the sense of Definition $2.2(\mathrm{~d})$ if and only if it is 'bounded by $(\mu, z-\zeta)$ '.

For the constant split group $G=G_{0} \times_{\mathbb{F}_{q}} \operatorname{Spec} \mathbb{F}_{q} \llbracket z \rrbracket$, the double cosets occurring in the description of the sets of closed points of the generic and of the special fibre of bounds are both parametrised by the set $X_{*}(T)_{\text {dom }}$, and for our bound $\hat{Z}_{G_{0}, \preceq \mu}^{\text {Weyl }}$, both the generic fibre and the special fibre correspond to the union of all double cosets for $\mu^{\prime} \preceq \mu$. That is, the reduced generic, respectively the reduced special fibre of $\hat{Z}_{G_{0}, \preceq \mu}^{\mathrm{Weyl}}$, equals the closed Schubert variety associated with $\mu$ in $\operatorname{Gr}_{G}^{\mathbf{B}_{\mathrm{dR}}}$, respectively in $\mathcal{F} \ell_{G}$. So the underlying reduced structure of $\hat{Z}_{G_{0}, \preceq \mu}^{\text {Weyl }}$ coincides with the bound $\hat{Z}_{\preceq \mu}$ defined in Example 2.7, and in terms of Remark 2.11 condition (a) and $N_{0}=N_{\text {an }}$ from (b) are satisfied. The nilpotent structure as discussed in Remark 2.11(c) is in general not so clear in this case. For example, if $G_{0}=\mathrm{GL}_{r}$ and $\mu=2 n \rho^{\vee}$ where $2 \rho^{\vee}=(r-1, \ldots, 1-r)$ is the sum of the positive coroots

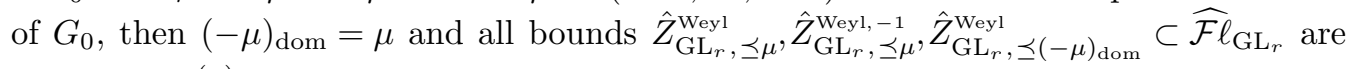
equal to $\widehat{\mathcal{F}}_{\mathrm{SL}_{r}}^{(n)} \subset \widehat{\mathcal{F} \ell_{\mathrm{SL}_{r}}} \subset \widehat{\mathcal{F} \ell_{\mathrm{GL}_{r}}}$ by [56, Lemma 4.3]. Note, however, that in general $\left(\hat{Z}_{G_{0}, \preceq \mu}^{\text {Weyl }}\right)^{-1}=\hat{Z}_{G_{0}, \preceq \mu}^{\text {Weyl,-1}}$ coincides with $\hat{Z}_{G_{0}, \preceq(-\mu)_{\text {dom }}}^{\text {Weyl }}$ only on the underlying reduced structure but not in the nilpotent structure; see Example 2.13.

Example 2.9. We give an example of an ind-scheme $\hat{Z}$ satisfying all properties of a bound in Definition 2.2 except for $(\mathrm{b})(\mathrm{v})$ to show that this condition is not implied by (b)(i). Let $\hat{Z} \subset \widehat{\mathcal{F}}_{\mathrm{SL}_{2}}^{(1)}$ be the ind-closure of $\hat{Y} \subset \widehat{\mathcal{F}}_{\mathrm{SL}_{2}}^{(1)}$ given by

$$
\begin{array}{r}
\hat{Y}(B):=L^{+} \mathrm{SL}_{2}(B) \cdot\left\{\left(\begin{array}{ll}
a & b \\
c & d
\end{array}\right) \in L \mathrm{SL}_{2}(B): a, b, d \in B \llbracket z \rrbracket, c \in \frac{\zeta}{z-\zeta} B \llbracket z \rrbracket\right\} \\
\cdot L^{+} \mathrm{SL}_{2}(B) / L^{+} \mathrm{SL}_{2}(B)
\end{array}
$$


for any $\mathbb{F}_{q} \llbracket \zeta \rrbracket$-algebra $B$. This satisfies all conditions in the definition of bounds except for possibly (b)(v). Notice further that the special fibre of $\hat{Z}$ consists just of the one point $L^{+} \mathrm{SL}_{2} / L^{+} \mathrm{SL}_{2}$.

We have that $x=\left(\begin{array}{cc}1 & 0 \\ \frac{\zeta}{z-\zeta} & 1\end{array}\right)$ and $y=\left(\begin{array}{cc}z-\zeta & 0 \\ 0 & \frac{1}{z-\zeta}\end{array}\right)$ are elements of $L \mathrm{SL}_{2}(B)$ for all $B$ such that $\zeta$ is nilpotent in $B$. Therefore, $x \in \hat{Z}\left(\operatorname{Spf} \mathbb{F}_{q} \llbracket \zeta \rrbracket\right)$ as one can see by reducing modulo $\left(\zeta^{i}\right)$ for all $i$. However, already considering the reduction modulo $\zeta$ shows that $y$ is not an element of $\hat{Z}\left(\mathbb{F}_{q} \llbracket \zeta \rrbracket\right)$. On the other hand,

$$
y=\left(\begin{array}{cc}
1 & -\zeta^{-1}(z-\zeta) \\
0 & 1
\end{array}\right) \cdot x \cdot\left(\begin{array}{cc}
z-\zeta & \zeta^{-1} \\
-\zeta & 0
\end{array}\right) \in \mathrm{SL}_{2}\left(\mathbb{F}_{q}((\zeta)) \llbracket z-\zeta \rrbracket\right) \cdot x \cdot \mathrm{SL}_{2}\left(\mathbb{F}_{q}((\zeta)) \llbracket z-\zeta \rrbracket\right) .
$$

This shows that $\hat{Z}^{\text {an }}$ is not invariant under multiplication with $\mathrm{SL}_{2}(\bullet \llbracket z-\zeta \rrbracket)$ on the left.

We need condition (b)(v) of Definition 2.2 mainly in form of the following lemma.

Lemma 2.10. Let $\hat{Z}$ be a bound. Then $\hat{Z}^{a n} \otimes_{E_{\hat{Z}}} \breve{E}_{\hat{Z}}$ is invariant under left and right multiplication with $L G(\mathbb{F})$.

Here, as always, we use the morphism $\mathbb{F}((z)) \rightarrow \mathcal{O}_{X} \llbracket z-\zeta \rrbracket, z \mapsto z=\zeta+(z-\zeta)$ for an

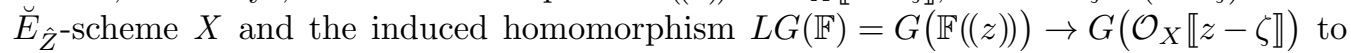
define the actions.

Proof. This follows directly from the right invariance of $\hat{Z}^{\text {an }}$ as a subscheme of the affine Grassmannian and the left invariance imposed in condition (b)(v) of Definition 2.2.

Remark 2.11. We discuss some possible additional assumptions on bounds.

(a) The set $\pi_{0}\left(\widehat{\mathcal{F}}_{G, R}\right)$ coincides with the set of $\Gamma$-orbits in $\pi_{1}(G)_{I}$ by [76, Lemma 2.2.6]. Every bound is a disjoint union of its intersections with the various connected components of $\widehat{\mathcal{F}}_{G, R}$, and thus we also obtain similar decompositions of base schemes for bounded local $G$-shtukas and later of the corresponding moduli spaces. If one wants to consider only one of these disjoint parts at a time, one has to assume that there is a $\Gamma$-orbit of elements $\xi \in \pi_{1}(G)_{I}$ such that the $\hat{Z}_{R}$ are contained in the corresponding connected component of $\widehat{\mathcal{F}}_{G, R}$.

Also compare the nonemptiness condition of Theorem 4.20, which is in terms of $\pi_{1}(G)_{\Gamma}$ instead of $\pi_{1}(G)_{I} / \Gamma$. The natural projection map $\pi_{1}(G)_{I} / \Gamma \rightarrow \pi_{1}(G)_{\Gamma}$ is surjective but in general not injective. Thus, there may be several connected components of a given bound that lead to nonempty parts of the period domain.

(b) If one wants to compare properties of the generic and the special fibre of a moduli space of local $G$-shtukas bounded by $\hat{Z}^{-1}$ (as defined in Section 3), it might be useful to consider bounds $\hat{Z}$ satisfying certain flatness or extension properties.

Assumptions (b)(i) and (b)(v) of Definition 2.2 imply that the closed points of the special fibre $Z$ from Remark 2.3(b), respectively of the analytic space $\hat{Z}^{\text {an }}$ of $\hat{Z}$ from Proposition 2.6(d), consist of the points of a finite set $N_{0}$ of $L^{+} G$-cosets, respectively a finite set $N_{\text {an }}$ of $G(\bullet \llbracket z-\zeta \rrbracket)$-cosets. The former cosets are parametrised by some quotient $\bar{W}$ of the extended affine Weyl group $\widetilde{W}$ of $G$ with respect to a chosen maximal torus $T$ of $G_{\mathbb{F}_{q}((z))}$. Let $L_{0} \supset \mathbb{F}_{q}((\zeta))$ be a finite separable field 
extension over which $T_{L_{0}}:=T \times_{\mathbb{F}_{q}((z)), z \mapsto \zeta} \operatorname{Spec} L_{0}$ (and therefore also $G_{L_{0}}$ ) splits. Because $G\left(L_{0} \llbracket z-\zeta \rrbracket\right) \subset G\left(L_{0}((z-\zeta))\right)$ is a hyperspecial maximal bounded open subgroup, every $G\left(L_{0} \llbracket z-\zeta \rrbracket\right)$-coset is of the form $G\left(L_{0} \llbracket z-\zeta \rrbracket\right) \mu(z-\zeta) G\left(L_{0} \llbracket z-\zeta \rrbracket\right) /$ $G\left(L_{0} \llbracket z-\zeta \rrbracket\right)$ for a uniquely determined determined $\mu \in X_{*}\left(T_{L_{0}}\right)_{\mathrm{dom}}=X_{*}(T)_{\mathrm{dom}}$ where dominance is with respect to some chosen Borel subgroup. It would thus be interesting to see whether such flatness conditions can be formulated in terms of the associated sets $N_{0} \subset \bar{W}$ and $N_{\text {an }} \subset X_{*}(T)_{\text {dom. }}$. However, for the present article we do not need any such condition.

(c) Given the discussion above, one might ask whether bounds should just be defined by the corresponding sets of double cosets of their geometric points. Even under assumptions as discussed above, our definition gives some more freedom in the sense that the nilpotent structure of the bound may still vary.

For the sake of completeness, we want to add the following lemma that was used in Remark 2.3(a).

Lemma 2.12. Let $\hat{Z}=\left[\left(R, \hat{Z}_{R}\right)\right]$ be a bound in the sense of [4, Definition 4.8]; that is, satisfying conditions (b)(i) and (b)(ii) from Definition 2.2. Consider the subsheaves $\hat{Z}_{R}^{-1} \subset \widehat{\mathcal{F}}_{G, R}$ defined on schemes $S$ in $\mathcal{N}$ ilp $p_{R}$ as the subset $\hat{Z}_{R}^{-1}(S)$ of $\widehat{\mathcal{F}}_{G, R}(S)$ given by

$\left\{x \in \widehat{\mathcal{F}}_{G, R}(S):\right.$ there is an étale covering $f: S^{\prime} \rightarrow S$ and an element $g \in L G\left(S^{\prime}\right)$ such that $f^{*} x=g \cdot L^{+} G\left(S^{\prime}\right)$ in $\widehat{\mathcal{F}}_{G, R}\left(S^{\prime}\right)$ and $\left.g^{-1} \cdot L^{+} G\left(S^{\prime}\right) \in \hat{Z}_{R}\left(S^{\prime}\right)\right\}$.

Then $\hat{Z}^{-1}=\left[\left(R, \hat{Z}_{R}^{-1}\right)\right]$ is a bound in the sense of [4, Definition 4.8] with the same reflex ring $R_{\hat{Z}}$ as $\hat{Z}$. If, in addition, $\hat{Z}$ satisfies condition (b)(iii) and (b)(iv) from Definition 2.2, then the same is true for $\hat{Z}^{-1}$. Moreover, for two $L^{+} G$-torsors $\mathcal{G}$ and $\mathcal{G}^{\prime}$ over a scheme $S \in \mathcal{N}$ ilp $_{R_{\hat{Z}}}$, an isomorphism $\delta: L \mathcal{G} \stackrel{\sim}{\longrightarrow} \mathcal{G}^{\prime}$ between the associated $L G$-torsors is bounded by $\hat{Z}$ if and only if $\delta^{-1}$ is bounded by $\hat{Z}^{-1}$.

We do not know whether the same is true for condition (b)(v) from Definition 2.2 in general. It is true, however, for the bounds in Example 2.7.

Proof of Lemma 2.12. The subset is well defined, because $\hat{Z}_{R}$ is by Definition 2.2(b)(i) invariant under multiplication with $L^{+} G$ on the left. We fix a faithful representation $\rho: G \hookrightarrow \mathrm{SL}_{r}$ and consider the induced morphism $\rho_{*}: \mathcal{F} \ell_{G} \hookrightarrow \mathcal{F} \ell_{\mathrm{SL}_{r}}$. The ind-scheme structure on $\mathcal{F} \ell_{G, R}$ is given as the inductive limit of the schemes

$$
X_{n}:=X_{n, n} \quad \text { with } \quad X_{n, m}:=\widehat{\mathcal{F}}_{G} \widehat{\times}_{\widehat{\mathcal{F}}_{\mathrm{SL}}} \widehat{\mathcal{F}}_{\mathrm{SL}_{r}}^{(n)} \widehat{\times}_{\mathbb{F}_{q} \llbracket \zeta} \operatorname{Spec} R /\left(\zeta^{m}\right),
$$

where $\widehat{\mathcal{F}}_{\mathrm{SL}_{r}}^{(n)}$ was defined in (2.1). We must show that $\hat{Z}_{R}^{-1} \times_{\widehat{\mathcal{F}}_{G, R}} X_{n}$ is representable by a closed subscheme of $X_{n}$. Let $Y_{n}:=X_{n} \widehat{\times}_{\widehat{\mathcal{F}}_{G, R}}\left(L G \widehat{\times}_{\mathbb{F}_{q}} \operatorname{Spec} R /\left(\zeta^{n}\right)\right)$. Because 
$X_{n} \widehat{\times}_{\widehat{\mathcal{F}}_{G, R}} \hat{Z}_{R} \subset X_{n}$ is a closed subscheme, the base change $Z_{n}:=Y_{n} \widehat{\times}_{X_{n}} X_{n} \widehat{\times}_{\widehat{\mathcal{F}}_{G, R}} \hat{Z}_{R}$ is a closed subscheme of $Y_{n}$, on which $L^{+} G_{R /\left(\zeta^{n}\right)}:=L^{+} G \widehat{\times}_{\mathbb{F}_{q}} \operatorname{Spec} R /\left(\zeta^{n}\right)$ acts by multiplication on the left. By Cramer's rule (e.g., [22, III.8.6, Formulas (21) and (22)]), the inversion $g \mapsto g^{-1}$ on $L G$ induces isomorphisms of $X_{n}$ with the quotient sheaf $L^{+} G_{R /\left(\zeta^{n}\right)} \backslash Y_{n}$ and of $\hat{Z}_{R}^{-1} \times \widehat{\mathcal{F}}_{G, R} X_{n}$ with $L^{+} G_{R /\left(\zeta^{n}\right)} \backslash Z_{n}$. Therefore, it suffices to show that the morphism $L^{+} G_{R /\left(\zeta^{n}\right)} \backslash Z_{n} \rightarrow L^{+} G_{R /\left(\zeta^{n}\right)} \backslash Y_{n}$ of sheaves is representable by a closed immersion. The latter follows by $f p q c$ descent $[20, \S 6.1$, Theorem 6$]$ and $\left[44, \mathrm{IV}_{2}\right.$, Proposition 2.7.1] from the fact that the diagram

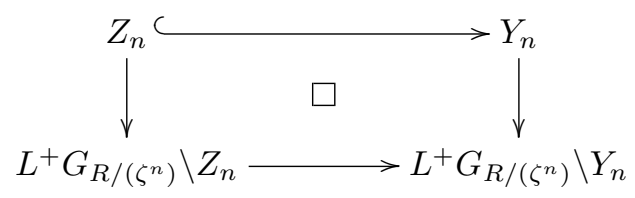

is Cartesian and the right vertical arrow is an affine faithfully flat morphism of schemes. This proves that $\hat{Z}_{R}^{-1} \subset \widehat{\mathcal{F}} \ell_{G, R}$ is representable by a closed ind-subscheme. By construction it satisfies the invariance under left multiplication by $L^{+} G$ from Definition 2.2(b)(i).

To show that $\hat{Z}_{R}^{-1}$ satisfies Definition 2.2(b)(ii), let $S^{\prime}$ be an étale covering of the special fibre $Z_{R}$ of $\hat{Z}_{R}$, such that the closed immersion $Z_{R} \hookrightarrow \widehat{\mathcal{F}}_{G, R} \widehat{\times}_{R} \operatorname{Spec} \kappa_{R}$ lifts to a morphism $S^{\prime} \rightarrow L G \widehat{\times}_{\mathbb{F}_{q}}$ Spec $\kappa_{R}$, which we view as an element $g^{-1} \in L G\left(S^{\prime}\right)$. Because $Z_{R}$ is quasi-compact, we may choose $S^{\prime}$ to be quasi-compact. Then the element $g \cdot L^{+} G\left(S^{\prime}\right)$ in $\widehat{\mathcal{F}}_{G, R}\left(S^{\prime}\right)$ corresponds to a morphism $S^{\prime} \rightarrow \widehat{\mathcal{F}}_{G, R} \widehat{\times}_{R} \operatorname{Spec} \kappa_{R}$ that factors through $Z_{R}^{-1}$. The morphism $L^{+} G \widehat{\times}_{\mathbb{F}_{q}} S^{\prime} \rightarrow Z_{R}^{-1},(h, g) \mapsto h g \cdot L^{+} G$ is a surjective morphism of ind-schemes. Therefore, $Z_{R}^{-1}$ is a quasi-compact scheme.

If $\hat{Z}$ satisfies conditions (b)(iii) and (b)(iv) from Definition 2.2 for some $\rho: G \hookrightarrow \mathrm{SL}_{r}$ and some $n$, then $\hat{Z}^{-1}$ also satisfies (b)(iv) for the same $\rho$ and $n$ by Cramer's rule (e.g., $\left[22\right.$, III.8.6, Formulas (21) and (22)]). Then $\hat{Z}_{R}^{-1}=\lim _{\longrightarrow} \hat{Z}_{R}^{-1} \times \widehat{\mathcal{F}}_{G, R} X_{n, m}$ for constant $n$ and variable $m$. In particular, $\hat{Z}_{R}^{-1}=\lim _{\longrightarrow} \hat{Z}_{R}^{-1} \times{ }_{R} \operatorname{Spec} R /\left(\zeta^{m}\right)$ is $\zeta$-adic; that is, satisfies condition (b)(iii)

The equality of reflex rings follows from the fact that $\gamma(\hat{Z})=\hat{Z}$ if and only if $\gamma\left(\hat{Z}^{-1}\right)=$ $\hat{Z}^{-1}$ for $\gamma \in \operatorname{Aut}_{\mathbb{F}_{q} \llbracket \zeta \rrbracket}\left(\mathbb{F}_{q}((\zeta))^{\text {alg }}\right)$. Finally, the statement about the boundedness of $\delta$ and $\delta^{-1}$ is clear from the definition of the $\hat{Z}_{R}^{-1}$.

Example 2.13. We revert to Example 2.8. If $G_{0}=\mathrm{GL}_{r}$ or $G_{0}=\mathrm{SL}_{r}$, then

$$
\hat{Z}_{G_{0}, \preceq \mu}^{\mathrm{Weyl}}=\left(\hat{Z}_{G_{0}, \preceq \mu}^{\mathrm{Weyl},-1}\right)^{-1} \stackrel{!}{=} \hat{Z}_{G_{0}, \preceq(-\mu)_{\mathrm{dom}}}^{\mathrm{Weyl},-1}
$$

by Cramer's rule (e.g., [22, III.8.6, Formulas (21) and (22)]). However, this is not true for general $G_{0}$. For example, let $G_{0}=\mathrm{PGL}_{2}$ and $\operatorname{char}\left(\mathbb{F}_{q}\right)=2$. We choose $\Lambda$ to consist of the only positive root $\alpha$. The corresponding Weyl module $V_{\alpha}$ is the dual of the adjoint 
representation. With respect to the decomposition $V_{\alpha}=\left(\operatorname{Lie} T \oplus \operatorname{Lie} U_{\alpha} \oplus \operatorname{Lie} U_{-\alpha}\right)^{\vee}$ it is given by

$$
\rho_{\alpha}: \mathrm{PGL}_{2} \rightarrow \mathrm{GL}\left(V_{\alpha}\right), \quad g=\left(\begin{array}{cc}
a & b \\
c & d
\end{array}\right) \mapsto\left(\begin{array}{ccc}
1 & \frac{a c}{\operatorname{det} g} & \frac{b d}{\operatorname{det} g} \\
0 & \frac{a^{2}}{\operatorname{det} g} & \frac{b^{2}}{\operatorname{det} g} \\
0 & \frac{c^{2}}{\operatorname{det} g} & \frac{d^{2}}{\operatorname{det} g}
\end{array}\right) .
$$

We let $\mu \in X_{*}(T)_{\text {dom }}$ be the dominant coweight with $\mu(a)=\left(\begin{array}{ll}a & 0 \\ 0 & 1\end{array}\right)$. Then $(-\mu)_{\mathrm{dom}}=\mu$ and

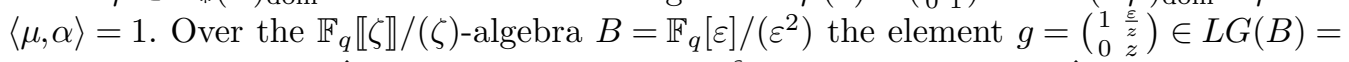
$\mathrm{PGL}_{2}(B((z)))$ lies in $\hat{Z}_{\mathrm{PGL}_{2}, \preceq \mu}^{\mathrm{Weyl},-1}$, but $g^{-1}=\left(\begin{array}{cc}z-\frac{\varepsilon}{z} \\ 0 & 1\end{array}\right)$ does not belong to $\hat{Z}_{\mathrm{PGL}_{2}, \preceq \mu}^{\mathrm{Weyl},-1}$ because

$$
\rho_{\alpha}(g)=\left(\begin{array}{ccc}
1 & 0 & \frac{\varepsilon}{z} \\
0 & \frac{1}{z} & 0 \\
0 & 0 & z
\end{array}\right) \quad \text { and } \quad \rho_{\alpha}\left(g^{-1}\right)=\left(\begin{array}{ccc}
1 & 0 & -\frac{\varepsilon}{z^{2}} \\
0 & z & 0 \\
0 & 0 & \frac{1}{z}
\end{array}\right)
$$

So $\hat{Z}_{\mathrm{PGL}_{2}, \preceq \mu}^{\mathrm{Weyl}} \neq \hat{Z}_{\mathrm{PGL}_{2}, \preceq(-\mu)_{\text {dom }}}^{\mathrm{Weyl},-1}$ in this case. Note that, nevertheless, the underlying topological spaces of these two bounds coincide by Remark 2.11(b). So the difference lies in the nilpotent structure.

\section{Rapoport-Zink spaces for bounded local $G$-shtukas}

To recall the definition of Rapoport-Zink spaces for local $G$-shtukas, let $\underline{\mathbb{G}}_{0}$ be a local $G$-shtuka over $\mathbb{F}$. Because $\mathbb{F}$ has no nontrivial étale coverings, we may fix a trivialisation $\underline{\mathbb{G}}_{0} \cong\left(\left(L^{+} G\right)_{\mathbb{F}}, b \sigma^{*}\right)$ where $b \in L G(\mathbb{F})$ represents the Frobenius morphism. In all that follows we may replace $\underline{\mathbb{G}}_{0}$ by a quasi-isogenous local $G$-shtuka $\underline{\mathbb{G}}_{0}^{\prime} \cong\left(\left(L^{+} G\right)_{\mathbb{F}}, b^{\prime} \sigma^{*}\right)$. In terms of the trivialisations, this means that there is an $h \in L G(\mathbb{F})$ with $b^{\prime}=h^{-1} b \sigma^{*}(h)$. In this case we say that $b$ and $b^{\prime}$ are $\sigma$-conjugate under $L G(\mathbb{F})$ or $L G(\mathbb{F})$ - $\sigma$-conjugate. We write $[b]$ for the $L G(\mathbb{F})-\sigma$-conjugacy class of $b$.

Definition 3.1. Let $\hat{Z}=\left[\left(R, \hat{Z}_{R}\right)\right]$ be a bound with reflex $\operatorname{ring} R_{\hat{Z}}=\kappa \llbracket \xi \rrbracket$, set $\breve{R}_{\hat{Z}}:=\mathbb{F} \llbracket \xi \rrbracket$ and consider the functor $\breve{\mathcal{M}}_{\underline{\mathbb{G}}_{0}}^{\hat{Z}^{-1}}:\left(\mathcal{N} i l p_{\breve{R}_{\hat{Z}}}\right)^{o} \longrightarrow$ Sets

$S \longmapsto\{$ Isomorphism classes of $(\underline{\mathcal{G}}, \bar{\delta})$ : where $\underline{\mathcal{G}}$ is a local $G$-shtuka over $S$ bounded by $\hat{Z}^{-1}$ and $\bar{\delta}: \underline{\mathcal{G}}_{\bar{S}} \rightarrow \underline{\mathbb{G}}_{0, \bar{S}}$ is a quasi-isogeny over $\left.\bar{S}\right\}$.

Here $\bar{S}:=\mathrm{V}_{S}(\zeta)$ is the zero locus of $\zeta$ in $S$.

The group QIsog ${ }_{\mathbb{F}}\left(\underline{\mathbb{G}}_{0}\right)$ of quasi-isogenies of $\underline{\mathbb{G}}_{0}$ acts on the functor $\breve{\mathcal{M}}_{\underline{\mathbb{G}}_{0}}^{\hat{Z}^{-1}}$ via $j:(\underline{\mathcal{G}}, \bar{\delta}) \mapsto$ $(\underline{\mathcal{G}}, j \circ \bar{\delta})$ for $j \in \mathrm{QIsog}_{\mathbb{F}}\left(\underline{\mathbb{G}}_{0}\right)$.

Remark 3.2. Because $\underline{\mathbb{G}}_{0} \cong\left(\left(L^{+} G\right)_{\mathbb{F}}, b \sigma^{*}\right)$, we can identify $\operatorname{QIsog}_{\mathbb{F}}\left(\underline{\mathbb{G}}_{0}\right) \cong J_{b}\left(\mathbb{F}_{q}((z))\right)$ where $J_{b}$ is the connected algebraic group over $\mathbb{F}_{q}((z))$ that is defined by its functor of 
points that assigns to an $\mathbb{F}_{q}((z))$-algebra $A$ the group

$$
J_{b}(A):=J_{b}^{G}(A):=\left\{g \in G\left(A \otimes_{\mathbb{F}_{q}((z))} \mathbb{F}((z))\right): g^{-1} b \sigma^{*}(g)=b\right\} ;
$$

see [4, Remark 4.16].

Remark 3.3. As in the arithmetic case (compare [80, 3.48]), we have a Weil descent datum $\alpha$ on the functor $\breve{\mathcal{M}}_{\mathbb{G}_{0}}^{\hat{Z}^{-1}}$. To define it, let $S \in \mathcal{N} i l p_{\breve{R}_{\hat{Z}}}$ and let $f: S \rightarrow \operatorname{Spf} \breve{R}_{\hat{Z}}$ and $\bar{f}: \bar{S} \rightarrow \operatorname{Spec} \breve{R}_{\hat{Z}} /(\zeta)$ be the structure morphisms. Let $\varphi$ be the Frobenius of $\breve{R}_{\hat{Z}}=\mathbb{F} \llbracket \xi \rrbracket$ over $R_{\hat{Z}}=\kappa \llbracket \xi \rrbracket$ and let $S_{\varphi}$ be the scheme $S$ together with the structure morphism $\varphi \circ f: S \rightarrow$ Spf $\breve{R}_{\hat{Z}}$. If \# $\#=q^{e}$, the inclusion $\mathbb{F} \hookrightarrow \breve{R}_{\hat{Z}}$ is equivariant for the action of $\varphi$ on $\breve{R}_{\hat{Z}}$ and the action of $\sigma^{e}$ on $\mathbb{F}$. To define $\alpha: \breve{\mathcal{M}}_{\mathbb{\mathbb { G }}_{0}}^{\hat{Z}^{-1}}(S) \stackrel{\sim}{\sim}\left(\varphi^{*} \breve{\mathcal{M}}_{\mathbb{\mathbb { G }}_{0}}^{\hat{Z}^{-1}}\right)(S):=\breve{\mathcal{M}}_{\mathbb{\mathbb { G }}_{0}}^{\hat{Z}^{-1}}\left(S_{\varphi}\right)$, let $(\underline{\mathcal{G}}, \bar{\delta})$ be a point in the first set. To make the definition more clear, we write $\underline{\mathbb{G}}_{0, \bar{S}}=\bar{f}^{*}\left(\underline{\mathbb{G}}_{0}\right)$. Then we set $\alpha(\underline{\mathcal{G}}, \bar{\delta})=\left(\underline{\mathcal{G}}, \bar{f}^{*}\left(\tau_{\mathbb{G}_{0}}^{e}\right)^{-1} \circ \bar{\delta}\right)$; that is, we replace the quasi-isogeny $\bar{\delta}$ by the composite $\underline{\mathcal{G}}_{\bar{S}} \rightarrow \bar{f}^{*}\left(\underline{\mathbb{G}}_{0}\right) \rightarrow \bar{f}^{*} \sigma^{e *}\left(\underline{\mathbb{G}}_{0}\right)=(\bar{\varphi} \bar{f})^{*}\left(\underline{\mathbb{G}}_{0}\right)$. Although this Weil descent datum is in general not effective, $\breve{\mathcal{M}}_{\mathbb{G}_{0}}^{\hat{Z}^{-1}}$ always descends to a finite unramified extension of $\breve{R}_{\hat{Z}}$; see Remark 3.6(b).

Remark 3.4. By its definition, $\breve{\mathcal{M}}_{\mathbb{G}_{0}}^{\hat{Z}^{-1}}$ only depends on the triple $(G,[b], \hat{Z})$, where $[b]$ is the $L G(\mathbb{F})-\sigma$-conjugacy class of $b$ corresponding to the isogeny class of $\underline{\mathbb{G}}_{0} \cong$ $\left(\left(L^{+} G\right)_{\mathbb{F}}, b \sigma^{*}\right)$. In the arithmetic analogue, the corresponding independence of the Rapoport-Zink spaces on additional choices made in their definition is far from obvious. It was conjectured in [79, Conjecture 4.16] and proved in [90, Corollary 23.4.3 and thereafter].

As in Remark 2.3(a) and (b), we let $\hat{Z}^{-1}$ be the bound from Lemma 2.12, and we let $Z^{-1}$ be the special fibre of $\hat{Z}^{-1}$ over $\kappa$. We define the associated affine Deligne-Lusztig variety as the reduced closed ind-subscheme $X_{Z^{-1}}(b) \subset \mathcal{F} \ell_{G}$ whose $K$-valued points (for any field extension $K$ of $\mathbb{F}$ ) are given by

$$
X_{Z^{-1}}(b)(K):=\left\{g \in \mathcal{F} \ell_{G}(K): g^{-1} b \sigma^{*}(g) \in Z^{-1}(K)\right\} .
$$

In [4, Theorem 4.18 and Corollary 4.26], the following theorem was proved.

Theorem 3.5. The functor $\breve{\mathcal{M}}_{\underline{\mathbb{G}}_{0}}^{\hat{Z}^{-1}}:\left(\mathcal{N} i l p_{\breve{R}_{\hat{Z}}}\right)^{o} \rightarrow$ Sets from Definition 3.1 is indrepresentable by a formal scheme over $\operatorname{Spf} \breve{R}_{\hat{Z}}$ which is locally formally of finite type and separated. It is an ind-closed ind-subscheme of $\mathcal{F} \ell_{G} \widehat{\times}_{\mathbb{F}_{q}}$ Spf $\breve{R}_{\hat{Z}}$. Its underlying reduced subscheme equals $X_{Z^{-1}}(b)$, which is a scheme locally of finite type and separated over $\mathbb{F}$, all of whose irreducible components are projective.

The formal scheme representing $\breve{\mathcal{M}}_{\mathbb{G}_{0}}^{\hat{Z}^{-1}}$ is called a Rapoport-Zink space for bounded local G-shtukas. Recall that a formal scheme over $\breve{R}_{\hat{Z}}$ in the sense of [44, $\left.\mathrm{I}_{\text {new }}, 10\right]$ is called locally formally of finite type if it is locally Noetherian and adic and its reduced subscheme is locally of finite type over $\mathbb{F}$.

Remark 3.6. (a) $L G(\mathbb{F})$ - $\sigma$-conjugacy classes in $L G(\mathbb{F})$ are in bijection to isogeny classes of local $G$-shtukas over $\mathbb{F}$. To classify them, Kottwitz associated with every element 
$b \in L G(\mathbb{F})$ a slope homomorphism $\nu_{b}: \mathbb{D}_{\mathbb{F}((z))} \rightarrow G_{\mathbb{F}((z))}$, called Newton point (or Newton polygon) of $b$; see $[70,4.2]$. Here $\mathbb{D}$ is the diagonalisable pro-algebraic group over $\mathbb{F}((z))$ with character group $\mathbb{Q}$. The slope homomorphism is characterised by assigning the slope filtration of

$$
\left(V \otimes_{\mathbb{F}_{q}((z))} \mathbb{F}((z)), \rho(b) \sigma^{*}\right)
$$

to any $(V, \rho)$ in $\operatorname{Rep}_{\mathbb{F}_{q}((z))} G$; see [70, Section 4]. Furthermore, he showed that $\sigma$-conjugating $b$ amounts to conjugating $\nu_{b}$ by the corresponding element. One can thus associate with the $L G(\mathbb{F})$ - $\sigma$-conjugacy class $[b]$ a well-defined $G(\mathbb{F}((z)))$-conjugacy class $\left\{\nu_{b}\right\}$ in $\operatorname{Hom}\left(\mathbb{D}_{\mathbb{F}((z))}, G_{\mathbb{F}((z)))}\right)$, which moreover is invariant under $\sigma$ by $[70,4.4]$.

The second important invariant of $[b]$ is defined as follows. Consider again the Kottwitz homomorphism $\kappa_{G}: L G(\mathbb{F}) \rightarrow \pi_{1}(G)_{I}$ as explained in [77, 2.a.2]. We compose $\kappa_{G}$ with the projection to $\pi_{1}(G)_{\Gamma}$. This then yields a well-defined map (again denoted $\kappa_{G}$ ) from the set of $\sigma$-conjugacy classes $B(G)$ to $\pi_{1}(G)_{\Gamma}$ (see [71]). Together, $\left\{\nu_{b}\right\}$ and $\kappa_{G}([b])$ determine $[b]$ uniquely. We denote by $[b]^{\#}$ the images of $\kappa_{G}(b)$ in $\pi_{1}(G)_{\Gamma}$ and in $\pi_{1}(G)_{\Gamma, \mathbb{Q}}:=\pi_{1}(G)_{\Gamma} \otimes_{\mathbb{Z}} \mathbb{Q}$. The latter equals the image of the conjugacy class $\left\{\nu_{b}\right\}$ in $\pi_{1}(G)_{\Gamma, \mathbb{Q}}$; see [78, Theorem $\left.1.15(\mathrm{iii})\right]$.

(b) Because $\mathbb{F}$ is algebraically closed and the generic fibre of $G$ is connected reductive, we may replace $b$ by $h^{-1} b \sigma^{*}(h)$ and assume that $b \in L G(\mathbb{F})$ satisfies a decency equation for a positive integer $s$; that is, $s \nu_{b}: \mathbb{D}_{\mathbb{F}((z))} \rightarrow G_{\mathbb{F}((z))}$ factors through $\mathbb{G}_{m}$, and

$$
(b \sigma)^{s}=s \nu_{b}(z) \sigma^{s} \quad \text { in } \quad L G(\mathbb{F}) \rtimes\langle\sigma\rangle ;
$$

see [70, Section 4]. Let $\mathbb{F}_{q^{s}} \subset \mathbb{F}$ be the finite field extension of $\mathbb{F}_{q}$ of degree $s$. Then $b \in L G\left(\mathbb{F}_{q^{s}}\right), \nu_{b}$ is defined over $\mathbb{F}_{q^{s}}((z))\left(\left[80\right.\right.$, Corollary 1.9]) and $J_{b} \times_{\mathbb{F}_{q}((z))} \mathbb{F}_{q^{s}}((z))$ is an inner form of the centraliser of the 1-parameter subgroup $s \nu_{b}$ of $G$, a Levi subgroup of $G_{\mathbb{F}_{q} s((z))}$; see [80, Corollary 1.14]. In particular, $J_{b}\left(\mathbb{F}_{q}((z))\right) \subset G\left(\mathbb{F}_{q^{s}}((z))\right)=L G\left(\mathbb{F}_{q^{s}}\right)$. Moreover, in this case $\breve{\mathcal{M}}_{\mathbb{G}_{0}}^{\hat{Z}^{-1}}$ descends by [4, Theorem 4.18] to a formal scheme locally formally of finite type over $\left(\mathbb{F}_{q^{s}} \cdot \kappa\right) \llbracket \xi \rrbracket$ where $\mathbb{F}_{q^{s}} \cdot \kappa$ is the compositum inside $\mathbb{F}$.

(c) If more generally we start with a local $G$-shtuka $\underline{\mathbb{G}}_{0}$ over any field $k$ in $\mathcal{N} i l p_{R_{\hat{z}}}$, we can define the Rapoport-Zink functor $\mathcal{M}_{\mathbb{G}_{0}}^{\hat{Z}^{-1}}$ as in Definition 3.1 on the category $\mathcal{N} i l p_{k \llbracket \xi \rrbracket}$. Then $\underline{\mathbb{G}}_{0} \otimes_{k} k^{\text {alg }}$ is trivial and decent and hence $\mathcal{M}_{\mathbb{\mathbb { G }}_{0}}^{\hat{Z}^{-1}} \otimes_{k} k^{\text {alg }}$ is ind-representable by a formal scheme locally formally of finite type over $k^{\text {alg }} \llbracket \xi \rrbracket$. By an unpublished result of Eike Lau on Galois descent of formal schemes locally formally of finite type, already $\mathcal{M}_{\mathbb{\mathbb { G }}_{0}}^{\hat{Z}^{-1}}$ is ind-representable by a formal scheme locally formally of finite type over $k \llbracket \xi \rrbracket$. However, we will not use this in the rest of this work.

Remark 3.7. The constructions described above are functorial with respect to the group scheme $G$. To explain this, let $\varepsilon: G \rightarrow G^{\prime}$ be a morphism of parahoric group schemes over $\mathbb{F}_{q} \llbracket z \rrbracket$. Important examples are closed immersions, epimorphisms or the change of the parahoric model, that is, morphisms that are generically isomorphisms. Then $\varepsilon$ induces a functor

$$
\varepsilon_{*}:\{\text { local } G \text {-shtukas }\} \longrightarrow\left\{\text { local } G^{\prime} \text {-shtukas }\right\}, \underline{\mathcal{G}} \longmapsto \varepsilon_{*} \underline{\mathcal{G}}:=\underline{\mathcal{G}} \stackrel{G}{\times} G^{\prime} .
$$


The morphism $\varepsilon$ also induces morphisms $\varepsilon: L^{+} G \rightarrow L^{+} G^{\prime}$ and $\varepsilon: L G \rightarrow L G^{\prime}$ and $\varepsilon: \mathcal{F} \ell_{G} \rightarrow \mathcal{F} \ell_{G^{\prime}}$. We say that $\varepsilon$ is compatible with two given bounds $\hat{Z}=\left[\left(R, \hat{Z}_{R}\right)\right]$ and $\hat{Z}^{\prime}=\left[\left(R, \hat{Z}_{R}^{\prime}\right)\right]$ with $\hat{Z}_{R} \subset \widehat{\mathcal{F}}_{G, R}$ and $\hat{Z}_{R}^{\prime} \subset \widehat{\mathcal{F}}_{G^{\prime}, R}$ if $\varepsilon\left(\hat{Z}_{R}\right) \subset \hat{Z}_{R}^{\prime}$ for all suitable $R$. If $\varepsilon$ is compatible with $\hat{Z}$ and $\hat{Z}^{\prime}$ and $\underline{\mathcal{G}}$ is bounded by $\hat{Z}^{-1}$, then $\varepsilon_{*} \underline{\mathcal{G}}$ is bounded by $\left(\hat{Z}^{\prime}\right)^{-1}$.

Let $\underline{\mathbb{G}}_{0} \cong\left(\left(L^{+} G\right)_{\mathbb{F}}, b \sigma^{*}\right)$ be a local $G$-shtuka over $\mathbb{F}$ with $b \in L G(\mathbb{F})$. Then $\underline{\mathbb{G}}_{0}^{\prime}:=\varepsilon_{*} \underline{\mathbb{G}}_{0} \cong$ $\left(\left(L^{+} G^{\prime}\right)_{\mathbb{F}}, b^{\prime} \sigma^{*}\right)$ with $b^{\prime}=\varepsilon(b)$. Kottwitz's classification of isogeny classes is functorial in the sense that $\nu_{b^{\prime}}=\varepsilon \circ \nu_{b}$ and $\varepsilon \circ \kappa_{G}=\kappa_{G^{\prime}} \circ \varepsilon$ for the induced $\Gamma$-equivariant morphism $\varepsilon: \pi_{1}(G) \rightarrow \pi_{1}\left(G^{\prime}\right)$. We also obtain a morphism of Rapoport-Zink spaces

$$
\varepsilon_{*}: \breve{\mathcal{M}}_{\underline{\mathbb{G}}_{0}}^{\hat{Z}^{-1}} \longrightarrow \breve{\mathcal{M}}_{\underline{\mathbb{G}}_{0}^{\prime}}^{\hat{Z}^{\prime-1}},(\underline{\mathcal{G}}, \bar{\delta}) \longmapsto\left(\varepsilon_{*} \underline{\mathcal{G}}, \varepsilon_{*} \bar{\delta}\right)
$$

which is equivariant for the group $J_{b}^{G}$ that acts on the target via the morphism of algebraic groups over $\mathbb{F}_{q}((z))$,

$$
J_{b}^{G} \longrightarrow J_{b^{\prime}}^{G^{\prime}}, \quad g \longmapsto \varepsilon(g)
$$

\section{Period spaces for bounded local $G$-shtukas}

In this section we construct period spaces. These will be strictly $\mathbb{F}_{q}((\zeta))$-analytic spaces in the sense of Berkovich [7], [8]. We equip the category of $\mathbb{F}_{q}((\zeta))$-schemes and the category of $\mathbb{F}_{q}((\zeta))$-analytic spaces with the étale topology; see $[8, \S 4.1]$. Recall the group scheme $G \times_{\mathbb{F}_{q} \llbracket z \rrbracket} \operatorname{Spec} \mathbb{F}_{q}((\zeta)) \llbracket z-\zeta \rrbracket$, which is reductive because $\mathbb{F}_{q} \llbracket z \rrbracket \rightarrow \mathbb{F}_{q}((\zeta)) \llbracket z-\zeta \rrbracket, z \mapsto z=$ $\zeta+(z-\zeta)$ factors through $\mathbb{F}_{q}((z))$, and recall its affine Grassmannian $\operatorname{Gr}_{G}^{\mathbf{B}_{\mathrm{dR}}}$ from (1.2). For $G=\mathrm{GL}_{r}$, Hilbert 90 for loop groups [56, Proposition 2.3] shows that

$$
\operatorname{Gr}_{\mathrm{GL}_{r}}^{\mathrm{B}_{\mathrm{dR}}}(L)=\mathrm{GL}_{r}(L((z-\zeta))) / \mathrm{GL}_{r}(L \llbracket z-\zeta \rrbracket)
$$

for any field extension $L / \mathbb{F}_{q}((\zeta))$.

Again, for all $G$ as above, the morphism of sheaves of sets on $\operatorname{Spec} \mathbb{F}_{q}((\zeta))$,

$$
G\left(\mathcal{O}_{X}((z-\zeta))\right) \rightarrow \operatorname{Gr}_{G}^{\mathbf{B}_{\mathrm{dR}}}(X),
$$

admits local sections for the étale topology. By [93, Proposition 13.1.1] there is a finite separable field extension $L_{0} \supset \mathbb{F}_{q}((\zeta))$ such that $G_{L_{0}}:=G \otimes_{\mathbb{F}_{q} \llbracket z \rrbracket, z \mapsto \zeta} L_{0}$ splits. Therefore, the group $G \otimes_{\mathbb{F}_{q} \llbracket z \rrbracket} \mathbb{F}_{q}((\zeta))((z-\zeta))$ over $\mathbb{F}_{q}((\zeta))((z-\zeta))$ is unramified. Thus, the inertia group of $\operatorname{Gal}\left(\mathbb{F}_{q}((\zeta))((z-\zeta))^{\mathrm{sep}} / \mathbb{F}_{q}((\zeta))((z-\zeta))\right)$ acts trivially on $\pi_{1}(G)$ and the connected components of $\operatorname{Gr}_{G}^{\mathbf{B}_{\mathrm{dR}}} \widehat{\otimes}_{\mathbb{F}_{q}((\zeta))} \mathbb{F}_{q}((\zeta))^{\text {alg }}$ are in canonical bijection with $\pi_{1}(G)$ by [77, Theorem 5.1]. For every field extension $L \subset \mathbb{F}_{q}((\zeta))^{\text {alg }}$ of $\mathbb{F}_{q}((\zeta))$ we then obtain from [76, Lemma 2.2.6] that

$$
\pi_{0}\left(\operatorname{Gr}_{G}^{\mathbf{B}_{\mathrm{dR}}} \widehat{\otimes}_{\mathbb{F}_{q}((z))} L\right) \cong \pi_{1}(G) / \operatorname{Gal}\left(L^{\mathrm{sep}} / L\right),
$$

where the quotient is the set of $\operatorname{Gal}\left(L^{\mathrm{sep}} / L\right)$-orbits. It has a natural projection to the group of coinvariants $\pi_{1}(G)_{\Gamma_{L}}$. In particular, $\pi_{0}\left(\mathrm{Gr}_{G}^{\mathrm{B}_{\mathrm{dR}}} \widehat{\otimes}_{\mathbb{F}_{q}((z))} L_{0}\right)=\pi_{1}(G)$.

Definition 4.1. Let $X$ be an $\mathbb{F}_{q}((\zeta))$-scheme or a strictly $\mathbb{F}_{q}((\zeta))$-analytic space. A HodgePink G-structure over $X$ is an element $\gamma$ in $\operatorname{Gr}_{G}^{\mathbf{B}_{\mathrm{dr}}}(X)$. For a Hodge-Pink $G$-structure 
$\gamma \in \operatorname{Gr}_{G}^{\mathbf{B}_{\mathrm{dR}}}(L)$ with values in a field $L$, we let $\gamma^{\#} \in \pi_{1}(G)_{\Gamma}$ be its image under the projection $\pi_{0}\left(\operatorname{Gr}_{G}^{B_{\mathrm{dR}}}\right) \rightarrow \pi_{1}(G)_{\Gamma}$ induced by (4.1).

A Hodge-Pink structure of rank $r$ over $X$ is a sheaf $\mathfrak{q}$ on $X$ of $\mathcal{O}_{X} \llbracket z-\zeta \rrbracket$-submodules of $\mathcal{O}_{X}((z-\zeta))^{r}$ that is finitely generated as $\mathcal{O}_{X} \llbracket z-\zeta \rrbracket$-module, is a direct summand as $\mathcal{O}_{X}$-module and satisfies $\mathcal{O}_{X}((z-\zeta)) \cdot \mathfrak{q}=\mathcal{O}_{X}((z-\zeta))^{r}$.

Remark 4.2. By [87, Proposition 2.2.5], a Hodge-Pink structure $\mathfrak{q}$ of rank $r$ over $X$ is Zariski-locally on $X$ free of rank $r$ as $\mathcal{O}_{X} \llbracket z-\zeta \rrbracket$-module. In particular, it is of the form $\mathfrak{q}=\gamma \cdot \mathcal{O}_{X} \llbracket z-\zeta \rrbracket^{r} \subset \mathcal{O}_{X}((z-\zeta))^{r}$ for a uniquely determined Hodge-Pink GL $_{r}$-structure $\gamma \in \operatorname{Gr}_{\mathrm{GL}_{r}}^{\mathbf{B}_{\mathrm{dR}}}(X)$ over $X$. This yields an equivalence between Hodge-Pink $\mathrm{GL}_{r}$-structures and Hodge-Pink structures of rank $r$ over $X$.

To define the notion of weak admissibility, recall from [54, Definitions 3.5.1 and 3.5.2] that a $z$-isocrystal over $\mathbb{F}$ is a pair $\left(D, \tau_{D}\right)$ consisting of a finite-dimensional $\mathbb{F}((z))$-vector space $D$ and an $\mathbb{F}((z))$-isomorphism $\tau_{D}: \sigma^{*} D \stackrel{\sim}{\longrightarrow}$. A Hodge-Pink structure on $\left(D, \tau_{D}\right)$ over a field extension $L$ of $\mathbb{F}((\zeta))$ is a free $L \llbracket z-\zeta \rrbracket$-submodule $\mathfrak{q}_{D} \subset D \otimes_{\mathbb{F}((z))} L((z-\zeta))$ of full rank. Here, as always, we use the homomorphism $\mathbb{F}((z)) \hookrightarrow \mathbb{F}((\zeta)) \llbracket z-\zeta \rrbracket, z \mapsto z=\zeta+(z-\zeta)$.

Definition 4.3. Assume that $\mathbb{F}((\zeta)) \subset L$ and let $b \in L G(\mathbb{F})$ and $\gamma \in \operatorname{Gr}_{G}^{\mathbf{B}_{\mathrm{dR}}}(L)$.

(a) Let $\rho: G_{\mathbb{F}_{q}((z))} \rightarrow \mathrm{GL}_{r, \mathbb{F}_{q}((z))}$ be in $\operatorname{Rep}_{\mathbb{F}_{q}((z))} G$ and set $V=\mathbb{F}_{q}((z)) \oplus r$, the representation space. We consider the elements $\rho(b) \in \mathrm{GL}_{r}(\mathbb{F}((z)))$ and $\rho(\gamma) \in \operatorname{Gr}_{\mathrm{GL}_{r}}^{\mathbf{B}_{\mathrm{dR}}}(L)=$ $\mathrm{GL}_{r}(L((z-\zeta))) / \mathrm{GL}_{r}(L \llbracket z-\zeta \rrbracket)$. Then we define the $z$-isocrystal

$$
\underline{D}_{b}(V, \rho):=\left(D, \tau_{D}\right):=\left(V \otimes_{\mathbb{F}_{q}((z))} \mathbb{F}((z)), \rho\left(\sigma^{*} b\right) \sigma^{*}\right)
$$

over $\mathbb{F}$ and the Hodge-Pink structure

$$
\mathfrak{q}_{D}(V):=\rho(\gamma) \cdot V \otimes_{\mathbb{F}_{q}((z))} L \llbracket z-\zeta \rrbracket \subset V \otimes_{\mathbb{F}_{q}((z))} L((z-\zeta))=D \otimes_{\mathbb{F}((z))} L((z-\zeta))
$$

on it over $L$. We set $\underline{D}_{b, \gamma}(V):=\left(V \otimes_{\mathbb{F}_{q}((z))} \mathbb{F}((z)), \rho\left(\sigma^{*} b\right) \sigma^{*}, \mathfrak{q}_{D}(V)\right)$.

(b) Let $\underline{D}=\left(D, \tau_{D}, \mathfrak{q}_{D}\right)$ be a $z$-isocrystal over $\mathbb{F}$ with Hodge-Pink structure over $L$ and let $\operatorname{det} \tau_{D}$ be the determinant of the matrix representing $\tau_{D}$ with respect to an $\mathbb{F}((z))$-basis of $D$. The $z$-adic valuation $t_{N}(\underline{D}):=\operatorname{ord}_{z}\left(\operatorname{det} \tau_{D}\right)$ is independent of this basis and is called the Newton degree of $\underline{D}$. The integer $t_{H}(\underline{D})$ with $\wedge^{r} \mathfrak{q}_{D}=$ $(z-\zeta)^{-t_{H}(\underline{D})} \wedge^{r} \mathfrak{p}_{D}$ is called the Hodge degree of $\underline{D}$, where $\mathfrak{p}_{D}:=D \otimes_{\mathbb{F}((z))} L \llbracket z-\zeta \rrbracket$.

In particular, we have $t_{N}\left(\underline{D}_{b, \gamma}(V)\right)=\operatorname{ord}_{z}\left(\operatorname{det} \rho\left(\sigma^{*} b\right)\right)=\operatorname{ord}_{z}(\operatorname{det} \rho(b))$ and $t_{H}\left(\underline{D}_{b, \gamma}(V)\right)=-\operatorname{ord}_{z-\zeta}(\operatorname{det} \rho(\gamma))$.

(c) We say that $\underline{D}$ is weakly admissible if $t_{H}(\underline{D})=t_{N}(\underline{D})$ and the following equivalent conditions are satisfied (compare [50, Definition 2.2.4]):

- $t_{H}\left(\underline{D}^{\prime}\right) \leq t_{N}\left(\underline{D}^{\prime}\right)$ for every strict subobject

$$
\underline{D}^{\prime}=\left(D^{\prime},\left.\tau_{D}\right|_{\sigma^{*} D^{\prime}}, \mathfrak{q}_{D} \cap D^{\prime} \otimes_{\mathbb{F}((z))} L((z-\zeta))\right)
$$

of $\underline{D}$, where $D^{\prime} \subset D$ is a $\tau_{D^{-}}$-stable $\mathbb{F}((z))$-subspace,

- $t_{H}\left(\underline{D}^{\prime \prime}\right) \geq t_{N}\left(\underline{D}^{\prime \prime}\right)$ for every strict quotient object $\underline{D}^{\prime \prime}=\left(D^{\prime \prime}, \tau_{D^{\prime \prime}}, \mathfrak{q}_{D^{\prime \prime}}\right)$ of $\underline{D}$, where $f: D \rightarrow D^{\prime \prime}$ is a $\tau_{D^{-}}$-stable $\mathbb{F}((z))$-quotient space and $\mathfrak{q}_{D^{\prime \prime}}=(f \otimes \mathrm{id})\left(\mathfrak{q}_{D}\right)$. 
Remark 4.4. (a) The Hodge-Pink structure $\mathfrak{q}_{D}$ on $\left(D, \tau_{D}\right)$ induces a decreasing HodgePink filtration on $D_{L}:=D \otimes_{\mathbb{F}((z)), z \mapsto \zeta} L=\mathfrak{p}_{D} /(z-\zeta) \mathfrak{p}_{D}$ given by

$$
F i l^{i} D_{L}:=\left((z-\zeta)^{i} \mathfrak{q}_{D} \cap \mathfrak{p}_{D}\right) /\left((z-\zeta)^{i} \mathfrak{q}_{D} \cap(z-\zeta) \mathfrak{p}_{D}\right)=\operatorname{im}\left((z-\zeta)^{i} \mathfrak{q}_{D} \cap \mathfrak{p}_{D} \rightarrow D_{L}\right) .
$$

If $\left(D, \tau_{D}, F i l^{\bullet} D_{L}\right)$ is weakly admissible in the sense of Fontaine (see [38, Définition 4.1.4]), then $\left(D, \tau_{D}, \mathfrak{q}_{D}\right)$ is weakly admissible, but the converse is false in general. An example is $D=\mathbb{F}((z))^{2}, \tau_{D}=z^{-1}, \mathfrak{q}_{D}=\left(\begin{array}{c}z-\zeta \\ 1\end{array}\right) L \llbracket z-\zeta \rrbracket+(z-\zeta)^{2} \mathfrak{p}_{D}$ and $D^{\prime}=\left(\begin{array}{l}0 \\ 1\end{array}\right) \mathbb{F}((z))$. This is due to the fact that our Hodge slope $t_{H}\left(D, \tau_{D}, \mathfrak{q}_{D}\right)$ equals Fontaine's Hodge slope $t_{H}\left(D, \tau_{D}, F i l^{\bullet} D_{L}\right):=\sum_{i \in \mathbb{Z}} i \cdot \operatorname{dim}_{L} F i l^{i} D_{L} / F i l^{i+1} D_{L}$, see [50, p. 1290 before Definition 2.2.4] and that the subspace $F i l^{i} D_{L}^{\prime}$ of $D_{L}^{\prime}$ induced by $\underline{D}^{\prime}$ from (4.2) is (in general strictly) contained in $D_{L}^{\prime} \cap F i l^{i} D_{L}$.

(b) We obtain an $\mathbb{F}_{q}((z))$-linear tensor functor $\underline{D}_{b, \gamma}: V \mapsto \underline{D}_{b, \gamma}(V)$. Namely, if $f:(V, \rho) \rightarrow\left(V^{\prime}, \rho^{\prime}\right)$ is a morphism in $\operatorname{Rep}_{\mathbb{F}_{q}((z))} G$ and $\left(D, \tau_{D}, \mathfrak{q}_{D}\right)=\underline{D}_{b, \gamma}(V)$ and $\left(D^{\prime}, \tau_{D^{\prime}}, \mathfrak{q}_{D^{\prime}}\right)=\underline{D}_{b, \gamma}\left(V^{\prime}\right)$, then $\sigma^{*} f=f$ and so $f \circ \tau_{D}=\left(f \circ \rho\left(\sigma^{*} b\right)\right) \sigma^{*}=\left(\rho^{\prime}\left(\sigma^{*} b\right) \circ f\right) \sigma^{*}=$ $\left(\rho^{\prime}\left(\sigma^{*} b\right) \circ \sigma^{*} f\right) \sigma^{*}=\tau_{D^{\prime}} \circ \sigma^{*} f$ and $f\left(\mathfrak{q}_{D}\right) \subset \mathfrak{q}_{D^{\prime}}$. Furthermore, the compatibility with tensor products

$$
\left(D, \tau_{D}, \mathfrak{q}_{D}\right) \otimes\left(D^{\prime}, \tau_{D^{\prime}}, \mathfrak{q}_{D^{\prime}}\right):=\left(D \otimes_{\mathbb{F}((z))} D^{\prime}, \tau_{D} \otimes \tau_{D^{\prime}}, \mathfrak{q}_{D} \otimes_{L \llbracket z-\zeta \rrbracket} \mathfrak{q}_{D^{\prime}}\right)
$$

is clear.

If $L$ is a finite field extension of $\mathbb{F}((\zeta))$, then 'weakly admissible implies admissible' by the analogue [50, Theorem 2.5.3] of the theorem of Colmez and Fontaine [28, Théorème A]. More precisely, when $\underline{D}_{b, \gamma}(V)$ is weakly admissible, it is even admissible; that is, it arises from a local shtuka over $\mathcal{O}_{L}$ via the analogue $\mathbb{H}$ of Fontaine's mysterious functor; see [50, $\S 2.3]$ or Theorem 8.1. In contrast, if $L$ is algebraically closed, weakly admissible does not imply admissible. In general, there is a criterion for admissibility in terms of $\sigma$-bundles over the analogue of the Robba ring as follows.

We consider field extensions $L$ of $\mathbb{F}_{q}((\zeta))$ equipped with an absolute value $||:. L \rightarrow \mathbb{R}_{\geq 0}$ extending the $\zeta$-adic absolute value on $\mathbb{F}_{q}((\zeta))$ such that $L$ is complete with respect to $|$.$| .$ We call such an $L$ a complete valued field extension of $\mathbb{F}_{q}((\zeta))$, and we let $\bar{L}:=\widehat{L^{\mathrm{alg}}}$ be the completion of an algebraic closure of $L$. For a rational number $s>0$, we define the ring

$$
L\left\langle\frac{z}{\zeta^{s}}, z^{-1}\right\}:=\left\{\sum_{i=-\infty}^{\infty} b_{i} z^{i}: b_{i} \in L,\left|b_{i}\right||\zeta|^{s^{\prime} i} \rightarrow 0(i \rightarrow \pm \infty) \text { for all } s^{\prime} \geq s\right\} .
$$

It equals the ring of rigid analytic functions on the punctured $\operatorname{disc}\left\{0<|z| \leq|\zeta|^{s}\right\}$ over $L$ of radius $|\zeta|^{s}$. The ring $L\left\langle\frac{z}{\zeta^{s}}, z^{-1}\right\}$ is the function field analogue of the Robba ring; see $[49, \S 2.8]$. It contains the element

$$
t_{-}:=\prod_{i \in \mathbb{N}_{0}}\left(1-\frac{\zeta^{q^{i}}}{z}\right), \quad \text { which satisfies } \quad t_{-}=\left(1-\frac{\zeta}{z}\right) \cdot \sigma^{*}\left(t_{-}\right) .
$$

Definition 4.5. Let $s \in \mathbb{Q}$ satisfy $1>s>\frac{1}{q}$. A $\sigma$-bundle (on $\left\{0<|z| \leq|\zeta|^{s}\right\}$ ) over $L$ is a pair $\underline{\mathcal{F}}=\left(\mathcal{F}, \tau_{\mathcal{F}}\right)$ consisting of a locally free $L\left\langle\frac{z}{\zeta^{s}}, z^{-1}\right\}$-module $\mathcal{F}$ of finite rank together with an isomorphism $\tau_{\mathcal{F}}: \sigma^{*} \mathcal{F} \stackrel{\sim}{\longrightarrow} \iota^{*} \mathcal{F}$ of $L\left\langle\frac{z}{\zeta^{q s}}, z^{-1}\right\}$-modules, where 
$\sigma^{*} \mathcal{F}:=\mathcal{F} \otimes_{L\left\langle\frac{z}{\zeta^{s}}, z^{-1}\right\}, \sigma} L\left\langle\frac{z}{\zeta^{q s}}, z^{-1}\right\}$ and $\iota^{*} \mathcal{F}:=\mathcal{F} \otimes_{L\left\langle\frac{z}{\zeta^{s}}, z^{-1}\right\}, \iota} L\left\langle\frac{z}{\zeta^{q s}}, z^{-1}\right\}$ for the natural inclusion $\iota: L\left\langle\frac{z}{\zeta^{s}}, z^{-1}\right\} \hookrightarrow L\left\langle\frac{z}{\zeta^{q s}}, z^{-1}\right\}, \sum_{i} b_{i} z^{i} \mapsto \sum_{i} b_{i} z^{i}$. The abelian group $\operatorname{Hom}_{\sigma}\left(\underline{\mathcal{F}}, \underline{\mathcal{F}}^{\prime}\right)$ of morphisms between two $\sigma$-bundles $\underline{\mathcal{F}}=\left(\mathcal{F}, \tau_{\mathcal{F}}\right)$ and $\underline{\mathcal{F}}^{\prime}=\left(\mathcal{F}^{\prime}, \tau_{\mathcal{F}^{\prime}}\right)$ consists of all $L\left\langle\frac{z}{\zeta^{s}}, z^{-1}\right\}$-homomorphisms $f: \mathcal{F} \rightarrow \mathcal{F}^{\prime}$ that satisfy $\tau_{\mathcal{F}^{\prime}} \circ \sigma^{*} f=\iota^{*} f \circ \tau_{\mathcal{F}}$.

The category of $\sigma$-bundles over $L$ is an $\mathbb{F}_{q}((z))$-linear rigid additive tensor category with unit object $\underline{\mathcal{O}}(0):=\left(L\left\langle\frac{z}{\zeta^{s}}, z^{-1}\right\}, \tau_{\mathcal{O}(0)}=\mathrm{id}\right)$.

Example 4.6. For $d \in \mathbb{Z}$ we define the $\sigma$-bundle $\underline{\mathcal{O}}(d)$ over $L$ as the pair $\left(L\left\langle\frac{z}{\zeta^{s}}, z^{-1}\right\}, \tau_{\mathcal{O}(d)}=z^{-d}\right.$. id). For more examples, let $d$ and $n$ be relatively prime integers with $n>0$. Consider the matrix

$$
A_{d, n}:=\left(\begin{array}{cccccc}
0 & \ldots & \ldots & 0 & z^{-d} \\
1 & \ddots & & & & 0 \\
0 & \ddots & \ddots & \ddots & \\
& \ddots & \ddots & \ddots & \\
0 & \cdots & 0 & 1 & 0
\end{array}\right) \in \operatorname{GL}_{n}\left(L\left\langle\frac{z}{\zeta^{s}}, z^{-1}\right\}\right)
$$

Let $\underline{\mathcal{F}}_{d, n}=\left(L\left\langle\frac{z}{\zeta^{s}}, z^{-1}\right\}^{n}, \tau_{\mathcal{F}_{d, n}}=A_{d, n}\right)$. It is a $\sigma$-bundle of rank $n$ over $L$. As a special case if $n=1$, we obtain $\underline{\mathcal{F}}_{d, 1}=\underline{\mathcal{O}}(d)$.

Proposition 4.7 ([55, Theorem 11.1 and Corollary 11.8].). If L is algebraically closed (and complete), every $\sigma$-bundle $\underline{\mathcal{F}}$ is isomorphic to a direct sum $\bigoplus_{i} \underline{\mathcal{F}}_{d_{i}, n_{i}}$ for uniquely determined pairs $\left(d_{i}, n_{i}\right)$ up to permutation with $\operatorname{gcd}\left(d_{i}, n_{i}\right)=1$. One has $\wedge^{n} \underline{\mathcal{F}} \cong \underline{\mathcal{F}}_{d, 1}=$ $\underline{\mathcal{O}}(d)$ where $n=\operatorname{rk} \underline{\mathcal{F}}=\sum_{i} n_{i}$ and $d=\sum_{i} d_{i}$. One calls $d$ the degree of $\underline{\mathcal{F}}$.

Proposition 4.8 ([55, Proposition 8.5].). If $L$ is algebraically closed (and complete), then $\operatorname{Hom}_{\sigma}\left(\underline{\mathcal{F}}_{d, n}, \underline{\mathcal{F}}_{d^{\prime}, n^{\prime}}\right) \neq(0)$ if and only if $d / n \leq d^{\prime} / n^{\prime}$.

Let $\underline{D}=\left(D, \tau_{D}, \mathfrak{q}_{D}\right)$ be a $z$-isocrystal over $\mathbb{F}$ with a Hodge-Pink structure over a complete valued field extension $L$ of $\mathbb{F}((\zeta))$. To define the $\sigma$-bundles associated with $\underline{D}$, we first define the $\sigma$-bundle

$$
\mathcal{E}:=\mathcal{E}(\underline{D}):=D \otimes_{\mathbb{F}((z))} L\left\langle\frac{z}{\zeta^{s}}, z^{-1}\right\}, \quad \tau_{\mathcal{E}}:=\tau_{D} \otimes \mathrm{id}, \quad \underline{\mathcal{E}}(\underline{D})=\left(\mathcal{E}, \tau_{\mathcal{E}}\right)
$$

over $L$. Then $\mathcal{E}(\underline{D}) \otimes L \llbracket z-\zeta \rrbracket=\mathfrak{p}_{D}:=D \otimes_{\mathbb{F}((z))} L \llbracket z-\zeta \rrbracket$ and the Hodge-Pink structure $\mathfrak{q}_{D} \subset \mathcal{E} \otimes L \llbracket z-\zeta \rrbracket\left[\frac{1}{z-\zeta}\right]$ defines a $\sigma$-bundle $\underline{\mathcal{F}}(\underline{D})$ over $L$ that is a modification of $\underline{\mathcal{E}}(\underline{D})$ at $z=\zeta^{q^{i}}$ for $i \in \mathbb{N}_{0}$ as follows. Consider the isomorphism $\eta_{i}:=\left(\tau_{\mathcal{E}} \circ \ldots \circ\left(\sigma^{i-1}\right)^{*} \tau_{\mathcal{E}}\right) \otimes$ id

$$
\eta_{i}:\left(\sigma^{i}\right)^{*}\left(\mathfrak{p}_{D}\left[\frac{1}{z-\zeta}\right]\right)=\left(\left(\sigma^{i}\right)^{*} \mathcal{E}\right) \otimes L \llbracket z-\zeta^{q^{i}} \rrbracket\left[\frac{1}{z-\zeta^{i}}\right] \stackrel{\sim}{\longrightarrow} \mathcal{E} \otimes L \llbracket z-\zeta^{q^{i}} \rrbracket\left[\frac{1}{z-\zeta^{i}}\right] .
$$

We define $\mathcal{F}=\mathcal{F}(\underline{D})$ as the $L\left\langle\frac{z}{\zeta^{s}}, z^{-1}\right\}$-submodule of $\mathcal{E}\left[\frac{1}{t_{-}}\right]$that coincides with $\mathcal{E}$ outside $z=\zeta^{q^{i}}$ for $i \in \mathbb{N}_{0}$ and at $z=\zeta^{q^{i}}$ satisfies $\mathcal{F} \otimes L \llbracket z-\zeta^{q^{i}} \rrbracket=\eta_{i}\left(\sigma^{i *} \mathfrak{q}_{D}\right)$; that is,

$$
\mathcal{F}:=\left\{m \in \mathcal{E}\left[\frac{1}{t_{-}}\right]: \eta_{i}^{-1}(m) \in \sigma^{i *} \mathfrak{q}_{D} \text { for } i \in \mathbb{N}_{0}\right\}
$$

This can equivalently be viewed as the global sections over $\left\{0<|z| \leq|\zeta|^{s}\right\}$ of the sheaf $\widetilde{\mathcal{F}}$ obtained as the modification of the sheaf associated with $\mathcal{E}$ at the discrete set $\left\{z=\zeta^{q^{i}}\right.$ : $\left.i \in \mathbb{N}_{0}\right\}$ according to the rule given in (4.4). 
By construction, $\tau_{\mathcal{E}}$ induces on $\mathcal{F}$ the structure of a $\sigma$-bundle $\underline{\mathcal{F}}(\underline{D})=\left(\mathcal{F}, \tau_{\mathcal{F}}\right)$ over $L$, and $\underline{\mathcal{F}}(\underline{D})$ is the unique $\sigma$-subbundle of $\underline{\mathcal{E}}\left[\frac{1}{t_{-}}\right]$that coincides with $\underline{\mathcal{E}}$ outside $z=\zeta^{q^{i}}$ for $i \in \mathbb{N}_{0}$ and satisfies $\mathcal{F} \otimes L \llbracket z-\zeta \rrbracket=\mathfrak{q}_{D}$. This characterisation implies that the assignment $\underline{D} \mapsto(\underline{\mathcal{E}}(\underline{D}), \underline{\mathcal{F}}(\underline{D}))$ is an $\mathbb{F}_{q}((z))$-linear tensor functor.

Definition 4.9. The pair of $\sigma$-bundles associated with $\underline{D}$ is the pair $(\underline{\mathcal{E}}(\underline{D}), \underline{\mathcal{F}}(\underline{D}))$ constructed above.

The $z$-isocrystal with Hodge-Pink structure $\underline{D}$ is said to be admissible if $\underline{\mathcal{F}}(\underline{D}) \otimes_{L\left\langle\frac{z}{\zeta^{s}}, z^{-1}\right\}} \bar{L}\left\langle\frac{z}{\zeta^{s}}, z^{-1}\right\} \cong\left(\underline{\mathcal{F}}_{0,1}\right)^{\oplus \operatorname{dim} D} \otimes_{L\left\langle\frac{z}{\zeta^{s}}, z^{-1}\right\}} \bar{L}\left\langle\frac{z}{\zeta^{s}}, z^{-1}\right\}$.

In the notation of Definition 4.3, let $\underline{D}=\underline{D}_{b, \gamma}(V)$ for a representation $\rho: G \rightarrow \operatorname{GL}(V)$ in $\operatorname{Rep}_{\mathbb{F}_{q}((z))} G$ and write $\left(\underline{\mathcal{E}}_{b, \gamma}(V), \underline{\mathcal{F}}_{b, \gamma}(V)\right):=(\underline{\mathcal{E}}(\underline{D}), \underline{\mathcal{F}}(\underline{D}))$.

As a motivation for this definition, note that $\underline{D}$ is admissible if and only if it arises from a local $\mathrm{GL}_{r}$-shtuka over $\mathcal{O}_{L}$ by [50, Theorem 2.4.7 and Definition 2.3.3].

Proposition 4.10 ([50, Lemma 2.4.5].). For every z-isocrystal with Hodge-Pink structure $\underline{D}$ over $L$, the degree (defined in Proposition 4.7) satisfies $\operatorname{deg} \underline{\mathcal{F}}(\underline{D})=t_{H}(\underline{D})-t_{N}(\underline{D})$ and $\operatorname{deg} \underline{\mathcal{E}}(\underline{D})=-t_{N}(\underline{D})$.

Corollary 4.11. If $\underline{D}_{b, \gamma}(V)$ is admissible, then $\underline{D}_{b, \gamma}(V)$ is weakly admissible.

Proof. If $\underline{D}:=\underline{D}_{b, \gamma}(V)$ is admissible, then $\underline{\mathcal{F}}(\underline{D}) \otimes_{L\left\langle\frac{z}{\zeta^{s}}, z^{-1}\right\}} \bar{L}\left\langle\frac{z}{\zeta^{s}}, z^{-1}\right\} \cong\left(\underline{\mathcal{F}}_{0,1}\right)^{\oplus \operatorname{dim} V}$ and therefore $t_{H}(\underline{D})-t_{N}(\underline{D})=\operatorname{deg}(\underline{\mathcal{F}}(\underline{D}))=0$. If $\underline{D}^{\prime} \subset \underline{D}$ is a strict subobject, then $\underline{\mathcal{F}}\left(\underline{D}^{\prime}\right) \subset \underline{\mathcal{F}}(\underline{D})$ is a $\sigma$-subbundle. It satisfies $\underline{\mathcal{F}}\left(\underline{D^{\prime}}\right) \otimes_{L\left\langle\frac{z}{\zeta^{s}}, z^{-1}\right\}} \bar{L}\left\langle\frac{z}{\zeta^{s}}, z^{-1}\right\} \cong \bigoplus_{i} \underline{\mathcal{F}}_{d_{i}, n_{i}}$ for some $d_{i}, n_{i}$ by Proposition 4.7. By Proposition 4.8, all $d_{i} \leq 0$ and hence $t_{H}\left(\underline{D}^{\prime}\right)-t_{N}\left(\underline{D}^{\prime}\right)=$ $\operatorname{deg} \underline{\mathcal{F}}\left(\underline{D}^{\prime}\right)=\sum_{i} d_{i} \leq 0$.

Lemma 4.12. Let $\nu_{b}: \mathbb{D}_{\mathbb{F}((z))} \rightarrow G_{\mathbb{F}((z))}$ be the Newton point associated with $b$; see Remark 3.6(a). Let $\rho: G \rightarrow \mathrm{GL}(V)$ be a representation in $\operatorname{Rep}_{\mathbb{F}_{q}((z))} G$. Then under the canonical identifications $\pi_{1}(\mathrm{GL}(V))_{\Gamma}=\pi_{1}(\mathrm{GL}(V))=\mathbb{Z}$ and $\operatorname{Hom}\left(\mathbb{D}_{\mathbb{F}((z))}, \mathbb{G}_{m}\right)=\mathbb{Q}$, we have

$$
\rho_{*}\left(\gamma^{\#}\right)=t_{H}\left(\underline{D}_{b, \gamma}(V)\right) \quad \text { and } \quad \operatorname{det}_{V} \circ \rho \circ \nu_{b}=t_{N}\left(\underline{D}_{b, \gamma}(V)\right) .
$$

In particular, the images $[b]^{\#}$ and $\gamma^{\#}$ of $\nu_{b}$ and $\gamma$ in $\pi_{1}(G)_{\Gamma, \mathbb{Q}}:=\pi_{1}(G)_{\Gamma} \otimes_{\mathbb{Z}} \mathbb{Q}$ coincide if and only if $t_{N}\left(\underline{D}_{b, \gamma}(V)\right)=t_{H}\left(\underline{D}_{b, \gamma}(V)\right)$ for all $V \in \operatorname{Rep}_{\mathbb{F}_{q}((z))} G$.

Proof. Because $\sigma^{*} b=b\left(\sigma^{*} b\right) \sigma^{*} b^{-1}$ - that is, $\sigma^{*} b$ and $b$ are $\sigma$-conjugate via $b$ their Newton points $\nu_{\sigma^{*} b}$ and $\nu_{b}$ are conjugate via $b$. So it suffices to show that $\operatorname{det}_{V} \circ \rho \circ \nu_{\sigma^{*} b}=t_{N}\left(\underline{D}_{b, \gamma}(V)\right)$. The latter follows from the construction of $\nu_{\sigma^{*} b}$ in [70, $\left.\S 4.2\right]$. The statement about $t_{H}$ follows from the fact that $\rho_{*}\left(\gamma^{\#}\right)=\rho(\gamma)^{\#}=-\operatorname{ord}_{z-\zeta}(\operatorname{det} \rho(\gamma))$ under the identification $\pi_{0}\left(\operatorname{Gr}_{\mathrm{GL}(V)}^{\mathbf{B}_{\mathrm{dR}}}\right)=\pi_{1}(\mathrm{GL}(V))_{\Gamma} \cong \mathbb{Z}$. If $[b]^{\#}=\gamma^{\#}$ holds in $\pi_{1}(G)_{\Gamma, \mathbb{Q}}$, then $\rho_{*}\left([b]^{\#}\right)=\rho_{*}\left(\gamma^{\#}\right)$ in $\pi_{1}(\mathrm{GL}(V))_{\Gamma, \mathbb{Q}} \cong \mathbb{Q}$. Under the last isomorphism we have $\rho_{*}\left([b]^{\#}\right)=\left(\rho \circ \nu_{b}\right)^{\#}=\operatorname{det}_{V} \circ \rho \circ \nu_{b}$. This proves one direction of the last assertion. For the other direction we use the isomorphism $\pi_{1}(G)_{\Gamma, \mathbb{Q}} \cong \pi_{1}\left(G_{\mathrm{ab}}\right)_{\Gamma, \mathbb{Q}} \cong X_{*}\left(G_{\mathrm{ab}}\right)_{\Gamma, \mathbb{Q}}$, where $G_{\mathrm{ab}}$ 
denotes the maximal abelian quotient of $G$ (compare [78, Theorem 1.15(ii)]). Now assume that $[b]^{\#} \neq \gamma^{\#}$. Then there is a homomorphism $\varphi: \pi_{1}(G)_{\Gamma, \mathbb{Q}} \rightarrow \mathbb{Q}$ of $\mathbb{Q}$-vector spaces such that $\varphi\left([b]^{\#}\right) \neq \varphi\left(\gamma^{\#}\right)$. We have $\operatorname{Hom}\left(\pi_{1}(G)_{\Gamma, \mathbb{Q}}, \mathbb{Q}\right) \cong X^{*}\left(G_{\mathrm{ab}}\right)_{\mathbb{Q}}^{\Gamma}$; thus, a nonzero integral multiple of $\varphi$ induces a morphism $\rho: G \rightarrow G_{\mathrm{ab}} \rightarrow \mathbb{G}_{m}$ over $\mathbb{F}_{q}((z))^{\text {sep }}$ that is $\Gamma$-invariant and therefore defined over $\mathbb{F}_{q}((z))$. For this representation $\rho$, we then have $t_{N}\left(\underline{D}_{b, \gamma}(V)\right) \neq t_{H}\left(\underline{D}_{b, \gamma}(V)\right)$.

Definition 4.13. We say that the pair $(b, \gamma) \in L G(\mathbb{F}) \times \operatorname{Gr}_{G}^{\mathbf{B}_{\mathrm{dR}}}(L)$ is (weakly) admissible if $[b]^{\#}=\gamma^{\#}$ in $\pi_{1}(G)_{\Gamma, \mathbb{Q}}$ and one of the following equivalent conditions holds:

(a) $\underline{D}_{b, \gamma}(V)$ is (weakly) admissible for every representation $V$ in $\operatorname{Rep}_{\mathbb{F}_{q}((z))} G$,

(b) $\underline{D}_{b, \gamma}(V)$ is (weakly) admissible for some faithful representation $V$ in $\operatorname{Rep}_{\mathbb{F}_{q}((z))} G$.

In addition, $(b, \gamma)$ is neutral if $[b]^{\#}=\gamma^{\#}$ in $\pi_{1}(G)_{\Gamma}$ already without tensoring with $\mathbb{Q}$.

Remark 4.14. If $\underline{D}_{b, \gamma}(V)$ is (weakly) admissible for every representation $V$ in $\operatorname{Rep}_{\mathbb{F}_{q}((z))} G$, then $[b]^{\#}=\gamma^{\#}$ automatically holds in $\pi_{1}(G)_{\Gamma, \mathbb{Q}}$ by Lemma 4.12.

In the analogous situation in mixed characteristic, the condition $[b]^{\#}=\gamma^{\#}$ also follows from (b), due to the fact that in that case every $W$ as in the beginning of the following proof is even a direct summand of $U$.

Proof of the equivalence in Definition 4.13. Clearly, (a) implies (b). For the converse, fix a faithful representation $V$. Then every $\mathbb{F}_{q}((z))$-rational representation $W$ of $G_{\mathbb{F}_{q}}((z))$ is a subquotient of $U:=\bigoplus_{i=1}^{r} V^{\otimes l_{i}} \otimes\left(V^{\vee}\right)^{\otimes m_{i}}$ for suitable $r, l_{i}$ and $m_{i}$. If $\underline{D}_{b, \gamma}(V)$ is weakly admissible, then this also holds for $\underline{D}_{b, \gamma}(U)$ by [50, Theorem 2.2.5]. Likewise, if $\underline{D}_{b, \gamma}(V)$ is admissible, we use the compatibility of the functor $V \mapsto \underline{\mathcal{F}}_{b, \gamma}(V)$ with direct sums, tensor products and duals to compute

$$
\begin{aligned}
& \underline{\mathcal{F}}_{b, \gamma}(U) \otimes_{L\left\langle\frac{z}{\zeta^{s}}, z^{-1}\right\}} \bar{L}\left\langle\frac{z}{\zeta^{s}}, z^{-1}\right\} \\
& \cong \bigoplus_{i=1}^{r} \underline{\mathcal{F}}_{b, \gamma}(V)^{\otimes l_{i}} \otimes\left(\underline{\mathcal{F}}_{b, \gamma}(V)^{\vee}\right)^{\otimes m_{i}} \otimes_{L\left\langle\frac{z}{\zeta^{s}}, z^{-1}\right\}} \bar{L}\left\langle\frac{z}{\zeta^{s}}, z^{-1}\right\} \\
& \cong \bigoplus_{i=1}^{r}\left(\underline{\mathcal{F}}_{0,1} \oplus \operatorname{dim} V\right)^{\otimes l_{i}} \otimes\left(\left(\underline{\mathcal{F}}_{0,1} \oplus \operatorname{dim} V\right)^{\vee}\right)^{\otimes m_{i}} \otimes_{L\left\langle\frac{z}{\zeta^{s}}, z^{-1}\right\}} \bar{L}\left\langle\frac{z}{\zeta^{s}}, z^{-1}\right\} \\
& \left.\cong \underline{\mathcal{F}}_{0,1} \oplus \operatorname{dim} U \otimes_{L\left\langle\frac{z}{\zeta^{s}}\right.}, z^{-1}\right\} \bar{L}\left\langle\frac{z}{\zeta^{s}}, z^{-1}\right\} .
\end{aligned}
$$

So if $\underline{D}_{b, \gamma}(V)$ is admissible, $\underline{D}_{b, \gamma}(U)$ also is. Therefore, it suffices to show that (weak) admissibility is preserved under passage to subrepresentations and quotient representations.

By Lemma 4.12, the condition $[b]^{\#}=\gamma^{\#}$ in $\pi_{1}(G)_{\Gamma, \mathbb{Q}}$ implies $t_{N}\left(\underline{D}_{b, \gamma}(W)\right)=$ $t_{H}\left(\underline{D}_{b, \gamma}(W)\right)$ for all representations $W$. Now let $U$ be a representation such that $\underline{D}_{b, \gamma}(U)$ is weakly admissible. Then the equivalent conditions from Definition 4.3(c) show that for every subrepresentation or quotient representation $W$ of $U$ the $z$-isocrystal with HodgePink structure $\underline{D}_{b, \gamma}(W)$ is also weakly admissible. 
If $\underline{D}_{b, \gamma}(U)$ is actually admissible, then $\underline{\mathcal{F}}_{b, \gamma}(U) \otimes_{L\left\langle\frac{z}{\zeta^{s}}, z^{-1}\right\}} \bar{L}\left\langle\frac{z}{\zeta^{s}}, z^{-1}\right\} \cong \underline{\mathcal{F}}_{0,1} \oplus \operatorname{dim} U$. If $W \subset U$ is a subrepresentation, then the $\sigma$-subbundle $\underline{\mathcal{F}}_{b, \gamma}(W) \subset \underline{\mathcal{F}}_{b, \gamma}(U)$ satisfies $\left.\underline{\mathcal{F}}_{b, \gamma}(W) \otimes_{L\left\langle\frac{z}{\zeta^{s}}\right.}, z^{-1}\right\} \bar{L}\left\langle\frac{z}{\zeta^{s}}, z^{-1}\right\} \cong \bigoplus_{i} \underline{\mathcal{F}}_{d_{i}, n_{i}}$ for some $d_{i}, n_{i}$ by Proposition 4.7. By Proposition 4.8, all $d_{i} \leq 0$. Because

$$
\sum_{i} d_{i}=\operatorname{deg} \underline{\mathcal{F}}_{b, \gamma}(W)=t_{H}\left(\underline{D}_{b, \gamma}(W)\right)-t_{N}\left(\underline{D}_{b, \gamma}(W)\right)=0
$$

by Proposition 4.10, all $d_{i}$ must be zero and $\underline{\mathcal{F}}_{b, \gamma}(W)$ is admissible. Dually, if $W$ is a quotient representation of $U$, the $\sigma$-quotient-bundle $\underline{\mathcal{F}}_{b, \gamma}(W)$ of $\underline{\mathcal{F}}_{b, \gamma}(U)$ satisfies $\underline{\mathcal{F}}_{b, \gamma}(W) \otimes_{L\left\langle\frac{z}{\zeta^{s}}, z^{-1}\right\}} \bar{L}\left\langle\frac{z}{\zeta^{s}}, z^{-1}\right\} \cong \bigoplus_{i} \underline{\mathcal{F}}_{d_{i}, n_{i}}$ for some $d_{i}, n_{i}$ with $d_{i} \geq 0$ by Proposition 4.8. Again, $\operatorname{deg} \underline{\mathcal{F}}_{b, \gamma}(W)=0$ implies $d_{i}=0$ and $\underline{\mathcal{F}}_{b, \gamma}(W)$ is admissible.

Remark 4.15. Let $b \in L G(\mathbb{F})$. Let $L_{0}$ be a finite field extension of $\mathbb{F}_{q}((\zeta))$ for which $G_{L_{0}}:=G \times_{\mathbb{F}_{q} \llbracket z \rrbracket, z \mapsto \zeta} L_{0}$ is split. Let $T$ be a maximal split torus of $G_{L_{0}}$ that contains the image of the Newton point $\nu_{b}: \mathbb{D}_{\mathbb{F}((z))} \rightarrow G_{\mathbb{F}((z))}$; see Remark 3.6(a). We may view $\nu_{b}$ as an element of $X_{*}(T)_{\mathbb{Q}}:=X_{*}(T) \otimes_{\mathbb{Z}} \mathbb{Q}$. Let $L$ be a complete valued field extension of the completion of the maximal unramified extension $\breve{L}_{0}$ of $L_{0}$ and let $\gamma \in \operatorname{Gr}_{G}^{\mathbf{B}_{\mathrm{dR}}}(L)$. By the Cartan decomposition there is a unique dominant cocharacter $\mu_{\gamma} \in X_{*}(T)$ called the Hodge point of $\gamma$ such that

$$
\gamma \in G(L \llbracket z-\zeta \rrbracket) \cdot \mu_{\gamma}(z-\zeta) \cdot G(L \llbracket z-\zeta \rrbracket) / G(L \llbracket z-\zeta \rrbracket) \subset \operatorname{Gr}_{G}^{\mathbf{B}_{\mathrm{dR}}}(L) .
$$

If $(b, \gamma)$ is weakly admissible, then $\nu_{b} \preceq \mu_{\gamma}$ (see [30, Theorem 9.5.10], which is for the arithmetic context but gives a proof that can directly be translated to our situation); in other words, $\left([b],\left\{\mu_{\gamma}\right\}\right)$ is acceptable in the sense of [79, Definition 2.5]. The converse of this is not true. However, if $\mu \in X_{*}(T)$ is dominant with $\nu_{b} \preceq \mu$, then one can show that there exists a cocharacter $\operatorname{Int}_{g} \circ \mu: \mathbb{G}_{m, L} \rightarrow G_{L}$ with $g \in G(L)$ for a finite extension $L \supset \breve{L}_{0}$, which induces a weakly admissible Hodge-Pink filtration on $\underline{D}_{b}(V)$ for all $V$. Indeed, this can be shown in the same way as the arithmetic counterpart; compare [30, Theorem 9.5.10]. Then by Remark $4.4(\mathrm{a}), \underline{D}_{b, \gamma}(V)$ is weakly admissible (and even admissible) for every HodgePink $G$-structure $\gamma \in \operatorname{Gr}_{G}^{\mathbf{B}_{\mathrm{dR}}}(L)$ that induces $\operatorname{Int}_{g} \circ \mu$, like, for example, $\gamma=g \cdot \mu(z-\zeta)$. For more details and references in the arithmetic context, compare also the discussion in [79, Section 2.2 and Proposition 3.1].

We next want to define period spaces in the bounded situation. Let $\hat{Z}=\left[\left(R, \hat{Z}_{R}\right)\right]$ be a bound as in Definition 2.2 with reflex ring $R_{\hat{Z}}=\kappa \llbracket \xi \rrbracket$ and set $E:=E_{\hat{Z}}=\kappa((\xi))$ and $\breve{E}:=$ $\mathbb{F}((\xi))$. By Proposition 2.6(d), the associated strictly $R\left[\frac{1}{\zeta}\right]$-analytic spaces $\hat{Z}_{R}^{\text {an }}$ arise by base

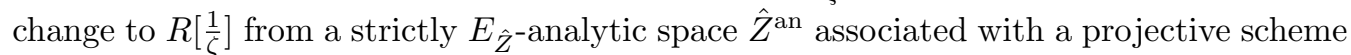
$\hat{Z}_{E}$ over $\operatorname{Spec} E_{\hat{Z}}$, which is a closed subscheme of the affine Grassmannian $\operatorname{Gr}_{G, E}^{\mathbf{B}_{\mathrm{dR}}}:=$ $\mathrm{Gr}_{G}^{\mathrm{B}_{\mathrm{dR}}} \times_{\mathbb{F}_{q}((\zeta))} \operatorname{Spec} E_{\hat{Z}}$.

Definition 4.16. We call $\mathcal{H}_{G, \hat{Z}}:=\hat{Z}_{E}$ the space of Hodge-Pink G-structures bounded by $\hat{Z}$ and set $\breve{\mathcal{H}}_{G, \hat{Z}}:=\mathcal{H}_{G, \hat{Z}} \times_{E_{\hat{Z}}}$ Spec $\breve{E}$. Let $\underline{\mathbb{G}}_{0}=\left(\left(L^{+} G\right)_{\mathbb{F}}, b \sigma^{*}\right)$ be a local $G$-shtuka over 
$\mathbb{F}$. We define the period spaces of (weakly) admissible Hodge-Pink G-structures on $\underline{\mathbb{G}}_{0}$ bounded by $\hat{Z}$ as

$$
\begin{aligned}
& \breve{\mathcal{H}}_{G, \hat{Z}, b}^{w a}:=\left\{\gamma \in \breve{\mathcal{H}}_{G, \hat{Z}}^{\text {an }}: \text { the pair }(b, \gamma) \text { is weakly admissible }\right\}, \\
& \breve{\mathcal{H}}_{G, \hat{Z}, b}^{a}:=\left\{\gamma \in \breve{\mathcal{H}}_{G, \hat{Z}}^{\text {an }}: \text { the pair }(b, \gamma) \text { is admissible }\right\} \text { and } \\
& \breve{\mathcal{H}}_{G, \hat{Z}, b}^{n a}:=\left\{\gamma \in \breve{\mathcal{H}}_{G, \hat{Z}}^{\text {an }}: \text { the pair }(b, \gamma) \text { is admissible and neutral }\right\} .
\end{aligned}
$$

$\breve{\mathcal{H}}_{G, \hat{Z}, b}^{n a}$ equals the intersection of $\breve{\mathcal{H}}_{G, \hat{Z}, b}^{a}$ with the union of those connected components of $\breve{\mathcal{H}}_{G, \hat{Z}}$ that map to $[b]^{\#} \in \pi_{1}(G)_{\Gamma}$ under the map $\pi_{0}\left(\breve{\mathcal{H}}_{G, \hat{Z}}\right) \rightarrow \pi_{0}\left(\operatorname{Gr}_{G}^{\mathbf{B}_{\mathrm{dR}}}\right) \rightarrow \pi_{1}(G)_{\Gamma}$ induced by (4.1). In particular, $\breve{\mathcal{H}}_{G, \hat{Z}, b}^{n a}$ is a union of connected components of $\breve{\mathcal{H}}_{G, \hat{Z}, b}^{a}$. The period spaces only depend on $b$ and on the generic fibres $G_{\mathbb{F}_{q}((z))}$ and $\hat{Z}_{E}$ of $G$ and $\hat{Z}$.

Remark 4.17. If $\breve{E} \subset L$, then the homomorphism $\mathbb{F}((z)) \rightarrow L \llbracket z-\zeta \rrbracket, z \mapsto z=\zeta+(z-\zeta)$ induces a homomorphism $L G(\mathbb{F})=G(\mathbb{F}((z))) \rightarrow G(L \llbracket z-\zeta \rrbracket)$. Thus, if $b^{\prime}=g b \sigma^{*}\left(g^{-1}\right)$ for some $g \in L G(\mathbb{F})$, one can check that $\gamma \mapsto \sigma^{*}(g) \cdot \gamma=: \gamma^{\prime}$ maps $\breve{\mathcal{H}}_{G, \hat{Z}, b}^{\text {wa }}$ isomorphically onto $\breve{\mathcal{H}}_{G, \hat{Z}, b^{\prime}}^{w a}$ (and likewise for $\breve{\mathcal{H}}_{G, \hat{Z}, b^{\prime}}^{a}$ and $\breve{\mathcal{H}}_{G, \hat{Z}, b^{\prime}}^{n a}$ ), because $\sigma^{*}(g)$ maps $\breve{\mathcal{H}}_{G, \hat{Z}}^{\text {an }}$ to itself by Lemma 2.10 and induces isomorphisms $\underline{D}_{b^{\prime}, \gamma^{\prime}}(V) \cong \underline{D}_{b, \gamma}(V)$ and $\underline{\mathcal{F}}_{b^{\prime}, \gamma^{\prime}}(V) \cong \underline{\mathcal{F}}_{b, \gamma}(V)$. In particular, $\breve{\mathcal{H}}_{G, \hat{Z}, b}^{w a}, \breve{\mathcal{H}}_{G, \hat{Z}, b}^{a}$ and $\breve{\mathcal{H}}_{G, \hat{Z}, b}^{n a}$ are invariant under the group $J_{b}\left(\mathbb{F}_{q}((z))\right)=$ $\operatorname{QIsog}_{\mathbb{F}}\left(\left(L^{+} G\right)_{\mathbb{F}}, b \sigma^{*}\right)$ from (3.1).

Proposition 4.18. The space $\breve{\mathcal{H}}_{G, \hat{Z}, b}^{a}$ is contained in $\breve{\mathcal{H}}_{G, \hat{Z}, b}^{w a}$ with $\breve{\mathcal{H}}_{G, \hat{Z}, b}^{a}(L)=\breve{\mathcal{H}}_{G, \hat{Z}, b}^{w a}(L)$ for all complete valued field extensions $L / \breve{E}$ satisfying the following condition: Let $\bar{L}$ be the completion of an algebraic closure of $L$, let $\bar{\ell} \subset \bar{L}$ be a subfield isomorphic to the residue field of $\bar{L}$ under the residue map $\mathcal{O}_{\bar{L}} \rightarrow \mathcal{O}_{\bar{L}} / \mathfrak{m}_{\bar{L}}$ and let $\widetilde{L}$ be the closure of the compositum $\bar{\ell} L$ inside $\bar{L}$. (In particular, if the residue field of $L$ is perfect, then $\widetilde{L}$ is the completion of the maximal unramified extension of L.) The condition is that $\widetilde{L}$ does not contain an element a with $0<|a|<1$ such that all of the q-power roots of a also lie in $\widetilde{L}$.

Proof. The inclusion $\breve{\mathcal{H}}_{G, \hat{Z}, b}^{a} \subset \breve{\mathcal{H}}_{G, \hat{Z}, b}^{w a}$ follows from Corollary 4.11. The equality $\breve{\mathcal{H}}_{G, \hat{Z}, b}^{a}(L)=\breve{\mathcal{H}}_{G, \hat{Z}, b}^{w a}(L)$ for the mentioned fields was proved in [50, Theorem 2.5.3].

Remark 4.19. (a) The condition of the proposition, and hence $\breve{\mathcal{H}}_{G, \hat{z}, b}^{a}(L)=$ $\breve{\mathcal{H}}_{G, \hat{Z}, b}^{w a}(L)$, holds if the value group of $L$ does not contain a nonzero element that is arbitrarily often divisible by $q$. This is due to the fact that the value groups of $L$ and $\widetilde{L}$ coincide. In particular, this is the case if $L$ is a finite field extension of $\breve{E}$ or, more generally, if $L$ is discretely valued or even if the value group of $L$ is finitely generated. See [50, Condition (2.3) on page 1294] for further discussion of this condition.

(b) If $L$ violates the condition - for example, if $L$ is algebraically closed and complete it can happen that $\breve{\mathcal{H}}_{G, \hat{Z}, b}^{a}(L) \subsetneq \breve{\mathcal{H}}_{G, \hat{Z}, b}^{w a}(L)$. Examples in the case $G=\mathrm{GL}_{r}$ were given in [50, Example 3.3.2]. 
Theorem 4.20. The period space $\breve{\mathcal{H}}_{G, \hat{Z}, b}^{\text {wa }}$ and the admissible locus $\breve{\mathcal{H}}_{G, \hat{Z}, b}^{a}$ are open paracompact strictly $\breve{E}$-analytic subspaces of $\breve{\mathcal{H}}_{G, \hat{Z}}^{a n}$. The intersections of any connected component of $\breve{\mathcal{H}}_{G, \hat{Z}}^{a n}$ with $\breve{\mathcal{H}}_{G, \hat{Z}, b}^{\text {wa }}$ and $\breve{\mathcal{H}}_{G, \hat{Z}, b}^{a}$ are arcwise connected. In the terminology of Remarks 2.11(b) and 4.15, the spaces $\breve{\mathcal{H}}_{G, \hat{Z}, b}^{w a}$ and $\breve{\mathcal{H}}_{G, \hat{Z}, b}^{a}$ intersect after base change to $\breve{L}_{0}$ precisely those connected components of $\operatorname{Gr}_{G, \breve{L}_{0}}^{\mathbf{B}_{d R}}$ whose image in $\pi_{1}(G)=\pi_{0}\left(\mathrm{Gr}_{G, \breve{L}_{0}}^{\mathbf{B}_{d R}}\right)$ (using (4.1)) is of the form $\mu^{\#} \in \pi_{1}(G)$ for a $\mu \in N_{\text {an }}$ with $\nu_{b} \preceq \mu$.

Proof. Choose a faithful representation $\rho: G \hookrightarrow \mathrm{GL}_{r}$ in $\operatorname{Rep}_{\mathbb{F}_{q}((z))} G$ that factors through $\mathrm{SL}_{r}$, and let $n$ be an integer as in Proposition 2.6(a) for which $\rho_{*}: \hat{Z} \rightarrow \widehat{\mathcal{F}}_{\mathrm{SL}_{r}}$ factors through $\widehat{\mathcal{F}}_{\mathrm{SL}_{r}}^{(n)}=\hat{Z}_{\mathrm{GL}_{r}, 2 n \rho^{\vee}}$; see Examples 2.8 and 2.13. By Proposition 2.6, the $\breve{E}$-analytic space $\breve{\mathcal{H}}_{G, \hat{Z}}^{\text {an }}$ is a subspace of $\mathcal{H}_{\mathrm{GL}_{r}, 2 n \rho^{\vee}}^{\text {an }} \widehat{\otimes}_{\mathbb{F}_{q}((\zeta))} \breve{E}$, where $\mathcal{H}_{\mathrm{GL}_{r}, 2 n \rho \vee}^{\text {an }}:=$ $\left(\hat{Z}_{\mathrm{GL}_{r}, 2 n \rho^{\vee}}\right)^{\text {an }}$. On the connected components of $\breve{\mathcal{H}}_{G, \hat{Z}}^{\text {an }}$ where $[b]^{\#}=\gamma^{\#}$ in $\pi_{1}(G)_{\Gamma, \mathbb{Q}}$, we have by Definition 4.13

$$
\begin{aligned}
& \breve{\mathcal{H}}_{G, \hat{Z}, b}^{w a}=\breve{\mathcal{H}}_{G, \hat{Z}}^{\text {an }} \cap \breve{\mathcal{H}}_{\mathrm{GL}_{r}, 2 n \rho^{\vee}, \rho(b)}^{w a} \widehat{\otimes}_{\mathbb{F}((\zeta))} \breve{E} \quad \text { and } \\
& \breve{\mathcal{H}}_{G, \hat{Z}, b}^{a}=\breve{\mathcal{H}}_{G, \hat{Z}}^{\text {an }} \cap \breve{\mathcal{H}}_{\mathrm{GL}_{r}, 2 n \rho^{\vee}, \rho(b)}^{a} \widehat{\otimes}_{\mathbb{F}((\zeta))} \breve{E} .
\end{aligned}
$$

The intersections of the other components with $\breve{\mathcal{H}}_{G, \hat{Z}, b}^{w a}$ and $\breve{\mathcal{H}}_{G, \hat{Z}, b}^{a}$ are empty. Because every open subspace of the compact $\breve{E}$-analytic space $\breve{\mathcal{H}}_{G, \hat{Z}}^{\text {an }}$ is paracompact by [50, Lemma A.2.6], it suffices to show that $\breve{\mathcal{H}}_{\mathrm{GL}_{r}, 2 n \rho^{\vee}, \rho(b)}^{w a}$ and $\breve{\mathcal{H}}_{\mathrm{GL}_{r}, 2 n \rho^{\vee}, \rho(b)}^{a}$ are open in $\breve{\mathcal{H}}_{\mathrm{GL}_{r}, 2 n \rho^{\vee}}^{\text {an }} \widehat{\otimes}_{\mathbb{F}((\zeta))} \breve{E}$. An analogous statement was proved in [50, Theorems 3.2.2 and 3.2.4] for the quasi-projective Schubert cell

$$
\breve{\mathcal{C}}:=\mathrm{GL}_{r}(\cdot \llbracket z-\zeta \rrbracket) \cdot\left(2 n \rho^{\vee}\right)(z-\zeta) \cdot \mathrm{GL}_{r}(\cdot \llbracket z-\zeta \rrbracket) / \mathrm{GL}_{r}(\cdot \llbracket z-\zeta \rrbracket)
$$

from [50, Definition 3.1.6], which is open and dense in the Schubert variety $\breve{\mathcal{H}}_{\mathrm{GL}_{r}, 2 n \rho \vee}$. Let us explain how to modify that proof to obtain a proof of the assertion above. The Schubert cell is a homogeneous space $\breve{\mathcal{C}}=\widetilde{G} / S$; see [50, p. 1318]. The properties that were needed in the proofs of [50, Theorems 3.2.2 and 3.2.4] were the following two. The morphism $\widetilde{G}^{\text {an }} \rightarrow \breve{\mathcal{C}}^{\text {an }}$ is smooth, and therefore $\breve{\mathcal{C}}^{\text {an }}$ carries the quotient topology under the morphism $\widetilde{G}^{\text {an }} \rightarrow \breve{\mathcal{C}}^{\text {an }}$. Secondly, the universal Hodge-Pink structure on $\breve{\mathcal{C}}$ is given on $\widetilde{G}$ by a universal matrix $g$ in $\mathrm{GL}_{r}\left(\mathcal{O}_{\widetilde{G}} \llbracket z-\zeta \rrbracket /(z-\zeta)^{2 n(r-1)}\right)$.

For our purpose here we modify this as follows. Because $L G \rightarrow \mathcal{F} \ell_{G}$ has local sections for the étale topology, there is an étale covering $X$ of $\breve{\mathcal{H}}_{\mathrm{GL}_{r}, 2 n \rho^{\vee}}^{\text {an }}$ on which the universal Hodge-Pink $G$-structure is given by a universal element $h$ in $G\left(\mathcal{O}_{X}((z-\zeta))\right)$ that satisfies $\rho(h) \in M_{r}\left((z-\zeta)^{-n(r-1)} \mathcal{O}_{X} \llbracket z-\zeta \rrbracket\right)$. We replace $g$ by $(z-\zeta)^{n(r-1)} \rho(h) \bmod (z-\zeta)^{2 n(r-1)}$ and use that $\breve{\mathcal{H}}_{\mathrm{GL}_{r}, 2 n \rho \vee}^{\text {an }}$ carries the quotient topology under the morphism $X \rightarrow \breve{\mathcal{H}}_{\mathrm{GL}_{r}, 2 n \rho^{\vee}}^{\text {an }}$ by [8, Corollary 3.7.4]. With these modifications the proofs of [50, Theorems 3.2.2 and 3.2 .4 ] carry over to our situation.

The connectedness of $\breve{\mathcal{H}}_{G, \hat{Z}, b}^{w a}$ and $\breve{\mathcal{H}}_{G, \hat{Z}, b}^{a}$ can be proved by the same arguments as in [50, Theorem 3.2.5]. 
It remains to compute which connected components of $\operatorname{Gr}_{G, \breve{L}_{0}}^{\mathbf{B}_{\mathrm{dR}}}$ meet $\breve{\mathcal{H}}_{G, \hat{Z}, b}^{a}$ and $\breve{\mathcal{H}}_{G, \hat{Z}, b}^{w a}$. By Remark 4.15, for every point $\gamma \in \breve{\mathcal{H}}_{G, \hat{Z}, b}^{w a} \widehat{\otimes}_{\breve{E}} \breve{L}_{0}$ the Hodge point $\mu_{\gamma} \in X_{*}(T)$ lies in $N_{\text {an }}$ and $\nu_{b} \preceq \mu_{\gamma}$. In particular, $\gamma$ lies in the component with image $\mu_{\gamma}^{\#} \in \pi_{1}(G)$. Conversely, let $\mu \in N_{\text {an }}$ with $\nu_{b} \preceq \mu$. Then Remark 4.15 implies that there is a point $\gamma \in \operatorname{Gr}_{G, \breve{L}_{0}}^{\mathbf{B}_{\mathrm{dR}}}$ with Hodge point $\mu$ such that $(b, \gamma)$ is weakly admissible. By Remark 2.11(b), the point $\gamma$ lies in $\breve{\mathcal{H}}_{G, \hat{Z}, b}^{a}$ and $\breve{\mathcal{H}}_{G, \hat{Z}, b}^{w a}$ and, moreover, it lies in the connected component of $\operatorname{Gr}_{G, \breve{L}_{0}}^{\mathbf{B}_{\mathrm{dR}}}$ with image $\mu^{\#}$ in $\pi_{0}\left(\mathrm{Gr}_{G, \breve{L}_{0}}^{\mathbf{B}_{\mathrm{dR}}}\right)=\pi_{1}(G)$.

Remark 4.21. We keep the notation of Remark 3.7. The morphism $\varepsilon: G \rightarrow G^{\prime}$ of parahoric group schemes over $\mathbb{F}_{q} \llbracket z \rrbracket$ induces a morphism $\varepsilon$ : $\operatorname{Gr}_{G}^{\mathbf{B}_{\mathrm{dR}}} \rightarrow \mathrm{Gr}_{G^{\prime}}^{\mathbf{B}_{\mathrm{dR}}}$ of the affine Grassmannians from (1.2). It maps Hodge-Pink $G$-structures $\gamma \in \operatorname{Gr}_{G}^{\mathbf{B}_{\mathrm{dR}}}$ to HodgePink $G^{\prime}$-structures $\gamma^{\prime}:=\varepsilon(\gamma) \in \operatorname{Gr}_{G^{\prime}}^{\mathbf{B}_{\mathrm{dR}}}$ and the corresponding element $\gamma^{\#} \in \pi_{1}(G)_{\Gamma}$ to $\left(\gamma^{\prime}\right)^{\#}=\varepsilon\left(\gamma^{\#}\right) \in \pi_{1}\left(G^{\prime}\right)_{\Gamma}$.

The morphism $\varepsilon$ also induces a tensor functor $\varepsilon^{*}: \operatorname{Rep}_{\mathbb{F}_{q}((z))} G^{\prime} \rightarrow \operatorname{Rep}_{\mathbb{F}_{q}((z))} G$, given by $\left(V^{\prime}, \rho^{\prime}\right) \mapsto \varepsilon^{*}\left(V^{\prime}, \rho^{\prime}\right):=\left(V^{\prime}, \rho^{\prime} \circ \varepsilon\right)$. It satisfies $\underline{D}_{b, \gamma}\left(\varepsilon^{*}\left(V^{\prime}, \rho^{\prime}\right)\right)=\underline{D}_{b^{\prime}, \gamma^{\prime}}\left(V^{\prime}, \rho^{\prime}\right)$ for $b^{\prime}=\varepsilon(b)$. Therefore, $\underline{\mathcal{E}}_{b, \gamma}\left(\varepsilon^{*}\left(V^{\prime}, \rho^{\prime}\right)\right)=\underline{\mathcal{E}}_{b^{\prime}, \gamma^{\prime}}\left(V^{\prime}, \rho^{\prime}\right)$ and $\underline{\mathcal{F}}_{b, \gamma}\left(\varepsilon^{*}\left(V^{\prime}, \rho^{\prime}\right)\right)=\underline{\mathcal{F}}_{b^{\prime}, \gamma^{\prime}}\left(V^{\prime}, \rho^{\prime}\right)$. It follows that $(b, \gamma)$ is (weakly) admissible for $G$ if and only if $\left(b^{\prime}, \gamma^{\prime}\right)$ is for $G^{\prime}$. Moreover, if $(b, \gamma)$ is neutral, then $\left(b^{\prime}, \gamma^{\prime}\right)$ is also neutral. In other words, if $\varepsilon(\hat{Z}) \subset \hat{Z}^{\prime}$, then $\varepsilon$ induces morphisms

$$
\varepsilon: \breve{\mathcal{H}}_{G, \hat{Z}} \longrightarrow \breve{\mathcal{H}}_{G^{\prime}, \hat{Z}^{\prime}} \quad \text { and } \quad \varepsilon: \breve{\mathcal{H}}_{G, \hat{Z}, b}^{\bullet} \longrightarrow \breve{\mathcal{H}}_{G^{\prime}, \hat{Z}^{\prime}, b^{\prime}}^{\bullet}, \quad \gamma \longmapsto \varepsilon(\gamma)
$$

for $\bullet \in\{w a, a, n a\}$.

\section{Local systems of $\mathbb{F}_{q}((z))$-vector spaces}

Definition 5.1. For a ring $A$ we let $\mathrm{FMod}_{A}$ denote the category of finite locally free $A$-modules. If the ring $A$ is either $\mathbb{F}_{q} \llbracket z \rrbracket$ or $\mathbb{F}_{q}((z))$ and $\Pi$ is a topological group, we denote by $\operatorname{Rep}_{A}^{\text {cont }}(\Pi)$ the category of continuous representations in finite free $A$-modules and by

$$
\text { forget : } \operatorname{Rep}_{A}^{\text {cont }}\left(\pi_{1}^{\text {ét }}(X, \bar{x})\right) \longrightarrow \operatorname{FMod}_{A}
$$

the forgetful fibre functor. Moreover, we let

$$
\omega_{A}^{\circ}: \operatorname{Rep}_{A} G \longrightarrow \mathrm{FMod}_{A}
$$

be the forgetful fibre functor. We also write $\omega^{\circ}:=\omega_{\mathbb{F}_{q}((z))}^{\circ}$. Then $\operatorname{Aut}^{\otimes}\left(\omega^{\circ}\right)=G_{\mathbb{F}_{q}((z))}$ by $\left[33\right.$, Theorem 2.11] and $\mathrm{Aut}^{\otimes}\left(\omega_{\mathbb{F}_{q} \llbracket z \rrbracket}^{\circ}\right)=G$ by [96, Corollary 5.20].

Let $X$ be a strictly $L$-analytic space, where $L$ is a field extensions of $\mathbb{F}_{q}((\zeta))$ that is complete with respect to an absolute value $||:. L \rightarrow \mathbb{R}_{\geq 0}$ extending the $\zeta$-adic absolute value on $\mathbb{F}_{q}((\zeta))$. For any group or ring $A$ we denote by $\underline{A}$ the locally constant sheaf on the étale site $X_{\text {ét }}$ of $X$.

We recall de Jong's [31, §2] definition of the étale fundamental group of $X$. De Jong calls a morphism $f: Y \rightarrow X$ of $L$-analytic spaces an étale covering space of $X$ if for every analytic point $x$ of $X$ there exists an open neighbourhood $U \subset X$ such that $Y{ }_{X} U$ is a 
disjoint union of $L$-analytic spaces $V_{i}$ each mapping finite étale to $U$. The étale covering spaces of $X$ form a category $\underline{\operatorname{Cov}}_{X}^{\text {ét }}$. It contains the full subcategory $\underline{\operatorname{Cov}}_{X}^{\text {alg }}$ of finite étale covering spaces.

A geometric base point $\bar{x}$ of $X$ is a morphism $\bar{x}: \operatorname{BSpec}(\bar{L}) \rightarrow X$ where we denote by $\operatorname{BSpec}(\bar{L})$ the Berkovich spectrum of an algebraically closed complete extension $\bar{L}$ of $L$. For a geometric base point $\bar{x}$ of $X$, define the fibre functors at $\bar{x}$

$$
\begin{aligned}
& F_{\bar{x}}^{\text {ét }}:=F_{X, \bar{x}}^{\text {ét }}: \underline{\operatorname{Cov}_{X}^{\text {ét }}} \longrightarrow \underline{\text { Sets }}, \quad F_{\bar{x}}^{\text {ét }}(f: Y \rightarrow X):=\{\bar{y}: \operatorname{BSpec}(\bar{L}) \rightarrow Y \text { with } f \circ \bar{y}=\bar{x}\} \\
& F_{\bar{x}}^{\mathrm{alg}}:=F_{X, \bar{x}}^{\mathrm{alg}}: \underline{\operatorname{Cov}}_{X}^{\mathrm{alg}} \longrightarrow \underline{\operatorname{Sets}}, \quad F_{\bar{x}}^{\mathrm{alg}}:=\left.F_{\bar{x}}^{\text {ét }}\right|_{\underline{\operatorname{Cov}}_{X}^{\text {alg }}} .
\end{aligned}
$$

The étale fundamental group $\pi_{1}^{\text {ét }}(X, \bar{x})$ and the algebraic fundamental group $\pi_{1}^{\text {alg }}(X, \bar{x})$ of $X$ are the automorphism groups

$$
\pi_{1}^{\text {ét }}(X, \bar{x}):=\operatorname{Aut}\left(F_{\bar{x}}^{\text {ét }}\right) \quad \text { and } \quad \pi_{1}^{\text {alg }}(X, \bar{x}):=\operatorname{Aut}\left(F_{\bar{x}}^{\text {alg }}\right) .
$$

These fundamental groups classify (finite) étale covering spaces in the sense that $F_{\bar{x}}$ induces an equivalence

$$
F_{\bar{x}}^{\text {ét }}:\left\{\text { disjoint unions of objects of } \underline{\mathrm{Cov}}_{X}^{\text {ét }}\right\} \longrightarrow \pi_{1}^{\text {ét }}(X, \bar{x}) \text {-Sets. }
$$

Connected coverings correspond to $\pi_{1}^{\text {ét }}(X, \bar{x})$-orbits and similarly for $F_{\bar{x}}^{\text {alg }}$; see [31, Theorem 2.10]. Here $\pi_{1}^{\text {et }}(X, \bar{x})$-Sets (respectively $\pi_{1}^{\text {alg }}(X, \bar{x})$-Sets is the category of discrete (respectively finite) sets endowed with a continuous left action of $\pi_{1}^{\text {ét }}(X, \bar{x})$ (respectively $\left.\pi_{1}^{\text {alg }}(X, \bar{x})\right)$.

The natural continuous group homomorphism $\pi_{1}^{\text {et }}(X, \bar{x}) \rightarrow \pi_{1}^{\text {alg }}(X, \bar{x})$ has dense image. The étale fundamental group $\pi_{1}^{\text {ét }}(X, \bar{x})$ is Hausdorff and pro-discrete, $\pi_{1}^{\text {alg }}(X, \bar{x})$ is pro-finite and every continuous homomorphism from $\pi_{1}^{\text {et }}(X, \bar{x})$ to a pro-finite group factors through $\pi_{1}^{\mathrm{alg}}(X, \bar{x})$; see [31, Lemma 2.7 and Theorem 2.10]. In particular, $\operatorname{Rep}_{\mathbb{F}_{q} \llbracket z \rrbracket}^{\text {cont }}\left(\pi_{1}^{\text {ét }}(X, \bar{x})\right)=\operatorname{Rep}_{\mathbb{F}_{q} \llbracket z \rrbracket}^{\text {cont }}\left(\pi_{1}^{\text {alg }}(X, \bar{x})\right)$, but this is not true for representations on $\mathbb{F}_{q}((z))$-vector spaces.

For the following overview we follow [50, Definition A4.4].

Definition 5.2. A local system of $\mathbb{F}_{q} \llbracket z \rrbracket$-lattices on $X$ is a projective system $\mathcal{F}=\left(\mathcal{F}_{n}, i_{n}\right)$ of sheaves $\mathcal{F}_{n}$ of $\mathbb{F}_{q} \llbracket z \rrbracket /\left(z^{n}\right)$-modules on $X_{\text {ét }}$, such that

(a) $\mathcal{F}_{n}$ is étale locally a constant free $\mathbb{F}_{q} \llbracket z \rrbracket /\left(z^{n}\right)$-module of finite rank,

(b) $i_{n} \otimes \mathrm{id}: \mathcal{F}_{n} \otimes_{\mathbb{F}_{q} \llbracket z \rrbracket /\left(z^{n}\right)} \underline{\mathbb{F}_{q} \llbracket z \rrbracket /\left(z^{n-1}\right)} \stackrel{\sim}{\sim} \mathcal{F}_{n-1}$ is an isomorphism of sheaves of $\underline{\mathbb{F}_{q} \llbracket z \rrbracket /\left(z^{n-1}\right)} \overline{\text { modules. }}$

The category $\mathbb{F}_{q} \llbracket z \rrbracket$-Loc $\underline{-}_{X}$ of local systems of $\mathbb{F}_{q} \llbracket z \rrbracket$-lattices with the obvious morphisms is an additive $\mathbb{F}_{q} \llbracket z \rrbracket$-linear rigid tensor category. If $\bar{x}$ is a geometric point of $X$,

$$
\mathcal{F}_{\bar{x}}:=\lim _{\longleftarrow}\left(\mathcal{F}_{n, \bar{x}}, i_{n}\right)
$$

is the stalk $\mathcal{F}_{\bar{x}}$ of $\mathcal{F}$ at $\bar{x}$. It is a finite free $\mathbb{F}_{q} \llbracket z \rrbracket$-module. Starting from $\mathbb{F}_{q} \llbracket z \rrbracket$-lattices one defines local systems of $\mathbb{F}_{q}((z))$-vector spaces and their stalks as in $[31, \S 4]$ or $[50$, Definition A4.4]. 
Local systems of $\mathbb{F}_{q}((z))$-vector spaces form a category $\mathbb{F}_{q}((z))$-Loc $\underline{\text { Loc }}_{X}$. It is an abelian $\mathbb{F}_{q}((z))$-linear rigid tensor category. The theory of these local systems parallels the theory of local systems of $\mathbb{Q}_{\ell}$-vector spaces developed in [31]. In particular, there is the following description.

Proposition 5.3 (Compare [31, Corollary 4.2].). For any geometric point $\bar{x}$ of $X$ there is a natural $\mathbb{F}_{q} \llbracket z \rrbracket$-linear tensor functor

$$
\omega_{\bar{x}}: \mathbb{F}_{q} \llbracket z \rrbracket-\underline{\operatorname{Loc}}_{X} \longrightarrow \operatorname{Rep}_{\mathbb{F}_{q} \llbracket z \rrbracket}^{\text {cont }}\left(\pi_{1}^{\text {alg }}(X, \bar{x})\right)=\operatorname{Rep}_{\mathbb{F}_{q} \llbracket z \rrbracket}^{\text {cont }}\left(\pi_{1}^{\text {ét }}(X, \bar{x})\right)
$$

and a natural $\mathbb{F}_{q}((z))$-linear tensor functor

$$
\omega_{\bar{x}}: \mathbb{F}_{q}((z))-\underline{\operatorname{Loc}}_{X} \longrightarrow \operatorname{Rep}_{\mathbb{F}_{q}((z))}^{\text {cont }}\left(\pi_{1}^{\text {ét }}(X, \bar{x})\right)
$$

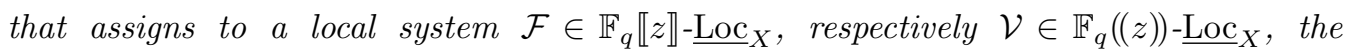
representation of $\pi_{1}^{\text {alg }}(X, \bar{x})$ on $\mathcal{F}_{\bar{x}}$, respectively of $\pi_{1}^{\text {et }}(X, \bar{x})$ on $\mathcal{V}_{\bar{x}}$. These tensor functors are equivalences if $X$ is connected.

Proof. De Jong [31, Corollary 4.2] proved this for $\mathbb{Q}_{\ell}$ and the statement for $\mathbb{F}_{q}((z))$ is proved verbatim. We indicate the (easier) argument for $\mathbb{F}_{q} \llbracket z \rrbracket$. Let $\mathcal{F}=\left(\mathcal{F}_{n}, i_{n}\right) \in$ $\mathbb{F}_{q} \llbracket z \rrbracket$-Loc ${ }_{X}$. Then the $\mathcal{F}_{n}$ are represented by finite étale covering spaces $Y_{n}$ of $X$. This yields an action of $\pi_{1}^{\mathrm{alg}}(X, \bar{x})$ on $F_{\bar{x}}^{\mathrm{alg}}\left(Y_{n}\right)=\mathcal{F}_{n, \bar{x}}$ and on $\mathcal{F}_{\bar{x}}:=\lim \left(\mathcal{F}_{n, \bar{x}}, i_{n}\right)$.

Let $A=\mathbb{F}_{q} \llbracket z \rrbracket$ or $A=\mathbb{F}_{q}((z))$ and recall the forgetful fibre functor $\omega_{A}^{\circ}: \operatorname{Rep}_{A} G \rightarrow \operatorname{FMod}_{A}$ from Definition 5.1. If $A=\mathbb{F}_{q}((z))$, we let in addition $\widetilde{\omega}: \operatorname{Rep}_{\mathbb{F}_{q}((z))} G \rightarrow \operatorname{FMod}_{\mathbb{F}_{q}((z))}$ be another fibre functor, and we let $\widetilde{G}:=\operatorname{Aut}^{\otimes}(\widetilde{\omega})$ be the group scheme over $\mathbb{F}_{q}((z))$ of tensor automorphisms of $\widetilde{\omega}$; see [33, Theorem 2.11]. Then $\operatorname{Isom}^{\otimes}\left(\omega^{\circ}, \widetilde{\omega}\right)$ is a left $G$-torsor and a right $\widetilde{G}$-torsor over $\mathbb{F}_{q}((z))$ and corresponds to a cohomology $\operatorname{class} c l\left(\omega^{\circ}, \widetilde{\omega}\right) \in \mathrm{H}^{1}\left(\mathbb{F}_{q}((z)), G\right)$ by [33, Theorem 3.2]. The group $\widetilde{G}$ is isomorphic to the inner form of $G$ defined by the image of $\operatorname{cl}\left(\omega^{\circ}, \widetilde{\omega}\right)$ in $\mathrm{H}^{1}\left(\mathbb{F}_{q}((z)), G^{\text {ad }}\right)$, where $G \rightarrow G^{\text {ad }}$ is the adjoint quotient. If $A=$ $\mathbb{F}_{q} \llbracket z \rrbracket$, we set $\widetilde{\omega}:=\omega_{\mathbb{F}_{q} \llbracket z \rrbracket}^{\circ}$ and $\widetilde{G}:=G$. This is no restriction because Lang's theorem $\left[72\right.$, Theorem 2], stating $\mathrm{H}^{1}\left(\mathbb{F}_{q} \llbracket z \rrbracket, G\right)=\mathrm{H}^{1}\left(\mathbb{F}_{q}, G\right)=(1)$, implies that all fibre functors $\operatorname{Rep}_{\mathbb{F}_{q} \llbracket z \rrbracket} G \rightarrow \operatorname{FMod}_{\mathbb{F}_{q} \llbracket z \rrbracket}$ are isomorphic to $\omega_{\mathbb{F}_{q} \llbracket z \rrbracket}^{\circ}$.

Let $X$ be connected, let $\bar{x}$ be a geometric base point of $X$ and recall the forgetful fibre functor forget: $\operatorname{Rep}_{A}^{\text {cont }}\left(\pi_{1}^{\text {ét }}(X, \bar{x})\right) \rightarrow \operatorname{FMod}_{A}$ from (5.1).

Corollary 5.4. In the situation above, consider the set $\mathcal{T}_{A}$ of isomorphism classes of pairs $(\underline{\mathcal{V}}, \beta)$ where $\underline{\mathcal{V}}: \operatorname{Rep}_{A} G \rightarrow A-\underline{\operatorname{Loc}}_{X}$ is a tensor functor and $\beta \in \operatorname{Isom}^{\otimes}\left(\widetilde{\omega}\right.$, forget $\left.^{\circ} \omega_{\bar{x}} \circ \underline{\mathcal{V}}\right)(A)$ is an isomorphism of tensor functors. There is a canonical bijection between $\mathcal{T}_{A}$ and the set of continuous group homomorphisms

$$
\pi_{1}^{\text {ét }}(X, \bar{x}) \longrightarrow \widetilde{G}(A) .
$$

Proof. Let $(\underline{\mathcal{V}}, \beta) \in \mathcal{T}_{A}$. By Proposition 5.3, any element of $\pi_{1}^{\text {ét }}(X, \bar{x})$ yields a tensor automorphism of the fibre functor forget $\circ \omega_{\bar{x}} \circ \underline{\mathcal{V}}$. By $\beta$ it is transported to a tensor automorphism of $\widetilde{\omega}$; that is, an element of $\widetilde{G}(A)$. This defines a group homomorphism $f:=f_{(\mathcal{V}, \beta)}: \pi_{1}^{\text {ét }}(X, \bar{x}) \rightarrow \widetilde{G}(A)$. Because for all $V \in \operatorname{Rep}_{A} G$ the induced homomorphism $\pi_{1}^{\text {ét }}(X, \bar{x}) \rightarrow \widetilde{G}(A) \rightarrow \mathrm{GL}(\widetilde{\omega}(V))(A)$ is continuous, $f$ is also continuous. 
Conversely, let $f: \pi_{1}^{\text {ét }}(X, \bar{x}) \rightarrow \widetilde{G}(A)$ be a continuous group homomorphism. Then we define a tensor functor $\operatorname{Rep}_{A} G \rightarrow \operatorname{Rep}_{A}^{\text {cont }}\left(\pi_{1}^{\text {ét }}(X, \bar{x})\right)$ by sending a representation $V$ in $\operatorname{Rep}_{A} G$ to the representation $\left(\widetilde{\omega}(V), \rho_{V}^{\prime}\right)$ given by

$$
\rho_{V}^{\prime}: \pi_{1}^{\text {ét }}(X, \bar{x}) \longrightarrow \operatorname{GL}(\widetilde{\omega}(V))(A), \quad g \mapsto \widetilde{\omega}(V)(f(g)) .
$$

Here $\widetilde{\omega}(V)(f(g))$ is the automorphism by which $f(g) \in \widetilde{G}(A)=\operatorname{Aut}^{\otimes}(\widetilde{\omega})(A)$ acts on the $A$-module $\widetilde{\omega}(V)$. Note that $\rho_{V}^{\prime}$ is continuous because $\widetilde{G}(A) \rightarrow \operatorname{GL}(\widetilde{\omega}(V))(A)$ is continuous. Let $\underline{\mathcal{V}}_{f}(V) \in A$ - $\underline{\mathrm{Loc}}_{X}$ be the local system on $X$ induced from $\rho_{V}^{\prime}$ via Proposition 5.3. This defines a tensor functor $\underline{\mathcal{V}}_{f}: \operatorname{Rep}_{A} G \rightarrow A$ - $\underline{\operatorname{Loc}}_{X}$ for which forgeto $\omega_{\bar{x}} \circ \underline{\mathcal{V}}$ is identified with $\widetilde{\omega}$. We let $\beta_{f} \in \operatorname{Isom}^{\otimes}\left(\widetilde{\omega}\right.$, forget $\left.\circ \omega_{\bar{x}} \circ \underline{\mathcal{V}}\right)(A)$ be this identification.

Clearly, the assignments $(\underline{\mathcal{V}}, \beta) \mapsto f_{(\underline{\mathcal{V}}, \beta)}$ and $f \mapsto\left(\underline{\mathcal{V}}_{f}, \beta_{f}\right)$ satisfy $f=f_{\left(\underline{\mathcal{V}}_{f}, \beta_{f}\right)}$. Conversely, if $(\underline{\mathcal{V}}, \beta) \in \mathcal{T}_{A}$ and $f=f_{(\underline{\mathcal{V}}, \beta)}$, then $\beta$ provides an isomorphism $\left(\underline{\mathcal{V}}_{f}, \beta_{f}\right) \stackrel{\sim}{\longrightarrow}(\underline{\mathcal{V}}, \beta)$, and so $(\underline{\mathcal{V}}, \beta)$ and $\left(\underline{\mathcal{V}}_{f}, \beta_{f}\right)$ coincide in $\mathcal{T}_{A}$.

Remark 5.5. (a) In the situation of Corollary 5.4, any tensor functor $\underline{\mathcal{V}}: \operatorname{Rep}_{\mathbb{F}_{q}((z))} G \rightarrow$ $\mathbb{F}_{q}((z))$-Loc $\underline{-}_{X}$ induces a tower of étale covering spaces of $X$ with Hecke action, as described in $[51$, Remark $2.7(\mathrm{a})]$. To recall the construction, assume that $\operatorname{Isom}^{\otimes}\left(\widetilde{\omega}\right.$, forget $^{\circ} \circ \omega_{\bar{x}} \circ$ $\underline{\mathcal{V}})\left(\mathbb{F}_{q}((z))\right)$ is nonempty. The whole construction does not depend on the choice of the base point $\bar{x}$ by [31, Theorem 2.9] and hence also applies if $X$ is not connected. Let $\widetilde{K} \subset \widetilde{G}\left(\mathbb{F}_{q}((z))\right)$ be a compact open subgroup. For a strictly $L$-analytic space $S$ over $X$ and a lift of $\bar{x}$ to a geometric base point $\bar{s}$ of $S$, a rational $\widetilde{K}$-level structure on $\underline{\mathcal{V}}$ over $S$ is a residue class modulo $\widetilde{K}$ of $\mathbb{F}_{q}((z))$-rational tensor isomorphisms

$$
\left(\beta: \widetilde{\omega} \stackrel{\sim}{\longrightarrow} \text { forget } \circ \omega_{\bar{x}} \circ \underline{\mathcal{V}}\right) \bmod \widetilde{K} \in \operatorname{Isom}^{\otimes}\left(\widetilde{\omega}, \text { forget } \circ \omega_{\bar{x}} \circ \underline{\mathcal{V}}\right)\left(\mathbb{F}_{q}((z))\right) / \widetilde{K}
$$

such that the class $\beta \widetilde{K}$ is invariant under the étale fundamental group $\pi_{1}^{\text {et }}(S, \bar{s})$. Here $\operatorname{Isom}^{\otimes}\left(\widetilde{\omega}\right.$, forget $\left.\circ \omega_{\bar{x}} \circ \underline{\mathcal{V}}\right)\left(\mathbb{F}_{q}((z))\right)$ carries an action of $\widetilde{K}$ through the action of $\widetilde{G}$ on $\widetilde{\omega}$ and an action of $\pi_{1}^{\text {ét }}(S, \bar{s})$ through its action on $\omega_{\bar{x}} \circ \underline{\mathcal{V}}$ via the map $\pi_{1}^{\text {ét }}(S, \bar{s}) \rightarrow \pi_{1}^{\text {ét }}(X, \bar{x})$ and Proposition 5.3.

Let $\breve{\mathcal{E}}_{\widetilde{K}}$ be the étale covering space of $X$ corresponding to the discrete $\pi_{1}^{\text {ét }}(X, \bar{x})$-set $\operatorname{Isom}^{\otimes}\left(\widetilde{\omega}\right.$, forget $\left.\circ \omega_{\bar{x}} \circ \underline{\mathcal{V}}\right)\left(\mathbb{F}_{q}((z))\right) / \widetilde{K}$. Then $\breve{\mathcal{E}}_{\widetilde{K}}$ represents the rational $\widetilde{K}$-level structures on $\underline{\mathcal{V}}$. Any choice of a fixed tensor isomorphism $\beta_{0} \in \operatorname{Isom}^{\otimes}\left(\widetilde{\omega}\right.$, forget $\left._{0} \circ \omega_{\bar{x}} \circ \underline{\mathcal{V}}\right)\left(\mathbb{F}_{q}((z))\right)$ associates with $\underline{\mathcal{V}}$ a representation $\pi_{1}^{\text {et }}(X, \bar{x}) \rightarrow \widetilde{G}\left(\mathbb{F}_{q}((z))\right)$ as in Corollary 5.4 and induces an identification of the $\pi_{1}^{\text {et }}(X, \bar{x})$-sets $\operatorname{Isom}^{\otimes}\left(\widetilde{\omega}\right.$, forget $\left.\circ \omega_{\bar{x}} \circ \underline{\mathcal{V}}\right)\left(\mathbb{F}_{q}((z))\right) / \widetilde{K}$ and $\widetilde{G}\left(\mathbb{F}_{q}((z))\right) / \widetilde{K}$. Moreover, if $\widetilde{K}^{\prime} \subset \widetilde{K} \subset \widetilde{G}\left(\mathbb{F}_{q}((z))\right)$ are compact open subgroups, there is a natural projection morphism $\breve{\pi}_{\widetilde{K}, \widetilde{K}^{\prime}}: \breve{\mathcal{E}}_{\widetilde{K}^{\prime}} \rightarrow \breve{\mathcal{E}}_{\widetilde{K}}, \beta \widetilde{K}^{\prime} \mapsto \beta \widetilde{K}$.

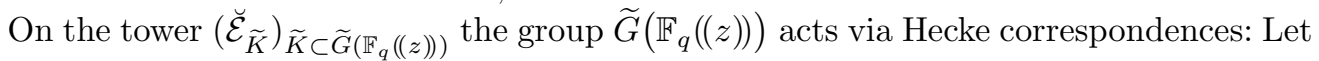
$g \in \widetilde{G}\left(\mathbb{F}_{q}((z))\right)$ and let $\widetilde{K} \subset \widetilde{G}\left(\mathbb{F}_{q}((z))\right)$ be a compact open subgroup. Then $g$ induces an isomorphism

$$
\iota(g)_{\widetilde{K}}: \breve{\mathcal{E}}_{\widetilde{K}} \stackrel{\sim}{\longrightarrow} \breve{\mathcal{E}}_{g^{-1} \widetilde{K}}, \quad \beta \widetilde{K} \longmapsto \beta \widetilde{K} g=\beta g\left(g^{-1} \widetilde{K} g\right) .
$$

(b) Assume, moreover, that a group $J$ acts on $X$ and let $\underline{\mathcal{V}}: \operatorname{Rep}_{\mathbb{F}_{q}((z))} G \rightarrow \mathbb{F}_{q}((z))-\underline{\operatorname{Loc}}_{X}$ be a tensor functor that carries a $J$-linearisation; that is, for every $j \in J$ an isomorphism 
$\varphi_{j}: j^{*} \underline{\mathcal{V}} \stackrel{\sim}{\longrightarrow}$ of tensor functors (where $j^{*} \underline{\mathcal{V}}$ is the pullback of $\underline{\mathcal{V}}$ under the morphism $j: X \rightarrow X)$, satisfying a cocycle condition. Then the tower of étale covering spaces $\breve{\mathcal{E}}_{\widetilde{K}}$ inherits an action of $J$ over $X$ as in [51, Remark 2.7(b)].

We now apply these considerations to the period spaces of Hodge-Pink $G$-structures.

Remark 5.6. The construction of the $\sigma$-bundle $\underline{\mathcal{F}}_{b, \gamma}(V)$ from Definition 4.9 works not only over a field extension $L$ of $\breve{E}$ but more generally over the entire $\breve{E}$-analytic space $\breve{\mathcal{H}}_{G, \hat{Z}}^{\text {an }}$ from Definition 4.16 . There it produces a $\sigma$-bundle $\underline{\mathcal{F}}_{b}(V)$ over $\breve{\mathcal{H}}_{G, \hat{Z}}^{\text {an }}$ whose fibre at each point $\gamma$ is $\underline{\mathcal{F}}_{b, \gamma}(V)$; see $[50, \S 2.4]$.

The restriction of $\underline{\mathcal{F}}_{b}(V)$ to $\breve{\mathcal{H}}_{G, \hat{z}, b}^{a}$ induces by Theorem [50, Theorem 3.4.1] a canonical local system $\underline{\mathcal{V}}_{b}(V)$ of $\mathbb{F}_{q}((z))$-vector spaces on $\breve{\mathcal{H}}_{G, \hat{Z}, b}^{a}$. It can be described as follows. On every connected component $Y$ of $\breve{\mathcal{H}}_{G, \hat{Z}, b}^{a}$ we choose a geometric base point $\bar{\gamma}: \operatorname{BSpec}(\bar{L}) \rightarrow$ $Y$. We consider the pullback $\bar{\gamma}^{*} \underline{\mathcal{F}}_{b}(V)$ and its $\tau$-invariants

$$
\bar{\gamma}^{*} \underline{\mathcal{F}}_{b}(V)^{\tau}:=\left\{f \in \bar{\gamma}^{*} \underline{\mathcal{F}}_{b}(V):\left(\bar{\gamma}^{*} \tau_{\mathcal{F}_{b}}\right)\left(\sigma^{*} f\right)=f\right\} .
$$

Because $\bar{\gamma}^{*} \underline{\mathcal{F}}_{b}(V) \cong \underline{\mathcal{F}}_{0,1} \oplus \operatorname{dim} V$, the $\tau$-invariants $\bar{\gamma}^{*} \underline{\mathcal{F}}_{b}(V)^{\tau}$ form an $\mathbb{F}_{q}((z))$-vector space of dimension equal to $\operatorname{dim} V$, which is equipped with a continuous action of the étale fundamental group $\pi_{1}^{\text {ét }}(Y, \bar{\gamma})$. This defines the local system $\underline{\mathcal{V}}_{b}(V)$ of $\mathbb{F}_{q}((z))$-vector spaces on $Y$ under the correspondence of Proposition 5.3. It satisfies $\underline{\mathcal{V}}_{b}(V)_{\bar{\gamma}}=\bar{\gamma}^{*} \underline{\mathcal{F}}_{b}(V)^{\tau}$ and $\underline{\mathcal{V}}_{b}(V)_{\bar{\gamma}} \otimes_{\mathbb{F}_{q}((z))} \bar{L}\left\langle\frac{z}{\bar{\zeta}^{s}}, z^{-1}\right\}=\bar{\gamma}^{*} \underline{\mathcal{F}}_{b}(V)^{\tau} \otimes_{\mathbb{F}_{q}((z))} \bar{L}\left\langle\frac{z}{\zeta^{s}}, z^{-1}\right\}=\bar{\gamma}^{*} \underline{\mathcal{F}}_{b}(V)=\underline{\mathcal{F}}_{b, \bar{\gamma}}(V)$.

If $\gamma \in \breve{\mathcal{H}}_{G, \hat{Z}, b}^{a}$ is the image of the geometric point $\bar{\gamma}$ and $L$ is the residue field of $\gamma$, then the fibre $\underline{\mathcal{V}}_{b}(V)_{\gamma}$ of $\underline{\mathcal{V}}_{b}(V)$ at $\gamma$ is a continuous representation of $\operatorname{Gal}\left(L^{\mathrm{sep}} / L\right)$ on the finite-dimensional $\mathbb{F}_{q}((z))$-vector space $\bar{\gamma}^{*} \underline{\mathcal{F}}_{b}(V)$. By [54, Remark 3.6.17] and with the notation from (6.1), this Galois-representation can be described as

$$
\left.\underline{\mathcal{V}}_{b}(V)_{\gamma}=\left(\underline{D} \otimes_{k((z))} \mathcal{O}_{\bar{L}} \llbracket z, z^{-1}\right\}\left[t^{-1}\right]\right)^{\tau} \cap \mathfrak{q}_{D} \otimes_{L \llbracket z-\zeta \rrbracket} \bar{L} \llbracket z-\zeta \rrbracket
$$

for $\underline{D}_{b, \gamma}(V)=\underline{D}=\left(D, \tau_{D}, \mathfrak{q}_{D}\right)$. Here we use the notation from (6.1) and in addition we let $t_{i} \in \mathcal{O}_{\mathbb{F}_{q}((\zeta))^{\text {sep }}}$ be solutions of the equations $t_{0}^{q-1}=-\zeta$ and $t_{i}^{q}+\zeta t_{i}=t_{i-1}$ and set $t_{+}:=\sum_{i=0}^{\infty} t_{i} z^{i} \in \mathcal{O}_{\mathbb{F}_{q}((\zeta)) \text { sep }} \llbracket z \rrbracket$ and $\left.t:=t_{+} t_{-} \in \mathcal{O}_{\bar{L}} \llbracket z, z^{-1}\right\}$, where $t_{-}$was defined in (4.3). In particular, if $L$ is discretely valued, then $\underline{\mathcal{V}}_{b}(V)_{\gamma}$ is the (equal characteristic) crystalline Galois representation associated with the (weakly) admissible $z$-isocrystal with HodgePink structure $\underline{D}_{b, \gamma}(V)$ in the sense of [54, Definition 3.4.21 and Remark 3.6.17].

Theorem 5.7. Let $b \in L G(\mathbb{F})$ and let $\hat{Z}=\left[\left(R, \hat{Z}_{R}\right)\right]$ be a bound as in Definition 2.2 with reflex ring $R_{\hat{Z}}=\kappa \llbracket \xi \rrbracket$. Then the assignments $V \mapsto \underline{\mathcal{F}}_{b}(V) \mapsto \bar{\gamma}^{*} \underline{\mathcal{F}}_{b}(V)^{\tau} \mapsto \underline{\mathcal{V}}_{b}(V)$ define an $\mathbb{F}_{q}((z))$-linear tensor functor $\underline{\mathcal{V}}_{b}$ from $\operatorname{Rep}_{\mathbb{F}_{q}((z))} G$ to the category $\mathbb{F}_{q}((z))-\underline{\operatorname{Loc}}_{\breve{\mathcal{H}}_{G, \hat{z}, b}^{a}}$ of local systems of $\mathbb{F}_{q}((z))$-vector spaces on $\breve{\mathcal{H}}_{G, \hat{z}, b}^{a}$. There is a canonical $J_{b}\left(\mathbb{F}_{q}((z))\right)$-linearisation on $\underline{\mathcal{V}}_{b}$.

Proof. First of all, the functors $V \mapsto \underline{D}_{b, \gamma}(V)$ from Remark 4.4 and $\underline{D} \mapsto(\underline{\mathcal{E}}(\underline{D}), \underline{\mathcal{F}}(\underline{D}))$ from Definition 4.9 are $\mathbb{F}_{q}((z))$-linear tensor functors, and this works equally for the entire families over $\breve{\mathcal{H}}_{G, \hat{Z}, b}^{a}$. In particular, $V \mapsto \underline{\mathcal{F}}_{b}(V)$ is a tensor functor from $\operatorname{Rep}_{\mathbb{F}_{q}((z))} G$ to the 
category of $\sigma$-bundles over $\breve{\mathcal{H}}_{G, \hat{z}, b}^{a}$. Next, taking $\tau$-invariants is obviously compatible with morphisms. It is in general not compatible with tensor products, but because $\bar{\gamma}^{*} \underline{\mathcal{F}}_{b}(V) \cong$ $\underline{\mathcal{F}}_{0,1} \oplus \operatorname{dim} V$, we have $\bar{\gamma}^{*} \underline{\mathcal{F}}_{b}(V) \cong\left(\bar{\gamma}^{*} \underline{\mathcal{F}}_{b}(V)^{\tau}\right) \otimes_{\mathbb{F}_{q}((z))} \bar{L}\left\langle\frac{z}{\zeta^{s}}, z^{-1}\right\}$. Therefore,

$$
\begin{aligned}
\bar{\gamma}^{*} \underline{\mathcal{F}}_{b}\left(V \otimes V^{\prime}\right) & \cong \bar{\gamma}^{*} \underline{\mathcal{F}}_{b}(V) \otimes_{\bar{L}\left\langle\frac{z}{\zeta^{s}}, z^{-1}\right\}} \bar{\gamma}^{*} \underline{\mathcal{F}}_{b}\left(V^{\prime}\right) \\
& \cong\left(\bar{\gamma}^{*} \underline{\mathcal{F}}_{b}(V)^{\tau} \otimes_{\mathbb{F}_{q}((z))} \bar{\gamma}^{*} \underline{\mathcal{F}}_{b}(V)^{\tau}\right) \otimes_{\mathbb{F}_{q}((z))} \bar{L}\left\langle\frac{z}{\zeta^{s}}, z^{-1}\right\},
\end{aligned}
$$

and hence $\bar{\gamma}^{*} \underline{\mathcal{F}}_{b}\left(V \otimes V^{\prime}\right)^{\tau} \cong \bar{\gamma}^{*} \underline{\mathcal{F}}_{b}(V)^{\tau} \otimes_{\mathbb{F}_{q}((z))} \bar{\gamma}^{*} \underline{\mathcal{F}}_{b}(V)^{\tau}$. We now use Proposition 5.3 to conclude that $\underline{\mathcal{V}}_{b}$ is an $\mathbb{F}_{q}((z))$-linear tensor functor.

If $j \in J_{b}\left(\mathbb{F}_{q}((z))\right)$ the isomorphism

$$
\left(j^{*} \underline{\mathcal{F}}_{b}(V)\right)_{\gamma}=\left(\underline{\mathcal{F}}_{b}(V)\right)_{j(\gamma)}=\underline{\mathcal{F}}_{b, j(\gamma)}(V) \cong \underline{\mathcal{F}}_{b, \gamma}(V)=\left(\underline{\mathcal{F}}_{b}(V)\right)_{\gamma}
$$

from Remark 4.17 yields $J_{b}\left(\mathbb{F}_{q}((z))\right)$-linearisations $\varphi_{j}: j^{*} \underline{\mathcal{F}}_{b}(V) \stackrel{\sim}{\longrightarrow} \underline{\mathcal{F}}_{b}(V)$ and $\varphi_{j}: j^{*} \underline{\mathcal{V}}_{b} \stackrel{\sim}{\longrightarrow} \underline{\mathcal{V}}_{b}$.

Let us end this section by stating the significance of Theorem 5.7 in terms of the étale fundamental group and in terms of étale covering spaces. Let $\bar{\gamma}$ be a geometric base point of $\breve{\mathcal{H}}_{G, \hat{Z}, b}^{a}$.

Remark 5.8. Let $\omega^{\circ}$ and $\omega_{b, \bar{\gamma}}:=$ forget $\circ \omega_{\bar{\gamma}} \circ \underline{\mathcal{V}}_{b}$ be the fibre functors from $\operatorname{Rep}_{\mathbb{F}_{q}((z))} G$ to $\operatorname{FMod}_{\mathbb{F}_{q}((z))}$ with $\omega^{\circ}(V):=V$ and $\omega_{b, \bar{\gamma}}(V):=\underline{\mathcal{V}}_{b}(V)_{\bar{\gamma}}=\bar{\gamma}^{*} \underline{\mathcal{F}}_{b}(V)^{\tau}$. By [31, Theorem 2.9], the fibre functors $\omega_{b, \bar{\gamma}}$ and $\omega_{b, \bar{\gamma}^{\prime}}$ are isomorphic for any two geometric base points $\bar{\gamma}$ and $\bar{\gamma}^{\prime}$ that lie in the same connected component of $\breve{\mathcal{H}}_{G, \hat{z}, b}^{a}$. Let $\widetilde{G}:=\operatorname{Aut}^{\otimes}\left(\omega_{b, \bar{\gamma}}\right)$. Then $\operatorname{Isom}^{\otimes}\left(\omega^{\circ}, \omega_{b, \bar{\gamma}}\right)$ is a left $G$-torsor and a right $\widetilde{G}$-torsor over $\mathbb{F}_{q}((z))$ and corresponds to a cohomology class $c l(b, \bar{\gamma}) \in \mathrm{H}^{1}\left(\mathbb{F}_{q}((z)), G\right)$ by [33, Theorem 3.2]. $\widetilde{G}$ is the inner form of $G$ defined by the image $\operatorname{cl}(b, \bar{\gamma}) \in \mathrm{H}^{1}\left(\mathbb{F}_{q}((z)), G^{\text {ad }}\right)$.

In the analogous situation over $\mathbb{Q}_{p}$, the torsor between the crystalline and the étale fibre functor is given by the cohomology class

$$
c l(b, \bar{\gamma})=[b]^{\#}-\bar{\gamma}^{\#} \in \mathrm{H}^{1}\left(\mathbb{Q}_{p}, G\right) .
$$

This was proved by Rapoport and Zink [80, 1.20] if $G_{\text {der }}$ is simply connected and in general by Wintenberger [97, Corollary to Proposition 4.5.3]; see also [28, Proposition on page 4].

The analogue of Wintenberger's theorem in our situation (which has not been proved yet $)$ is the statement that the torsor $\operatorname{Isom}^{\otimes}\left(\omega^{\circ}, \omega_{b, \bar{\gamma}}\right)$ is given by the cohomology class

$$
c l(b, \bar{\gamma})=[b]^{\#}-\bar{\gamma}^{\#} \in \mathrm{H}^{1}\left(\mathbb{F}_{q}((z)), G\right) .
$$

Note that also in the function field case considered here, the weak admissibility of the pair $(b, \bar{\gamma})$ implies that the difference $[b]^{\#}-\bar{\gamma}^{\#}$ lies in $\left(\pi_{1}(G)_{\Gamma}\right)_{\text {tors }}$, which is identified with $\mathrm{H}^{1}\left(\mathbb{F}_{q}((z)), G\right)$; see [78, Theorem 1.15] (and use [15, 8.6] instead of Steinberg's theorem). In particular, if $\bar{\gamma} \in \breve{\mathcal{H}}_{G, \hat{Z}, b}^{n a}$ and the analogue (5.6) of Wintenberger's theorem is established, then there is an $\mathbb{F}_{q}((z))$-rational tensor isomorphism $\beta: \omega^{\circ} \stackrel{\sim}{\longrightarrow} \omega_{b, \bar{\gamma}}$ and $\widetilde{G}:=$ Aut $^{\otimes}$ $\left(\omega_{b, \bar{\gamma}}\right) \cong G$. 
Remark 5.9. By Corollary 5.4, the restriction of the tensor functor $\underline{\mathcal{V}}_{b}$ from Theorem 5.7 to the connected component $Y$ of $\breve{\mathcal{H}}_{G, \hat{z}, b}^{a}$ containing the geometric base point $\bar{\gamma}$ corresponds to a continuous group homomorphism

$$
\pi_{1}^{\text {ét }}(Y, \bar{\gamma}) \longrightarrow \widetilde{G}\left(\mathbb{F}_{q}((z))\right)
$$

(under the choice of $\beta:=\operatorname{id}_{\widetilde{\omega}}$ for $\widetilde{\omega}:=\widetilde{\omega}_{b, \bar{\gamma}}$ ).

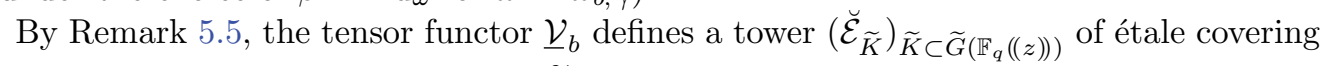
spaces of $\breve{\mathcal{H}}_{G, \hat{Z}, b}^{a}$. However, the group $\widetilde{G}$ might vary on the different connected components of $\breve{\mathcal{H}}_{G, \hat{Z}, b}^{a}$. It is therefore more useful to fix a base point $\bar{\gamma}$ and to define $\breve{\mathcal{H}}_{G, \hat{z}, b}^{a, \bar{\gamma}}$ as the union of those connected components consisting of elements $\bar{\gamma}^{\prime}$ with $\widetilde{\omega}_{b, \bar{\gamma}^{\prime}} \cong \widetilde{\omega}_{b, \bar{\gamma}}$. Then we obtain a tower $\left(\breve{\mathcal{E}}_{\widetilde{K}}^{\bar{\gamma}}\right)_{\widetilde{K} \subset \widetilde{G}\left(\mathbb{F}_{q}((z))\right)}$ of étale covering spaces of $\breve{\mathcal{H}}_{G, \hat{Z}, b}^{a, \bar{\gamma}}$ with commuting actions of $\widetilde{G}\left(\mathbb{F}_{q}((z))\right)$ by Hecke correspondences and of the quasi-isogeny group $J_{b}\left(\mathbb{F}_{q}((z))\right)$ of $\underline{\mathbb{G}}_{0}=\left(\left(L^{+} G\right)_{\mathbb{F}}, b \sigma^{*}\right)$ from $(3.1)$.

Note that for $A=\mathbb{F}_{q}((z))$ it can happen that $\widetilde{\omega} ¥ \omega^{\circ}$ but $\widetilde{G} \cong G$, namely, if $c l\left(\omega^{\circ}, \widetilde{\omega}\right) \in$ $\mathrm{H}^{1}\left(\mathbb{F}_{q}((z)), G\right)$ is nontrivial and lies in the image of $\mathrm{H}^{1}\left(\mathbb{F}_{q}((z)), Z\right)$, where $Z \subset G_{\mathbb{F}_{q}((z))}$ is the center. In this case, Corollary 5.4 could be applied to a continuous group homomorphism $\pi_{1}^{\text {ét }}(X, \bar{x}) \rightarrow G\left(\mathbb{F}_{q}((z))\right)$ using both $\omega^{\circ}$ and $\widetilde{\omega}$. One obtains two tensor functors $\underline{\mathcal{V}}, \underline{\widetilde{\mathcal{V}}}: \operatorname{Rep}_{A} G \rightarrow A$ - $\underline{\operatorname{Loc}}_{X}$ and tensor isomorphisms

$$
\beta \in \operatorname{Isom}^{\otimes}\left(\omega^{\circ}, \text { forget } \circ \omega_{\bar{x}} \circ \underline{\mathcal{V}}\right)\left(\mathbb{F}_{q}((z))\right)
$$

and

$$
\tilde{\beta} \in \operatorname{Isom}^{\otimes}\left(\widetilde{\omega}, \text { forget } \circ \omega_{\bar{x}} \circ \underline{\widetilde{\mathcal{V}}}\right)\left(\mathbb{F}_{q}((z))\right) .
$$

Because $\operatorname{cl}\left(\omega^{\circ}, \widetilde{\omega}\right)$ is trivialised by a finite unramified extension $\mathbb{F}_{q^{n}}((z))$ of $\mathbb{F}_{q}((z))$ by $[15$, 8.6], the pairs $(\underline{\mathcal{V}}, \beta)$ and $(\underline{\mathcal{V}}, \tilde{\beta})$ become isomorphic after tensoring with $\mathbb{F}_{q^{n}}((z))$.

\section{The period morphisms}

In this section we fix a local $G$-shtuka $\underline{\mathbb{G}}_{0}=\left(\left(L^{+} G\right)_{\mathbb{F}}, b \sigma^{*}\right)$ over $\mathbb{F}$ and a bound $\hat{Z}$ with reflex ring $R_{\hat{Z}}=\kappa \llbracket \xi \rrbracket$. We set $E_{\hat{Z}}=\kappa((\xi))$ and $\breve{E}:=\mathbb{F}((\xi))$. For any point $(\underline{\mathcal{G}}, \bar{\delta}) \in \breve{\mathcal{M}}_{\underline{\mathbb{G}}_{0}}^{\hat{Z}^{-1}}(S)$ with values in $S \in \mathcal{N} i l p_{\breve{R}_{\hat{Z}}}$, the quasi-isogeny $\bar{\delta}: \underline{\mathcal{G}}_{\bar{S}} \rightarrow \underline{\mathbb{G}}_{0, \bar{S}}$ lifts to a quasi-isogeny $\delta: \underline{\mathcal{G}} \rightarrow \underline{\mathbb{G}}_{0, S}$ by rigidity [4, Proposition 2.11]. To construct period morphisms for local $G$-shtukas we need to lift the universal $\bar{\delta}$, which is defined over the zero locus $\mathrm{V}(\zeta)$ of $\zeta$ in $\breve{\mathcal{M}}_{\mathbb{G}_{0}}^{\hat{Z}^{-1}}$, to the entire formal scheme $\breve{\mathcal{M}}_{\mathbb{G}_{0}}^{\hat{Z}^{-1}}$. This lift will no longer be a quasiisogeny, because it acquires larger and larger powers of $z$ in the denominators by lifting successively modulo $\zeta^{q^{n}}$. To describe what the limit of this lifting procedure is, we need the following generalisation of [50, Lemma 2.3.1] and [41, Lemmas 2.8 and 6.4]. For an $\mathbb{F} \llbracket \zeta \rrbracket$-algebra $B$ that is complete and separated with respect to a bounded norm $||:. B \rightarrow\{x \in \mathbb{R}: 0 \leq x \leq 1\}$ with $0<|\zeta|<1$, we define the $\mathbb{F}((z))$-algebra

$$
\left.B \llbracket z, z^{-1}\right\}:=\left\{\sum_{i \in \mathbb{Z}} b_{i} z^{i}: b_{i} \in B,\left|b_{i}\right||\zeta|^{r i} \rightarrow 0(i \rightarrow-\infty) \text { for all } r>0\right\} \text {. }
$$


Note that the convergence $\left|b_{i}\right||\zeta|^{r i} \rightarrow 0$ for $i \rightarrow-\infty$ implies that $\left|b_{i}\right|<1$ for $i \gg 0$. The element $t_{-}:=\prod_{i \in \mathbb{N}_{0}}\left(1-\frac{\zeta^{q^{i}}}{z}\right)$ lies in $\left.B \llbracket z, z^{-1}\right\}$ and satisfies $z t_{-}=(z-\zeta) \sigma^{*}\left(t_{-}\right)$; see (4.3). Note that $\left.B \llbracket z, z^{-1}\right\}$ contains $B \llbracket z \rrbracket$ and $z^{-1}$ and that $\left.B \llbracket z, z^{-1}\right\} /(\zeta)=\bar{B} \llbracket z \rrbracket\left[\frac{1}{z}\right]=: \bar{B}((z))$, for $\bar{B}:=B / \zeta B$. Moreover, note that $\sigma^{*}\left(t_{-}\right) \in B\left[\frac{1}{\zeta}\right] \llbracket z-\zeta \rrbracket^{\times}$, and $\left.B \llbracket z, z^{-1}\right\} \subset B\left[\frac{1}{\zeta}\right] \llbracket z-\zeta \rrbracket$ by $\sum_{i} b_{i} z^{i}=\sum_{i} b_{i}(\zeta+(z-\zeta))^{i}=\sum_{n \geq 0} \sum_{i} b_{i}\left(\begin{array}{c}i \\ n\end{array}\right) \zeta^{i-n}(z-\zeta)^{n}$. Therefore,

$$
\left.B \llbracket z, z^{-1}\right\}\left[\frac{1}{\sigma^{*}\left(t_{-}\right)}\right] \subset B\left[\frac{1}{\zeta}\right] \llbracket z-\zeta \rrbracket .
$$

Lemma 6.1. Let $B$ be an $\mathbb{F} \llbracket \zeta \rrbracket$-algebra as above. Let $b \in L G(\mathbb{F})=G(\mathbb{F}((z))), A \in$ $G\left(B \llbracket z \rrbracket\left[\frac{1}{z-\zeta}\right]\right)$ and $\bar{\Delta} \in L G(\bar{B})=G(\bar{B}((z)))$ such that $\bar{\Delta} \cdot(A \bmod \zeta)=b \cdot \sigma^{*}(\bar{\Delta})$ in $G(\bar{B}((z)))$. Then there is a unique $\left.\Delta \in G\left(B \llbracket z, z^{-1}\right\}\left[\frac{1}{t_{-}}\right]\right)$with $\Delta \bmod \zeta=\bar{\Delta}$ and $\Delta \cdot A=$ $b \cdot \sigma^{*}(\Delta)$ in $\left.G\left(B \llbracket z, z^{-1}\right\}\left[\frac{1}{t_{-}}\right]\right)$.

Proof. We choose a faithful representation $\rho: G \hookrightarrow \mathrm{GL}_{r, \mathbb{F}_{q} \llbracket z \rrbracket}$ over $\mathbb{F}_{q} \llbracket z \rrbracket$. There is a positive integer $N$ such that $\rho(b), \rho\left(b^{-1}\right) \in M_{r}\left(z^{-N} \mathbb{F} \llbracket z \rrbracket\right)$, as well as $\rho(A), \rho\left(A^{-1}\right) \in$ $M_{r}\left((z-\zeta)^{-N} B \llbracket z \rrbracket\right)$, and $\rho(\bar{\Delta}), \rho\left(\bar{\Delta}^{-1}\right) \in M_{r}\left(z^{-2 N} \bar{B} \llbracket z \rrbracket\right)$. We choose $C_{0}, D_{0} \in$ $M_{r}\left(z^{-2 N} B \llbracket z \rrbracket\right)$ with $C_{0} \equiv \rho(\bar{\Delta})(\bmod \zeta)$ and $D_{0} \equiv \rho\left(\bar{\Delta}^{-1}\right)(\bmod \zeta)$. For $m>0$ we inductively define

$$
\begin{aligned}
C_{m} & :=\left(z^{-N} \rho(b)\right) \sigma^{*} C_{m-1}\left((z-\zeta)^{N} \rho\left(A^{-1}\right)\right) \\
D_{m} & :=\left((z-\zeta)^{N} \rho(A)\right) \sigma^{*} D_{m-1}\left(z^{-N} \rho\left(b^{-1}\right)\right)
\end{aligned}
$$

in $M_{r}\left(z^{-2 N(m+1)} B \llbracket z \rrbracket\right)$. The assumption on $\bar{\Delta}$ implies

$$
C_{1}-C_{0} \equiv \rho\left(b \sigma^{*}(\bar{\Delta}) A^{-1}\right)-\rho(\bar{\Delta}) \equiv 0(\bmod \zeta)
$$

By induction on $m$, we obtain that

$$
\begin{aligned}
C_{m+1}-C_{m} & =\left(z^{-N} \rho(b)\right) \sigma^{*}\left(C_{m}-C_{m-1}\right)\left((z-\zeta)^{N} \rho\left(A^{-1}\right)\right) \\
& \equiv 0\left(\bmod \sigma^{*}\left(\sigma^{m-1}\right)^{*}(\zeta)\right) \\
& \equiv 0\left(\bmod \left(\sigma^{m}\right)^{*}(\zeta)\right)
\end{aligned}
$$

Therefore, the sequence $\left(C_{m}\right)_{m}$ converges to a matrix $\left.C \in M_{r}\left(B \llbracket z, z^{-1}\right\}\right)$ and the sequence $\left(\prod_{i=0}^{m}\left(1-\frac{\zeta^{q^{i}}}{z}\right)^{-N} \cdot C_{m}\right)_{m}$ converges to the matrix $\left.t_{-}^{-N} \cdot C \in M_{r}\left(B \llbracket z, z^{-1}\right\}\left[\frac{1}{t_{-}}\right]\right)$ with $\left(t_{-}^{-N} C\right) \equiv C \equiv \rho(\bar{\Delta})(\bmod \zeta)$. The equation

$$
\prod_{i=0}^{m}\left(1-\frac{\zeta^{q^{i}}}{z}\right)^{-N} \cdot C_{m} \cdot \rho(A)=\rho(b) \cdot \sigma^{*}\left(\prod_{i=0}^{m-1}\left(1-\frac{\zeta^{q^{i}}}{z}\right)^{-N} \cdot C_{m-1}\right)
$$

implies $\left(t_{-}^{-N} C\right) \rho(A)=\rho(b) \sigma^{*}\left(t_{-}^{-N} C\right)$ in $\left.M_{r}\left(B \llbracket z, z^{-1}\right\}\left[\frac{1}{t_{-}}\right]\right)$. In the same way, one sees that $\left(D_{m}\right)_{m}$ converges to a matrix $\left.D \in M_{r}\left(B \llbracket z, z^{-1}\right\}\right)$ with $\left(t_{-}^{-N} D\right) \rho(b)=\rho(A) \sigma^{*}\left(t_{-}^{-N} D\right)$ in $\left.M_{r}\left(B \llbracket z, z^{-1}\right\}\left[\frac{1}{t_{-}}\right]\right)$and $\left(t_{-}^{-N} D\right) \bmod \zeta=\rho\left(\bar{\Delta}^{-1}\right)$. We obtain the congruences 


$$
\begin{aligned}
& \operatorname{Id}_{r}-t_{-}^{-2 N} C D \equiv 0(\bmod \zeta) \quad \text { and by induction } \\
& \operatorname{Id}_{r}-t_{-}^{-2 N} C D=\rho(b) \cdot \sigma^{*}\left(\operatorname{Id}_{r}-t_{-}^{-2 N} C D\right) \rho(b)^{-1} \equiv 0\left(\bmod \zeta^{q^{m}}\right) \text { for all } m .
\end{aligned}
$$

By looking at power series expansions in $z$ of the matrix coefficients, these congruences imply by the separatedness of the norm $|$.$| that \left(t_{-}^{-N} C\right)\left(t_{-}^{-N} D\right)=\operatorname{Id}_{r}$; that is, $t_{-}^{-N} C \in$ $\left.\mathrm{GL}_{r}\left(B \llbracket z, z^{-1}\right\}\left[\frac{1}{t_{-}}\right]\right)$. Because $\left(\sigma^{m *} C\right) \bmod \zeta^{q^{m}}=\sigma^{m *}(C \bmod \zeta)=\rho\left(\sigma^{m *} \bar{\Delta}\right)$, it follows that

$$
\begin{aligned}
\left(t_{-}^{-N} C\right) \bmod \zeta^{q^{m}} & =\left(\prod_{i=0}^{m-1}\left(1-\frac{\zeta^{q^{i}}}{z}\right)^{-N} \cdot C_{m-1}\right) \bmod \zeta^{q^{m}} \\
& =\rho\left(b \cdot \ldots \cdot \sigma^{(m-1) *}(b) \cdot \sigma^{m *}(\bar{\Delta}) \cdot \sigma^{(m-1) *}\left(A^{-1}\right) \cdot \ldots \cdot A^{-1}\right) \bmod \zeta^{q^{m}}
\end{aligned}
$$

satisfies the equations that cut out the closed subgroup scheme $\rho(G)$ of $\mathrm{GL}_{r}$. Because $B$ is separated with respect to the norm $|$.$| , we must have t_{-}^{-N} C=\rho(\Delta)$ for a matrix $\left.\Delta \in G\left(B \llbracket z, z^{-1}\right\}\left[\frac{1}{t_{-}}\right]\right)$.

Finally, to prove the uniqueness of $\Delta$, we assume that $\Delta^{\prime}$ also satisfies the assertions of the lemma. Then $U:=\rho(\Delta)-\rho\left(\Delta^{\prime}\right)$ satisfies $\left.U \in M_{r}\left(\zeta \cdot B \llbracket z, z^{-1}\right\}\left[\frac{1}{t_{-}}\right]\right)$and $U=\rho(b)$. $\sigma^{*}(U) \cdot \rho\left(A^{-1}\right)$. Because $B$ is separated with respect to the norm $|$.$| , it follows that U=0$ and $\Delta^{\prime}=\Delta$.

We can now define the period morphism as a morphism of $\breve{E}$-analytic spaces

$$
\breve{\pi}:\left(\breve{\mathcal{M}}_{\underline{\mathbb{G}}_{0}}^{\hat{Z}^{-1}}\right)^{\text {an }} \rightarrow \breve{\mathcal{H}}_{G, \hat{Z}}^{\text {an }}
$$

as follows. Let $S$ be an affinoid, strictly $\breve{E}$-analytic space and let $S \rightarrow\left(\breve{\mathcal{M}}_{\mathbb{G}_{0}}^{\hat{Z}^{-1}}\right)^{\text {an }}$ be a morphism of $\breve{E}$-analytic spaces. With it we have to associate a uniquely determined morphism $S \rightarrow \breve{\mathcal{H}}_{G, \hat{Z}}^{\text {an }}$. Then the period morphism is obtained by glueing when $S$ runs through an affinoid covering of $\left(\breve{\mathcal{M}}_{\mathbb{G}_{0}}^{\hat{Z}^{-1}}\right)^{\text {an }}$.

Before we proceed, we recall that an algebra $B$ over $\breve{R}_{\hat{Z}}=\mathbb{F} \llbracket \xi \rrbracket$ is admissible in the

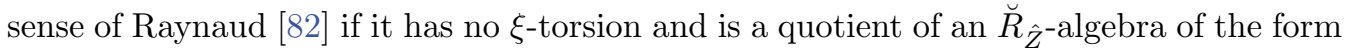

$$
\breve{R}_{\hat{Z}}\left\langle X_{1}, \ldots, X_{s}\right\rangle:=\left\{\sum_{\underline{n} \in \mathbb{N}_{0}^{s}} b_{\underline{n}} X_{1}^{n_{1}} \cdot \ldots \cdot X_{s}^{n_{s}}: b_{\underline{n}} \in \breve{R}_{\hat{Z}}, \lim _{|\underline{n}| \rightarrow \infty} b_{\underline{n}}=0\right\},
$$

where $\underline{n}=\left(n_{1}, \ldots, n_{s}\right)$ and $|\underline{n}|:=n_{1}+\ldots+n_{s}$. A formal $\breve{R}_{\hat{Z}}$-scheme $\mathscr{S}$ is admissible if it

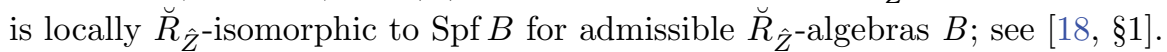

Recall that $\left(\breve{\mathcal{M}}_{\mathbb{G}_{0}}^{\hat{Z}^{-1}}\right)$ an is constructed as the union of the strictly $\breve{E}$-analytic spaces

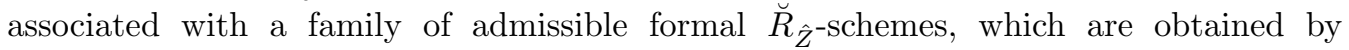
admissible formal blowing-ups of $\breve{\mathcal{M}}_{\mathbb{\mathbb { G }}_{0}}^{\hat{Z}^{-1}}$ in closed ideals; see [80, Chapter 5] or [13, §0.2]. By Raynaud's theorem, the morphism $S \rightarrow\left(\breve{\mathcal{M}}_{\mathbb{G}_{0}}^{\hat{Z}^{-1}}\right)^{\text {an }}$ is induced by a morphism from a

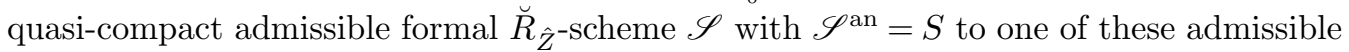

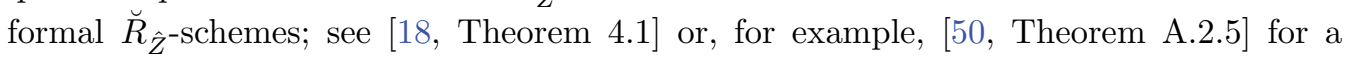


formulation with Berkovich spaces. Composing with the map to $\breve{\mathcal{M}}_{\mathbb{\mathbb { G }}_{0}}^{\hat{Z}^{-1}}$ yields a morphism of formal schemes $\mathscr{S} \rightarrow \breve{\mathcal{M}}_{\mathbb{\mathbb { G }}_{0}}^{\hat{Z}^{-1}}$. The latter corresponds to $(\underline{\mathcal{G}}, \bar{\delta}) \in \overline{\mathcal{M}}_{\underline{\mathbb{G}}_{0}}^{\hat{Z}^{-1}}(\mathscr{S})$.

Lemma 6.2. There is an étale covering $\mathscr{S}^{\prime}=\operatorname{Spf} B^{\prime} \rightarrow \mathscr{S}$ of admissible formal $\breve{R}_{\hat{Z}^{-}}$ schemes such that there is a trivialisation $\alpha: \underline{\mathcal{G}}_{\mathscr{S}^{\prime}} \stackrel{\sim}{\longrightarrow}\left(\left(L^{+} G\right)_{\mathscr{S}^{\prime}}, A \sigma^{*}\right)$ for some $A \in$ $G\left(B^{\prime} \llbracket z \rrbracket\left[\frac{1}{z-\zeta}\right]\right)$.

Proof. We may choose an étale covering $\overline{\mathscr{S}}^{\prime}$ of $\overline{\mathscr{S}}:=\mathrm{V} \mathscr{S}(\zeta)$ together with a trivialisation $\bar{\alpha}: \mathcal{G}_{\overline{\mathscr{S}}^{\prime}} \stackrel{\sim}{\longrightarrow}\left(L^{+} G\right)_{\overline{\mathscr{S}}^{\prime}}$. After refining the covering $\overline{\mathscr{S}}^{\prime}$, there is by [19, Lemma 1.4(a)] an

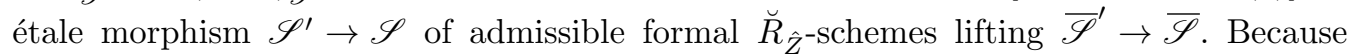
$\mathscr{S}$ is quasi-compact, we may further assume that $\mathscr{S}^{\prime}=\operatorname{Spf} B^{\prime}$ is affine. By [56, Proposition 2.2(c)], there is a lift $\alpha: \mathcal{G}_{\mathscr{S}^{\prime}} \stackrel{\sim}{\longrightarrow}\left(L^{+} G\right)_{\mathscr{S}^{\prime}}$ of the trivialisation $\bar{\alpha}$. Because $\underline{\mathcal{G}}$ is bounded by $\hat{Z}^{-1}$, this lift induces an isomorphism $\alpha: \underline{\mathcal{G}}_{\mathscr{S}^{\prime}} \stackrel{\sim}{\longrightarrow}\left(\left(L^{+} G\right)_{\mathscr{S}^{\prime}}, A \sigma^{*}\right)$ for $\bar{A} \in G\left(B^{\prime} \llbracket z \rrbracket\left[\frac{1}{z-\zeta}\right]\right)$; compare the proof of Proposition 2.6(a).

In addition, the quasi-isogeny $\bar{\delta}: \underline{\mathcal{G}}_{\overline{\mathscr{S}}^{\prime}} \rightarrow \underline{\mathbb{G}}_{0, \overline{\mathscr{S}}^{\prime}}$ corresponds under $\bar{\alpha}$ to an element $\bar{\Delta} \in L G\left(\bar{B}^{\prime}\right)$. We apply Lemma 6.1 to obtain a uniquely determined element $\Delta \in$ $\left.G\left(B^{\prime} \llbracket z, z^{-1}\right\}\left[\frac{1}{t_{-}}\right]\right)$lifting $\bar{\Delta}$ with $\Delta A=b \sigma^{*}(\Delta)$ for $A$ as in Lemma 6.2. We set

$$
\begin{aligned}
\gamma & :=\sigma^{*}(\Delta) A^{-1} \cdot G\left(B^{\prime}\left[\frac{1}{\zeta}\right] \llbracket z-\zeta \rrbracket\right)=b^{-1} \Delta \cdot G\left(B^{\prime}\left[\frac{1}{\zeta}\right] \llbracket z-\zeta \rrbracket\right) \\
& \in G\left(B^{\prime}\left[\frac{1}{\zeta}\right]((z-\zeta))\right) / G\left(B^{\prime}\left[\frac{1}{\zeta}\right] \llbracket z-\zeta \rrbracket\right)
\end{aligned}
$$

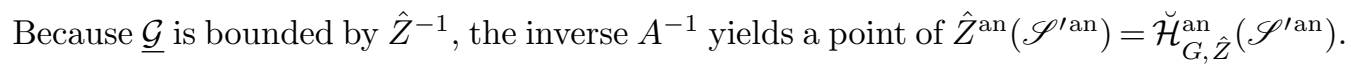
Because $\left.\sigma^{*}(\Delta) \in G\left(B^{\prime} \llbracket z, z^{-1}\right\}\left[\frac{1}{\sigma^{*} t_{-}}\right]\right)$and $\sigma^{*} t_{-} \in B^{\prime}\left[\frac{1}{\zeta}\right] \llbracket z-\zeta \rrbracket^{\times}$, we have

$$
\gamma \in G\left(B^{\prime}\left[\frac{1}{\zeta}\right] \llbracket z-\zeta \rrbracket\right) \cdot \breve{\mathcal{H}}_{G, \hat{Z}}^{\text {an }}=\breve{\mathcal{H}}_{G, \hat{Z}}^{\text {an }}
$$

using (6.2) and Definition 2.2(b)(v). If we replace our trivialisation $\alpha$ by a different trivialisation $\alpha^{\prime}: \underline{\mathcal{G}}_{\mathscr{S}^{\prime}} \stackrel{\sim}{\longrightarrow}\left(\left(L^{+} G\right)_{\mathscr{S}^{\prime}}, A^{\prime} \sigma^{*}\right)$, there is an $h \in L^{+} G\left(B^{\prime}\right)=G\left(B^{\prime} \llbracket z \rrbracket\right) \subset$ $G\left(B^{\prime}\left[\frac{1}{\zeta}\right] \llbracket z-\zeta \rrbracket\right)$ with $\alpha^{\prime}=h \cdot \alpha$ and $A^{\prime}=h A \sigma^{*}(h)^{-1}$. Then the quasi-isogeny $\bar{\delta}$ corresponds to $\bar{\Delta}^{\prime}=\bar{\Delta} h^{-1} \in L G\left(\bar{B}^{\prime}\right)$ and $\Delta^{\prime}=\Delta h^{-1}$ is the lift of $\bar{\Delta}^{\prime}$ from Lemma 6.1. This yields

$$
\gamma^{\prime}=b^{-1} \Delta^{\prime} \cdot G\left(B^{\prime}\left[\frac{1}{\zeta}\right] \llbracket z-\zeta \rrbracket\right)=b^{-1} \Delta \cdot G\left(B^{\prime}\left[\frac{1}{\zeta}\right] \llbracket z-\zeta \rrbracket\right)=\gamma .
$$

Therefore, $\gamma$ descends to an element $\gamma \in \breve{\mathcal{H}}_{G, \hat{Z}}^{\text {an }}(S)$ giving the desired morphism $S \rightarrow \breve{\mathcal{H}}_{G, \hat{Z}}^{\text {an }}$. This completes the construction of the period morphism.

Definition 6.3. The morphism $\breve{\pi}:\left(\breve{\mathcal{M}}_{\underline{\mathbb{G}}_{0}}^{\hat{Z}^{-1}}\right)^{\text {an }} \rightarrow \breve{\mathcal{H}}_{G, \hat{Z}}^{\text {an }}$ of $\breve{E}$-analytic spaces constructed above is called the period morphism associated with $\underline{\mathbb{G}}_{0}$ and $\hat{Z}$.

Remark 6.4. If $G=\mathrm{GL}_{r}$ and $B$ is an admissible $\mathbb{F} \llbracket \zeta \rrbracket$-algebra in the sense of Raynaud, there is an equivalence $[56, \S 4]$ between local $\mathrm{GL}_{r}$-shtukas and local shtukas $\underline{M}=\left(M, \tau_{M}\right)$ over $\mathscr{S}=\operatorname{Spf} B$ consisting of a locally free $B \llbracket z \rrbracket$-module $M$ of rank $r$ and an isomorphism $\tau_{M}: \sigma^{*} M\left[\frac{1}{z-\zeta}\right] \stackrel{\sim}{\longrightarrow} M\left[\frac{1}{z-\zeta}\right]$. The de Rham cohomology 


$$
\mathfrak{p}^{\underline{M}}:=\mathrm{H}_{\mathrm{dR}}^{1}\left(\underline{M}, B\left[\frac{1}{\zeta}\right] \llbracket z-\zeta \rrbracket\right):=\sigma^{*} M \otimes_{B \llbracket z \rrbracket} B\left[\frac{1}{\zeta}\right] \llbracket z-\zeta \rrbracket
$$

of $\underline{M}$ over $\mathscr{S}^{\text {an }}$ carries a natural Hodge-Pink structure

$$
\mathfrak{q}^{\underline{M}}:=\tau_{M}^{-1}\left(M \otimes_{B \llbracket z \rrbracket} B\left[\frac{1}{\zeta}\right] \llbracket z-\zeta \rrbracket\right) \subset \mathfrak{p}^{\underline{M}}\left[\frac{1}{z-\zeta}\right] ;
$$

see [54, Definition 3.4.13]. If, moreover, there is a fixed local $\mathrm{GL}_{r}$-shtuka $\underline{\mathbb{G}}_{0}$ over $\mathbb{F}$ with associated local shtuka $\left(\mathbb{M}, \tau_{\mathbb{M}}\right)=\left(\mathbb{F} \llbracket z \rrbracket^{r}, b \sigma^{*}\right)$ and a quasi-isogeny $\bar{\delta}: \underline{\mathcal{G}}_{\text {Spec } B /(\zeta)} \rightarrow$ $\underline{\mathbb{G}}_{0}$, Spec $B /(\zeta)$ given by $\bar{\Delta} \in L G(B /(\zeta))$, then the lift $\Delta$ from Lemma 6.1 provides an isomorphism

$$
\sigma^{*}(\Delta): \mathrm{H}_{\mathrm{dR}}^{1}\left(\underline{M}, B\left[\frac{1}{\zeta}\right] \llbracket z-\zeta \rrbracket\right) \stackrel{\sim}{\longrightarrow} \mathrm{H}_{\mathrm{cris}}^{1}(\underline{\mathbb{M}}, \mathbb{F}((z))) \otimes_{\mathbb{F}((z))} B\left[\frac{1}{\zeta}\right] \llbracket z-\zeta \rrbracket
$$

that transports the Hodge-Pink structure $\mathfrak{q}^{\underline{M}}$ to the family

$$
\sigma^{*}(\Delta) \circ \tau_{M}^{-1}\left(M \otimes_{B \llbracket z \rrbracket} B\left[\frac{1}{\zeta}\right] \llbracket z-\zeta \rrbracket\right)
$$

of Hodge-Pink structures on the constant $z$-isocrystal $\mathrm{H}_{\text {cris }}^{1}(\underline{\mathbb{M}}, \mathbb{F}((z))):=\sigma^{*} \underline{\mathbb{M}} \otimes_{\mathbb{F} \llbracket z \rrbracket} \mathbb{F}((z))$; see [54, Definition 3.5.14]. Our period morphism $\breve{\pi}$ associates this family of Hodge-Pink structures with the universal local $\mathrm{GL}_{r}$-shtuka over $\breve{\mathcal{M}}_{\mathbb{G}_{0}}^{\hat{Z}^{-1}}$. More precisely, this family equals $\gamma \cdot B\left[\frac{1}{\zeta}\right] \llbracket z-\zeta \rrbracket^{r}$ where the element $\gamma$ from (6.4) is the image under $\breve{\pi}$ of the local $\mathrm{GL}_{r}$-shtuka $(\underline{M}, \bar{\delta}) \in \breve{\mathcal{M}}_{\underline{\underline{G}}_{0}}^{\hat{Z}^{-1}}(\operatorname{Spf} B)$.

Remark 6.5. The period morphism $\breve{\pi}$ is equivariant for the action of $J:=J_{b}\left(\mathbb{F}_{q}((z))\right)$. Indeed, $j \in J$ acts on $\breve{\mathcal{M}}_{\mathbb{G}_{0}}^{\hat{Z}^{-1}}$ by $j:(\underline{\mathcal{G}}, \bar{\delta}) \mapsto(\underline{\mathcal{G}}, j \circ \bar{\delta})$. In terms of (6.4) this means that $j \in J \subset G(\mathbb{F}((z)))$ sends $\Delta$ to $j \cdot \Delta$ and $\gamma$ to $\sigma^{*}(j) \cdot \gamma$. Thus, it coincides with the action on $\breve{\mathcal{H}}_{G, \hat{Z}}^{\text {an }}$ defined in Remark 4.17.

Remark 6.6. As in the arithmetic case, these period morphisms are not compatible with Weil descent data of source and target. Here the source is equipped with the Weil descent datum induced by the one in Remark 3.3. On the target we have the natural Weil descent datum given by the fact that $\mathcal{H}_{G, \hat{Z}}^{\text {an }}$ is defined over $E_{\hat{Z}}$. In order to ensure such a compatibility, one has to extend the period morphism by a second component mapping to $\pi_{1}(G)_{\Gamma}$. For a more detailed discussion we refer to [80] or [79, Properties 4.27(iv)].

Remark 6.7. In the above construction the bounded local $G$-shtuka $\left(\left(L^{+} G\right)_{\mathscr{S}^{\prime}}, A \sigma^{*}\right)$ over $\mathscr{S}^{\prime}$ with $A \in G\left(B^{\prime} \llbracket z \rrbracket\left[\frac{1}{z-\zeta}\right]\right)$ induces an étale local $G$-shtuka $\left(\left(L^{+} G\right)_{S^{\prime}}, A \sigma^{*}\right)$ over $S^{\prime}:=\left(\mathscr{S}^{\prime}\right)^{\text {an }}$ in the sense of Definition 6.8, because $(z-\zeta)^{-1}=-\sum_{i=0}^{\infty} \zeta^{-i-1} z^{i} \in$ $\mathcal{O}_{S^{\prime}}\left(S^{\prime}\right) \llbracket z \rrbracket$ implies $B^{\prime} \llbracket z \rrbracket\left[\frac{1}{z-\zeta}\right] \subset \mathcal{O}_{S^{\prime}}\left(S^{\prime}\right) \llbracket z \rrbracket$. The isomorphism $\alpha: \underline{\mathcal{G}}_{\mathscr{S}^{\prime}} \stackrel{\sim}{\longrightarrow}\left(\left(L^{+} G\right)_{\mathscr{S}^{\prime}}, A \sigma^{*}\right)$ yields a descent datum $g:=p r_{2}^{*} \alpha \circ p r_{1}^{*} \alpha^{-1} \in L^{+} G\left(\mathscr{S}^{\prime \prime}\right)$ with $p r_{2}^{*} A \cdot \sigma^{*}(g)=g \cdot p r_{1}^{*} A$, where $\mathscr{S}^{\prime \prime}:=\mathscr{S}^{\prime} \times \mathscr{S} \mathscr{S}^{\prime}$ and $p r_{i}: \mathscr{S}^{\prime \prime} \rightarrow \mathscr{S}^{\prime}$ is the projection onto the $i$ th factor. Viewing $g \in L^{+} G\left(S^{\prime \prime}\right)$ where $S^{\prime \prime}:=\left(\mathscr{S}^{\prime \prime}\right)^{\text {an }}=S^{\prime} \times{ }_{S} S^{\prime}$ provides a descent datum $p r_{1}^{*}\left(L^{+} G\right)_{S^{\prime}} \stackrel{\sim}{\longrightarrow} p r_{2}^{*}\left(L^{+} G\right)_{S^{\prime}}$ on the $L^{+} G$-torsor $\left(L^{+} G\right)_{S^{\prime}}$ via multiplication by $g$ on the right. This allows to descend $\left(\left(L^{+} G\right)_{S^{\prime}}, A \sigma^{*}\right)$ to an étale local $G$-shtuka over $S=\mathscr{S}^{\text {an }}$, which by abuse of notation we denote again by $\underline{\mathcal{G}}$. In this way we obtain the universal family of étale local $G$-shtukas over $\left(\breve{\mathcal{M}}_{\mathbb{G}_{0}}^{\hat{Z}^{-1}}\right)^{\text {an }}$. 
Definition 6.8. Let $S$ be an $\mathbb{F}((\zeta))$-scheme or a strictly $\mathbb{F}((\zeta))$-analytic space. An étale local $G$-shtuka over $S$ is a pair $\underline{\mathcal{G}}=\left(\mathcal{G}, \tau_{\mathcal{G}}\right)$ consisting of an $L^{+} G$-torsor $\mathcal{G}$ on $S$ and an isomorphism $\tau_{\mathcal{G}}: \sigma^{*} \mathcal{G} \stackrel{\sim}{\longrightarrow}$ of $L^{+} G$-torsors.

Proposition 6.9. The period morphism factors through the open $\breve{E}$-analytic subspace $\breve{\mathcal{H}}_{G, \hat{Z}, b}^{a}$.

Proof. Let $x$ be a point of $\left(\breve{\mathcal{M}}_{\mathbb{\mathbb { G }}_{0}}^{\hat{Z}^{-1}}\right)$ an with values in a complete field extension $L$ of $\breve{E}$ and let $\gamma:=\breve{\pi}(x) \in \breve{\mathcal{H}}_{G, \hat{Z}}^{\text {an }}$ be its image under the period morphism. Then $x$ corresponds to a pair $(\underline{\mathcal{G}}, \bar{\delta}) \in \breve{\mathcal{M}}_{\underline{\mathbb{G}}_{0}}^{\hat{Z}^{-1}}\left(\operatorname{Spf} \mathcal{O}_{L}\right)$ where $\underline{\mathcal{G}}$ is a local $G$-shtuka over the valuation ring $\mathcal{O}_{L}$ of $L$. Choose a faithful $\mathbb{F}_{q} \llbracket z \rrbracket$-rational representation $(\rho, V)$ of $G$, and under the equivalence between local GL $(V)$-shtukas and local shtukas from Remark 6.4 let $\underline{M}:=M\left(\rho_{*} \underline{\mathcal{G}}\right)$ be the associated local shtuka over $\mathcal{O}_{L}$, and let $M\left(\rho_{*} \bar{\delta}\right)$ be the associated quasi-isogeny. By [50, Proposition 2.4.4] the $\sigma$-bundle $\underline{\mathcal{F}}_{b, \gamma}(V)$ is isomorphic to $\underline{M} \otimes_{\mathcal{O}_{L} \llbracket z \rrbracket} L\left\langle\frac{z}{\zeta^{s}}, z^{-1}\right\}$ and hence $\left.\underline{\mathcal{F}}_{b, \gamma}(V) \otimes_{L\left\langle\frac{z}{\zeta^{s}}\right.}, z^{-1}\right\} \bar{L}\left\langle\frac{z}{\zeta^{s}}, z^{-1}\right\} \cong \underline{\mathcal{F}}_{0,1} \oplus \operatorname{dim} \rho$; see [50, (Proof of) Theorem 2.4.7]. In other words, $\gamma \in \breve{\mathcal{H}}_{G, \hat{Z}, b}^{a}$.

Proposition 6.10. The period morphism $\breve{\pi}:\left(\breve{\mathcal{M}}_{\mathbb{G}_{0}}^{\hat{Z}^{-1}}\right)^{a n} \rightarrow \breve{\mathcal{H}}_{G, \hat{Z}, b}^{a}$ is étale.

For later use, we formulate one smaller step in the proof as a separate lemma.

Lemma 6.11. $\left(\breve{\mathcal{M}}_{\underline{\mathbb{G}}_{0}}^{\hat{Z}^{-1}}\right)^{\text {ad }}$ is separated and partially proper over $\breve{E}$.

Proof. The irreducible components of its special fibre are proper by Theorem 3.5. Thus, the lemma follows from [63, Remark 1.3.18].

Proof of Proposition 6.10. Let $\breve{\pi}^{\text {rig }}:\left(\breve{\mathcal{M}}_{\mathbb{G}_{0}}^{\hat{Z}^{-1}}\right)^{\text {rig }} \rightarrow\left(\breve{\mathcal{H}}_{G, \hat{Z}, b}^{a}\right)^{\text {rig }}$ be the associated morphism of rigid analytic spaces and let $\breve{\pi}^{\text {ad }}:\left(\breve{\mathcal{M}}_{\mathbb{G}_{0}}^{\hat{Z}^{-1}}\right)^{\text {ad }} \rightarrow\left(\breve{\mathcal{H}}_{G, \hat{Z}, b}^{a}\right)^{\text {ad }}$ be the associated morphism of adic spaces in the sense of Huber [63]. By [63, Assertion (a) on p. 427], the morphism $\breve{\pi}$ is étale if and only if $\breve{\pi}^{\text {ad }}$ is étale and partially proper. The subspace $\left(\breve{\mathcal{H}}_{G, \hat{Z}, b}^{a}\right)^{\text {ad }} \subset\left(\breve{\mathcal{H}}_{G, \hat{Z}}\right)^{\text {ad }}$ is open by Theorem 4.20 and [63, Assertion (1) on p. 431]. Therefore, $\left(\breve{\mathcal{H}}_{G, \hat{Z}, b}^{a}\right)^{\text {ad }}$ is separated over $\breve{E}$ by [63, Lemma 1.10.17]. So by [63, Lemma 1.10.17(vi)] and the above lemma, $\breve{\pi}^{\text {ad }}$ is partially proper.

It remains to show that $\breve{\pi}^{\text {ad }}$ is étale. By [63, Proposition 1.7.11], this is equivalent to $\breve{\pi}^{\text {rig }}$ being étale. So by [21, Proposition 2.4] we must show that for any admissible $\breve{R}_{\hat{Z}^{\text {-algebra }}}$ $B$ in the sense of Raynaud and for any ideal $I \subset B$ with $I^{2}=0$ and any commutative diagram with solid arrows

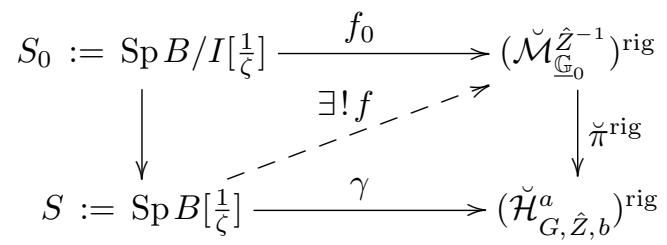


there is a unique dashed arrow $f$ making the diagram commutative. We set $\mathscr{S}:=\operatorname{Spf} B$, as well as $B_{0}:=B / I$ and $\mathscr{S}_{0}:=\operatorname{Spf} B_{0}$. We will construct $f$ as a morphism $\mathscr{S} \rightarrow \mathcal{M}_{\mathbb{G}_{0}}^{\hat{Z}^{-1}}$ after replacing $\mathscr{S}$ by an admissible blowing-up. Note that every admissible blowing-up of $\mathscr{S}_{0}$ is induced by an admissible blowing-up of $\mathscr{S}$. Moreover, in the course of the proof we may replace $S$ by a quasi-compact, quasi-separated, étale covering $S^{\prime} \rightarrow S$. Namely, by [19, Corollaries 5.10 and 5.4], every such covering is obtained from a quasi-compact morphism $\mathscr{S}^{\prime} \rightarrow \mathscr{S}$ of formal schemes that is faithfully flat after replacing $\mathscr{S}$ by an admissible blowing-up. By the uniqueness assertion for $f$ it suffices to construct $f$ over $\mathscr{S}^{\prime}$ and descend it back to $\mathscr{S}$.

After replacing $\mathscr{S}$ by an admissible blowing-up, the morphism $f_{0}$ extends to $f_{0}: \mathscr{S}_{0} \rightarrow$ $\breve{\mathcal{M}}_{\mathbb{G}_{0}}^{\hat{Z}^{-1}}$ and corresponds to a pair $\left(\underline{\mathcal{G}}_{0}, \bar{\delta}_{0}\right) \in \breve{\mathcal{M}}_{\mathbb{G}_{0}}^{\hat{Z}^{-1}}\left(\mathscr{S}_{0}\right)$ where $\underline{\mathcal{G}}_{0}$ is a local $G$-shtuka over $\mathscr{S}_{0}$. By Lemma 6.2 we may replace $\mathscr{S}_{0}$ by an étale covering such that $\underline{\mathcal{G}}_{0} \cong$ $\left(\left(L^{+} G\right)_{\mathscr{S}_{0}}, A_{0} \sigma^{*}\right)$ for some $A_{0} \in G\left(B_{0} \llbracket z \rrbracket\left[\frac{1}{z-\zeta}\right]\right)$. By [45, Théorème I.8.3], this étale covering lifts uniquely to an étale covering of $\mathscr{S}$. The quasi-isogeny $\bar{\delta}_{0}: \underline{\mathcal{G}}_{0, \bar{S}_{0}} \rightarrow \underline{\mathbb{G}}_{0, \overline{\mathscr{S}}_{0}}$ over $\overline{\mathscr{S}}_{0}:=\operatorname{Spec} B_{0} /(\zeta)$ corresponds to an element $\bar{\Delta}_{0} \in L G\left(B_{0} /(\zeta)\right)$ that lifts by Lemma 6.1 to a unique $\left.\Delta_{0} \in G\left(B_{0} \llbracket z, z^{-1}\right\}\left[\frac{1}{t_{-}}\right]\right)$with $\Delta_{0} \bmod \zeta=\bar{\Delta}_{0}$ and $\Delta_{0} \cdot A_{0}=$ $b \cdot \sigma^{*}\left(\Delta_{0}\right)$. If we let $\gamma_{0} \in \breve{\mathcal{H}}_{G, \hat{z}, b}^{a}\left(S_{0}\right)$ be the pullback of $\gamma \in \breve{\mathcal{H}}_{G, \hat{Z}, b}^{a}(S)$, then $\gamma_{0}=$ $\sigma^{*}\left(\Delta_{0}\right) A_{0}^{-1} \cdot G\left(B_{0}\left[\frac{1}{\zeta}\right] \llbracket z-\zeta \rrbracket\right)$ and $\sigma^{*}\left(\Delta_{0}\right)^{-1} \gamma_{0}=A_{0}^{-1} \cdot G\left(B_{0}\left[\frac{1}{\zeta}\right] \llbracket z-\zeta \rrbracket\right)$.

We claim that $\sigma^{*}\left(\Delta_{0}\right)$ lifts to a uniquely determined element of $\left.G\left(B \llbracket z, z^{-1}\right\}\left[\frac{1}{\sigma^{*} t_{-}}\right]\right)$. Indeed, after choosing a faithful $\mathbb{F}_{q} \llbracket z \rrbracket$-rational representation $\rho: G \hookrightarrow \mathrm{SL}_{r}$ there is an integer $e$ such that the matrix $\left.t_{-}^{e} \cdot \rho\left(\Delta_{0}\right) \in B_{0} \llbracket z, z^{-1}\right\}^{r \times r}$. Because $\sigma^{*}(I)=0$, the morphism $\sigma^{*}: B \rightarrow B, x \mapsto x^{q}$ factors over $B \rightarrow B_{0} \rightarrow B$, and so $\left(\sigma^{*} t_{-}\right)^{e} \cdot \rho\left(\sigma^{*} \Delta_{0}\right)=$ $\left.\sigma^{*}\left(t_{-}^{e} \cdot \rho\left(\Delta_{0}\right)\right) \in B \llbracket z, z^{-1}\right\}^{r \times r}$. This implies $\left.\rho\left(\sigma^{*} \Delta_{0}\right) \in \operatorname{SL}_{r}\left(B \llbracket z, z^{-1}\right\}\left[\frac{1}{\sigma^{*} t_{-}}\right]\right)$and $\sigma^{*}\left(\Delta_{0}\right) \in$ $\left.G\left(B \llbracket z, z^{-1}\right\}\left[\frac{1}{\sigma^{*} t_{-}}\right]\right)$. By $(6.2)$ it follows, moreover, that $\sigma^{*}\left(\Delta_{0}\right) \in G\left(B\left[\frac{1}{\zeta}\right] \llbracket z-\zeta \rrbracket\right)$.

We now replace $S$ by a quasi-compact, quasi-separated, étale covering over which $\sigma^{*}\left(\Delta_{0}\right)^{-1} \gamma$ is induced from an element $g \in G\left(B\left[\frac{1}{\zeta}\right]((z-\zeta))\right)$. Consider the element $c_{0}:=(g \bmod I)^{-1} A_{0}^{-1} \in G\left(B_{0}\left[\frac{1}{\zeta}\right] \llbracket z-\zeta \rrbracket\right)$. Because the kernel of $B\left[\frac{1}{\zeta}\right] \llbracket z-\zeta \rrbracket \rightarrow B_{0}\left[\frac{1}{\zeta}\right] \llbracket z-\zeta \rrbracket$ is a nilpotent ideal and $G$ is smooth, $c_{0}$ lifts to an element $c \in G\left(B\left[\frac{1}{\zeta}\right] \llbracket z-\zeta \rrbracket\right)$ and replacing $g$ by $g c$ yields $g \bmod I=A_{0}^{-1}$. Also, $\sigma^{*}\left(\Delta_{0}\right)^{-1} \gamma \in\left(\breve{\mathcal{H}}_{G, \hat{Z}}\right)^{\text {rig }}(S)$ by Definition 2.2(b)(v), whence $g \cdot G\left(B\left[\frac{1}{\zeta}\right] \llbracket z-\zeta \rrbracket\right) \in\left(\hat{Z}_{E} \widehat{\otimes}_{E} \breve{E}\right)^{\mathrm{rig}}(S)$. We denote the corresponding morphism of rigid analytic spaces by $\alpha: S \rightarrow\left(\hat{Z}_{E} \widehat{\otimes}_{E} \breve{E}\right)^{\text {rig }}$. Let $R$ be an extension of $R_{\hat{Z}}$ over which a representative $\hat{Z}_{R}$ of $\hat{Z}$ exists and such that $\operatorname{Frac}(R)$ is a finite Galois extension of $E_{\hat{Z}}$. We let $\breve{R}$ be the ring of integers in the completion of the maximal unramified extension of $\operatorname{Frac}(R)$, and we set $\hat{Z}_{\breve{R}}:=\hat{Z}_{R} \widehat{\times}_{R} \operatorname{Spf} \breve{R}$. By applying Galois descent with respect to the field extension $\operatorname{Frac}(\breve{R}) / \breve{E}$ in the end, we may restrict to the case where $\mathscr{S}$ is a formal scheme over $\operatorname{Spf} \breve{R}$ and not just over $\operatorname{Spf} \breve{R}_{\hat{Z}}$. Let $\mathscr{S}^{\prime} \subset \hat{Z}_{\breve{R}} \widehat{\times}_{\breve{R}} \mathscr{S}$ be the $\zeta$-adic completion of the scheme theoretic closure of the graph of $\alpha$. It is projective over $\mathscr{S}$ by Proposition 2.6(d) and therefore $\left(\mathscr{S}^{\prime}\right)^{\text {rig }}=\mathscr{S}^{\text {rig }}$. So $\mathscr{S}^{\prime} \rightarrow \mathscr{S}$ is an admissible blowing-up by [19, Corollary 5.4] and we replace $\mathscr{S}$ by $\mathscr{S}^{\prime}$ to obtain an extension $\alpha: \mathscr{S} \rightarrow \hat{Z}_{\breve{R}}$. After replacing $\mathscr{S}$ by an étale covering, this morphism $\alpha$ is of the form $A^{-1} \cdot G(B \llbracket z \rrbracket) \in \hat{Z}_{\breve{R}}(\mathscr{S})$ for an element $A^{-1} \in G\left(B \llbracket z \rrbracket\left[\frac{1}{z-\zeta}\right]\right)$; compare the proof of 
Proposition 2.6. Because $g$ and $A^{-1}$ both correspond to the morphism $\alpha: S \rightarrow\left(\breve{\mathcal{H}}_{G, \hat{z}}\right)^{\text {rig }}$, we have $g \cdot G\left(B\left[\frac{1}{\zeta}\right] \llbracket z-\zeta \rrbracket\right)=A^{-1} \cdot G\left(B\left[\frac{1}{\zeta}\right] \llbracket z-\zeta \rrbracket\right)$ in $\left(\breve{\mathcal{H}}_{G, \hat{Z}}\right)^{\text {rig }}(S)$. We consider the element $a_{0}:=A_{0}\left(A^{-1} \bmod I\right) \in G\left(B_{0} \llbracket z \rrbracket\left[\frac{1}{z-\zeta}\right]\right)$. Its image in $\left(\breve{\mathcal{H}}_{G, \hat{Z}}\right)^{\text {rig }}\left(S_{0}\right)$ equals

$$
\begin{aligned}
\left(g^{-1} \bmod I\right)\left(A^{-1} \bmod I\right) \cdot G\left(B_{0}\left[\frac{1}{\zeta}\right] \llbracket z-\zeta \rrbracket\right) & =\left(g^{-1} g \bmod I\right) \cdot G\left(B_{0}\left[\frac{1}{\zeta}\right] \llbracket z-\zeta \rrbracket\right) \\
& =1 \cdot G\left(B_{0}\left[\frac{1}{\zeta}\right] \llbracket z-\zeta \rrbracket\right) ;
\end{aligned}
$$

that is, $a_{0} \in G\left(B_{0}\left[\frac{1}{\zeta}\right] \llbracket z-\zeta \rrbracket\right)$. By the following Lemma 6.13, this implies that $a_{0} \in$ $G\left(B_{0} \llbracket z \rrbracket\right)$. Again, because the kernel of $B \llbracket z \rrbracket \rightarrow B_{0} \llbracket z \rrbracket$ is nilpotent and $G$ is smooth, $a_{0}$ lifts to an element $a \in G(B \llbracket z \rrbracket)$, and replacing $A^{-1}$ by $A^{-1} a^{-1}$ yields $A \bmod I=$ $A_{0}$. We consider the local $G$-shtuka $\underline{\mathcal{G}}:=\left(\left(L^{+} G\right)_{\mathscr{S}}, A \sigma^{*}\right)$ with $\underline{\mathcal{G}}_{\mathscr{S}_{0}}=\underline{\mathcal{G}}_{0}$. Then $\Delta_{0}$ lifts to $\left.\Delta:=b \sigma^{*}\left(\Delta_{0}\right) A^{-1} \in G\left(B \llbracket z, z^{-1}\right\}\left[\frac{1}{t_{-}}\right]\right)$and $\bar{\Delta}:=\Delta \bmod (\zeta): \underline{\mathcal{G}}_{\bar{S}} \rightarrow \underline{\mathbb{G}}_{0, \bar{S}}$ is the unique lift of the quasi-isogeny $\bar{\delta}_{0}=\bar{\Delta}_{0}$ by rigidity. We let $f: \mathscr{S} \rightarrow \breve{\mathcal{M}}_{\mathbb{G}_{0}}^{\hat{Z}^{-1}}$ be the morphism given by $(\underline{\mathcal{G}}, \bar{\Delta}) \in \breve{\mathcal{M}}_{\underline{\mathbb{G}}_{0}}^{\hat{Z}^{-1}}(\mathscr{S})$. It makes the diagram (6.5) commutative, because $\sigma^{*}(\Delta) A^{-1} \cdot G\left(B\left[\frac{1}{\zeta}\right] \llbracket z-\zeta \rrbracket\right)=\bar{\sigma}^{*}\left(\Delta_{0}\right) g \cdot G\left(B\left[\frac{1}{\zeta}\right] \llbracket z-\zeta \rrbracket\right)=\gamma$.

To prove that $f$ is uniquely determined, let $f^{\prime}: \mathscr{S} \rightarrow \breve{\mathcal{M}}_{\mathbb{G}_{0}}^{\hat{Z}^{-1}}$ be a second morphism making the diagram (6.5) commutative. The corresponding point $\left(\underline{\mathcal{G}}^{\prime}, \bar{\delta}^{\prime}\right) \in \breve{\mathcal{M}}_{\mathbb{\mathbb { G }}_{0}}^{\hat{Z}^{-1}}(\mathscr{S})$ is of the form $\underline{\mathcal{G}}^{\prime}=\left(\left(L^{+} G\right)_{\mathscr{S}}, A^{\prime} \sigma^{*}\right)$ with $A^{\prime} \bmod I=A_{0}$ and $\Delta^{\prime}=b \sigma^{*}\left(\Delta_{0}\right) A^{\prime-1} \in$ $\left.G\left(B \llbracket z, z^{-1}\right\}\left[\frac{1}{t_{-}}\right]\right)$. We assume that it is mapped under $\breve{\pi}^{\text {rig }}$ also to $\gamma$. This means $\sigma^{*}\left(\Delta_{0}\right) A^{-1} \cdot G\left(B\left[\frac{1}{\zeta}\right] \llbracket z-\zeta \rrbracket\right)=\gamma=\sigma^{*}\left(\Delta_{0}\right) A^{\prime-1} \cdot G\left(B\left[\frac{1}{\zeta}\right] \llbracket z-\zeta \rrbracket\right)$, whence $\Phi:=A^{\prime} A^{-1} \in$ $G\left(B\left[\frac{1}{\zeta}\right] \llbracket z-\zeta \rrbracket\right) \subset G\left(B\left[\frac{1}{\zeta}\right]((z-\zeta))\right)$. From Lemma 6.13 it follows that $\Phi \in G(B \llbracket z \rrbracket)$. Also, $\sigma^{*}(\Phi)=\sigma^{*}\left(A^{\prime} A^{-1} \bmod I\right)=\sigma^{*}(1)=1$ implies $\Phi A=A^{\prime} \sigma^{*}(\Phi)$ and $\Phi=\Delta^{\prime-1} \Delta$. We conclude that $\Phi$ is an isomorphism $(\underline{\mathcal{G}}, \bar{\delta}) \stackrel{\sim}{\sim}\left(\underline{\mathcal{G}}^{\prime}, \bar{\delta}^{\prime}\right)$. This means $f=f^{\prime}$ and finishes the proof.

Remark 6.12. When $G=\mathrm{GL}_{r}$, the proof starts in terms of Remark 6.4 with a local shtuka $\underline{M}_{0}$ over $\mathscr{S}_{0}$. Then it considers the de Rham cohomology of $\underline{M}_{0}$, which lifts to $\mathscr{S}$ by its crystalline nature. Next it produces from the Hodge-Pink structure $\gamma$ a Hodge-Pink structure on the de Rham cohomology of $\underline{M}_{0}$ over $\mathscr{S}$ that lifts the Hodge-Pink structure of $\underline{M}_{0}$. This lift of the Hodge-Pink structure corresponds to a unique lift of $\underline{M}_{0}$ to a local shtuka $\underline{M}$ over $\mathscr{S}$ by [41, Proposition 6.3]. In that sense, our proof is a direct translation of [80, Proposition 5.17].

Lemma 6.13. Let $B$ be an $\mathbb{F}_{q} \llbracket \zeta \rrbracket$-algebra without $\zeta$-torsion that is $\zeta$-adically complete, and let $a \in G\left(B \llbracket z \rrbracket\left[\frac{1}{z-\zeta}\right]\right)$ such that the image of a in $G\left(B\left[\frac{1}{\zeta}\right]((z-\zeta))\right)$ lies in $G\left(B\left[\frac{1}{\zeta}\right] \llbracket z-\zeta \rrbracket\right)$. Then $a \in G(B \llbracket z \rrbracket)$.

Proof. Note that $B \llbracket z \rrbracket$ has no $(z-\zeta)$-torsion, because $B$ has no $\zeta$-torsion. Let $\rho: G \hookrightarrow \mathrm{SL}_{r}$ be a faithful representation over $\mathbb{F}_{q} \llbracket z \rrbracket$ and consider the matrix $\rho(a) \in \mathrm{SL}_{r}\left(B \llbracket z \rrbracket\left[\frac{1}{z-\zeta}\right]\right) \subset$ $B \llbracket z \rrbracket\left[\frac{1}{z-\zeta}\right]^{r \times r}$.

It is enough to show that this matrix is in $B \llbracket z \rrbracket^{r \times r}$ as $\mathrm{SL}_{r}\left(B \llbracket z \rrbracket\left[\frac{1}{z-\zeta}\right]\right) \cap B \llbracket z \rrbracket^{r \times r}=$ $\mathrm{SL}_{r}(B \llbracket z \rrbracket)$ and $\mathrm{SL}_{r}(B \llbracket z \rrbracket) \cap \rho\left(G\left(B \llbracket z \rrbracket\left[\frac{1}{z-\zeta}\right]\right)\right)=\rho(G(B \llbracket z \rrbracket))$ as $\rho$ is defined over $\mathbb{F}_{q} \llbracket z \rrbracket$. 
After multiplying $\rho(a)$ by $(z-\zeta)^{n}$ for sufficiently large $n$, its denominators disappear and its image in $B\left[\frac{1}{\zeta}\right] \llbracket z-\zeta \rrbracket^{r \times r}$ is divisible by $(z-\zeta)^{n}$. Thus, it suffices to show that an element $f$ of $B \llbracket z \rrbracket$ whose image in $B\left[\frac{1}{\zeta}\right] \llbracket z-\zeta \rrbracket$ is divisible by $z-\zeta$ is already divisible by $z-\zeta$ in $B \llbracket z \rrbracket$. This follows as in Lemma 7.6.

We end this section with some examples.

Example 6.14 (The Drinfeld period morphism.). This example is due to Drinfeld [35]. A good account is given by Genestier and Lafforgue [39], [40]. Let $d$ be a positive integer and let $D$ be the central division algebra over $\mathbb{F}_{q}((z))$ of Hasse invariant $1 / d$. Let $\mathcal{O}_{D}$ be its maximal order. We may identify $D \cong \bigoplus_{i=0}^{d-1} \mathbb{F}_{q^{d}}((z)) \Pi^{i}$ and $\mathcal{O}_{D} \cong \bigoplus_{i=0}^{d-1} \mathbb{F}_{q^{d}} \llbracket z \rrbracket \Pi^{i}$ with $\Pi^{d}=z$ and $\Pi a=\sigma(a) \Pi$ for $a \in \mathbb{F}_{q^{d}}((z))$. We let $G:=\mathcal{O}_{D}^{\times}$be the group scheme over $\mathbb{F}_{q} \llbracket z \rrbracket$ with $G(A)=\left(\mathcal{O}_{D} \otimes_{\mathbb{F}_{q} \llbracket z \rrbracket} A\right)^{\times}$for $\mathbb{F}_{q} \llbracket z \rrbracket$-algebras $A$. Consider the matrices

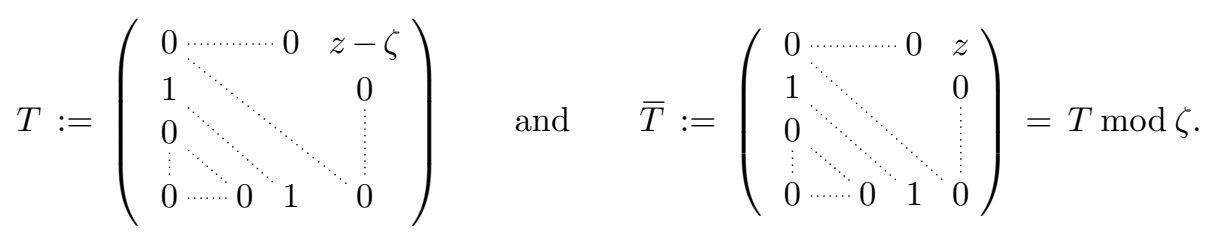

The field extension $\mathbb{F}_{q^{d}}$ of $\mathbb{F}_{q}$ splits the division algebra $D$ by the isomorphisms $D \otimes_{\mathbb{F}_{q}}$ $\mathbb{F}_{q^{d}} \cong \mathbb{F}_{q^{d}}((z))^{d \times d}$ and $\mathcal{O}_{D} \otimes_{\mathbb{F}_{q}} \mathbb{F}_{q^{d}} \cong\left\{g \in \mathbb{F}_{q^{d}} \llbracket z \rrbracket^{d \times d}: g \bmod z\right.$ islowertriangular $\}$ sending $\Pi \otimes 1$ to $\bar{T}$ and $a \otimes 1$ to $\operatorname{diag}\left(\sigma^{d-1}(a), \sigma^{d-2}(a), \ldots, a\right)$ for $a \in \mathbb{F}_{q^{d}}((z)) \subset D$. So $G \otimes_{\mathbb{F}_{q}} \mathbb{F}_{q^{d}}$ is the Iwahori group scheme $I:=\left\{g \in \mathrm{GL}_{d}: g \bmod z\right.$ islowertriangular $\}$. Let $b=\Pi \in L G\left(\mathbb{F}_{q}\right)=$ $D^{\times}$and let the bound $\hat{Z}$ be represented over $R=\mathbb{F}_{q^{d}} \llbracket \zeta \rrbracket$ by

$$
\hat{Z}_{R}:=L^{+} I \cdot T^{-1} \cdot L^{+} I / L^{+} I \subset \widehat{\mathcal{F} \ell_{I, R}} \cong \widehat{\mathcal{F} \ell_{G, R}} .
$$

Its reflex ring is $R_{\hat{Z}}=\mathbb{F}_{q} \llbracket \zeta \rrbracket$ and $\breve{\mathcal{H}}_{G, \hat{Z}}=\mathbb{P}_{\mathbb{F}((\zeta))}^{d-1}$. The quasi-isogeny group $J_{b}$ equals $\mathrm{GL}_{d}$. We are going to describe $\breve{\mathcal{M}}_{\mathbb{\mathbb { G }}_{0}}^{\hat{Z}^{-1}}$.

The category of $L^{+} G$-torsors over a scheme $S \in \mathcal{N} i l p_{\mathbb{F}_{q} \llbracket \zeta \rrbracket}$ is equivalent to the category of $\mathcal{O}_{S} \llbracket z \rrbracket$-modules with $\mathcal{O}_{D^{\text {opp }}} \widehat{\otimes}_{\mathbb{F}_{q}} \mathcal{O}_{S^{-}}$-action, which are Zariski locally on $S$ of the form $\mathcal{O}_{D} \widehat{\otimes}_{\mathbb{F}_{q}} \mathcal{O}_{S}$, where $\mathcal{O}_{D^{\text {opp }}} \widehat{\otimes}_{\mathbb{F}_{q}} \mathcal{O}_{S}$ acts by multiplication on the right. This equivalence sends an $L^{+} G$-torsor $\mathcal{G}$ that is trivialised over an étale covering $S^{\prime} \rightarrow S$ by $\alpha: \mathcal{G}_{S^{\prime}} \stackrel{\sim}{\longrightarrow}\left(L^{+} G\right)_{S^{\prime}}$ with $h:=p_{1}^{*} \alpha \circ p_{2}^{*} \alpha^{-1} \in L^{+} G\left(S^{\prime \prime}\right)=\left(\mathcal{O}_{D} \otimes_{\mathbb{F}_{q} \llbracket z \rrbracket} \Gamma\left(S^{\prime \prime}, \mathcal{O}_{S^{\prime \prime}}\right) \llbracket z \rrbracket\right)^{\times}$, where $p_{i}: S^{\prime \prime}:=S^{\prime} \times{ }_{S} S^{\prime} \rightarrow S^{\prime}$ is the projection onto the $i$ th factor, to the $\mathcal{O}_{S} \llbracket z \rrbracket$-module $M$ obtained by descent from $M^{\prime}:=\mathcal{O}_{D} \widehat{\otimes}_{\mathbb{F}_{q}} \mathcal{O}_{S^{\prime}}$ with the descent datum $p_{2}^{*} M^{\prime} \stackrel{\sim}{\longrightarrow} p_{1}^{*} M^{\prime}$, $m \mapsto h m$. Then $M$ is Zariski locally trivial by Hilbert 90; see [56, Proposition 2.3]. If $S^{\prime} \in \mathcal{N} i l p_{\mathbb{F}_{q^{d}} \llbracket \zeta \rrbracket}$, then $M^{\prime}=\mathcal{O}_{D} \widehat{\otimes}_{\mathbb{F}_{q}} \mathcal{O}_{S^{\prime}}$ decomposes as a direct sum of eigenspaces $M_{i}^{\prime}$ on which $a \in \mathbb{F}_{q^{d}} \subset \mathcal{O}_{D}$ acts as $a^{q^{i}} \in \mathcal{O}_{S^{\prime}}$ for $i \in \mathbb{Z} / d \mathbb{Z}$. Under the isomorphism $\mathcal{O}_{D} \widehat{\otimes}_{\mathbb{F}_{q}} \mathcal{O}_{S^{\prime}} \cong\left\{g \in \mathcal{O}_{S^{\prime}} \llbracket z \rrbracket^{d \times d}: g \bmod z\right.$ islowertriangular $\}$ the $i$ th eigenspace $M_{i}^{\prime}$ is mapped to the $(d-i)$ th column in the matrix space (for $0 \leq i<d)$. Multiplication with $\Pi$ on the right defines morphisms $\Pi: M_{i}^{\prime} \rightarrow M_{i+1}^{\prime}$. If $\underline{\mathcal{G}}$ is a local $G$-shtuka over such an $S^{\prime}$, then $\tau_{\mathcal{G}}$ maps $\sigma^{*} M_{i}^{\prime}$ to $M_{i+1}^{\prime}\left[\frac{1}{z}\right]$. It is bounded by $\hat{Z}^{-1}$ if and only if for all $i$ the map $\tau_{\mathcal{G}}$ is a morphism $\sigma^{*} M_{i}^{\prime} \rightarrow M_{i+1}^{\prime}$ with cokernel locally free of rank 1 over $S^{\prime}$. This means that 
$M$ is the local shtuka (called 'module de coordonnées' in [39], [40]) of a special formal $\mathcal{O}_{D}$-module of dimension $d$ and height $d^{2}$ in the sense of Drinfeld [35].

The formal scheme $\breve{\mathcal{M}}_{\mathbb{\mathbb { G }}_{0}}^{\hat{Z}^{-1}}=\coprod_{\mathbb{Z}} \widehat{\Omega}^{d}$ and the space $\left(\breve{\mathcal{M}}_{\mathbb{\mathbb { Z }}_{0}}^{\hat{Z}^{-1}}\right)^{\text {an }}=\coprod_{\mathbb{Z}} \Omega^{d}$ are the disjoint unions indexed by the height of the quasi-isogeny $\bar{\delta}$, where

$$
\Omega^{d}:=\mathbb{P}_{\mathbb{F}((\zeta))}^{d-1} \backslash \text { all } \mathbb{F}_{q}((\zeta)) \text {-rational hyperplanes }
$$

is Drinfeld's upper halfspace over $\breve{E}=\mathbb{F}((\zeta))$ and $\widehat{\Omega}^{d}$ is its formal model over $\mathbb{F} \llbracket \zeta \rrbracket$ constructed by Drinfeld, Deligne and Mumford. The representability and structure of $\breve{\mathcal{M}}_{\underline{\underline{G}}_{0}}^{\hat{Z}^{-1}}$ is described in detail in [39, Chapitre II]. The period space $\breve{\mathcal{H}}_{G, \hat{Z}, b}^{n a}=\breve{\mathcal{H}}_{G, \hat{Z}, b}^{w a}$ also equals $\Omega^{d}$ and on each connected component of $\left(\breve{\mathcal{M}}_{\mathbb{G}_{0}}^{\hat{Z}^{-1}}\right)$ an the period morphism is the identity of $\Omega^{d}$. The fibres of $\breve{\pi}$ are in bijection with $\mathbb{Z}=D^{\times} / \mathcal{O}_{D}^{\times}$; compare Proposition 7.16 and Theorem 8.1(a). Note that this example has a $\mathbb{Q}_{p}$-analogue also going back to Drinfeld, which is discussed by [80, 1.44-1.46, 3.54-3.77 and 5.48-5.49]. Our exposition differs from [80] because they take covariant Dieudonné modules of formal $\mathcal{O}_{D}$-modules, whereas the local shtuka functor $[40, \S 2.1]$ is contravariant.

Example 6.15 (The Gross-Hopkins period morphism.). This example is also discussed in [40]. Gross and Hopkins [60], [61] take $G=\mathrm{GL}_{r}$, with the upper triangular Borel subgroup and the diagonal torus. Let $b \in L G\left(\mathbb{F}_{q}\right)$ be the matrix $\bar{T}$ from (6.6), and let the bound $\hat{Z}=\hat{Z}_{\preceq \mu}$ be as in Example 2.7 for $\mu=(0, \ldots, 0,-1) \in \mathbb{Z}^{r} \cong X_{*}(T)$ and with reflex field $E:=E_{\hat{Z}}=\mathbb{F}_{q}((\zeta))$. Then $\hat{Z}^{-1}=\hat{Z}_{\preceq(-\mu)_{\text {dom }}}$ with $(-\mu)_{\text {dom }}=(1,0, \ldots, 0)$; compare Example 2.7. The quasi-isogeny group $J_{b}$ is the unit group of the central skew field over $\mathbb{F}_{q}((z))$ with Hasse invariant $1 / r$. The Rapoport-Zink space is the Lubin-Tate space

$$
\breve{\mathcal{M}}_{\underline{\mathbb{G}}_{0}}^{\hat{Z}^{-1}}=\coprod_{\mathbb{Z}} \operatorname{Spf} \mathbb{F} \llbracket \zeta, u_{1}, \ldots, u_{r-1} \rrbracket
$$

of 1-dimensional formal $\mathbb{F}_{q} \llbracket z \rrbracket$-modules of height $r$. Its connected components are indexed by the height of the quasi-isogeny $\bar{\delta}$; that is, the image of $\bar{\delta} \in \mathcal{F} \ell_{\mathrm{GL}_{r}}$ under the map $\mathcal{F} \ell_{\mathrm{GL}_{r}} \rightarrow \pi_{0}\left(\mathcal{F} \ell_{\mathrm{GL}_{r}}\right)=\pi_{1}\left(\mathrm{GL}_{r}\right)=\mathbb{Z}$. The period space is $\breve{\mathcal{H}}_{G, \hat{Z}, b}^{n a}=\breve{\mathcal{H}}_{G, \hat{Z}}^{\text {an }}=\mathbb{P}_{\mathbb{F}((\zeta))}^{r-1} ;$ compare [50, Example 3.3.1]. To define the Hodge-Pink structure on the universal formal $\mathbb{F}_{q} \llbracket z \rrbracket$ module, Gross and Hopkins [61, §11] used the universal additive extension. See [53, Remark 2.5.43] for a comparison of this definition with our definition of the HodgePink structure in Remark 6.4. In [61, §23] they constructed the period morphism $\breve{\pi}$ and showed that its image is $\left(\mathbb{P}_{\breve{E}}^{r-1}\right)^{\text {an }}$; compare Theorem 8.1(a). Note that Gross and Hopkins treated the $\mathbb{Q}_{p}$-analogue simultaneously; see also [80, 5.50].

Example 6.16 (The $\boldsymbol{\zeta}$-adic Carlitz logarithm.). The following example was computed by Breutmann [24]. Let $G=\mathrm{GL}_{2}$, and let the Borel, the maximal torus, the bound $\hat{Z}=\hat{Z}_{\preceq \mu}$ and $\mu$ be as in the previous example. Let $b=\left(\begin{array}{cc}z & 0 \\ 0 & 1\end{array}\right)$. Then $J_{b}$ is the diagonal torus in $\mathrm{GL}_{2}$. The Rapoport-Zink space descends to $\mathbb{F}_{q} \llbracket \zeta \rrbracket$ as the formal scheme

$$
\mathcal{M}_{\underline{\mathbb{G}}_{0}}^{\hat{Z}^{-1}}=\coprod_{(i, j) \in \mathbb{Z}^{2}} \operatorname{Spf} \mathbb{F}_{q} \llbracket \zeta, h \rrbracket,
$$


whose underlying affine Deligne-Lusztig variety $X_{Z^{-1}}(b)=\coprod_{\mathbb{Z}^{2}} \operatorname{Spec} \mathbb{F}_{q}$ is 0 -dimensional. Over the component $(i, j) \in \mathbb{Z}^{i}$, the universal local $\mathrm{GL}_{2}$-shtuka $\underline{\mathcal{G}}$ is given by the local shtuka $\underline{M}(\underline{\mathcal{G}})=\left(\mathbb{F}_{q} \llbracket \zeta, h \rrbracket \llbracket z \rrbracket^{2}, \tau_{M}\right)$ with $\tau_{M}=\left(\begin{array}{cc}z-\zeta & 0 \\ h & 1\end{array}\right)$; see Remark 6.4. The universal quasiisogeny

$$
\bar{\delta}=\left(\begin{array}{cc}
z^{i} & 0 \\
-z^{j} \sum_{\nu=0}^{\infty} h^{q^{\nu}} / z^{\nu+1} & z^{j}
\end{array}\right)
$$

lifts to

$$
\Delta=\left(\begin{array}{cc}
z^{i} \prod_{\nu=0}^{\infty} \frac{z}{z-\zeta^{q^{\nu}}} & 0 \\
-z^{j} \sum_{\nu=0}^{\infty} \frac{h^{q^{\nu}}}{(z-\zeta) \cdots\left(z-\zeta^{q^{\nu}}\right)} & z^{j}
\end{array}\right)
$$

The $E$-analytic space $\left(\breve{\mathcal{M}}_{\mathbb{\mathbb { G }}_{0}}^{\hat{Z}^{-1}}\right)$ an is the disjoint union indexed by $(i, j) \in \mathbb{Z}^{2}$ of the open unit discs with coordinate $h$ and $\mathcal{H}_{G, \hat{Z}, b}^{n a}=\mathcal{H}_{G, \hat{Z}, b}^{w a}=\mathbb{A}_{E}^{1}=\mathbb{P}_{E}^{1} \backslash\{(0: 1)\} \subset \mathcal{H}_{G, \hat{Z}}^{\text {an }}=\mathbb{P}_{E}^{1}$; compare [50, Example 3.3.3]. On the component $(i, j)$ the period morphism $\breve{\pi}$ is given by $\left.h \mapsto \zeta^{j-i}\left(\sigma^{*} t_{-}\right)\right|_{z=\zeta} \log _{\text {Carlitz }}(h)$, where $t_{-}$was defined in $(4.3)$ and $\log _{\text {Carlitz }}(h):=$ $\sum_{\nu=0}^{\infty} \frac{h^{q^{\nu}}}{\left(\zeta-\zeta^{q}\right) \cdots\left(\zeta-\zeta^{q^{\nu}}\right)}$ is the $\zeta$-adic Carlitz logarithm; see [42, §3.4]. In particular, $\breve{\pi}$ is surjective onto $\mathcal{H}_{G, \hat{Z}, b}^{n a}$; compare Theorem 8.1(a). This example is analogous to the period morphism for $p$-divisible groups $[80,5.51,5.52]$ given by the $p$-adic logarithm, which was constructed by Dwork; compare $[66, \S \S 7,8]$.

\section{The tower of étale coverings}

In this section we fix a local $G$-shtuka $\underline{\mathbb{G}}_{0}$ over $\mathbb{F}$ and a bound $\hat{Z}$ with reflex $\operatorname{ring} R_{\hat{Z}}=$ $\kappa \llbracket \xi \rrbracket$. Let again $E_{\hat{Z}}=\kappa((\xi))$ and $\breve{E}=\mathbb{F}((\xi))$ and $\breve{R}_{\hat{Z}}=\mathbb{F} \llbracket \xi \rrbracket$. We write $\breve{\mathcal{M}}$ for the strictly $\breve{E}$-analytic space $\left(\breve{\mathcal{M}}_{\mathbb{G}_{0}}^{\hat{Z}^{-1}}\right)$ an. We shall construct a tower of finite étale coverings of $\breve{\mathcal{M}}$ obtained by trivialising the Tate module of the universal étale local $G$-shtuka $\underline{\mathcal{G}}$ over $\breve{\mathcal{M}}$ from Remark 6.7.

We start more generally with a field extension $L / \breve{E}$ that is complete with respect to an absolute value extending the absolute value on $\breve{E}$ and with an étale local $G$-shtuka $\underline{\mathcal{G}}$ over a connected strictly $L$-analytic space $X$ as in Definition 6.8. We choose a geometric base point $\bar{x}$ of $X$.

Definition 7.1. Let $\rho: G \rightarrow \mathrm{GL}_{r}$ be in $\operatorname{Rep}_{\mathbb{F}_{q} \llbracket z \rrbracket} G$. Let $\underline{M}=\left(M, \tau_{M}\right)$ be the étale local shtuka of rank $r$ associated with the étale local $\mathrm{GL}_{r}$-shtuka $\rho_{*} \underline{\mathcal{G}}$ obtained from $\underline{\mathcal{G}}$ via $\rho$, see $[4, \S 3]$, and let $\underline{M}_{\bar{x}}$ denote its fibre over $\bar{x}$. Then the (dual) Tate module $\check{T}_{\mathcal{G}, \bar{x}}(\rho)$ of $\underline{\mathcal{G}}$ with respect to $\rho$ is defined as the (dual) Tate module of $\underline{M}_{\bar{x}}$,

$$
\check{T}_{\underline{\mathcal{G}}, \bar{x}}(\rho):=\check{T}_{z} \underline{M}_{\bar{x}}:=\left\{m \in \underline{M}_{\bar{x}}: \tau_{M}\left(\sigma^{*} m\right)=m\right\} .
$$

By [94, Proposition 6.1] it is a free $\mathbb{F}_{q} \llbracket z \rrbracket$-module of rank $r$ with a continuous monodromy action of $\pi_{1}^{\text {ett }}(X, \bar{x})$. This action factors through $\pi_{1}^{\text {alg }}(X, \bar{x})$. 
Let now $\rho: G \rightarrow \mathrm{GL}_{r}$ be in $\operatorname{Rep}_{\mathbb{F}_{q}((z))} G$. Let $\underline{N}=\left(N, \tau_{N}\right)$ be the locally free $\mathcal{O}_{X}((z))$ module of rank $r$ and the $\sigma$-linear isomorphism associated with $\rho_{*} L \underline{\mathcal{G}}$ obtained from $L \underline{\mathcal{G}}$ via $\rho$. Let $\underline{N}_{\bar{x}}$ denote its fibre over $\bar{x}$. The rational (dual) Tate module $\check{V}_{\underline{\mathcal{G}}, \bar{x}}(\rho)$ of $\underline{\mathcal{G}}$ with respect to $\rho$ is

$$
\check{V}_{\underline{\mathcal{G}}, \bar{x}}(\rho):=\left\{n \in \underline{N}_{\bar{x}}: \tau_{N}\left(\sigma^{*} n\right)=n\right\},
$$

a finite-dimensional $\mathbb{F}_{q}((z))$-vector space with a continuous monodromy action of $\pi_{1}^{\text {ét }}(X, \bar{x})$.

Remark 7.2. As was pointed out to us by S. Neupert (see also [76, 2.6]), one can also use the following direct way to define the Tate module of an étale local $G$-shtuka that does not use tensor functors. Let $\underline{\mathcal{G}}=\left(\mathcal{G}, \tau_{\mathcal{G}}\right)$ be an étale local $G$-shtuka over a base scheme or a strictly $\mathbb{F}((\zeta))$-analytic space $S$; in other words, $\tau_{\mathcal{G}}$ induces an isomorphism $\tau_{\mathcal{G}}: \sigma^{*} \mathcal{G} \stackrel{\sim}{\longrightarrow}$. Consider for each $n \in \mathbb{N}$ the $\tau$-invariants of the induced map $\sigma^{*} \mathcal{G}_{n} \rightarrow \mathcal{G}_{n}$. Here $\mathcal{G}_{n}$ is the $G / G_{n}$-torsor induced by $\mathcal{G}$ where $G_{n}$ is the kernel of the projection $G\left(\mathbb{F}_{q}[[z]]\right) \rightarrow$ $G\left(\mathbb{F}_{q}[[z]] /\left(z^{n}\right)\right)$. These $\tau$-invariants form a $G\left(\mathbb{F}_{q}[[z]] /\left(z^{n}\right)\right)$-torsor that is trivialised by a finite étale covering. One can then define the Tate module of $\underline{\mathcal{G}}$ as the inverse limit over $n$ of these torsors.

Remark 7.3. (a) Let $\left(\rho^{\prime}, V\right) \in \operatorname{Rep}_{\mathbb{F}_{q}((z))} G$. Let $\Lambda_{0}$ be any $\mathbb{F}_{q} \llbracket z \rrbracket$-lattice in $V$. Then the stabiliser in $G\left(\mathbb{F}_{q} \llbracket z \rrbracket\right)$ of $\Lambda_{0}$ is open and, in particular, of finite index in the compact group $G\left(\mathbb{F}_{q} \llbracket z \rrbracket\right)$. Therefore,

$$
\Lambda:=\bigcap_{g \in G\left(\mathbb{F}_{q} \llbracket z \rrbracket\right)} \rho^{\prime}(g)\left(\Lambda_{0}\right)
$$

is an intersection of finitely many translations of $\Lambda_{0}$ and hence a lattice in $V$. By definition, $\Lambda$ is $G\left(\mathbb{F}_{q} \llbracket z \rrbracket\right)$-invariant. Thus, $\rho^{\prime}$ is induced by $(\rho, \Lambda):=\left(\left.\rho^{\prime}\right|_{\Lambda}, \Lambda\right) \in$ $\operatorname{Rep}_{\mathbb{F}_{q} \llbracket z \rrbracket} G$. From the definition above, we obtain

$$
\check{V}_{\underline{\mathcal{G}}, \bar{x}}\left(\rho^{\prime}\right)=\check{T}_{\underline{\mathcal{G}}, \bar{x}}(\rho) \otimes_{\mathbb{F}_{q} \llbracket z \rrbracket} \mathbb{F}_{q}((z)) .
$$

In particular, the vector space $\check{V}_{\mathcal{G}, \bar{x}}\left(\rho^{\prime}\right)$ is of $\operatorname{dimension} \operatorname{dim} V$.

(b) These definitions are independent of the chosen base point $\bar{x}$, because for any other geometric base point $\bar{x}^{\prime}$ of $X$ there is an isomorphism of fibre functors $\check{T}_{\mathcal{G}, \bar{x}} \cong \check{T}_{\mathcal{G}}, \bar{x}^{\prime}$ and $\check{V}_{\underline{\mathcal{G}}, \bar{x}} \cong \check{V}_{\mathcal{\mathcal { G }}, \bar{x}^{\prime}}$ by [31, Theorem 2.9] and Remark 7.2.

(c) From the definition one obtains that the Tate module and the rational Tate module are tensor functors

$$
\begin{aligned}
& \check{T}_{\underline{\mathcal{G}}, \bar{x}}: \operatorname{Rep}_{\mathbb{F}_{q} \llbracket z \rrbracket} G \longrightarrow \operatorname{Rep}_{\mathbb{F}_{q} \llbracket z \rrbracket}^{\text {cont }}\left(\pi_{1}^{\text {ét }}(X, \bar{x})\right)=\operatorname{Rep}_{\mathbb{F}_{q} \llbracket z \rrbracket}^{\text {cont }}\left(\pi_{1}^{\text {alg }}(X, \bar{x})\right) \quad \text { and } \\
& \check{V}_{\underline{\mathcal{G}}, \bar{x}}: \operatorname{Rep}_{\mathbb{F}_{q}((z))} G \longrightarrow \operatorname{Rep}_{\mathbb{F}_{q}((z))}^{\operatorname{cont}}\left(\pi_{1}^{\text {ét }}(X, \bar{x})\right)
\end{aligned}
$$

In terms of Definition 5.2, we may view $\check{T}_{\underline{\mathcal{G}}, \bar{x}}$ and $\check{V}_{\underline{\mathcal{G}}, \bar{x}}$ as tensor functors

$$
\begin{gathered}
\check{T}_{\underline{\mathcal{G}}}: \operatorname{Rep}_{\mathbb{F}_{q} \llbracket z \rrbracket} G \longrightarrow \mathbb{F}_{q} \llbracket z \rrbracket-\underline{\operatorname{Loc}}_{X} \quad \text { and } \\
\check{V}_{\underline{\mathcal{G}}}: \operatorname{Rep}_{\mathbb{F}_{q}((z))} G \longrightarrow \mathbb{F}_{q}((z))-\underline{\operatorname{Loc}}_{X},
\end{gathered}
$$


with $\check{T}_{\underline{\mathcal{G}}, \bar{x}}=F_{\bar{x}}^{\text {ét }} \circ \check{T}_{\underline{\mathcal{G}}}$ and $\check{V}_{\underline{\mathcal{G}}, \bar{x}}=F_{\bar{x}}^{\text {ét }} \circ \check{V}_{\underline{\mathcal{G}}}$. The tensor functors (7.1) and (7.2) also exist if $X$ is not connected.

Furthermore, $\check{T}_{\mathcal{G}, \bar{x}}$ and $\check{T}_{\underline{\mathcal{G}}}$ are functorial on the category of étale local $G$-shtukas $\underline{\mathcal{G}}$ with isomorphisms as morphisms, and $\check{V}_{\underline{\mathcal{G}}, \bar{x}}$ and $\check{V}_{\underline{\mathcal{G}}}$ are functorial on the category of étale local $G$-shtukas $\underline{\mathcal{G}}$ with isogenies as morphisms. Indeed, an isomorphism, respectively an isogeny, of étale local $G$-shtukas canonically induces an isomorphism between the corresponding $\underline{M}$, respectively $\underline{N}$.

Recall the forgetful functors $\omega_{A}^{\circ}: \operatorname{Rep}_{A} G \rightarrow \operatorname{FMod}_{A}$ and forget $: \operatorname{Rep}_{A}^{\text {cont }}\left(\pi_{1}^{\text {ét }}(X, \bar{x})\right) \rightarrow$ $\operatorname{FMod}_{A}$ from Definition 5.1. For an étale local $G$-shtuka $\underline{\mathcal{G}}$ over $X$, the sets

$$
\begin{aligned}
\operatorname{Triv}_{\underline{\mathcal{G}}, \bar{x}}\left(\mathbb{F}_{q} \llbracket z \rrbracket\right) & :=\operatorname{Isom}^{\otimes}\left(\omega_{\mathbb{F}_{q} \llbracket z \rrbracket}^{\circ}, \text { forget } \circ \check{T}_{\underline{\mathcal{G}}, \bar{x}}\right)\left(\mathbb{F}_{q} \llbracket z \rrbracket\right) \quad \text { and } \\
\operatorname{Triv}_{\underline{\mathcal{G}}, \bar{x}}\left(\mathbb{F}_{q}((z))\right) & :=\operatorname{Isom}^{\otimes}\left(\omega^{\circ}, \text { forget } \circ \check{V}_{\underline{\mathcal{G}}, \bar{x}}\right)\left(\mathbb{F}_{q}((z))\right)
\end{aligned}
$$

are nonempty; see [4, after Definition 3.5]. This is due to the fact that we assumed $G$ to have connected fibres. In [80, 5.32], where nonconnected orthogonal groups are also allowed, the isomorphism class of the étale fibre functor analogous to forget $\circ \check{T}_{\underline{\mathcal{G}}, \bar{x}}$ can vary. By the definition of the Tate functor, $\operatorname{Triv}_{\underline{\mathcal{G}}, \bar{x}}\left(\mathbb{F}_{q} \llbracket z \rrbracket\right)$ carries an action of $G\left(\mathbb{F}_{q} \llbracket z \rrbracket\right) \times \pi_{1}^{\text {alg }}(X, \bar{x})$ where the first factor acts through $\omega_{\mathbb{F}_{q} \llbracket z \rrbracket}^{\circ}$ and the action of $\pi_{1}^{\text {alg }}(X, \bar{x})$ is induced by the action on the Tate functor. Similarly, $\operatorname{Triv}_{\underline{\mathcal{G}}, \bar{x}}\left(\mathbb{F}_{q}((z))\right)$ admits an action of $G\left(\mathbb{F}_{q}((z))\right) \times \pi_{1}^{\text {ét }}(X, \bar{x})$. For every choice of an element $\eta \in \operatorname{Triv}_{\underline{\mathcal{G}}, \bar{x}}\left(\mathbb{F}_{q} \llbracket z \rrbracket\right)$, we obtain a $G\left(\mathbb{F}_{q} \llbracket z \rrbracket\right)$-equivariant bijection

$$
G\left(\mathbb{F}_{q} \llbracket z \rrbracket\right) \stackrel{\sim}{\longrightarrow} \operatorname{Triv}_{\underline{\mathcal{G}}, \bar{x}}\left(\mathbb{F}_{q} \llbracket z \rrbracket\right), \quad g \longmapsto \eta \circ g,
$$

where $G\left(\mathbb{F}_{q} \llbracket z \rrbracket\right)$ acts on itself by multiplication on the right. Under this bijection, the action of $\pi_{1}^{\text {alg }}(X, \bar{x})$ corresponds to a group homomorphism

$$
\pi_{1}^{\mathrm{alg}}(X, \bar{x}) \longrightarrow G\left(\mathbb{F}_{q} \llbracket z \rrbracket\right), \quad h \longmapsto \eta^{-1} \circ h(\eta)
$$

that is independent of $\eta$ up to conjugation by elements of $G\left(\mathbb{F}_{q} \llbracket z \rrbracket\right)$. Similar statements hold for $\operatorname{Triv}_{\mathcal{G}, \bar{x}}\left(\mathbb{F}_{q}((z))\right)$ with $G\left(\mathbb{F}_{q} \llbracket z \rrbracket\right)$ and $\pi_{1}^{\text {alg }}(X, \bar{x})$ replaced by $G\left(\mathbb{F}_{q}((z))\right)$ and $\pi_{1}^{\text {ét }}(X, \bar{x})$.

Definition 7.4. Let $\underline{\mathcal{G}}$ be an étale local $G$-shtuka over a connected $\mathbb{F}((\zeta))$-analytic space $X$, and let $K \subset G\left(\mathbb{F}_{q} \llbracket z \rrbracket\right)$ be an open subgroup. Then an integral K-level structure on $\underline{\mathcal{G}}$ is a $\pi_{1}^{\text {alg }}(X, \bar{x})$-invariant $K$-orbit in $\operatorname{Triv}_{\underline{\mathcal{G}}, \bar{x}}\left(\mathbb{F}_{q} \llbracket z \rrbracket\right)$.

If $K \subset G\left(\mathbb{F}_{q}((z))\right)$ is an open compact subgroup, a rational K-level structure on $\underline{\mathcal{G}}$ is a $\pi_{1}^{\text {ét }}(X, \bar{x})$-invariant $K$-orbit in $\operatorname{Triv}_{\underline{\mathcal{G}}, \bar{x}}\left(\mathbb{F}_{q}((z))\right)$. For nonconnected $X$ we make a similar definition choosing a base point on each connected component and an integral, respectively rational $K$-level structure on the restriction to each connected component separately. Note that every integral $K$-level structure on $\underline{\mathcal{G}}$ defines a rational $K$-level structure but not conversely.

For an open subgroup $K \subset G\left(\mathbb{F}_{q} \llbracket z \rrbracket\right)$, let $X^{K}$ be the functor on the category of $L$-analytic spaces over $X$ parametrising integral $K$-level structures on the étale local $G$-shtuka $\underline{\mathcal{G}}$ over $X$. 
Proposition 7.5. (a) $X^{K}$ is represented by the finite étale covering space of $X$ that corresponds to the finite $\pi_{1}^{\text {alg }}(X, \bar{x})$-set $\operatorname{Triv}_{\underline{\mathcal{G}}, \bar{x}}\left(\mathbb{F}_{q} \llbracket z \rrbracket\right) / K$ under the equivalence (5.4). In particular $X^{K}$ is a strictly L-analytic space.

(b) For $K_{0}=G\left(\mathbb{F}_{q} \llbracket z \rrbracket\right)$, the morphism assigning to $\mathcal{G}$ the $K_{0}$-orbit of all elements of $\operatorname{Triv}_{\underline{\mathcal{G}}, \bar{x}}\left(\mathbb{F}_{q} \llbracket z \rrbracket\right)$ induces an isomorphism $X \cong X^{K_{0}}$.

(c) For any inclusion of open subgroups $\left.K^{\prime} \subset K \subset G\left(\mathbb{F}_{q} \llbracket z \rrbracket\right)\right)$, forgetting part of the level structure induces compatible finite étale surjective morphisms

$$
\breve{\pi}_{K, K^{\prime}}: X^{K^{\prime}} \rightarrow X^{K}
$$

which are Galois with Galois group $K / K^{\prime}$ if $K^{\prime}$ is normal in $K$.

Proof. Denote by $\widetilde{X}^{K}$ the finite étale covering space of $X$ from (a). Let $f: Y \rightarrow X$ be a connected $L$-analytic space over $X$ and let $\eta K$ be an integral $K$-level structure on $f^{*} \underline{\mathcal{G}}$; that is, $\eta \in \operatorname{Triv}_{f^{*} \underline{\mathcal{G}}, \bar{y}}\left(\mathbb{F}_{q} \llbracket z \rrbracket\right)$ and the $K$-orbit $\eta K$ is $\pi_{1}^{\text {alg }}(Y, \bar{y})$-invariant where $\bar{y}$ is a geometric base point of $Y$. We must show that $\eta K$ arises from a uniquely determined $X$-morphism $Y \rightarrow \widetilde{X}^{K}$. Moving $\bar{x}$ by Remark $7.3(\mathrm{~b})$, we may assume that $f(\bar{y})=\bar{x}$, and hence $\check{T}_{f * \mathcal{G}, \bar{y}}=\check{T}_{\mathcal{G}, \bar{x}}$. Consider the finite étale covering space $\widetilde{X}^{K} \times_{X} Y \rightarrow Y$. Then $F_{Y, \bar{y}}^{\text {ét }}\left(\widetilde{X}^{K} \times_{X} Y\right)=F_{X, \bar{x}}^{\text {ét }}\left(\widetilde{X}^{K}\right)=\operatorname{Triv}_{\mathcal{G}}, \bar{x}\left(\mathbb{F}_{q} \llbracket z \rrbracket\right) / K$ for the étale fibre functors from (5.3). In particular, the element $\eta K$ defines a $\pi_{1}^{\text {alg }}(Y, \bar{y})$-equivariant map from the one-element set $\{\bar{y}\}=F_{Y, \bar{y}}^{\text {ét }}(Y)$ to $F_{Y, \bar{y}}^{\text {ét }}\left(\widetilde{X}^{K} \times_{X} Y\right)$. By [31, Theorem 2.10], this map corresponds to a uniquely determined $Y$-morphism $Y \rightarrow \widetilde{X}^{K} \times_{X} Y$. The projection $Y \rightarrow \widetilde{X}^{K}$ onto the first component is the desired $X$-morphism that induces the integral $K$-level structure $\eta K$ over $Y$.

(b) and (c) follow directly from (a).

For arbitrary $X$ and $\underline{\mathcal{G}}$, Proposition 7.5 is the best one can hope for. However, if $X=\left(\breve{\mathcal{M}}_{\mathbb{G}_{0}}^{\hat{Z}^{-1}}\right)^{\text {an }}$, one can even replace $\check{T}_{\underline{\mathcal{G}}, \bar{x}}$ and $\operatorname{Triv}_{\underline{\mathcal{G}}, \bar{x}}\left(\mathbb{F}_{q} \llbracket z \rrbracket\right)$ by $\check{V}_{\underline{\mathcal{G}}, \bar{x}}$ and $\operatorname{Triv}_{\underline{\mathcal{G}}, \bar{x}}\left(\mathbb{F}_{q}((z))\right)$ and allow compact open subgroups $K \subset G\left(\mathbb{F}_{q}((z))\right)$; see Corollaries 7.11 and 7.13. To explain this (also as a preparation to define rational level structures in Definition 7.10), we keep the field $L$ introduced at the beginning of this section and consider in the following an admissible $\mathcal{O}_{L}$-algebra $B$ in the sense of Raynaud; that is, $B$ is a quotient $\beta: \mathcal{O}_{L}\left\langle X_{1}, \ldots, X_{s}\right\rangle \rightarrow B$ that is $\zeta$-torsion free; see (6.3) and [18, p. 293]. Then $\mathcal{X}:=\operatorname{Spf} B$ is an admissible formal $\mathcal{O}_{L}$-scheme. Let $B\left[\frac{1}{\zeta}\right]$ be the associated strictly affinoid $L$-algebra. We equip $B\left[\frac{1}{\zeta}\right]$ with the quotient map $\beta: L\left\langle X_{1}, \ldots, X_{s}\right\rangle \rightarrow B\left[\frac{1}{\zeta}\right]$ and the $L$-Banach norm $|b|:=\inf \left\{|f|_{\text {sup }}: f \in \beta^{-1}(b)\right\}$, where $|f|_{\text {sup }}$ denotes the Gauß norm on the Tate algebra $L\left\langle X_{1}, \ldots, X_{s}\right\rangle$. Then $B=\left\{b \in B\left[\frac{1}{\zeta}\right]:|b| \leq 1\right\}$. The Berkovich spectrum $X=\operatorname{BSpec} B\left[\frac{1}{\zeta}\right]$ is the $L$-analytic space $\mathcal{X}^{\text {an }}$ associated with the formal scheme $\mathcal{X}$.

Lemma 7.6. Recall the notation from (6.1). Let $\left.f=\sum_{i \in \mathbb{Z}} b_{i} z^{i} \in B \llbracket z, z^{-1}\right\}$ and $a \in B$ with $|a|<1$ and assume that $f \in B \llbracket z \rrbracket$ or $a \in \mathcal{O}_{L} \backslash\{0\}$. If $f(a)=\sum_{i \in \mathbb{Z}} b_{i} a^{i}=0$ in $B\left[\frac{1}{\zeta}\right]$, then $f=$ $(z-a) \cdot g$ for a uniquely determined $\left.g=\sum_{n \in \mathbb{Z}} c_{n} z^{n} \in B \llbracket z, z^{-1}\right\}$ with $c_{n}=\sum_{i>n} b_{i} a^{i-n-1} \in B$. Moreover, if $f \in B \llbracket z \rrbracket$ then also $g \in B \llbracket z \rrbracket$. 
Proof. First of all, $b_{i} \in B$ and $|a|<1$ implies that the series $c_{n}:=\sum_{i>n} b_{i} a^{i-n-1}$ converge in $B$ for all $n \in \mathbb{Z}$. One easily computes that $f=(z-a) \cdot g$ for $g:=\sum_{n \in \mathbb{Z}} c_{n} z^{n}$. To prove uniqueness, let $\left.\tilde{g}=\sum_{n \in \mathbb{Z}} \tilde{c}_{n} z^{n} \in B \llbracket z, z^{-1}\right\}$ also satisfy $f=(z-a) \cdot \tilde{g}$. Setting $c_{n}^{\prime}:=c_{n}-\tilde{c}_{n}$ yields $c_{n-1}^{\prime}=a c_{n}^{\prime}$, whence $c_{n}^{\prime}=a^{m-n} c_{m}^{\prime}$ for all $m \geq n$. Letting $m$ go to $\infty$ and using $c_{m}^{\prime} \in B$ shows that $\left|c_{n}^{\prime}\right|$ is arbitrarily small, and therefore $c_{n}^{\prime}=0$ for all $n$. This proves the uniqueness of $g$.

If $f \in B \llbracket z \rrbracket$ and $n<0$, then $c_{n}=a^{-n-1} \cdot f(a)=0$ and therefore $g \in B \llbracket z \rrbracket$. If $a \in \mathcal{O}_{L} \backslash\{0\}$, we must verify the convergence condition $\lim _{n \rightarrow-\infty}\left|c_{n}\right||a|^{r n}=0$ for all $r \geq 1$. We compute $c_{n}=-\sum_{i \leq n} b_{i} a^{i-n-1}$. If $i \leq n$, then $|a|^{(r-1) n} \leq|a|^{(r-1) i}$, and hence

$$
\left|c_{n}\right||a|^{r n} \leq \max _{i \leq n}\left|b_{i}\right||a|^{i-n-1+(r-1) i+n}=\max _{i \leq n}\left|b_{i}\right||a|^{r i-1} .
$$

The latter goes to zero for $n \rightarrow-\infty$ because $\left.f \in B \llbracket z, z^{-1}\right\}$. Therefore, $\left.g \in B \llbracket z, z^{-1}\right\}$.

Remark 7.7. In addition to the loop group $L G$, we consider over $\mathbb{F}_{q} \llbracket \zeta \rrbracket$ the loop groups defined as the fppf-sheaves on $\mathbb{F}_{q} \llbracket \zeta \rrbracket$-algebras $R$ by

$$
L_{z-\zeta} G(R):=G\left(R \llbracket z \rrbracket\left[\frac{1}{z-\zeta}\right]\right) \quad \text { and } \quad L_{z(z-\zeta)} G(R):=G\left(R \llbracket z \rrbracket\left[\frac{1}{z(z-\zeta)}\right]\right)
$$

and the canonical maps of groups $L^{+} G \rightarrow L G \rightarrow L_{z(z-\zeta)} G$ and $L^{+} G \rightarrow L_{z-\zeta} G \rightarrow L_{z(z-\zeta)} G$ that coincide as homomorphisms $L^{+} G \rightarrow L_{z(z-\zeta)} G$. If $\zeta \in R^{\times}$is a unit, note that $z-\zeta \in$ $R \llbracket z \rrbracket^{\times}$, and hence $L_{z-\zeta} G=L^{+} G$ and $L_{z(z-\zeta)} G=L G$. On the other hand, if $\zeta$ is nilpotent in $R$, then $L_{z-\zeta} G=L_{z(z-\zeta)} G=L G$.

Recall from [4, Definition 4.22] that a local $G$-shtuka over an admissible formal $\mathcal{O}_{L^{-}}$ scheme $\mathcal{X}$ can be viewed as a projective system $\left(\underline{\mathcal{G}}_{m}\right)_{m \in \mathbb{N}}$ of local $G$-shtukas $\underline{\mathcal{G}}_{m}$ over $\mathcal{X}_{m}=\mathrm{V}\left(\zeta^{m}\right) \subset \mathcal{X}$ with $\underline{\mathcal{G}}_{m-1} \cong \underline{\mathcal{G}}_{m} \otimes_{\mathcal{X}_{m}} \mathcal{X}_{m-1}$. On $\mathcal{X}_{m}$ the element $\zeta$ is nilpotent.

Now let $B$ and $B\left[\frac{1}{\zeta}\right]$ be as before. If $(\underline{\mathcal{G}}, \bar{\delta})$ is an $\operatorname{Spf} B$-valued point in $\breve{\mathcal{M}}_{\mathbb{G}_{0}}^{\hat{Z}^{-1}}(\operatorname{Spf} B)$, then the étale covering $\operatorname{Spf} B^{\prime} \rightarrow \operatorname{Spf} B$ from Lemma 6.2 is given by a faithfully flat ring homomorphism $B \rightarrow B^{\prime}$ by [18, Lemma 1.6], and this implies that $\underline{\mathcal{G}}$ comes from an $L^{+} G$-torsor $\mathcal{G}$ over $\operatorname{Spec} B$ together with an isomorphism of the associated $L_{z-\zeta} G$-torsors $\tau_{\mathcal{G}}: L_{z-\zeta} \sigma^{*} \mathcal{G} \stackrel{\sim}{\longrightarrow} L_{z-\zeta} \mathcal{G}$. We view $\left(\mathcal{G}, \tau_{\mathcal{G}}\right)$ as the bounded local $G$-shtuka over Spec $B$ induced from the bounded local $G$-shtuka $\underline{\mathcal{G}}$ over $\operatorname{Spf} B$. A quasi-isogeny $u:\left(\mathcal{G}^{\prime}, \tau_{\mathcal{G}^{\prime}}\right) \rightarrow$ $\left(\mathcal{G}, \tau_{\mathcal{G}}\right)$ between two such bounded local $G$-shtukas $\left(\mathcal{G}^{\prime}, \tau_{\mathcal{G}^{\prime}}\right)$ and $\left(\mathcal{G}, \tau_{\mathcal{G}}\right)$ over $\operatorname{Spec} B$ is an isomorphism of the associated $L G$-torsors $u: L \mathcal{G}^{\prime} \stackrel{\sim}{\longrightarrow} L \mathcal{G}$ that satisfies $u \circ \tau_{\mathcal{G}^{\prime}}=\tau_{\mathcal{G}} \circ \sigma^{*} u$ as isomorphism of the associated $L_{z(z-\zeta)} G$-torsors.

In particular, $\left(\mathcal{G}, \tau_{\mathcal{G}}\right)$ induces an étale local $G$-shtuka on the $L$-analytic space $X=$ $(\operatorname{Spf} B)^{\text {an }}=\operatorname{BSpec}\left(B\left[\frac{1}{\zeta}\right]\right)$.

The following proposition is a weaker analogue of the fact that lifts of $p$-divisible groups and morphisms between them correspond uniquely to lifts of the Hodge-filtrations on the associated crystals (respectively morphisms between them). We do not dispose of the full analogue of this assertion because in our (in general nonminuscule) context Griffiths transversality does not hold; compare the discussion of Genestier and Lafforgue in $[41, \S 11]$. 
Proposition 7.8. Let $\mathcal{X}=\operatorname{Spf} B$ be an admissible formal $\mathcal{O}_{L}$-scheme and let $X:=\mathcal{X}^{\text {an }}$ be its associated L-analytic space. Assume that $X$ is connected and choose a geometric base point $\bar{x}$ of $X$. Let $(\underline{\mathcal{G}}, \bar{\delta})$ and $\left(\underline{\mathcal{G}}^{\prime}, \bar{\delta}^{\prime}\right)$ be (representatives of) points in $\breve{\mathcal{M}}_{\mathbb{G}_{0}}^{\hat{Z}^{-1}}(\mathcal{X})$. Then $\breve{\pi}(\underline{\mathcal{G}}, \bar{\delta})=\breve{\pi}\left(\underline{\mathcal{G}}^{\prime}, \bar{\delta}^{\prime}\right)$ in $\breve{\mathcal{H}}_{G, \hat{Z}}^{\text {an }}(X)$ if and only if the unique lift of the quasi-isogeny $\bar{\delta}^{-1} \circ \bar{\delta}^{\prime}$ by rigidity [4, Proposition 2.11] is a quasi-isogeny $u: \underline{\mathcal{G}}^{\prime} \rightarrow \underline{\mathcal{G}}$ over $\mathrm{Spec} B$ in the sense of Remark 7.7. In this case, $u$ induces an isomorphism of the rational Tate module functors $\check{V}_{u, \bar{x}}: \check{V}_{\mathcal{G}^{\prime}, \bar{x}} \stackrel{\sim}{\longrightarrow} \check{V}_{\mathcal{G}, \bar{x}}$ over $X$ and the following assertions are equivalent:

(a) $u: \underline{\mathcal{G}}^{\prime} \rightarrow \underline{\mathcal{G}}$ is an isomorphism of local G-shtukas, that is $(\underline{\mathcal{G}}, \bar{\delta})=\left(\underline{\mathcal{G}}^{\prime}, \bar{\delta}^{\prime}\right)$ in $\breve{\mathcal{M}}_{\underline{\mathbb{G}}_{0}}^{\hat{Z}^{-1}}(\mathcal{X})$,

(b) $\check{V}_{u, \bar{x}}$ is an isomorphism $\check{T}_{\underline{\mathcal{G}}^{\prime}, \bar{x}} \stackrel{\sim}{\longrightarrow} \check{T}_{\underline{\mathcal{G}}, \bar{x}}$ of the integral Tate module functors,

(c) $\check{V}_{u, \bar{x}}(\rho)$ is an isomorphism $\check{T}_{\underline{\mathcal{G}}^{\prime}, \bar{x}}(\rho) \stackrel{\sim}{\sim} \check{T}_{\mathcal{G}, \bar{x}}(\rho)$ for some faithful $\rho \in \operatorname{Rep}_{\mathbb{F}_{q} \llbracket z \rrbracket} G$.

Moreover, for every rational $G\left(\mathbb{F}_{q} \llbracket z \rrbracket\right)$-level structure $\eta G\left(\mathbb{F}_{q} \llbracket z \rrbracket\right)$ on $\underline{\mathcal{G}}$ with $\eta \in$ $\operatorname{Triv}_{\mathcal{G}, \bar{x}}\left(\mathbb{F}_{q}((z))\right)$ there is an admissible formal blowing-up $\mathcal{Y} \rightarrow \mathcal{X}$ and $\underline{a}\left(\underline{\mathcal{G}}^{\prime \prime}, \bar{\delta}^{\prime \prime}\right) \in$ $\breve{\mathcal{M}}_{\underline{\mathbb{G}}_{0}}^{\hat{Z}^{-1}}(\mathcal{Y})$ with $\breve{\pi}(\underline{\mathcal{G}}, \bar{\delta})=\breve{\pi}\left(\underline{\mathcal{G}}^{\prime \prime}, \bar{\delta}^{\prime \prime}\right)$ and $\left(\check{V}_{u^{\prime \prime}, \bar{x}}\right)^{-1} \circ \eta \in \operatorname{Triv}_{\underline{\mathcal{G}}^{\prime \prime}, \bar{x}}\left(\mathbb{F}_{q} \llbracket z \rrbracket\right)$, where $u^{\prime \prime}: \underline{\mathcal{G}}^{\prime \prime} \rightarrow \underline{\mathcal{G}}$ is the unique lift of $\bar{\delta}^{-1} \circ \bar{\delta}^{\prime \prime}$.

Remark. The last assertion uses the ind-projectivity of the affine flag variety $\mathcal{F} \ell_{G}$ and is in general false if $G$ is not parahoric; see Example 8.6.

Proof of Proposition 7.8. By Lemma 6.2 there is an étale covering $\widetilde{\mathcal{X}}=\operatorname{Spf} \widetilde{B} \rightarrow$ $\mathcal{X}$ of admissible formal $\mathcal{O}_{L^{-}}$-schemes and trivialisations $\alpha: \underline{\mathcal{G}} \stackrel{\sim}{\longrightarrow}\left(\left(L^{+} G\right)_{\tilde{\mathcal{X}}}, A \sigma^{*}\right)$ and $\alpha^{\prime}: \underline{\mathcal{G}}^{\prime} \stackrel{\sim}{\longrightarrow}\left(\left(L^{+} G\right)_{\widetilde{\mathcal{X}}}, A^{\prime} \sigma^{*}\right)$ with $A, A^{\prime} \in G\left(\widetilde{B} \llbracket z \rrbracket\left[\frac{1}{z-\zeta}\right]\right)$. Note that $\widetilde{B} \subset \widetilde{B}\left[\frac{1}{\zeta}\right]$ because $\widetilde{B}$ has no $\zeta$-torsion. In addition, the quasi-isogenies $\bar{\delta}$ and $\bar{\delta}^{\prime}$ correspond under $\alpha$ and $\alpha^{\prime}$ to elements $\bar{\Delta}, \bar{\Delta}^{\prime} \in L G(\widetilde{B} /(\zeta))$, which lift by Lemma 6.1 to uniquely determined elements $\left.\Delta, \Delta^{\prime} \in G\left(\widetilde{B} \llbracket z, z^{-1}\right\}\left[\frac{1}{t_{-}}\right]\right)$with $\Delta A=b \sigma^{*}(\Delta)$ and $\Delta^{\prime} A^{\prime}=b \sigma^{*}\left(\Delta^{\prime}\right)$. In particular, the quasiisogeny $\bar{\delta}^{-1} \circ \bar{\delta}^{\prime}: \underline{\mathcal{G}} \rightarrow \underline{\mathcal{G}}^{\prime}$ over $\operatorname{Spec} B /(\zeta)$ lifts to $\left.U=\Delta^{-1} \Delta^{\prime} \in G\left(\widetilde{B} \llbracket z, z^{-1}\right\}\left[\frac{1}{t_{-}}\right]\right)$with $U A^{\prime}=A \sigma^{*}(U)$. The morphism $\breve{\pi}$ sends $(\underline{\mathcal{G}}, \bar{\delta})$ and $\left(\underline{\mathcal{G}}^{\prime}, \bar{\delta}^{\prime}\right)$ to

$$
\begin{aligned}
\gamma & :=\sigma^{*}(\Delta) A^{-1} \cdot G\left(\widetilde{B}\left[\frac{1}{\zeta}\right] \llbracket z-\zeta \rrbracket\right) \quad \text { and } \\
\gamma^{\prime} & :=\sigma^{*}\left(\Delta^{\prime}\right)\left(A^{\prime}\right)^{-1} \cdot G\left(\widetilde{B}\left[\frac{1}{\zeta}\right] \llbracket z-\zeta \rrbracket\right) \\
& =\sigma^{*}(\Delta) A^{-1} U \cdot G\left(\widetilde{B}\left[\frac{1}{\zeta}\right] \llbracket z-\zeta \rrbracket\right) .
\end{aligned}
$$

If $u$ is a quasi-isogeny over $\operatorname{Spec} B$, then $U \in G\left(\widetilde{B} \llbracket z \rrbracket\left[\frac{1}{z}\right]\right) \subset G\left(\widetilde{B}\left[\frac{1}{\zeta}\right] \llbracket z-\zeta \rrbracket\right)$ and hence $\breve{\pi}(\underline{\mathcal{G}}, \bar{\delta})=\gamma=\gamma^{\prime}=\breve{\pi}\left(\underline{\mathcal{G}}^{\prime}, \bar{\delta}^{\prime}\right)$ in $\breve{\mathcal{H}}_{G, \hat{Z}}^{\text {an }}$.

Conversely, the condition $\breve{\pi}(\underline{\mathcal{G}}, \bar{\delta})=\breve{\pi}\left(\underline{\mathcal{G}}^{\prime}, \bar{\delta}^{\prime}\right)$ yields $U \in G\left(\widetilde{B}\left[\frac{1}{\zeta}\right] \llbracket z-\zeta \rrbracket\right)$. We claim that this implies $\left.U \in G\left(\widetilde{B} \llbracket z, z^{-1}\right\}\left[\frac{1}{\sigma^{*}\left(t_{-}\right)}\right]\right)$. To prove the claim, let $\rho: G \hookrightarrow \mathrm{GL}_{r}$ be a faithful representation. Then the entries of $\rho(U)$ and $\rho(U)^{-1}$ are of the form $t_{-}^{-e} f$ with $e \in \mathbb{N}_{0}$ and $\left.f \in \widetilde{B} \llbracket z, z^{-1}\right\}$. We must show that there is a $\left.g \in \widetilde{B} \llbracket z, z^{-1}\right\}$ with $t_{-}^{-e} f=\sigma^{*}\left(t_{-}\right)^{-e} g$. Recall from (4.3) that $t_{-}^{-e} f=\left(1-\frac{\zeta}{z}\right)^{-e} \sigma^{*}\left(t_{-}\right)^{-e} f$. If $e>0$, then $U \in G\left(\widetilde{B}\left[\frac{1}{\zeta}\right] \llbracket z-\zeta \rrbracket\right)$, respectively $U^{-1} \in G\left(\widetilde{B}\left[\frac{1}{\zeta}\right] \llbracket z-\zeta \rrbracket\right)$, implies that $f(\zeta)=0$ in $\widetilde{B}\left[\frac{1}{\zeta}\right]$. By Lemma 7.6 we find $f=(z-\zeta) g_{1}=$ 
$\left(1-\frac{\zeta}{z}\right) z g_{1}$ with $\left.g_{1} \in \widetilde{B} \llbracket z, z^{-1}\right\}$, and hence $t_{-}^{-e} f=\left(1-\frac{\zeta}{z}\right)^{1-e} \sigma^{*}\left(t_{-}\right)^{-e} z g_{1}$. Continuing in this way for $\rho(U)$ and $\rho(U)^{-1}$, we obtain that $\left.\rho(U) \in \mathrm{GL}_{r}\left(\widetilde{B} \llbracket z, z^{-1}\right\}\left[\frac{1}{\sigma^{*}\left(t_{-}\right)}\right]\right)$. The claim follows.

This shows that $\left.\sigma^{*}(U) \in G\left(\widetilde{B} \llbracket z, z^{-1}\right\}\left[\frac{1}{\sigma^{2 *}\left(t_{-}\right)}\right]\right) \subset G\left(\widetilde{B}\left[\frac{1}{\zeta}\right] \llbracket z-\zeta^{q} \rrbracket\right)$, where we use (6.2). Because $A, A^{\prime} \in G\left(\widetilde{B}\left[\frac{1}{\zeta}\right] \llbracket z-\zeta^{q^{i}} \rrbracket\right)$ for all $i>0$, we obtain

$$
U=A \sigma^{*}(U)\left(A^{\prime}\right)^{-1} \in G\left(\widetilde{B}\left[\frac{1}{\zeta}\right] \llbracket z-\zeta^{q} \rrbracket\right) .
$$

Analogous to the previous paragraph, this implies that $\left.U \in G\left(\widetilde{B} \llbracket z, z^{-1}\right\}\left[\frac{1}{\sigma^{2 *}\left(t_{-}\right)}\right]\right)$and iteratively $\left.U \in G\left(\widetilde{B} \llbracket z, z^{-1}\right\}\left[\frac{1}{\sigma^{i *}\left(t_{-}\right)}\right]\right)$for all $i>0$. It follows that the entries of $\rho(U)$ converge on all of $\{0<|z|<1\}$, whence lie in $\left.\widetilde{B} \llbracket z, z^{-1}\right\}$, and so $\left.U \in G\left(\widetilde{B} \llbracket z, z^{-1}\right\}\right)$.

Now let $\mathfrak{p} \subset \widetilde{B}\left[\frac{1}{\zeta}\right]$ be a minimal prime ideal and let $x \in \widetilde{\mathcal{X}}^{\text {an }}=\operatorname{BSpec}\left(\widetilde{B}\left[\frac{1}{\zeta}\right]\right)$ be a point given by a multiplicative semi-norm $|\cdot|_{x}: \widetilde{B}\left[\frac{1}{\zeta}\right] \rightarrow \mathbb{R}_{\geq 0}$ such that $\left\{b \in \widetilde{B}\left[\frac{1}{\zeta}\right]:|b|_{x}=\right.$ $0\}=\mathfrak{p}$. Note that $|\cdot|_{x}$ exists by $[7$, Corollary 2.1.16], for example as the preimage of the multiplicative Gauß norm on $L\left\langle X_{1}, \ldots, X_{d}\right\rangle$ under a Noether normalisation map $L\left\langle X_{1}, \ldots, X_{d}\right\rangle \hookrightarrow \widetilde{B}\left[\frac{1}{\zeta}\right] / \mathfrak{p}$; see $[17, \S 6.1 .2$, Theorem 1]. Let $\Omega$ be the completion with respect to $|\cdot|_{x}$ of an algebraic closure of $\widetilde{B}\left[\frac{1}{\zeta}\right] / \mathfrak{p}$ and let $\mathcal{O}_{\Omega}$ be the valuation ring of $\Omega$. Then the image of $\widetilde{B}$ in $\Omega$ lies in $\mathcal{O}_{\Omega}$. We denote the image of $U$ in $\left.G\left(\mathcal{O}_{\Omega} \llbracket z, z^{-1}\right\}\right)$ by $U_{\mathfrak{p}}$. By $\left[4\right.$, Lemma 2.8] there are elements $H_{\mathfrak{p}}, H_{\mathfrak{p}}^{\prime} \in G(\Omega \llbracket z \rrbracket)$ with $A \sigma^{*}\left(H_{\mathfrak{p}}\right)=H_{\mathfrak{p}}$ and $A^{\prime} \sigma^{*}\left(H_{\mathfrak{p}}^{\prime}\right)=H_{\mathfrak{p}}^{\prime}$ that provide a trivialisation of the Tate module functors. By [54, Remark 3.4.3], we even have $\rho\left(H_{\mathfrak{p}}\right), \rho\left(H_{\mathfrak{p}}^{\prime}\right) \in \mathrm{GL}_{r}\left(\Omega\left\langle\frac{z}{\zeta^{s}}\right\rangle\right)$ for every $s>\frac{1}{q}$. We compute $\rho\left(H_{\mathfrak{p}}^{-1} U_{\mathfrak{p}} H_{\mathfrak{p}}^{\prime}\right)=\rho\left(\sigma^{*}\left(H_{\mathfrak{p}}^{-1}\right) A^{-1} U_{\mathfrak{p}} A^{\prime} \sigma^{*}\left(H_{\mathfrak{p}}^{\prime}\right)\right)=\sigma^{*} \rho\left(H_{\mathfrak{p}}^{-1} U_{\mathfrak{p}} H_{\mathfrak{p}}^{\prime}\right) \in \mathrm{GL}_{r}\left(\Omega\left\langle\frac{z}{\zeta^{s}}, z^{-1}\right\}\right)$, and this implies $\rho\left(H_{\mathfrak{p}}^{-1} U_{\mathfrak{p}} H_{\mathfrak{p}}^{\prime}\right) \in \mathrm{GL}_{r}\left(\mathbb{F}_{q}((z))\right)$ because $\Omega\left\langle\frac{z}{\zeta^{s}}, z^{-1}\right\}^{\sigma}=\mathbb{F}_{q}((z))$. Let $N_{\mathfrak{p}} \in \mathbb{N}_{0}$ be minimal such that $z^{N_{\mathfrak{p}}} \rho\left(H_{\mathfrak{p}}^{-1} U_{\mathfrak{p}} H_{\mathfrak{p}}^{\prime}\right), z^{N_{\mathfrak{p}}} \rho\left(H_{\mathfrak{p}}^{-1} U_{\mathfrak{p}} H_{\mathfrak{p}}^{\prime}\right)^{-1} \in \mathbb{F}_{q} \llbracket z \rrbracket^{r \times r}$. Then $N_{\mathfrak{p}}=0$ if and only if $\rho\left(H_{\mathfrak{p}}^{-1} U_{\mathfrak{p}} H_{\mathfrak{p}}^{\prime}\right) \in \mathrm{GL}_{r}\left(\mathbb{F}_{q} \llbracket z \rrbracket\right)$; that is, $H_{\mathfrak{p}}^{-1} U_{\mathfrak{p}} H_{\mathfrak{p}}^{\prime} \in G\left(\mathbb{F}_{q} \llbracket z \rrbracket\right)$. Moreover, $\left.z^{N_{\mathfrak{p}}} \rho\left(U_{\mathfrak{p}}\right), z^{N_{\mathfrak{p}}} \rho\left(U_{\mathfrak{p}}\right)^{-1} \in \Omega\left\langle\frac{z}{\zeta^{s}}\right\rangle^{r \times r} \cap \mathcal{O}_{\Omega} \llbracket z, z^{-1}\right\}^{r \times r}=\mathcal{O}_{\Omega} \llbracket z \rrbracket^{r \times r}$. Because this holds for all of the finitely many minimal prime ideals of $\widetilde{B}\left[\frac{1}{\zeta}\right]$ and the intersection of these is the nil-radical $\mathcal{N}$ of $\widetilde{B}\left[\frac{1}{\zeta}\right]$, we see that $\left.U \in G\left(\widetilde{B} \llbracket z, z^{-1}\right\}\right)$ implies $z^{N} \rho(U), z^{N} \rho\left(U^{-1}\right) \in$ $\widetilde{B} \llbracket z \rrbracket^{r \times r}+\mathcal{N} \llbracket z^{-1} \rrbracket^{r \times r}$ for $N:=\max \left\{N_{\mathfrak{p}}: \mathfrak{p}\right.$ minimal $\}$. Because $\widetilde{B}\left[\frac{1}{\zeta}\right]$ is Noetherian, the nil-radical is nilpotent and there is an integer $m$ such that $\mathcal{N}^{q^{m}}=(0)$. In particular, $z^{N} \rho\left(\sigma^{m *}(U)\right) \in \widetilde{B} \llbracket z \rrbracket^{r \times r}$. Let $n$ be such that $(z-\zeta)^{n} \rho(A),(z-\zeta)^{n} \rho\left(A^{\prime}\right)^{-1} \in \widetilde{B} \llbracket z \rrbracket^{r \times r}$. Then

$$
\begin{aligned}
\left(\prod_{i=0}^{m-1}\left(z-\zeta^{q^{i}}\right)^{2 n}\right) \cdot z^{N} \rho(U)= & (z-\zeta)^{n} \rho(A) \cdot \ldots \cdot \sigma^{(m-1) *}\left((z-\zeta)^{n} \rho(A)\right) \cdot z^{N} \rho\left(\sigma^{m *}(U)\right) \\
& \cdot \sigma^{(m-1) *}\left((z-\zeta)^{n} \rho\left(A^{\prime}\right)^{-1}\right) \cdot \ldots \cdot(z-\zeta)^{n} \rho\left(A^{\prime}\right)^{-1} \\
& \in \widetilde{B} \llbracket z \rrbracket^{r \times r}
\end{aligned}
$$

and applying Lemma 7.6 with $a=\zeta^{q^{i}}$ for $i=0, \ldots, m-1$ to the entries of this matrix yields $z^{N} \rho(U) \in \widetilde{B} \llbracket z \rrbracket^{r \times r}$. In the same way, we see that $z^{N} \rho\left(U^{-1}\right) \in \widetilde{B} \llbracket z \rrbracket^{r \times r}$. This implies $\rho(U) \in \mathrm{GL}_{r}\left(\widetilde{B} \llbracket z \rrbracket\left[\frac{1}{z}\right]\right)$ and $U \in G\left(\widetilde{B} \llbracket z \rrbracket\left[\frac{1}{z}\right]\right)=L G(\widetilde{B})$. We conclude that $U$ defines a quasi- 
isogeny $U: \underline{\mathcal{G}}_{\widetilde{B}} \rightarrow \underline{\mathcal{G}}_{\widetilde{B}}^{\prime}$ which induces the isomorphism of the rational Tate module functors $\check{V}_{U, \bar{x}}=H_{\mathfrak{p}}^{-1} U_{\mathfrak{p}} H_{\mathfrak{p}}^{\prime}: \check{V}_{\underline{\mathcal{G}}^{\prime}, \bar{x}} \stackrel{\sim}{\longrightarrow} \check{V}_{\mathcal{G}, \bar{x}}$ for the geometric base point $\bar{x}: \operatorname{BSpec}(\Omega) \rightarrow X$. By uniqueness $U$ descends to a quasi-isogeny $u: \underline{\mathcal{G}}^{\prime} \rightarrow \underline{\mathcal{G}}$ over $\operatorname{Spec} B$ as desired.

In this situation, clearly (a) implies (b) and (b) implies (c). We further see that (c) for our representation $\rho$ implies $\rho\left(H_{\mathfrak{p}}^{-1} U_{\mathfrak{p}} H_{\mathfrak{p}}^{\prime}\right) \in \mathrm{GL}_{r}\left(\mathbb{F}_{q} \llbracket z \rrbracket\right)$ for all minimal $\mathfrak{p} \subset \widetilde{B}\left[\frac{1}{\zeta}\right]$, and hence the integer $N$ defined above is zero and $U \in G(\widetilde{B} \llbracket z \rrbracket)$. In particular, (c) implies (a), because $u: \underline{\mathcal{G}}^{\prime} \stackrel{\sim}{\longrightarrow}$ is an isomorphism of local $G$-shtukas if and only if $U \in G(\widetilde{B} \llbracket z \rrbracket)$.

It remains to prove the last assertion about the rational $G\left(\mathbb{F}_{q} \llbracket z \rrbracket\right)$-level structure $\eta G\left(\mathbb{F}_{q} \llbracket z \rrbracket\right)$ on $\underline{\mathcal{G}}$ where $\eta \in \operatorname{Triv}_{\underline{\mathcal{G}}, \bar{x}}\left(\mathbb{F}_{q}((z))\right)$. Let $\rho^{\prime \prime}: \pi_{1}^{\text {et }}(X, \bar{x}) \rightarrow G\left(\mathbb{F}_{q} \llbracket z \rrbracket\right)$ be the homomorphism by which the fundamental group acts on $\eta$; that is, $g(\eta)=\eta \cdot \rho^{\prime \prime}(g)$ for $g \in \pi_{1}^{\text {ét }}(X, \bar{x})$. Note that $\rho^{\prime \prime}(g)$ indeed lies in $G\left(\mathbb{F}_{q} \llbracket z \rrbracket\right)$ because $g$ fixes $\eta G\left(\mathbb{F}_{q} \llbracket z \rrbracket\right)$. In particular, $\rho^{\prime \prime}$ factors through a representation $\pi_{1}^{\text {alg }}(X, \bar{x}) \rightarrow G\left(\mathbb{F}_{q} \llbracket z \rrbracket\right)$. By Corollary 5.4, Proposition 5.3 and [4, Proposition 3.6], $\rho^{\prime \prime}$ comes from an étale local $G$-shtuka $\underline{\mathcal{G}}_{L}^{\prime \prime}$ over $\operatorname{Spec} B\left[\frac{1}{\zeta}\right]$ together with a tensor isomorphism $\beta \in \operatorname{Triv}_{\underline{\mathcal{G}}^{\prime \prime}, \bar{x}}\left(\mathbb{F}_{q} \llbracket z \rrbracket\right)$, and the tensor isomorphism $\eta \beta^{-1}: \check{V}_{\underline{\mathcal{G}}_{L}^{\prime \prime}, \bar{x}} \stackrel{\sim}{\longrightarrow} \check{V}_{\underline{\mathcal{G}}, \bar{x}}$ is of the form $\check{V}_{u_{L}^{\prime \prime}, \bar{x}}$ for a quasi-isogeny $u_{L}^{\prime \prime}: \underline{\mathcal{G}}_{L}^{\prime \prime} \rightarrow \underline{\mathcal{G}}_{B\left[\frac{1}{\zeta}\right]}$ over Spec $B\left[\frac{1}{\zeta}\right]$. This means that $\underline{\mathcal{G}}_{L}^{\prime \prime}=\left(\mathcal{G}_{L}^{\prime \prime}, \tau^{\prime \prime}\right)$, where $\mathcal{G}_{L}^{\prime \prime}$ is an $L^{+} G$-torsor over $\operatorname{Spec} B\left[\frac{1}{\zeta}\right]$ and $\tau^{\prime \prime}: \sigma^{*} \mathcal{G}_{L}^{\prime \prime} \stackrel{\sim}{\longrightarrow} \mathcal{G}_{L}^{\prime \prime}$ is an isomorphism of $L^{+} G$-torsors. Also, $u_{L}^{\prime \prime}: L \mathcal{G}_{L}^{\prime \prime} \stackrel{\sim}{\longrightarrow} L \mathcal{G}_{B\left[\frac{1}{\zeta}\right]}$ is an isomorphism of the associated $L G$-torsors with $u_{L}^{\prime \prime} \circ \tau^{\prime \prime}=\tau_{\mathcal{G}} \circ \sigma^{*} u_{L}^{\prime \prime}$. Note that the assumption on the nilpotence of $\zeta$ in [4, Proposition 3.6] is not satisfied for $\operatorname{Spec} B\left[\frac{1}{\zeta}\right]$, but is also not used in the proof of [4, Proposition 3.6]

We may thus apply the following Lemma 7.9 by taking the $L G$-torsor associated with $\mathcal{G}$ as the $L G$-torsor $\mathcal{G}$ in Lemma 7.9. It provides an extension of the pair $\left(\underline{\mathcal{G}}_{L}^{\prime \prime}, u_{L}^{\prime \prime}\right)$ to a local $G$-shtuka $\underline{\mathcal{G}}^{\prime \prime}$ bounded by $\hat{Z}^{-1}$ and a quasi-isogeny $u^{\prime \prime}: \underline{\mathcal{G}}^{\prime \prime} \rightarrow \underline{\mathcal{G}}$ over a blowing-up $Y$ of $\operatorname{Spec} B$ in a finitely generated ideal $\mathfrak{b} \subset B$ containing a power of $\zeta$. By [18, Propositions 2.1 and 1.3] the $\zeta$-adic completion $\mathcal{Y}$ of $Y$ is the admissible formal blowing-up of $\mathcal{X}=\operatorname{Spf} B$ in the ideal $\mathfrak{b}$. In particular, $\mathcal{Y}^{\text {an }} \rightarrow X$ is an isomorphism. We set $\bar{\delta}^{\prime \prime}:=\bar{\delta} \circ\left(u^{\prime \prime} \bmod \zeta\right)$. Then $\left(\underline{\mathcal{G}}^{\prime \prime}, \bar{\delta}^{\prime \prime}\right) \in \breve{\mathcal{M}}_{\underline{\mathbb{G}}_{0}}^{\hat{Z}^{-1}}(\mathcal{Y})$, and $\breve{\pi}(\underline{\mathcal{G}}, \bar{\delta})=\breve{\pi}\left(\underline{\mathcal{G}}^{\prime \prime}, \bar{\delta}^{\prime \prime}\right)$ by the first part of the proposition, and $\left(\check{V}_{u^{\prime \prime}, \bar{x}}\right)^{-1} \circ \eta=\beta \in \operatorname{Triv}_{\mathcal{G}^{\prime \prime}, \bar{x}}\left(\mathbb{F}_{q} \llbracket z \rrbracket\right)$ by construction.

Lemma 7.9. Let $B$ be an admissible formal $\mathcal{O}_{L}$-algebra. Let $\mathcal{G}$ be an LG-torsor over $\operatorname{Spec} B$ and assume that there is an étale covering $\operatorname{Spf} \widetilde{B} \rightarrow \operatorname{Spf} B$ of admissible formal $\mathcal{O}_{L}$-schemes such that $\mathcal{G}$ admits a trivialisation $\alpha: \mathcal{G} \otimes_{B} \operatorname{Spec} \widetilde{B} \stackrel{\sim}{\longrightarrow} L G_{\text {Spec } \widetilde{B}}$. Let $\tau_{\mathcal{G}}: L_{z(z-\zeta)} \sigma^{*} \mathcal{G} \stackrel{\sim}{\longrightarrow} L_{z(z-\zeta)} \mathcal{G}$ be an isomorphism of the associated $L_{z(z-\zeta)} G$-torsors. Furthermore, let $\mathcal{G}_{L}^{\prime \prime}$ be an $L^{+} G$-torsor over $\operatorname{Spec} B\left[\frac{1}{\zeta}\right]$, let $\tau^{\prime \prime}: \sigma^{*} \mathcal{G}_{L}^{\prime \prime} \stackrel{\sim}{\longrightarrow} \mathcal{G}_{L}^{\prime \prime}$ be an isomorphism of $L^{+} G$-torsors and let $u_{L}^{\prime \prime}: L \mathcal{G}_{L}^{\prime \prime} \stackrel{\sim}{\longrightarrow} \mathcal{G}_{B\left[\frac{1}{\zeta}\right]}$ be an isomorphism of $L G$-torsors over $\operatorname{Spec} B\left[\frac{1}{\zeta}\right]$ satisfying $u_{L}^{\prime \prime} \circ \tau^{\prime \prime}=\tau_{\mathcal{G}} \circ \sigma^{*} u_{L}^{\prime \prime}$.

Then there is a blowing-up $Y$ of $\operatorname{Spec} B$ in a finitely generated ideal $\mathfrak{b} \subset B$ containing a power of $\zeta$, an $L^{+} G$-torsor $\mathcal{G}^{\prime \prime}$ over $Y$, an isomorphism $\tau_{\mathcal{G}^{\prime \prime}}: L_{z-\zeta} \sigma^{*} \mathcal{G}^{\prime \prime} \stackrel{\sim}{\longrightarrow} L_{z-\zeta} \mathcal{G}^{\prime \prime}$ of the associated $L_{z-\zeta} G$-torsors over $Y$ and an isomorphism $u^{\prime \prime}: L \mathcal{G}^{\prime \prime} \stackrel{\sim}{\longrightarrow} \mathcal{G}_{Y}$ of $L G$-torsors satisfying $u^{\prime \prime} \circ \tau_{\mathcal{G}^{\prime \prime}}=\tau_{\mathcal{G}} \circ \sigma^{*} u^{\prime \prime}$, such that the pullback of $\left(\mathcal{G}^{\prime \prime}, \tau_{\mathcal{G}^{\prime \prime}}, u^{\prime \prime}\right)$ to $Y \times{ }_{B} \operatorname{Spec} B\left[\frac{1}{\zeta}\right]=$ 
$\operatorname{Spec} B\left[\frac{1}{\zeta}\right]$ is isomorphic to $\left(\mathcal{G}_{L}^{\prime \prime}, \tau^{\prime \prime}, u_{L}^{\prime \prime}\right)$ via an isomorphism of $L^{+} G$-torsors $h: \mathcal{G}_{B\left[\frac{1}{\zeta}\right]}^{\prime \prime} \stackrel{\sim}{\longrightarrow}$ $\mathcal{G}_{L}^{\prime \prime}$ satisfying $h \circ \tau_{\mathcal{G}^{\prime \prime}}=\tau^{\prime \prime} \circ \sigma^{*} h$ and $u^{\prime \prime}=u_{L}^{\prime \prime} \circ h$.

Moreover, if the element $\sigma^{*} \alpha \circ \tau_{\mathcal{G}}^{-1} \circ \alpha^{-1} \in G\left(\widetilde{B} \llbracket z \rrbracket\left[\frac{1}{z(z-\zeta)}\right]\right) \subset G\left(\widetilde{B}\left[\frac{1}{\zeta}\right] \llbracket z \rrbracket\left[\frac{1}{z-\zeta}\right]\right)$ maps to a point in $\hat{Z}^{\text {an }}\left(\mathrm{BSpec} \widetilde{B}\left[\frac{1}{\zeta}\right]\right)$, then $\tau_{\mathcal{G}^{\prime \prime}}$ is bounded by $\hat{Z}^{-1}$ and $\underline{\mathcal{G}}^{\prime \prime}=\left(\mathcal{G}^{\prime \prime}, \tau_{\mathcal{G}^{\prime \prime}}\right)$ is a local shtuka over $Y$ bounded by $\hat{Z}^{-1}$ in the sense of Remark $7 . \%$.

Proof. We consider the functor $\underline{\mathcal{M}}_{\mathcal{G}}$ on Spec $B$-schemes classifying quasi-isogenies of (unbounded) local $\mathcal{G}$-shtukas to $\underline{\mathcal{G}}:=\underline{=}\left(\mathcal{G}, \tau_{\mathcal{G}}\right)$, which on affine $B$-schemes $S=\operatorname{Spec} R$ is defined by

$\underline{\mathcal{M}}_{\underline{\mathcal{G}}}(S):=\left\{\right.$ Isomorphism classes of $\left(\mathcal{G}^{\prime \prime}, \tau_{\mathcal{G}^{\prime \prime}}, u^{\prime \prime}\right)$ : where $\mathcal{G}^{\prime \prime}$ is an $L^{+} G$-torsor over $R$,

where $\tau_{\mathcal{G}^{\prime \prime}}: L_{z(z-\zeta)} \sigma^{*} \mathcal{G}^{\prime \prime} \stackrel{\sim}{\longrightarrow} L_{z(z-\zeta)} \mathcal{G}^{\prime \prime}$ is an isomorphism of the associated $L_{z(z-\zeta)} G$-torsors and $u^{\prime \prime}: L \mathcal{G}^{\prime \prime} \stackrel{\sim}{\longrightarrow} \times_{B} S$ is an isomorphism of $L G$-torsors, with $\left.\tau_{\mathcal{G}} \circ \sigma^{*} u^{\prime \prime}=u^{\prime \prime} \circ \tau_{\mathcal{G}^{\prime \prime}}\right\}$.

Here $\left(\mathcal{G}^{\prime \prime}, \tau_{\mathcal{G}^{\prime \prime}}, u^{\prime \prime}\right)$ and $\left(\mathcal{G}^{\prime}, \tau_{\mathcal{G}^{\prime}}, u^{\prime}\right)$ are isomorphic if there is an isomorphism $h: \mathcal{G}^{\prime \prime} \stackrel{\sim}{\longrightarrow} \mathcal{G}^{\prime}$ of $L^{+} G$-torsors satisfying $h \circ \tau_{\mathcal{G}^{\prime \prime}}=\tau_{\mathcal{G}^{\prime}} \circ \sigma^{*} h$ and $u^{\prime \prime}=u^{\prime} \circ h$.

The functor $\underline{\mathcal{M}}_{\underline{\mathcal{G}}}$ is representable by an ind-projective ind-scheme over $\operatorname{Spec} B$ as follows. We consider its base change $\underline{\mathcal{M}}_{\underline{\mathcal{G}}} \otimes_{B} \widetilde{B}$ and fix a trivialisation $\alpha: \mathcal{G} \otimes_{B} \widetilde{B} \stackrel{\sim}{\longrightarrow} L G_{\widetilde{B}}$. Over a $\widetilde{B}$-algebra $R$ the data $\left(\mathcal{G}^{\prime \prime}, \alpha \circ u^{\prime \prime}\right)$ are represented by the ind-projective ind-scheme $\mathcal{F} \ell_{G} \widehat{\times}_{\mathbb{F}_{q}} \operatorname{Spec} \widetilde{B}$ over $\operatorname{Spec} \widetilde{B}$. Indeed, over an étale covering $\operatorname{Spec} \widetilde{R}$ of $\operatorname{Spec} R$ the $L^{+} G-$ torsor $\mathcal{G}^{\prime \prime}$ can be trivialised by an isomorphism $\beta: \mathcal{G}_{\widetilde{R}}^{\prime \prime} \stackrel{\sim}{\longrightarrow}\left(L^{+} G\right)_{\widetilde{R}}$ and then $\alpha \circ u^{\prime \prime} \circ \beta^{-1}$ yields an $\widetilde{R}$-valued point of $\mathcal{F} \ell_{G}$ that is independent of all choices and descends to an $R$-valued point of $\mathcal{F} \ell_{G}$; see [56, Theorem 6.2] or [4, Theorem 4.4] for more details and for the inverse construction. Over $\operatorname{Spec} \widetilde{R}$, also

$$
\beta \circ \tau_{\mathcal{G}^{\prime \prime}} \circ \sigma^{*} \beta^{-1}=\beta \circ\left(u^{\prime \prime}\right)^{-1} \circ \tau_{G} \circ \sigma^{*} u^{\prime \prime} \circ \sigma^{*} \beta^{-1} \in L_{z(z-\zeta)} G(\widetilde{R})=G\left(\widetilde{R} \llbracket z \rrbracket\left[\frac{1}{z(z-\zeta)}\right]\right)
$$

is uniquely determined by $u^{\prime \prime}$. This shows that $\underline{\mathcal{M}}_{\underline{\mathcal{G}}} \otimes_{B} \operatorname{Spec} \widetilde{B} \stackrel{\sim}{\sim} \ell_{G} \widehat{\times}_{\mathbb{F}_{q}} \operatorname{Spec} \widetilde{B}$ is an ind-projective ind-scheme over Spec $\widetilde{B}$. It descends to an ind-projective ind-scheme $\underline{\mathcal{M}}_{\underline{\mathcal{G}}}$ over Spec $B$, because $B \rightarrow \widetilde{B}$ is faithfully flat by [18, Lemma 1.6]; see [4, Theorem 4.4] for details.

The triple $\left(\mathcal{G}_{L}^{\prime \prime}, \tau^{\prime \prime}, u_{L}^{\prime \prime}\right)$ corresponds to a morphism $f: \operatorname{Spec} B\left[\frac{1}{\zeta}\right] \rightarrow \underline{\mathcal{M}}_{\underline{\mathcal{G}}}$. Because its source is quasi-compact, $f$ factors through a subscheme $\underline{\mathcal{M}}_{\underline{\mathcal{G}}}^{(N)}$ that is projective over Spec $B$; see [56, Lemma 5.4]. The scheme-theoretic closure $\Gamma$ of the graph of $f$ in $\underline{\mathcal{M}}_{\mathcal{G}}^{(N)}$ is a projective scheme over $\operatorname{Spec} B$ and the projection $\Gamma \rightarrow \operatorname{Spec} B$ is an isomorphism over $\operatorname{Spec} B\left[\frac{1}{\zeta}\right]$. By the flattening technique of Raynaud and Gruson [83, Corollaire 5.7.12], there is a blowing-up $Y$ of $\operatorname{Spec} B$ in a finitely generated ideal $\mathfrak{b} \subset B$ containing a power of $\zeta$, such that the strict transform of $\Gamma$ - that is, the closed subscheme of $\Gamma \times_{B} Y$ defined by the sheaf of ideals of $\zeta$-torsion - is isomorphic to $Y$. The morphism $Y \rightarrow \Gamma \rightarrow \underline{\mathcal{M}_{\mathcal{G}}^{(N)}}$ corresponds to an extension over $Y$ of the triple $\left(\mathcal{G}_{L}^{\prime \prime}, \tau^{\prime \prime}, u_{L}^{\prime \prime}\right)$ from $\operatorname{Spec} B\left[\frac{1}{\zeta}\right]$. This means 
that over $Y$ there is an $L^{+} G$-torsor $\mathcal{G}^{\prime \prime}$, an isomorphism of the associated $L_{z(z-\zeta)} G$ torsors $\tau_{\mathcal{G}^{\prime \prime}}: L_{z(z-\zeta)} \sigma^{*} \mathcal{G}^{\prime \prime} \stackrel{\sim}{\longrightarrow} L_{z(z-\zeta)} \mathcal{G}^{\prime \prime}$ and an isomorphism of the associated $L G$-torsors $u^{\prime \prime}: L \mathcal{G}^{\prime \prime} \stackrel{\sim}{\longrightarrow} \mathcal{G} \times{ }_{B} Y$ with $\tau_{\mathcal{G}} \circ \sigma^{*} u^{\prime \prime}=u^{\prime \prime} \circ \tau_{\mathcal{G}^{\prime \prime}}$ and over $Y \times_{B} \operatorname{Spec} B\left[\frac{1}{\zeta}\right]=\operatorname{Spec} B\left[\frac{1}{\zeta}\right]$ an isomorphism of $L^{+} G$-torsors $h: \mathcal{G}_{B\left[\frac{1}{\zeta}\right]}^{\prime \prime} \stackrel{\sim}{\longrightarrow} \mathcal{G}_{L}^{\prime \prime}$ satisfying $h \circ \tau_{\mathcal{G}^{\prime \prime}}=\tau^{\prime \prime} \circ \sigma^{*} h$ and $u^{\prime \prime}=u_{L}^{\prime \prime} \circ h$.

We claim that $\tau_{\mathcal{G}^{\prime \prime}}$ comes from an isomorphism of $L_{z-\zeta} G$-torsors $\tau_{\mathcal{G}^{\prime \prime}}: L_{z-\zeta} \sigma^{*} \mathcal{G}^{\prime \prime} \stackrel{\sim}{\longrightarrow}$ $L_{z-\zeta} \mathcal{G}^{\prime \prime}$. We choose a trivialisation of $\underline{\mathcal{G}}^{\prime \prime}$ over an étale covering $\widetilde{Y}$ of $Y \times_{\operatorname{Spec} B} \operatorname{Spec} \widetilde{B}$ and write the Frobenius $\tau_{\mathcal{G}^{\prime \prime}}$ of $\underline{\mathcal{G}}^{\prime \prime}$ as an element $\tau_{\mathcal{G}^{\prime \prime}} \in G\left(\mathcal{O}_{\widetilde{Y}} \llbracket z \rrbracket\left[\frac{1}{z(z-\zeta)}\right]\right)$. Our claim means that $\tau_{\mathcal{G}^{\prime \prime}}$ actually lies in $G\left(\mathcal{O}_{\widetilde{Y}} \llbracket z \rrbracket\left[\frac{1}{z-\zeta}\right]\right)$. To prove the claim, we choose a faithful representation $\rho: G \hookrightarrow \mathrm{SL}_{r}$ and we consider the matrix entries $g_{i j}$ of $\rho\left(\tau_{\mathcal{G}^{\prime \prime}}\right)$ that satisfy $(z-\zeta)^{m} g_{i j} \in \mathcal{O}_{\widetilde{Y}} \llbracket z \rrbracket\left[\frac{1}{z}\right]$ for an appropriate power $m$. Because $\tau_{\mathcal{G}^{\prime \prime}}$ differs from $\tau^{\prime \prime}$ over $\widetilde{Y}_{L}:=\widetilde{Y} \otimes_{\mathcal{O}_{L}} L$ by the isomorphism $h$ of $L^{+} G$-torsors, we see that $(z-\zeta)^{m} g_{i j} \in \mathcal{O}_{\widetilde{Y}_{L}} \llbracket z \rrbracket$. Because the intersection of $\mathcal{O}_{\widetilde{Y}} \llbracket z \rrbracket\left[\frac{1}{z}\right]$ and $\mathcal{O}_{\widetilde{Y}_{L}} \llbracket z \rrbracket$ in $\mathcal{O}_{\widetilde{Y}_{L}} \llbracket z \rrbracket\left[\frac{1}{z}\right]$ equals $\mathcal{O}_{\widetilde{Y}} \llbracket z \rrbracket$, this shows that $(z-\zeta)^{m} g_{i j} \in \mathcal{O}_{\widetilde{Y}} \llbracket z \rrbracket$. In particular, $g_{i j} \in \mathcal{O}_{\widetilde{Y}} \llbracket z \rrbracket\left[\frac{1}{z-\zeta}\right]$, and this proves our claim.

It remains to show that $\underline{\mathcal{G}}^{\prime \prime}$ is bounded by $\hat{Z}^{-1}$ under the additional assumptions on $\tau_{\mathcal{G}}$. By [18, Propositions 2.1 and 1.3], the $\zeta$-adic completion $\mathcal{Y}$ of $Y$ is the admissible formal blowing-up of $\operatorname{Spf} B$ in the ideal $\mathfrak{b}$. By Proposition 2.6(a), there is an integer $n$

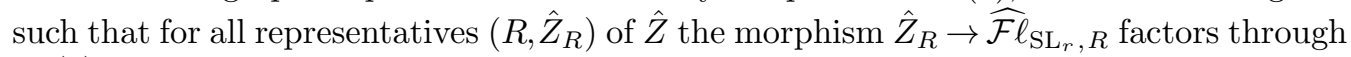
$\widehat{\mathcal{F}}_{\mathrm{SL}_{r}, R}^{(n)}$. By enlarging $n$, we may assume that the morphism $\tilde{f}: \tilde{\mathcal{Y}} \rightarrow \widehat{\mathcal{F}}_{G, R_{\hat{Z}}} \rightarrow \widehat{\mathcal{F}}_{\mathrm{SL}_{r}, R_{\hat{Z}}}$ defined by $\tau_{\mathcal{G}^{\prime \prime}}^{-1}$ factors through $\widehat{\mathcal{F}}_{\mathrm{SL}_{r}}^{(n)}, R_{\hat{Z}}$. Let $\left(R, \hat{Z}_{R}\right)$ be such a representative and set $\widetilde{\mathcal{Y}}_{R}:=\tilde{\mathcal{Y}} \widehat{\times}_{R_{\hat{Z}}}$ Spf $R$. Thus, $\tilde{f} \widehat{\otimes} \operatorname{id}_{R}: \widetilde{\mathcal{Y}}_{R} \rightarrow \widehat{\mathcal{F}}_{G, R}^{(n)}:=\widehat{\mathcal{F}}_{G, R} \widehat{\times}_{\widehat{\mathcal{F}}_{\mathrm{SL}_{r}, R}} \widehat{\mathcal{F}}_{\mathrm{SL}_{r}, R}^{(n)}$ and $\hat{Z}_{R}$ is a closed formal subscheme of $\widehat{\mathcal{F l}}_{G, R}^{(n)}$, defined by a sheaf of ideals a on $\widehat{\mathcal{F l}}_{G, R}^{(n)}$. We must show that $\left(\tilde{f} \widehat{\otimes} \operatorname{id}_{R}\right)^{*} \mathfrak{a}=(0)$. The associated morphism of $\operatorname{Frac}(R)$-analytic spaces $\left(\tilde{f} \widehat{\otimes} \operatorname{id}_{R}\right)^{\text {an }}:\left(\tilde{\mathcal{Y}}_{R}\right)^{\text {an }} \rightarrow\left(\widehat{\mathcal{F}}_{G, R}^{(n)}\right)^{\text {an }}$ is given by $\tau_{\mathcal{G}^{\prime \prime}}^{-1}=\left(\sigma^{*} u^{\prime \prime}\right)^{-1} \circ \tau_{\mathcal{G}}^{-1} \circ u^{\prime \prime}$ and factors through $\hat{Z}_{R}^{\text {an }}$, because $\sigma^{*} \alpha \circ \tau_{\mathcal{G}}^{-1} \circ \alpha^{-1} \in \hat{Z}_{R}^{\text {an }}\left(\widetilde{\mathcal{Y}}^{\text {an }}\right)$, as well as $\alpha \circ u^{\prime \prime}, \sigma^{*}\left(\alpha \circ u^{\prime \prime}\right)^{-1} \in$ $G\left(\mathcal{O}_{\widetilde{\mathcal{Y}}^{\text {an }}} \llbracket z-\zeta \rrbracket\right)$, and $\hat{Z}_{R}^{\text {an }}$ is invariant under multiplication with $G\left(\mathcal{O}_{\widetilde{\mathcal{Y}}^{\text {an }}} \llbracket z-\zeta \rrbracket\right)$ on the left. This implies $\left(\tilde{f} \widehat{\otimes} \operatorname{id}_{R}\right)^{\text {an* }} \mathfrak{a}=(0)$ on $\left(\widetilde{\mathcal{Y}}_{R}\right)^{\text {an }}$ and because $\mathcal{O}_{\widetilde{\mathcal{Y}}_{R}} \subset \mathcal{O}_{\left(\widetilde{\mathcal{Y}}_{R}\right)^{\text {an }}}$, we obtain $\left(\tilde{f} \widehat{\otimes} \operatorname{id}_{R}\right)^{*} \mathfrak{a}=(0)$ on $\tilde{\mathcal{Y}}_{R}$. Therefore, the morphism $\widetilde{\mathcal{Y}}_{R} \rightarrow \widehat{\mathcal{F}}_{G, R}^{(n)}$ given by $\tau_{\mathcal{G}^{\prime \prime}}^{-1}$ factors through the closed formal subscheme $\hat{Z}_{R}$. By Definition 2.2(d) and Remark 2.3(c), this means that $\tau_{\mathcal{G}^{\prime \prime}}^{-1}$ is bounded by $\hat{Z}$ and $\underline{\mathcal{G}}^{\prime \prime}$ is bounded by $\hat{Z}^{-1}$.

To define $\breve{\mathcal{M}}^{K}$ for all compact open subgroups $K \subset G\left(\mathbb{F}_{q}((z))\right)$, we proceed slightly differently than Rapoport and Zink [80, 5.34] and instead use the following definition and corollary. For a comparison with [80, 5.34], see Remark 7.14.

Definition 7.10. Let $X$ be a connected affinoid strictly $L$-analytic space with geometric base point $\bar{x}$, and let $K \subset G\left(\mathbb{F}_{q}((z))\right)$ be a compact open subgroup. Consider the category of triples $(\underline{\mathcal{G}}, \bar{\delta}, \eta K)$ where $(\underline{\mathcal{G}}, \bar{\delta}) \in \breve{\mathcal{M}}_{\underline{\mathbb{G}}_{0}}^{\hat{Z}^{-1}}(\mathcal{X})$ for an admissible formal model $\mathcal{X}$ of $X$ and $\eta K \in \operatorname{Triv}_{\mathcal{G}, \bar{x}}\left(\mathbb{F}_{q}((z))\right) / K$ is a rational $K$-level structure on $\underline{\mathcal{G}}$ over $X$. Morphisms between two such triples $\left(\underline{\mathcal{G}}_{1}, \bar{\delta}_{1}, \eta_{1} K\right)$ and $\left(\underline{\mathcal{G}}_{2}, \bar{\delta}_{2}, \eta_{2} K\right)$ over admissible formal models 
$\mathcal{X}_{1}$, respectively $\mathcal{X}_{2}$, are quasi-isogenies $u: \underline{\mathcal{G}}_{1} \rightarrow \underline{\mathcal{G}}_{2}$ as in Remark 7.7 over a model $\tilde{\mathcal{X}}$ dominating both $\mathcal{X}_{i}$ with $\bar{\delta}_{2} \circ(u \bmod \zeta)=\bar{\delta}_{1}$ such that $\check{V}_{u, \bar{x}} \circ \eta_{1} K=\eta_{2} K$. In particular, all morphisms are isomorphisms and by rigidity of quasi-isogenies [4, Proposition 2.11], all Hom sets contain at most one element.

For $K \subset G\left(\mathbb{F}_{q} \llbracket z \rrbracket\right)$ we consider over $\breve{\mathcal{M}}^{K}$ the triple $\left(\underline{\mathcal{G}}^{\text {univ }}, \bar{\delta}^{\text {univ }}, \eta^{\text {univ }} K\right)$, where $\eta^{\text {univ }} K$ is the rational $K$-level structure on $\underline{\mathcal{G}}^{\text {univ }}$ induced from the universal integral $K$-level structure on $\underline{\mathcal{G}}^{\text {univ }}$ via the inclusion $\operatorname{Triv}_{\underline{\mathcal{G}}, \bar{x}}\left(\mathbb{F}_{q} \llbracket z \rrbracket\right) \rightarrow \operatorname{Triv}_{\underline{\mathcal{G}}, \bar{x}}\left(\mathbb{F}_{q}((z))\right)$.

Corollary 7.11. Let $\breve{\mathcal{M}}=\left(\breve{\mathcal{M}}_{\underline{\mathbb{G}}_{0}}^{\hat{Z}^{-1}}\right)^{\text {an }}$ and for every open subgroup $K \subset G\left(\mathbb{F}_{q} \llbracket z \rrbracket\right)$ let $\breve{\mathcal{M}}^{K}$ be the finite étale covering space from Definition 7.4 parametrising integral K-level structures on the universal local G-shtuka $\underline{\mathcal{G}}^{\text {univ }}$ over $\breve{\mathcal{M}}$ from Remark 7.7. Then $\breve{\mathcal{M}}^{K}$ also parametrises isomorphism classes of triples $(\underline{\mathcal{G}}, \bar{\delta}, \eta K)$ in the sense of Definition $\% .10$.

Proof. Let $X$ be a connected affinoid strictly $L$-analytic space with geometric base point $\bar{x}$, and let $(\underline{\mathcal{G}}, \bar{\delta}, \eta K)$ be a triple over $X$, where $(\underline{\mathcal{G}}, \bar{\delta}) \in \breve{\mathcal{M}}_{\mathbb{G}_{0}}^{\hat{Z}^{-1}}(\mathcal{X})$ for an admissible formal model $\mathcal{X}$ of $X$, and $\eta K$ is a rational $K$-level structure on $\underline{\mathcal{G}}$ over $X$. We may assume that $\mathcal{X}=\operatorname{Spf} B$ is affine. By Proposition 7.8 there is an admissible formal blowing-up $\mathcal{Y} \rightarrow \mathcal{X}$ and a $\left(\underline{\mathcal{G}}^{\prime \prime}, \bar{\delta}^{\prime \prime}\right) \in \breve{\mathcal{M}}_{\underline{\underline{G}}_{0}}^{\hat{\boldsymbol{Z}}^{-1}}(\mathcal{Y})$ with $\breve{\pi}(\underline{\mathcal{G}}, \bar{\delta})=\breve{\pi}\left(\underline{\mathcal{G}}^{\prime \prime}, \bar{\delta}^{\prime \prime}\right)$ and an isogeny $u^{\prime \prime}: \underline{\mathcal{G}} \rightarrow \underline{\mathcal{G}}^{\prime \prime}$ over $\mathcal{Y}$ lifting $\left(\bar{\delta}^{\prime \prime}\right)^{-1} \circ \bar{\delta}$ such that $\check{V}_{u^{\prime \prime}, \bar{x}} \circ \eta \in \operatorname{Triv}_{\mathcal{G}^{\prime \prime}, \bar{x}}\left(\mathbb{F}_{q} \llbracket z \rrbracket\right)$. The pair $\left(\underline{\mathcal{G}}^{\prime \prime}, \bar{\delta}^{\prime \prime}\right)$ induces a morphism of $\breve{E}$-analytic spaces $X=\mathcal{Y}^{\text {an }} \rightarrow \breve{\mathcal{M}}$ that is independent of the admissible formal blowingup $\mathcal{Y}$. By Proposition 7.5(a) the integral $K$-level structure $\left(\check{V}_{u^{\prime \prime}, \bar{x}} \circ \eta\right) K$ on $\underline{\mathcal{G}}^{\prime \prime}$ defines a uniquely determined $\breve{\mathcal{M}}$-morphism $f: X \rightarrow \breve{\mathcal{M}}^{K}$ such that $\left(f^{*} \underline{\mathcal{G}}^{\text {univ }}, f^{*} \bar{\delta}^{\text {univ }}, \bar{f}^{*} \eta^{\text {univ }} K\right)=$ $\left(\underline{\mathcal{G}}^{\prime \prime}, \bar{\delta}^{\prime \prime},\left(\check{V}_{u^{\prime \prime}, \bar{x}} \circ \eta\right) K\right) \cong(\underline{\mathcal{G}}, \bar{\delta}, \eta K)$.

Definition 7.12. Let $K \subset G\left(\mathbb{F}_{q}((z))\right)$ be a compact open subgroup. Let $K^{\prime} \subset K$ be a normal subgroup of finite index with $K^{\prime} \subset G\left(\mathbb{F}_{q} \llbracket z \rrbracket\right)$. (Such a subgroup exists because $K \cap G\left(\mathbb{F}_{q} \llbracket z \rrbracket\right) \subset K$ is of finite index due to the openness of $G\left(\mathbb{F}_{q} \llbracket z \rrbracket\right)$ and the compactness of $K$.) Then we define $\breve{\mathcal{M}}^{K}$ as the $\breve{E}$-analytic space that is the quotient of $\breve{\mathcal{M}}^{K^{\prime}}$ by the finite group $K / K^{\prime}$; see [7, Proposition 2.1.14(ii)] and [10, Lemma 4]. Here $g K^{\prime} \in$ $K / K^{\prime}$ acts on $\breve{\mathcal{M}}^{K^{\prime}}$ by sending the universal triple $\left(\underline{\mathcal{G}}^{\text {univ }}, \bar{\delta}^{\text {univ }}, \eta^{\text {univ }} K^{\prime}\right)$ over $\breve{\mathcal{M}}^{K^{\prime}}$ from Corollary 7.11 to the triple $\left(\underline{\mathcal{G}}^{\text {univ }}, \bar{\delta}^{\text {univ }}, \eta^{\text {univ }} g K^{\prime}\right)$. By Remark 7.14 , this means that $g K^{\prime} \in$ $K / K^{\prime}$ acts on $\breve{\mathcal{M}}^{K^{\prime}}$ as the Hecke correspondence $\iota(g)_{K^{\prime}}$. In particular, $\breve{\mathcal{M}}^{K_{0}}=\left(\breve{\mathcal{M}}_{\mathbb{G}_{0}}^{\hat{Z}^{-1}}\right)^{\text {an }}$ for $K_{0}=G\left(\mathbb{F}_{q} \llbracket z \rrbracket\right)$. We denote by $\left(\underline{\mathcal{G}}^{\text {univ }}, \bar{\delta}^{\text {univ }}, \eta^{\text {univ }} K\right)$ the triple over $\breve{\mathcal{M}}^{K}$ induced by the universal triple $\left(\underline{\mathcal{G}}^{\text {univ }}, \bar{\delta}^{\text {univ }}, \eta^{\text {univ }} K^{\prime}\right)$ over $\breve{\mathcal{M}}^{K^{\prime}}$. It is universal by the following corollary.

By Proposition 7.5(c), the definition of $\breve{\mathcal{M}}^{K}$ is independent of the normal subgroup $K^{\prime} \subset K$ and Proposition 7.5(c) continues to hold in this more general setting. We will see in Theorem 8.1 that $\breve{\mathcal{M}}^{K}$ always is a strictly $\breve{E}$-analytic space.

Corollary 7.13. If $K \subset G\left(\mathbb{F}_{q}((z))\right)$ is any compact open subgroup then $\breve{\mathcal{M}}^{K}$ is an $\breve{E}$ analytic space that is separated and partially proper over $\breve{E}$ and parametrises isomorphism classes of triples $(\underline{\mathcal{G}}, \bar{\delta}, \eta K)$ in the sense of Definition $\% .10$.

Proof. That $\breve{\mathcal{M}}^{K}$ is separated and partially proper over $\breve{E}$ follows from Lemma 6.11 and [63, Lemma 1.10.17 (iv), (vii)]. Let $X$ be a connected affinoid strictly $L$-analytic 
space with geometric base point $\bar{x}$, and let $(\underline{\mathcal{G}}, \bar{\delta}, \eta K)$ be a triple over $X$ where $(\underline{\mathcal{G}}, \bar{\delta}) \in$ $\breve{\mathcal{M}}_{\mathbb{G}_{0}}^{\hat{Z}^{-1}}(\mathcal{X})$ for an admissible formal model $\mathcal{X}$ of $X$ and where $\eta K \in \operatorname{Triv}_{\underline{\mathcal{G}}, \bar{x}}\left(\mathbb{F}_{q}((z))\right) / K$ is a rational $K$-level structure on $\underline{\mathcal{G}}$ over $X$. Let $K^{\prime} \subset K$ be a normal subgroup of finite index with $K^{\prime} \subset G\left(\mathbb{F}_{q} \llbracket z \rrbracket\right)$. Consider the étale covering space $X^{\prime} \rightarrow X$ corresponding to the $\pi_{1}^{\text {ét }}(X, \bar{x})$-set $\left\{\eta^{\prime} K^{\prime} \in \operatorname{Triv}_{\mathcal{G}, \bar{x}}\left(\mathbb{F}_{q}((z))\right) / K^{\prime}: \eta^{\prime} K=\eta K\right\}$ that is isomorphic to $K / K^{\prime}$ under the maps $\eta^{\prime} K^{\prime} \mapsto \eta^{-1} \eta^{\prime} K^{\prime}$ and $\eta g K^{\prime} \leftrightarrow g K^{\prime}$. In particular, $X^{\prime} \rightarrow X$ is a $K / K^{\prime}$ torsor. By Corollary 7.11 there is a uniquely determined $\breve{E}$-morphism $X^{\prime} \rightarrow \breve{\mathcal{M}}^{K^{\prime}}$ which is equivariant for the action of $K / K^{\prime}$ and therefore descends to a uniquely determined $\breve{E}$-morphism $X \rightarrow \breve{\mathcal{M}}^{K}$.

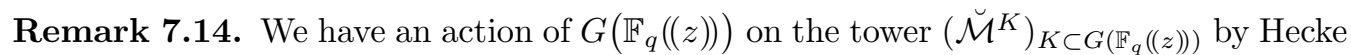
correspondences defined as follows. Let $g \in G\left(\mathbb{F}_{q}((z))\right)$ and let $K$ be a compact open subgroup of $G\left(\mathbb{F}_{q}((z))\right)$. Then $g$ induces an isomorphism

$$
\iota(g)_{K}: \breve{\mathcal{M}}^{K} \stackrel{\sim}{\longrightarrow} \breve{\mathcal{M}}^{g^{-1} K g}
$$

by sending the universal triple $\left(\underline{\mathcal{G}}^{\text {univ }}, \bar{\delta}^{\text {univ }}, \eta^{\text {univ }} K\right)$ over $\breve{\mathcal{M}}^{K}$ from Definition 7.12 to the triple $\left(\underline{\mathcal{G}}^{\text {univ }}, \bar{\delta}^{\text {univ }}, \eta^{\text {univ }} K g=\eta^{\text {univ }} g\left(g^{-1} K g\right)\right)$. The morphisms $\iota(g)$ are compatible with the group structure on $G\left(\mathbb{F}_{q}((z))\right)$; that is, they satisfy $\iota(g h)_{K}=\iota(g)_{h^{-1} K h} \circ \iota(h)_{K}$ for all $g, h \in G\left(\mathbb{F}_{q}((z))\right)$ and all $K$. They are also compatible with the projection maps $\breve{\pi}_{K, K^{\prime}}$; that is, $\iota(g)_{K} \circ \breve{\pi}_{K, K^{\prime}}=\breve{\pi}_{g^{-1}} K g, g^{-1} K^{\prime} g \circ \iota(g)_{K^{\prime}}$.

If both $K$ and $g^{-1} K g$ are contained in $G\left(\mathbb{F}_{q} \llbracket z \rrbracket\right)$, we can translate the definition of $\iota(g)_{K}$ in terms of integral $K$-level structures by inspecting the proof of Corollary 7.11. Namely, we start with the universal integral $K$-level structure $\eta^{\text {univ }} K \in \operatorname{Triv}_{\underline{\mathcal{G}}^{\text {univ }}, \bar{x}}\left(\mathbb{F}_{q} \llbracket z \rrbracket\right) / K$ on $\underline{\mathcal{G}}^{\text {univ }}$ over $\breve{\mathcal{M}}^{K}$. The rational $g^{-1} K g$-level structure $\eta^{\text {univ }} K g=\eta^{\text {univ }} g\left(g^{-1} K g\right)$ yields a pair $\left(\underline{\mathcal{G}}^{\prime \prime}, \bar{\delta}^{\prime \prime}\right) \in \breve{\mathcal{M}}_{\mathbb{\mathbb { I }}_{0}}^{\hat{Z}^{-1}}(\mathcal{Y})$ for an admissible formal blowing-up $\mathcal{Y} \rightarrow \mathcal{X}$ with $\breve{\pi}\left(\underline{\mathcal{G}}^{\text {univ }}, \bar{\delta}^{\text {univ }}\right)=$ $\breve{\pi}\left(\underline{\mathcal{G}}^{\prime \prime}, \bar{\delta}^{\prime \prime}\right)$ and $\check{V}_{u^{\prime \prime}, \bar{x}} \circ \eta^{\text {univ }} g \in \operatorname{Triv}_{\underline{\mathcal{G}}^{\prime \prime}, \bar{x}}\left(\mathbb{F}_{q} \llbracket z \rrbracket\right)$, where $u^{\prime \prime}$ is the unique lift of $\left(\bar{\delta}^{\prime \prime}\right)^{-1} \circ \bar{\delta}^{\text {univ }}$. Then the morphism $\iota(g)_{K}$ is given by

$$
\iota(g)_{K}:\left(\underline{\mathcal{G}}^{\text {univ }}, \bar{\delta}^{\text {univ }}, \eta^{\text {univ }} K\right) \longmapsto\left(\underline{\mathcal{G}}^{\prime \prime}, \bar{\delta}^{\prime \prime},\left(\check{V}_{u^{\prime \prime}, \bar{x}} \circ \eta^{\text {univ }} g\right) g^{-1} K g\right) .
$$

This shows that the definition of $\iota(g)_{K}$ in this case and therefore the definitions of $\breve{\mathcal{M}}^{K}$, $\breve{\pi}_{K, K^{\prime}}$ and $\iota(g)_{K}$ for all $K$ coincide with the definitions analogous to [80, 5.34].

Although it is not explicitly stated in [80], the analogue of Corollary 7.13 also holds in their setup, because it can be deduced from the existence of the $\iota(g)_{K}$ as follows. If $(\underline{\mathcal{G}}, \bar{\delta}) \in \breve{\mathcal{M}}_{\underline{\underline{G}}_{0}}^{\hat{Z}^{-1}}(\mathcal{X})$ and $\eta K$ with $\eta \in \operatorname{Triv}_{\underline{\mathcal{G}}, \bar{x}}\left(\mathbb{F}_{q}((z))\right)$ is a rational $K$-level structure

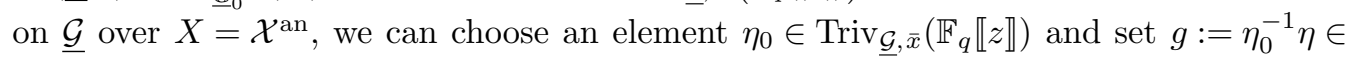
$\operatorname{Aut}^{\otimes}\left(\omega^{\circ}\right)=G\left(\mathbb{F}_{q}((z))\right)$. Then $\eta K g^{-1}=\eta_{0}\left(g K g^{-1}\right)$ is an integral $g K g^{-1}$-level structure on $\underline{\mathcal{G}}$ and defines a uniquely determined $\breve{E}$-morphism $X \rightarrow \breve{\mathcal{M}}^{g K g^{-1}}$. Composing with $\iota(g)_{g K g^{-1}}$ produces the desired $\breve{E}$-morphism $X \rightarrow \breve{\mathcal{M}}^{K}$.

Proposition 7.15. The period morphism $\breve{\pi}$ induces compatible morphisms $\breve{\pi}_{K}: \breve{\mathcal{M}}^{K} \rightarrow$ $\breve{\mathcal{H}}_{G, \hat{Z}}^{\text {an }}$ for all compact open subgroups $K \subset G\left(\mathbb{F}_{q}((z))\right)$. In terms of Corollary 7.13, it has the form $\breve{\pi}_{K}:\left(\underline{\mathcal{G}}^{\text {univ }}, \bar{\delta}^{\text {univ }}, \eta^{\text {univ }} K\right) \mapsto \breve{\pi}\left(\underline{\mathcal{G}}^{\text {univ }}, \bar{\delta}^{\text {univ }}\right)$ where $\eta^{\text {univ }} K$ is the universal 
(integral or) rational K-level structure on $\underline{\mathcal{G}}^{\text {univ }}$. These morphisms commute with the Hecke correspondences in the sense that $\breve{\pi}_{g^{-1} K g} \circ \iota(g)_{K}=\breve{\pi}_{K}$ for all $g$ and $K$.

Proof. To construct $\breve{\pi}_{K}$, we choose a normal subgroup $K^{\prime} \subset K$ of finite index with $K^{\prime} \subset G\left(\mathbb{F}_{q} \llbracket z \rrbracket\right)$. We let $\breve{\pi}_{K^{\prime}}:=\breve{\pi} \circ \breve{\pi}_{G\left(\mathbb{F}_{q} \llbracket z \rrbracket\right), K^{\prime}}: \breve{\mathcal{M}}^{K^{\prime}} \rightarrow \breve{\mathcal{H}}_{G, \hat{Z}}^{\text {an }}$. It has the given form. If $g \in$ $G\left(\mathbb{F}_{q}((z))\right)$ satisfies $g^{-1} K^{\prime} g \subset G\left(\mathbb{F}_{q} \llbracket z \rrbracket\right)$, we see from the description of $\iota(g)_{K^{\prime}}$ in $(7.6)$ that $\breve{\pi}_{g^{-1} K^{\prime} g} \circ \iota(g)_{K^{\prime}}=\breve{\pi}_{K^{\prime}}$. In particular, if $g \in K$, then $\breve{\pi}_{K^{\prime}}$ is $K / K^{\prime}$-invariant. Therefore, $\breve{\pi}_{K^{\prime}}$ descends to a morphism $\breve{\pi}_{K}: \breve{\mathcal{M}}^{K} \rightarrow \breve{\mathcal{H}}_{G, \hat{Z}}^{\text {an }}$ that has the given form. By Proposition $7.5(\mathrm{c})$, the definition of $\breve{\pi}_{K}$ is independent of the chosen $K^{\prime}$ and satisfies $\breve{\pi}_{K} \circ \breve{\pi}_{K, \widetilde{K}}=\breve{\pi}_{\widetilde{K}}$ for all compact open subgroups $\widetilde{K} \subset K \subset G\left(\mathbb{F}_{q}((z))\right)$. From this $\breve{\pi}_{g^{-1} K g} \circ \iota(g)_{K}=\breve{\pi}_{K}$ also follows.

The following result is the analogue of [80, Proposition 5.37] and can be proved in the same way. However, we give a different proof using Corollary 7.13.

Proposition 7.16. Let $K_{1}, K_{2} \subset G\left(\mathbb{F}_{q}((z))\right)$ be compact open subgroups and let $\Omega$ be an algebraically closed complete extension of $\breve{E}$. Then two points $x_{1} \in \breve{M}^{K_{1}}(\Omega)$ and $x_{2} \in$ $\breve{\mathcal{M}}^{K_{2}}(\Omega)$ satisfy $\breve{\pi}_{K_{1}}\left(x_{1}\right)=\breve{\pi}_{K_{2}}\left(x_{2}\right)$ if and only if they are mapped to each other under a Hecke correspondence; that is, if there is a $g \in G\left(\mathbb{F}_{q}((z))\right)$ and a $y \in \breve{\mathcal{M}}^{K_{1} \cap g K_{2} g^{-1}}(\Omega)$ with $\breve{\pi}_{K_{1}, K_{1} \cap g K_{2} g^{-1}}(y)=x_{1}$ and $\breve{\pi}_{K_{2}, g^{-1} K_{1} g \cap K_{2}} \circ \iota(g)_{K_{1} \cap g K_{2} g^{-1}}(y)=x_{2}$. In particular, the geometric fibres of $\breve{\pi}_{K}$ are (noncanonically) isomorphic to $G\left(\mathbb{F}_{q}((z))\right) / K$.

Proof. Because one direction was proved in Proposition 7.15, we now assume $\breve{\pi}_{K_{1}}\left(x_{1}\right)=$ $\breve{\pi}_{K_{2}}\left(x_{2}\right)$. In terms of Corollary 7.13 , let $x_{i}$ correspond to the triple $\left(\underline{\mathcal{G}}_{i}, \bar{\delta}_{i}, \eta_{i} K_{i}\right)$. Because $\Omega$ is algebraically closed, we may choose representatives $\eta_{i} \in \operatorname{Triv}_{\mathcal{G}_{i}}$, BSpec $(\Omega)\left(\mathbb{F}_{q}((z))\right)$ of $\eta_{i} K_{i}$. By the description of $\breve{\pi}_{K}$ in Proposition 7.15 , we have $\breve{\pi}\left(\underline{\mathcal{G}}_{1}, \underline{\bar{\delta}}_{1}\right)=\breve{\pi}\left(\underline{\mathcal{G}}_{2}, \bar{\delta}_{2}\right)$ and so Proposition 7.8 yields a quasi-isogeny $u: \underline{\mathcal{G}}_{2} \rightarrow \underline{\mathcal{G}}_{1}$ over $\operatorname{Spec} \mathcal{O}_{\Omega}$ with $\bar{\delta}_{1} \circ(u \bmod \zeta)=$ $\bar{\delta}_{2}$. We set $\eta_{1}^{\prime}:=\check{V}_{u, \operatorname{BSpec}(\Omega)} \circ \eta_{2} \in \operatorname{Triv}_{\underline{\mathcal{G}}_{1}, \operatorname{BSpec}(\Omega)}\left(\mathbb{F}_{q}((z))\right)$. Then $x_{2}=\left(\underline{\mathcal{G}}_{2}, \bar{\delta}_{2}, \eta_{2} K_{2}\right) \cong$ $\left(\underline{\mathcal{G}}_{1}, \bar{\delta}_{1}, \eta_{1}^{\prime} K_{2}\right)$. Therefore, $g:=\eta_{1}^{-1} \eta_{1}^{\prime} \in \operatorname{Aut}^{\otimes}\left(\omega^{\circ}\right)=G\left(\mathbb{F}_{q}((z))\right)$ and $y=\left(\underline{\mathcal{G}}_{1}, \bar{\delta}_{1}, \eta_{1}\left(K_{1} \cap\right.\right.$ $\left.\left.g K_{2} g^{-1}\right)\right) \in \breve{\mathcal{M}}^{K_{1} \cap g K_{2} g^{-1}}(\Omega)$ solve the problem.

Thus, after the choice of the representative $\eta_{1}$ of $\eta_{1} K_{1}$, the bijection between $G\left(\mathbb{F}_{q}((z))\right) / K_{1}$ and the fibre of $\breve{\pi}_{K_{1}}$ over $\breve{\pi}_{K_{1}}\left(x_{1}\right)$ is given by $g K_{1} \mapsto\left(\underline{\mathcal{G}}_{1}, \bar{\delta}_{1}, \eta_{1} g K_{1}\right)$ with inverse $\eta_{1}^{-1} \eta_{1}^{\prime} K_{1} \hookleftarrow\left(\underline{\mathcal{G}}_{1}, \bar{\delta}_{1}, \eta_{1}^{\prime} K_{1}\right)$.

Remark 7.17. Finally, the action of $j \in J_{b}\left(\mathbb{F}_{q}((z))\right)$ on $\breve{\mathcal{M}}_{\mathbb{G}_{0}}^{\hat{Z}^{-1}}$ from Definition 3.1 induces actions on each of the spaces $\breve{\mathcal{M}}^{K}$ individually by

$$
j: \breve{\mathcal{M}}^{K} \longrightarrow \breve{\mathcal{M}}^{K}, \quad(\underline{\mathcal{G}}, \bar{\delta}, \eta K) \longmapsto(\underline{\mathcal{G}}, j \circ \bar{\delta}, \eta K) .
$$

In other words, the pullback $j^{*}\left(\underline{\mathcal{G}}^{\text {univ }}, \bar{\delta}^{\text {univ }}, \eta^{\text {univ }} K\right)$ of the universal triple over $\breve{\mathcal{M}}^{K}$ is isomorphic to $\left(\underline{\mathcal{G}}^{\text {univ }}, j \circ \bar{\delta}^{\text {univ }}, \eta^{\text {univ }} K\right)$ in the category of triples from Definition 7.10. Using the universal property of $\breve{\mathcal{M}}_{\underline{\mathbb{G}}_{0}}^{\hat{Z}^{-1}}$, the condition $j^{*}\left(\underline{\mathcal{G}}^{\text {univ }}, \bar{\delta}^{\text {univ }}\right) \cong\left(\underline{\mathcal{G}}^{\text {univ }}, j \circ \bar{\delta}^{\text {univ }}\right)$ shows that there is an isomorphism $\Phi_{j}: j^{*} \underline{\mathcal{G}}^{\text {univ }} \stackrel{\sim}{\longrightarrow} \underline{\mathcal{G}}^{\text {univ }}$ of local $G$-shtukas with $j^{*} \bar{\delta}^{\text {univ }}=$ $j \circ \bar{\delta}^{\text {univ }} \circ\left(\Phi_{j} \bmod \zeta\right)$. By rigidity of quasi-isogenies [4, Proposition 2.11], the isomorphism $\Phi_{j}$ is uniquely determined and a straightforward calculation shows that $\Phi_{j} \circ j^{*} \Phi_{j^{\prime}}=\Phi_{j^{\prime} j}$. 
It follows from Definition 7.10 that $j^{*} \eta^{\text {univ }} K=\left(\check{V}_{\Phi_{j}, \bar{x}}^{-1} \circ \eta^{\text {univ }}\right) K$. The $J_{b}\left(\mathbb{F}_{q}((z))\right)$-action on $\breve{\mathcal{M}}^{K}$ is compatible with the projection maps $\breve{\pi}_{K, K^{\prime}}$ and the Hecke action.

Lemma 7.18. The action of $J:=J_{b}\left(\mathbb{F}_{q}((z))\right)$ on $\breve{\mathcal{M}}_{\mathbb{\mathbb { G }}_{0}}^{\hat{Z}^{-1}}$ and the induced actions on each $\breve{\mathcal{M}}^{K}$ are continuous.

Proof. For the first assertion we have to show the following claim. Let $S \in \mathcal{N} i l p_{\mathbb{F}_{q} \llbracket \xi \rrbracket}$ be a quasi-compact scheme and $(\underline{\mathcal{G}}, \bar{\delta}) \in \breve{\mathcal{M}}_{\underline{\mathbb{G}}_{0}}^{\hat{Z}^{-1}}(S)$. We must show that there is a neighbourhood $U$ of $1 \in J$ such that for $j \in U$ we have $j(\underline{\mathcal{G}}, \bar{\delta}) \cong(\underline{\mathcal{G}}, \bar{\delta})$; that is, $\bar{\delta}^{-1} \circ j \circ \bar{\delta}$ lifts to an automorphism of $\underline{\mathcal{G}}$ over $S$.

To prove this claim, let $\delta: \underline{\mathcal{G}} \rightarrow \underline{\mathbb{G}}_{0, S}$ be the quasi-isogeny that lifts $\bar{\delta}$ by rigidity of quasi-isogenies [4, Proposition 2.11]. Let $S^{\prime} \rightarrow S$ be an étale covering that trivialises $\underline{\mathcal{G}} \cong\left(\left(L^{+} G\right)_{S^{\prime}}, A \sigma^{*}\right)$. Because $S$ is quasi-compact, there is a refinement of this covering such that $S^{\prime}=\operatorname{Spec} B$ is affine. Then $\delta$ corresponds to an element $\Delta \in L G(B)$. We fix a faithful representation $\rho: G \hookrightarrow \mathrm{SL}_{r}$ and consider the elements $\rho(\Delta)$ and $\rho\left(\Delta^{-1}\right)$ of $L \mathrm{SL}_{r}(B)=\mathrm{SL}_{r}\left(B \llbracket z \rrbracket\left[\frac{1}{z}\right]\right)$. Let $N \in \mathbb{N}_{0}$ be such that the matrices $z^{N} \cdot \rho(\Delta)$ and $z^{N} \cdot \rho\left(\Delta^{-1}\right)$ have their entries in $B \llbracket z \rrbracket$. If $\rho(j) \equiv 1 \bmod z^{2 N}$, then $\rho\left(\Delta^{-1} \cdot j \cdot \Delta\right) \in \mathrm{SL}_{r}(B \llbracket z \rrbracket)$, and hence $\Delta^{-1} \cdot j \cdot \Delta \in L^{+} G(B)$ and $\delta^{-1} \circ j \circ \delta \in \operatorname{Aut}(\underline{\mathcal{G}})$. Because the map $J \subset L G(\mathbb{F}) \stackrel{\rho}{\rightarrow} L \mathrm{SL}_{r}(\mathbb{F})$ is continuous with respect to the $z$-adic topology, the claim follows.

Because the action on $\breve{\mathcal{M}}_{\mathbb{\Xi}_{0}}^{\hat{Z}^{-1}}$ is continuous, the same holds for each $\breve{\mathcal{M}}^{K}$ by $[9$, Lemma 8.4]. Note that continuity of the $J$-action is defined as continuity of the homomorphism $J \rightarrow \mathscr{G}\left(\breve{\mathcal{M}}^{K}\right)$, where $\mathscr{G}\left(\breve{\mathcal{M}}^{K}\right)$ is the topological automorphism group defined by Berkovich $[9, \S 6]$. The topology of $\mathscr{G}\left(\breve{\mathcal{M}}^{K}\right)$ is defined via compact subspaces of $\breve{\mathcal{M}}^{K}$. Therefore, the continuity of the $J$-action on $\breve{\mathcal{M}}_{\mathbb{\mathbb { G }}_{0}}^{\hat{Z}^{-1}}$ we proved above and [9, Lemma 8.4] are applicable although $\breve{\mathcal{M}}_{\underline{\mathbb{G}}_{0}}^{\hat{Z}^{-1}}$ is not quasi-compact.

Remark 7.19. We keep the notation from Remarks 3.7 and 4.21 and let $\varepsilon: G \rightarrow G^{\prime}$ be a morphism of parahoric group schemes over $\mathbb{F}_{q} \llbracket z \rrbracket$. If $\varepsilon(\hat{Z}) \subset \hat{Z}^{\prime}$ and $\underline{\mathbb{G}}_{0}^{\prime}=\varepsilon_{*} \underline{\mathbb{G}}_{0} \cong$ $\left(\left(L^{+} G^{\prime}\right)_{\mathbb{F}}, b^{\prime} \sigma^{*}\right)$ where $\underline{\mathbb{G}}_{0} \cong\left(\left(L^{+} G\right)_{\mathbb{F}}, b \sigma^{*}\right)$ and $b^{\prime}=\varepsilon(b)$, then there is a commutative diagram of $\breve{E}$-analytic spaces

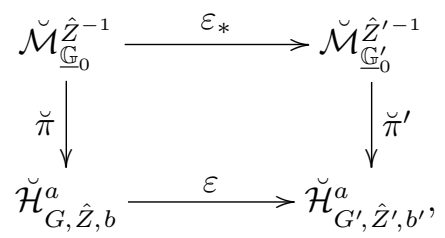

where $\breve{\pi}$ and $\breve{\pi}^{\prime}$ are the period morphisms from Definition 6.3 for $\underline{\mathbb{G}}_{0}$ and $\hat{Z}$, respectively for $\mathbb{G}_{0}^{\prime}$ and $\hat{Z}^{\prime}$, and where the horizontal morphisms $\varepsilon_{*}$ and $\varepsilon$ were described in (3.4) and (4.5). This diagram is equivariant for the action of $J_{b}^{G}$ that acts on the right column via the morphism $J_{b}^{G} \rightarrow J_{b^{\prime}}^{G^{\prime}}$ from (3.5). The diagram is indeed commutative, because in the notation of Lemma 6.2 and equation (6.4), the left morphism $\breve{\pi}$ sends $\underline{\mathcal{G}}_{\mathscr{S}^{\prime}} \cong$ $\left(\left(L^{+} G\right)_{\mathscr{S}^{\prime}}, A \sigma^{*}\right)$ to $\gamma:=\sigma^{*}(\Delta) A^{-1}=b^{-1} \Delta$ and the right morphism $\breve{\pi}^{\prime}$ sends $\varepsilon_{*}(\underline{\mathcal{G}})_{\mathscr{S}^{\prime}} \cong$ $\left(\left(L^{+} G^{\prime}\right)_{\mathscr{S}^{\prime}}, \varepsilon(A) \sigma^{*}\right)$ to $\gamma^{\prime}:=\sigma^{*}(\varepsilon(\Delta)) \varepsilon(A)^{-1}=\varepsilon(b)^{-1} \varepsilon(\Delta)=\varepsilon(\gamma)$. 
The tensor functors $\underline{\mathcal{V}}_{b}$ and $\underline{\mathcal{V}}_{b^{\prime}}$ from Theorem 5.7 are part of a commutative diagram of tensor functors

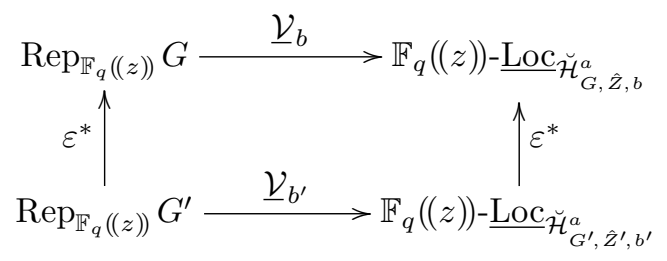

where the right vertical arrow denotes pullback of local systems of $\mathbb{F}_{q}((z))$-vector spaces along the morphism $\varepsilon: \breve{\mathcal{H}}_{G, \hat{Z}, b}^{a} \rightarrow \breve{\mathcal{H}}_{G^{\prime}, \hat{Z}^{\prime}, b^{\prime}}^{a}$ from (4.5), and the left vertical functor sends $\left(V^{\prime}, \rho^{\prime}\right)$ to $\left(V^{\prime}, \rho^{\prime} \circ \varepsilon\right)$; see Remark 4.21 .

Moreover, the Tate module functor $\check{T}_{\mathcal{G}}$ from (7.1) satisfies $\check{T}_{\varepsilon_{*} \mathcal{G}}\left(\rho^{\prime}\right)=\check{T}_{\mathcal{G}}\left(\rho^{\prime} \circ \varepsilon\right)$; that is, $\check{T}_{\varepsilon_{*} \underline{\mathcal{G}}}=\check{T}_{\underline{\mathcal{G}}} \circ \varepsilon^{*}$, and likewise $\check{V}_{\varepsilon_{*} \underline{\mathcal{G}}}=\check{V}_{\underline{\mathcal{G}}^{\circ}}{ }^{*} \varepsilon^{*}$. This defines a map of the sets from $(7.3)$

$$
\varepsilon^{*}: \operatorname{Triv}_{\underline{\mathcal{G}}, \bar{x}}(A) \longrightarrow \operatorname{Triv}_{\varepsilon_{*} \underline{\mathcal{G}}, \bar{x}}(A), \quad \eta \longmapsto \varepsilon^{*}(\eta)
$$

for both arguments $A=\mathbb{F}_{q} \llbracket z \rrbracket$ and $A=\mathbb{F}_{q}((z))$. In particular, if $K \subset G(A)$ and $K^{\prime} \subset G^{\prime}(A)$ are compact open subgroups with $\varepsilon(K) \subset K^{\prime}$, then the map $\varepsilon_{*}: \eta K \mapsto \varepsilon_{*}(\eta) K^{\prime}$ sends $K$ level structures on $\underline{\mathcal{G}}$ to $K^{\prime}$-level structures on $\varepsilon_{*} \underline{\mathcal{G}}$. This yields a commutative diagram of $\breve{E}$-analytic spaces

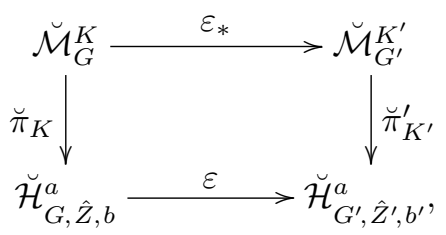

which is again $J_{b}^{G}$-equivariant. It is further equivariant for the action of $g \in G\left(\mathbb{F}_{q}((z))\right)$ that acts on the left column by the Hecke correspondence $\iota(g)_{K}$ from Remark 7.14 and on the right column by the Hecke correspondence $\iota(\varepsilon(g))_{K^{\prime}}$.

\section{The image of the period morphism}

In this section we fix a local $G$-shtuka $\underline{\mathbb{G}}_{0}=\left(\left(L^{+} G\right)_{\mathbb{F}}, b \sigma^{*}\right)$ over $\mathbb{F}$ and a bound $\hat{Z}$ with reflex ring $R_{\hat{Z}}=\kappa \llbracket \xi \rrbracket$. We set $E_{\hat{Z}}=\kappa((\xi))$ and $\breve{E}:=\mathbb{F}((\xi))$.

We will determine the image of the period morphism $\breve{\pi}$ from Definition 6.3 . This is the function field analogue of [51, Theorem 8.4], where the situation of PEL-Rapoport-Zink spaces for $p$-divisible groups was treated. Note, however, that our proof here is entirely different from [51, Theorem 8.4], because here we already constructed a tensor functor to the category of local systems in Theorem 5.7 and will use this to determine the image of $\breve{\pi}$. In [51, Theorem 8.4], the proof proceeds in the opposite direction and first determines the image of the period morphism and then constructs the local systems.

Theorem 8.1. (a) The image $\breve{\pi}\left(\left(\breve{\mathcal{M}}_{\underline{\mathbb{G}}_{0}}^{\hat{Z}^{-1}}\right)^{\text {an }}\right)$ of the period morphism $\breve{\pi}$ equals the union of the connected components of $\breve{\mathcal{H}}_{G, \hat{z}, b}^{a}$ on which there is an $\mathbb{F}_{q}((z))$ - 
rational isomorphism $\beta: \omega^{\circ} \stackrel{\sim}{\longrightarrow} \omega_{b, \bar{\gamma}}$ between the tensor functors $\omega^{\circ}$ and $\omega_{b, \bar{\gamma}}$ from Remark 5.8.

(b) The rational dual Tate module $\check{V}_{\underline{\mathcal{G}}}$ of the universal local G-shtuka $\underline{\mathcal{G}}$ over $\left(\breve{\mathcal{M}}_{\mathbb{\mathbb { G }}_{0}}^{\hat{Z}^{-1}}\right)^{\text {an }}$ descends to a tensor functor $\check{V}_{\underline{\mathcal{G}}}$ from $\operatorname{Rep}_{\mathbb{F}_{q}((z))} G$ to the category of local systems of $\mathbb{F}_{q}((z))$-vector spaces on $\breve{\pi}\left(\left(\breve{\mathcal{M}}_{\mathbb{\mathbb { G }}_{0}}^{\hat{Z}^{-1}}\right)^{\text {an }}\right)$. It carries a canonical $J_{b}\left(\mathbb{F}_{q}((z))\right)$ linearisation and is canonically $J_{b}\left(\mathbb{F}_{q}((z))\right)$-equivariantly isomorphic to the tensor functor $\underline{\mathcal{V}}_{b}$ from Theorem 5.7 .

(c) The tower of strictly $\breve{E}$-analytic spaces $\left(\breve{\mathcal{M}}^{K}\right)_{K \subset G\left(\mathbb{F}_{q}((z))\right)}$ is canonically isomorphic over $\breve{\pi}\left(\left(\breve{\mathcal{M}}_{\underline{\mathbb{G}}_{0}}^{\hat{Z}^{-1}}\right)\right.$ an $)$ in a Hecke and $J_{b}\left(\mathbb{F}_{q}((z))\right)$-equivariant way to the tower of étale

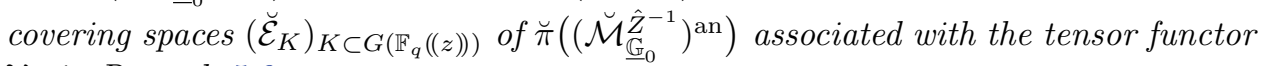
$\underline{\mathcal{V}}_{b}$ in Remark 5.9.

Remark 8.2. (a) Note that if the analogue of Wintenberger's theorem (5.6) is established, the union of connected components in (a) is simply $\breve{\mathcal{H}}_{G, \hat{Z}, b}^{n a}$. In particular, this would imply that $\breve{\mathcal{M}}^{K} \neq \emptyset$ if and only if $[b] \in B\left(G, \hat{Z}_{E}\right)$. This is the analogue of $[79$, Conjecture 4.21].

(b) By Theorem 8.1(c), the tower $\left(\breve{\mathcal{M}}^{K}\right)_{K \subset G\left(\mathbb{F}_{q}((z))\right)}$ only depends on the triple $\left(G_{\mathbb{F}_{q}((z))},[b], \hat{Z}_{E}\right)$, where $[b]$ is the $\sigma$-conjugacy class of $b$ under $L G(\mathbb{F})$. Indeed, the tower $\left(\breve{\mathcal{E}}_{K}\right)_{K \subset G\left(\mathbb{F}_{q}((z))\right)}$ only depends on this triple; see Definition 4.16 and Remark 5.9. In the arithmetic situation, the analogues of the tower $\left(\breve{\mathcal{M}}^{K}\right)_{K \subset G\left(\mathbb{F}_{q}((z))\right)}$ are called local Shimura varieties; see $[79, \S 5.2]$ and $[90, \S 24]$. For the same reason they also only depend on the group scheme over $\mathbb{Q}_{p}$, the bounding cocharacter $\mu$ and the $\sigma$-conjugacy class $[b]$; see [90, Proposition 23.2.1 and thereafter].

To prove Theorem 8.1, we will take a Tannakian approach and make use of the following proposition.

Proposition 8.3. Let $G$ be a faithfully flat affine group scheme over a Dedekind domain $A$ and let $B$ be an $A$-algebra. Let $\operatorname{Rep}_{A} G$ be the Tannakian category of representations of $G$ on finite projective $A$-modules and let $\omega^{\circ}: \operatorname{Rep}_{A} G \rightarrow F M o d_{A}$ be the forgetful fibre functor. Let $\omega: \operatorname{Rep}_{A} G \rightarrow F_{B o d}$ be a tensor functor to the category FMod ${ }_{B}$ of finite projective $B$-modules, which sends morphisms in $\operatorname{Rep}_{A} G$ that are epimorphisms on the underlying $A$-modules to epimorphisms in FMod $_{B}$. Then $\operatorname{Isom}^{\otimes}\left(\omega^{\circ} \otimes_{A} B, \omega\right)$ is representable by a $G$-torsor over $B$ (for the fpqc topology).

Proof. Let $B G=[\operatorname{Spec} A / G]$ be the classifying stack of $G$ that parametrises $G$-torsors over $A$-schemes. By [32, Tags 0443 and 06WS], the category of linear representations of $G$ on (arbitrary) $A$-modules is tensor equivalent to the category $\operatorname{Mod}_{B G}$ of quasi-coherent sheaves on (the big fppf-site of) $B G$; see [32, Tags 06NT and 03DL]. This equivalence is given by the functor that sends a quasi-coherent sheaf $\mathcal{F}$ on $B G$ to its pullback under the morphism $p_{0}$ : Spec $A \rightarrow B G$. The quasi-inverse functor sends a representation of $G$ on an $A$-module $F$ to its faithfully flat descent on $B G$. By faithfully flat descent $\left[44, \mathrm{IV}_{2}\right.$, Proposition 2.5.2], $\mathcal{F}$ is finite locally free if and only if the representation is a finite locally 
free $A$-module. In particular, the category $\operatorname{Rep}_{A} G$ is tensor equivalent to the category FMod $_{B G}$ of finite locally free sheaves on $B G$.

If we write $G=\operatorname{Spec} \Gamma$, the multiplication of $G$ makes $\Gamma$ into a comodule, which is the filtered union of its finitely generated subcomodules by [91, $\S 1.5$, Corollaire]. Because $\Gamma$ is a flat $A$-module and $A$ is Dedekind, these finitely generated subcomodules are torsion free and hence finite projective and dualisable. The Hopf algebroid $(A, \Gamma)$ is therefore an Adams Hopf algebroid and the stack $B G$ is an Adams stack; see [85, §1.3]. Due to our assumption, the tensor functor $\omega$ defines a tensor functor from $\mathrm{FMod}_{B G}$ to $\operatorname{Mod}_{B}$ that sends locally split epimorphisms $\varphi$ to epimorphisms. Indeed, if $U$ is a scheme and $u: U \rightarrow B G$ is an $f p q c$ covering over which $u^{*} \varphi$ splits, then $u^{*} \varphi$ is an epimorphism of quasi-coherent sheaves on $U$. Because $U \times_{B G} \operatorname{Spec} A \rightarrow \operatorname{Spec} A$ is an fpqc covering, fpqc descent [44, $\mathrm{IV}_{2}$, Proposition 2.2.7] shows that $p_{0}^{*} \varphi$ is an epimorphism on the underlying $A$-modules, and our assumption applies. Thus, by [86, Corollary 3.4.3] and [85, Theorems 1.3.2 and 1.2.1], the functor $\omega$ corresponds to a morphism $f: \operatorname{Spec} B \rightarrow B G$ and an isomorphism of tensor functors $\alpha: f^{*} \stackrel{\sim}{\longrightarrow} \omega \circ p_{0}^{*}$.

By definition of $B G$, this morphism corresponds to a $G$-torsor $p: X \rightarrow \operatorname{Spec} B$, which is obtained as the base change

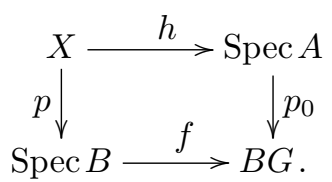

We will show that $X$ represents Isom ${ }^{\otimes}\left(\omega^{\circ} \otimes_{A} B, \omega\right)$. Because $p_{0}^{*}$ is an equivalence between $\operatorname{FMod}_{B G}$ and $\operatorname{Rep}_{A} G$, the tensor functor $p^{*} \alpha: h^{*} \circ p_{0}^{*}=p^{*} \circ f^{*} \stackrel{\sim}{\longrightarrow} p^{*} \circ \omega \circ p_{0}^{*}$ induces a tensor isomorphism $p^{*} \circ\left(\omega^{\circ} \otimes_{A} B\right)=h^{*} \stackrel{\sim}{\longrightarrow} p^{*} \circ \omega$ over $X$ and hence a morphism $X \rightarrow$ Isom $^{\otimes}\left(\omega^{\circ} \otimes_{A} B, \omega\right)$. To prove that the latter is an isomorphism, let $s: S=\operatorname{Spec} R \rightarrow$ Spec $B$ and let $\beta \in \operatorname{Isom}^{\otimes}\left(\omega^{\circ} \otimes_{A} B, \omega\right)(S)$, which we view as a tensor isomorphism $\beta: s^{*} \circ$ $\left(\omega^{\circ} \otimes_{A} B\right) \circ p_{0}^{*} \stackrel{\sim}{\longrightarrow} s^{*} \circ \omega \circ p_{0}^{*}$. Let $r: \operatorname{Spec} B \rightarrow \operatorname{Spec} A$ be the structure morphism. The two morphisms $f \circ s$ and $p_{0} \circ r \circ s$ from $S$ to $B G$ induce the pullback functors $s^{*} \circ f^{*}$ and $s^{*} \circ r^{*} \circ p_{0}^{*}=s^{*} \circ\left(\omega^{\circ} \otimes_{A} B\right) \circ p_{0}^{*}$, which are isomorphic by

$$
\beta^{-1} \circ s^{*} \alpha: s^{*} \circ f^{*} \stackrel{\sim}{\longrightarrow} s^{*} \circ \omega \circ p_{0}^{*} \stackrel{\sim}{\longrightarrow} s^{*} \circ\left(\omega^{\circ} \otimes_{A} B\right) \circ p_{0}^{*} .
$$

Again by [86, Corollary 3.4.3] and [85, Theorems 1.3.2 and 1.2.1], the latter isomorphism corresponds to an isomorphism $\eta$ between the morphisms $f \circ s$ and $p_{0} \circ r \circ s$ from $S$ to $B G$. The data $(s, r \circ s, \eta)$ define a morphism $S \rightarrow X$ and this proves that $X \stackrel{\sim}{\longrightarrow} \operatorname{Isom}^{\otimes}\left(\omega^{\circ} \otimes_{A}\right.$ $B, \omega)$ as desired.

We also need the following easy lemma.

Lemma 8.4. Let $\Omega$ be an algebraically closed field, which is complete with respect to an absolute value $||:. \Omega \rightarrow \mathbb{R}_{\geq 0}$, and let $\mathcal{O}_{\Omega}$ be its valuation ring. Let $\mathfrak{m}_{\Omega} \subset \mathcal{O}_{\Omega}$ be the maximal ideal and $\kappa_{\Omega}=\mathcal{O}_{\Omega} / \mathfrak{m}_{\Omega}$.

(a) If $\mathfrak{p} \subset \mathcal{O}_{\Omega} \llbracket z \rrbracket$ is a prime ideal then one of the following assertions holds:

(i) $\mathfrak{p}$ is contained in $\mathfrak{p}_{0}:=\left\{\sum_{i=0}^{\infty} b_{i} z^{i}: b_{i} \in \mathfrak{m}_{\Omega}\right\}=\operatorname{ker}\left(\mathcal{O}_{\Omega} \llbracket z \rrbracket \rightarrow \kappa_{\Omega} \llbracket z \rrbracket\right)$, 
(ii) $\mathfrak{p}=(z-\alpha)$ for an $\alpha \in \mathfrak{m}_{\Omega}$ or

(iii) $\mathfrak{p}=(z)+\mathfrak{m}_{\Omega}$ is the maximal ideal of $\mathcal{O}_{\Omega} \llbracket z \rrbracket$.

(b) The maximal ideals $\mathfrak{m}$ of the ring $\mathcal{O}_{\Omega}((z)):=\mathcal{O}_{\Omega} \llbracket z \rrbracket\left[\frac{1}{z}\right]$ are all of the following form: $\mathfrak{m}=(z-\alpha)$ for $\alpha \in \mathfrak{m}_{\Omega} \backslash\{0\}$ with $\mathcal{O}_{\Omega}((z)) / \mathfrak{m}=\Omega$ or $\mathfrak{m}=\mathfrak{m}_{0}:=\left\{\sum_{i} b_{i} z^{i}: b_{i} \in\right.$ $\left.\mathfrak{m}_{\Omega}\right\}=\operatorname{ker}\left(\mathcal{O}_{\Omega}((z)) \rightarrow \kappa_{\Omega}((z))\right)$ with $\mathcal{O}_{\Omega}((z)) / \mathfrak{m}=\kappa_{\Omega}((z))$.

Proof. (a) If (i) fails - that is, if $\mathfrak{p} \not \subset \mathfrak{p}_{0}$ - then there is an element $f=\sum_{i=0}^{\infty} b_{i} z^{i} \in \mathfrak{p}$ with $b_{n} \in \mathcal{O}_{\Omega}^{\times}$for some $n$. We may assume that $b_{i} \in \mathfrak{m}_{\Omega}$ for all $i<n$, and hence the image $\bar{f}$ of $f$ in $\kappa_{\Omega} \llbracket z \rrbracket$ has $\operatorname{ord}_{z}(\bar{f})=n$, where $\operatorname{ord}_{z}$ is the valuation of the discrete valuation ring $\kappa_{\Omega} \llbracket z \rrbracket$. If $n=0$, then $f$ would be a unit, which is not the case. So $\left|b_{0}\right|<\left|b_{n}\right|=1$ and the Newton polygon of $f$ has a negative slope. By [74, Proposition 2] there is an element $\alpha \in \mathfrak{m}_{\Omega}$ and a power series $g=\sum_{i=0}^{\infty} c_{i} z^{i} \in \Omega \llbracket z \rrbracket$ with $f=(z-\alpha) \cdot g$; that is, $b_{i}=c_{i-1}-\alpha c_{i}$, such that the region of convergence of $f$ is contained in the one of $g$. In particular, $g$ converges for all $z$ in $\mathfrak{m}_{\Omega}$.

We claim that $g \in \mathcal{O}_{\Omega} \llbracket z \rrbracket$; that is, $\left|c_{i}\right| \leq 1$ for all $i$. Assume contrarily that there exists an $m>0$ with $\left|c_{m-1}\right|>1 \geq\left|b_{m}\right|$. Then $\alpha c_{m}=c_{m-1}-b_{m}$ implies $\left|c_{m}\right|=\left|\alpha^{-1} c_{m-1}\right|>\left|c_{m-1}\right|$ and by induction $\left|c_{i}\right|=|\alpha|^{m-1-i}\left|c_{m-1}\right|$ for all $i \geq m-1$. This implies that $g$ does not converge at $z=\alpha$. So we obtain a contradiction and the claim is proved.

For the images in $\kappa_{\Omega} \llbracket z \rrbracket$ we obtain $\bar{f}=z \cdot \bar{g}$ and $\operatorname{ord}_{z}(\bar{g})=\operatorname{ord}_{z}(\bar{f})-1$. Continuing in this way, we find that $f=\left(z-\alpha_{1}\right) \cdot \ldots \cdot\left(z-\alpha_{n}\right) \cdot h$ for $\alpha_{i} \in \mathfrak{m}_{\Omega}$ and a unit $h \in \mathcal{O}_{\Omega} \llbracket z \rrbracket^{\times}$. Because $\mathfrak{p}$ is prime, it contains $z-\alpha_{i}$ for some $i$. Under the isomorphism $\mathcal{O}_{\Omega} \llbracket z \rrbracket /\left(z-\alpha_{i}\right) \stackrel{\sim}{\longrightarrow} \mathcal{O}_{\Omega}$, $z \mapsto \alpha_{i}$, the quotient $\mathfrak{p} /\left(z-\alpha_{i}\right)$ is a prime ideal in $\mathcal{O}_{\Omega}$. If $\mathfrak{p} /\left(z-\alpha_{i}\right)=(0)$, then $\mathfrak{p}=\left(z-\alpha_{i}\right)$ and we are in case (ii). If $\mathfrak{p} /\left(z-\alpha_{i}\right)=\mathfrak{m}_{\Omega}$, then $\mathfrak{p}=\left(z-\alpha_{i}\right)+\mathfrak{m}_{\Omega}=(z)+\mathfrak{m}_{\Omega}$ and we are in case (iii).

(b) follows from (a) via the identification of the prime ideals in $\mathcal{O}_{\Omega}((z))$ with the prime ideals in $\mathcal{O}_{\Omega} \llbracket z \rrbracket$ not containing $z$.

Proof of Theorem 8.1. (a) Because the proof is quite long and involved, let us give a summary first. By [31, Theorem 2.9], the subset of points $\bar{\gamma}$ admitting an $\mathbb{F}_{q}((z))$ rational isomorphism $\beta: \omega^{\circ} \stackrel{\sim}{\longrightarrow} \omega_{b, \bar{\gamma}}$ between the tensor functors $\omega^{\circ}$ and $\omega_{b, \bar{\gamma}}$ is a union of connected components. Thus, the task is to prove that it agrees with the image of the period morphism.

We start with a point $\bar{\gamma} \in \breve{\mathcal{H}}_{G, \hat{Z}, b}^{a}(\Omega)$ with values in an algebraically closed, complete field $\Omega$ and a tensor isomorphism $\beta$. In Step 1 we construct from these data a tensor functor $\underline{\mathcal{T}}_{b}: \operatorname{Rep}_{\mathbb{F}_{q} \llbracket z \rrbracket} G \rightarrow \mathbb{F}_{q} \llbracket z \rrbracket-\underline{\operatorname{Loc}}_{\breve{\mathcal{E}}_{K_{0}}}$ over an étale covering space $\breve{\mathcal{E}}_{K_{0}}$ of $\breve{\mathcal{H}}_{G, \hat{z}, b}^{a}$. By the admissibility of $\bar{\gamma}$, in Step 2 this tensor functor will induce a tensor functor $M: \operatorname{Rep}_{\mathbb{F}_{q} \llbracket z \rrbracket} G \rightarrow \operatorname{FMod}_{\mathcal{O}_{\Omega} \llbracket z \rrbracket}, V \mapsto M_{V}$, which gives the underlying module $M_{V}$ of a local shtuka $\underline{M}_{V}$. Unfortunately, it is not clear that the functor $V \mapsto M_{V}$ is exact without using the hypothesis that $G$ is parahoric. This might even be false for groups $G$ with nonreductive generic fibre, as Example 8.6 shows. Nevertheless, we show in Step 3 that $V \mapsto M_{V} \otimes_{\mathcal{O}_{\Omega} \llbracket z \rrbracket} \mathcal{O}_{\Omega}((z))$ is exact and corresponds to a $G$-torsor $\mathcal{G}$ over $\mathcal{O}_{\Omega}((z))$. From [3, Proposition 11.5], we obtain that $\mathcal{G} \cong G \otimes_{\mathbb{F}_{q} \llbracket z \rrbracket} \mathcal{O}_{\Omega}((z))$ is trivial. This allows us in Step 4 to apply Lemma 7.9 to produce a local $G$-shtuka $\left(\underline{\mathcal{G}}^{\prime \prime}, \bar{\Delta}^{\prime \prime}\right) \in \breve{\mathcal{M}}_{\underline{\mathbb{G}}_{0}}^{\hat{Z}^{-1}}\left(\mathcal{O}_{\Omega}\right)$ with $\breve{\pi}\left(\underline{\mathcal{G}}^{\prime \prime}, \bar{\Delta}^{\prime \prime}\right)=\bar{\gamma}$. 
1. Let $\gamma \in \breve{\mathcal{H}}_{G, \hat{Z}, b}^{a}$ and let $\bar{\gamma}$ be a geometric point lying above $\gamma$ with algebraically closed, complete residue field $\Omega$. We consider the tensor functor $\underline{\mathcal{V}}_{b}: \operatorname{Rep}_{\mathbb{F}_{q}((z))} G \rightarrow$ $\mathbb{F}_{q}((z))$-Loc $_{\breve{\mathcal{H}}_{G, \hat{z}, b}^{a}}$ from Theorem 5.7 and the two fibre functors $\omega_{b, \bar{\gamma}}:=$ forget $\circ \omega_{\bar{\gamma}} \circ \underline{\mathcal{V}}_{b}$ and $\omega^{\circ}: \operatorname{Rep}_{\mathbb{F}_{q}((z))} G \rightarrow \operatorname{FMod}_{\mathbb{F}_{q}((z))}$ from Remark 5.8 and assume that there is an $\mathbb{F}_{q}((z))$ rational isomorphism

$$
\beta: \omega^{\circ} \stackrel{\sim}{\longrightarrow} \omega_{b, \bar{\gamma}}
$$

of tensor functors that we fix. It induces an isomorphism of group schemes $\widetilde{G}:=\operatorname{Aut}^{\otimes}\left(\omega_{b, \bar{\gamma}}\right) \stackrel{\sim}{\longrightarrow} \operatorname{Aut}^{\otimes}\left(\omega_{0}\right)=G_{\mathbb{F}_{q}((z))}$ over $\mathbb{F}_{q}((z))$. Consider the compact open subgroup $K_{0}:=G\left(\mathbb{F}_{q} \llbracket z \rrbracket\right) \subset G\left(\mathbb{F}_{q}((z))\right)$ and the étale covering space $\breve{\mathcal{E}}_{K_{0}}$ of $\breve{\mathcal{H}}_{G, \hat{Z}, b}^{a}$ from Remark 5.5 associated with the local system $\underline{\mathcal{V}}_{b}$ on $\breve{\mathcal{H}}_{G, \hat{z}, b}^{a}$. The point $\beta K_{0} \in$ $\operatorname{Isom}^{\otimes}\left(\omega^{\circ}, \omega_{b, \bar{\gamma}}\right)\left(\mathbb{F}_{q}((z))\right) / K_{0}=F_{\bar{\gamma}}^{\text {ét }}\left(\breve{\mathcal{E}}_{K_{0}}\right)$ corresponds to a lift of the base point $\bar{\gamma}$ to a geometric base point $\bar{\gamma}$ of $\breve{\mathcal{E}}_{K_{0}}$ such that the morphism $\pi_{1}^{\text {et }}\left(\breve{\mathcal{E}}_{K_{0}}, \bar{\gamma}\right) \rightarrow \pi_{1}^{\text {ét }}\left(\breve{\mathcal{H}}_{G, \hat{z}, b}^{a}, \bar{\gamma}\right) \rightarrow$ $G\left(\mathbb{F}_{q}((z))\right)$ from (5.7) factors through $K_{0}$. By Corollary 5.4 the induced morphism $\pi_{1}^{\text {ét }}\left(\breve{\mathcal{E}}_{K_{0}}, \bar{\gamma}\right) \rightarrow K_{0} \quad$ yields a tensor functor $\underline{\mathcal{T}}_{b}: \operatorname{Rep}_{\mathbb{F}_{q} \llbracket z \rrbracket} G \rightarrow \mathbb{F}_{q} \llbracket z \rrbracket-\underline{\operatorname{Loc}}_{\breve{\mathcal{E}}_{K_{0}}}$ and an isomorphism $\omega^{\circ} \stackrel{\sim}{\longrightarrow}$ forget $\circ \omega_{\bar{\gamma}} \circ \underline{\mathcal{T}}_{b}$, which equals the restriction of $\beta$ by construction. In particular, for each $V \in \operatorname{Rep}_{\mathbb{F}_{q} \llbracket z \rrbracket} G$, the fibre $\underline{\mathcal{T}}_{b}(V)_{\bar{\gamma}}$ is a free $\mathbb{F}_{q} \llbracket z \rrbracket$-module of rank equal to the rank of $V$.

2. Let $\left(\rho_{V}, V\right) \in \operatorname{Rep}_{\mathbb{F}_{q} \llbracket z \rrbracket} G$. Then the $z$-isocrystal with Hodge-Pink structure

$$
\underline{D}_{b, \bar{\gamma}}(V)=\left(V \otimes_{\mathbb{F}_{q} \llbracket z \rrbracket} \mathbb{F}((z)), \rho_{V}\left(\sigma^{*} b\right) \sigma^{*}, \mathfrak{q}_{D}(V)\right)
$$

over $\Omega$ is admissible and comes from a pair $\left(\underline{M}_{V}, \delta_{V}\right)$ where $\underline{M}_{V}=\left(M_{V}, \tau_{M_{V}}\right)$ is a local shtuka over $\mathcal{O}_{\Omega}$ with $M_{V} \cong \mathcal{O}_{\Omega} \llbracket z \rrbracket^{r}$ and $\tau_{M_{V}}: \sigma^{*} M_{V}\left[\frac{1}{z-\zeta}\right] \stackrel{\sim}{\sim} M_{V}\left[\frac{1}{z-\zeta}\right]$ and with the notation (6.1),

$$
\left.\left.\delta_{V}: \underline{M}_{V} \otimes_{\mathcal{O}_{\Omega} \llbracket z \rrbracket} \mathcal{O}_{\Omega} \llbracket z, z^{-1}\right\}\left[\frac{1}{t_{-}}\right] \stackrel{\sim}{\longrightarrow}\left(V \otimes_{\mathbb{F}_{q} \llbracket z \rrbracket} \mathcal{O}_{\Omega} \llbracket z, z^{-1}\right\}\left[\frac{1}{t_{-}}\right], \rho_{V}(b) \sigma^{*}\right)
$$

is an isomorphism satisfying $\delta_{V} \circ \tau_{M_{V}}=\rho_{V}(b) \circ \sigma^{*} \delta_{V}$, such that

$$
\mathfrak{q}_{D}(V):=\rho_{V}(\bar{\gamma}) \cdot V \otimes_{\mathbb{F}_{q} \llbracket z \rrbracket} \Omega \llbracket z-\zeta \rrbracket=\sigma^{*} \delta_{V} \circ \tau_{M_{V}}^{-1}\left(M_{V} \otimes_{\mathcal{O}_{\Omega} \llbracket z \rrbracket} \Omega \llbracket z-\zeta \rrbracket\right)
$$

and so

$$
\tau_{M_{V}} \circ \sigma^{*} \delta_{V}^{-1} \circ \rho_{V}(\bar{\gamma}): V \otimes_{\mathbb{F}_{q} \llbracket z \rrbracket} \Omega \llbracket z-\zeta \rrbracket \stackrel{\sim}{\longrightarrow} M_{V} \otimes_{\mathcal{O}_{\Omega} \llbracket z \rrbracket} \Omega \llbracket z-\zeta \rrbracket .
$$

This follows from [50, Proposition 2.4.9] with $X_{L}=\operatorname{Sp} \Omega$ and $X=\operatorname{Spf} \mathcal{O}_{\Omega}$, where our pair $\left(\underline{M}_{V}, \delta_{V}\right)$ is the rigidified local shtuka of [50, Proposition 2.4.9] We may apply this proposition by taking $\mathcal{Q}^{\prime}:=\underline{\mathcal{T}}_{b}(V)_{\bar{\gamma}} \otimes_{\mathbb{F}_{q} \llbracket z \rrbracket} \Omega\left\langle\frac{z}{\zeta^{s}}\right\rangle$ inside $\mathcal{Q}:=\underline{\mathcal{F}}_{b, \bar{\gamma}}(V)=\mathcal{Q}^{\prime} \otimes_{\Omega\left\langle\frac{z}{\zeta^{s}}\right\rangle} \Omega\left\langle\frac{z}{\zeta^{s}}, z^{-1}\right\}$ for an $s$ with $1>s>\frac{1}{q}$; see Remark 5.6. Note that our notation here of the underlying $z$ isocrystal deviates from [50]. Namely, our $\underline{D}_{b}(V)=\left(V \otimes_{\mathbb{F}_{q} \llbracket z \rrbracket} \mathbb{F}((z)), \rho_{V}\left(\sigma^{*} b\right) \sigma^{*}\right)$ was called $\sigma^{*}\left(D, F_{D}\right)$ in [50, Proposition 2.4.9]. So the $\left(D, F_{D}\right)$ from [50, Proposition 2.4.9] is equal to our $\left(V \otimes_{\mathbb{F}_{q} \llbracket z \rrbracket} \mathbb{F}((z)), \rho_{V}(b) \sigma^{*}\right)$. The latter equals the $z$-isocrystal associated with the local GL(V)-shtuka $\rho_{V, * \mathbb{G}_{0}}$ over $\mathbb{F}$ that was used in (the proof of) Lemma 6.1. 
As can be seen from the proof of [50, Proposition 2.4.9], there is an isomorphism $\varepsilon_{V}: \underline{M}_{V} \otimes_{\mathcal{O}_{\Omega} \llbracket z \rrbracket} \Omega\left\langle\frac{z}{\zeta^{s}}\right\rangle \stackrel{\sim}{\longrightarrow} \mathcal{Q}^{\prime}=\underline{\mathcal{T}}_{b}(V)_{\bar{\gamma}} \otimes_{\mathbb{F}_{q} \llbracket z \rrbracket} \Omega\left\langle\frac{z}{\zeta^{s}}\right\rangle$ yielding $\check{T}_{z} \underline{M}_{V} \cong\left(\mathcal{Q}^{\prime}\right)^{\tau}=\underline{\mathcal{T}}_{b}(V)_{\bar{\gamma}}$ Alternatively, $\left[50\right.$, Proposition 2.4.4] provides an isomorphism $\varepsilon_{V}: \underline{M}_{V} \otimes_{\mathcal{O}_{\Omega} \llbracket z \rrbracket}$ $\Omega\left\langle\frac{z}{\zeta^{s}}, z^{-1}\right\} \stackrel{\sim}{\longrightarrow} \underline{\mathcal{F}}_{b, \bar{\gamma}}(V)$ yielding $\check{T}_{z} \underline{M}_{V} \otimes_{\mathbb{F}_{q} \llbracket z \rrbracket} \mathbb{F}_{q}((z)) \cong\left(\underline{\mathcal{F}}_{b, \bar{\gamma}}(V)\right)^{\tau}=\underline{\mathcal{T}}_{b}(V)_{\bar{\gamma}} \otimes_{\mathbb{F}_{q} \llbracket z \rrbracket}$ $\mathbb{F}_{q}((z))=\underline{\mathcal{V}}_{b}(V)_{\bar{\gamma}}$. Now the existence of a uniquely determined pair $\left(\underline{M}_{V}, \delta_{V}\right)$ with $\varepsilon_{V}\left(\check{T}_{z} \underline{M}_{V}\right)=\underline{\mathcal{T}}_{b}(V)_{\bar{\gamma}}$ follows from Proposition 7.8.

We claim that the underlying free $\mathcal{O}_{\Omega} \llbracket z \rrbracket$-module $M_{V}$ of the local shtuka $\underline{M}_{V}$ defines a tensor functor $M: \operatorname{Rep}_{\mathbb{F}_{q} \llbracket z \rrbracket} G \rightarrow \operatorname{FMod}_{\mathcal{O}_{\Omega} \llbracket z \rrbracket}, V \mapsto M_{V}$. We use the isomorphisms

$$
\begin{gathered}
\left.\left.\sigma^{*} \delta_{V} \circ \tau_{M_{V}}^{-1}: M_{V} \otimes_{\mathcal{O}_{\Omega} \llbracket z \rrbracket} \mathcal{O}_{\Omega} \llbracket z, z^{-1}\right\}\left[\frac{1}{t_{-}}\right] \stackrel{\sim}{\sim} V \otimes_{\mathbb{F}_{q} \llbracket z \rrbracket} \mathcal{O}_{\Omega} \llbracket z, z^{-1}\right\}\left[\frac{1}{t_{-}}\right] \quad \text { and } \\
\left(\beta_{V} \otimes \operatorname{id}_{\Omega\left\langle\frac{z}{\zeta^{s}}\right\rangle}\right)^{-1} \circ \varepsilon_{V}: M_{V} \otimes_{\mathcal{O}_{\Omega} \llbracket z \rrbracket} \Omega\left\langle\frac{z}{\zeta^{s}}\right\rangle \stackrel{\sim}{\sim} \underline{\mathcal{T}}_{b}(V)_{\bar{\gamma}} \otimes_{\mathbb{F}_{q} \llbracket z \rrbracket} \Omega\left\langle\frac{z}{\zeta^{s}}\right\rangle \stackrel{\sim}{\longrightarrow} V \otimes_{\mathbb{F}_{q} \llbracket z \rrbracket} \Omega\left\langle\frac{z}{\zeta^{s}}\right\rangle,
\end{gathered}
$$

where $\beta_{V}: V=\omega(V)^{\circ} \stackrel{\sim}{\longrightarrow} \omega_{b, \bar{\gamma}}(V)$ is induced from the isomorphism $\beta$ from (8.1). Consider the identification

$h_{V}: \underline{\mathcal{T}}_{b}(V)_{\bar{\gamma}} \otimes_{\mathbb{F}_{q} \llbracket z \rrbracket} \Omega\left\langle\frac{z}{\zeta^{s}}, z^{-1}\right\}\left[\frac{1}{t_{-}}\right]=\mathcal{F}_{b, \bar{\gamma}}(V)\left[\frac{1}{t_{-}}\right]=\mathcal{E}_{b, \bar{\gamma}}(V)\left[\frac{1}{t_{-}}\right]:=V \otimes_{\mathbb{F}_{q} \llbracket z \rrbracket} \Omega\left\langle\frac{z}{\zeta^{s}}, z^{-1}\right\}\left[\frac{1}{t_{-}}\right]$.

Then the isomorphisms $\sigma^{*} \delta_{V} \circ \tau_{M_{V}}^{-1}$ and $\left(\beta_{V} \otimes \operatorname{id}_{\Omega\left\langle\frac{z}{\zeta^{s}}\right\rangle}\right)^{-1} \circ \varepsilon_{V}$ satisfy

$$
\begin{aligned}
& h_{V} \circ\left(\beta_{V} \otimes \operatorname{id}_{\Omega\left\langle\frac{z}{\zeta^{s}}, z^{-1}\right\}\left[\frac{1}{t_{-}}\right]}\right) \circ\left(\left(\beta_{V} \otimes \operatorname{id}_{\Omega\left\langle\frac{z}{\zeta^{s}}\right\rangle}\right)^{-1} \circ \varepsilon_{V}\right) \otimes \operatorname{id}_{\Omega\left\langle\frac{z}{\zeta^{s}}, z^{-1}\right\}\left[\frac{1}{t_{-}}\right]} \\
& \left.\quad=\left(\sigma^{*} \delta_{V} \circ \tau_{M_{V}}^{-1}\right) \otimes \operatorname{id}_{\Omega\left\langle\frac{z}{\zeta^{s}}\right.}, z^{-1}\right\}\left[\frac{1}{t_{-}}\right] \\
& : M_{V} \otimes_{\mathcal{O}_{\Omega} \llbracket z \rrbracket} \Omega\left\langle\frac{z}{\zeta^{s}}, z^{-1}\right\}\left[\frac{1}{t_{-}}\right] \stackrel{\sim}{\longrightarrow} \mathcal{E}_{b, \bar{\gamma}}(V)\left[\frac{1}{t_{-}}\right]
\end{aligned}
$$

and $M_{V}$ is obtained from the intersection

$$
\left.\sigma^{*} \delta_{V} \circ \tau_{M_{V}}^{-1}\left(M_{V}\right)=V \otimes_{\mathbb{F}_{q} \llbracket z \rrbracket} \mathcal{O}_{\Omega} \llbracket z, z^{-1}\right\}\left[\frac{1}{t_{-}}\right] \cap h_{V} \beta_{V}\left(V \otimes_{\mathbb{F}_{q} \llbracket z \rrbracket} \Omega\left\langle\frac{z}{\zeta^{s}}\right\rangle\right)
$$

inside $V \otimes_{\mathbb{F}_{q} \llbracket z \rrbracket} \Omega\left\langle\frac{z}{\zeta^{s}}, z^{-1}\right\}\left[\frac{1}{t_{-}}\right]$.

To prove compatibility with morphisms $f: V \rightarrow V^{\prime}$ in $\operatorname{Rep}_{\mathbb{F}_{q} \llbracket z \rrbracket} G$, we consider

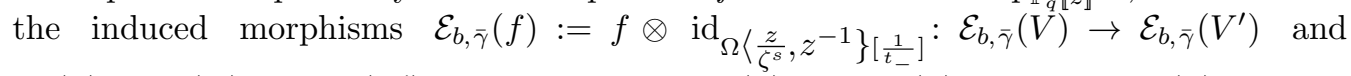
$\underline{\mathcal{T}}_{b}(f)_{\bar{\gamma}}: \underline{\mathcal{I}}_{b}(V)_{\bar{\gamma}} \rightarrow \underline{\mathcal{T}}_{b}\left(V^{\prime}\right)_{\bar{\gamma}}$. Because $h_{V^{\prime}} \circ \underline{\mathcal{T}}_{b}(f)_{\bar{\gamma}}=\mathcal{E}_{b, \bar{\gamma}}(f) \circ h_{V}$ and $\underline{\mathcal{T}}_{b}(f)_{\bar{\gamma}} \circ \beta_{V}=$ $\beta_{V^{\prime}} \circ f$, the morphism $f$ induces via (8.6) a morphism $M_{f}: M_{V} \rightarrow M_{V^{\prime}}$.

Finally, if $V, V^{\prime} \in \operatorname{Rep}_{\mathbb{F}_{q} \llbracket z \rrbracket} G$ then $\left(\underline{M}_{V}, \delta_{V}\right) \otimes\left(\underline{M}_{V^{\prime}}, \delta_{V^{\prime}}\right)$ has $\underline{D}_{b, \bar{\gamma}}(V) \otimes \underline{D}_{b, \bar{\gamma}}\left(V^{\prime}\right)=$ $\underline{D}_{b, \bar{\gamma}}\left(V \otimes V^{\prime}\right)$ as $z$-isocrystal with Hodge-Pink structure and $\check{T}_{z} \underline{M}_{V} \otimes \check{T}_{z} \underline{M}_{V^{\prime}} \cong \underline{\mathcal{T}}_{b}(V)_{\bar{\gamma}} \otimes$ $\underline{\mathcal{T}}_{b}\left(V^{\prime}\right)_{\bar{\gamma}}=\underline{\mathcal{T}}_{b}\left(V \otimes V^{\prime}\right)_{\bar{\gamma}}$ as Tate-module. This implies that $\left(\underline{M}_{V \otimes V^{\prime}}, \delta_{V \otimes V^{\prime}}\right)=\left(\underline{M}_{V}, \delta_{V}\right) \otimes$ $\left(\underline{M}_{V^{\prime}}, \delta_{V^{\prime}}\right)$ and so $M$ is indeed a tensor functor.

Unfortunately, it is not a priori clear that this tensor functor comes from a $G$-torsor over $\mathcal{O}_{\Omega} \llbracket z \rrbracket$. Namely, a necessary (and by Proposition 8.3 also sufficient) condition is that for every sequence $0 \rightarrow V^{\prime} \rightarrow V \rightarrow V^{\prime \prime} \rightarrow 0$ in $\operatorname{Rep}_{\mathbb{F}_{q} \llbracket z \rrbracket} G$, which is exact on the underlying free $\mathbb{F}_{q} \llbracket z \rrbracket$-modules; also, the sequence $0 \rightarrow M_{V^{\prime}} \rightarrow M_{V} \rightarrow M_{V^{\prime \prime}} \rightarrow 0$ of free $\mathcal{O}_{\Omega} \llbracket z \rrbracket$-modules is exact. This is not obvious without using the hypothesis that $G$ is parahoric and may even be false if the generic fibre of $G$ is not reductive as Example 8.6 below shows. 
3. We first claim that the sequence of $\mathcal{O}_{\Omega} \llbracket z \rrbracket$-modules $0 \rightarrow M_{V^{\prime}} \rightarrow M_{V} \rightarrow M_{V^{\prime \prime}} \rightarrow 0$ becomes exact after tensoring with $\mathcal{O}_{\Omega}((z)):=\mathcal{O}_{\Omega} \llbracket z \rrbracket\left[\frac{1}{z}\right]$. Namely, because the cokernel $C$ of $M_{V}\left[\frac{1}{z}\right] \rightarrow M_{V^{\prime \prime}}\left[\frac{1}{z}\right]$ is finitely generated, it suffices by Nakayama's lemma to prove that $C / \mathfrak{m} C=(0)$ for every maximal ideal $\mathfrak{m} \subset \mathcal{O}_{\Omega}((z))$. By Lemma 8.4(b) there are two cases, namely, $\mathfrak{m}=\mathfrak{m}_{0}=\operatorname{ker}\left(\mathcal{O}_{\Omega}((z)) \rightarrow \kappa_{\Omega}((z))\right)$ and $\mathfrak{m}=(z-\alpha)$ for $\alpha \in \mathfrak{m}_{\Omega} \backslash\{0\}$. In the first case, the morphism $\mathcal{O}_{\Omega} \llbracket z \rrbracket \rightarrow \mathcal{O}_{\Omega}((z)) / \mathfrak{m}_{0}=\kappa_{\Omega}((z))$ factors through $\left.\mathcal{O}_{\Omega} \llbracket z, z^{-1}\right\}\left[\frac{1}{t_{-}}\right]$, because $t_{-} \equiv 1\left(\bmod \mathfrak{m}_{0}\right)$, and hence $C / \mathfrak{m}_{0} C \cong \operatorname{coker}\left(V \rightarrow V^{\prime \prime}\right) \otimes_{\mathbb{F}_{q} \llbracket z \rrbracket} \kappa_{\Omega}((z))=(0)$ by using the isomorphism (8.4). In the second case, if $|\alpha|>|\zeta|^{s}$, the morphism $\mathcal{O}_{\Omega} \llbracket z \rrbracket \rightarrow \mathcal{O}_{\Omega}((z)) / \mathfrak{m}=$ $\Omega, z \mapsto \alpha$ likewise factors through $\left.\mathcal{O}_{\Omega} \llbracket z, z^{-1}\right\}\left[\frac{1}{t_{-}}\right]$, because $t_{-}(\alpha)=\prod_{i \in \mathbb{N}_{0}}\left(1-\frac{\zeta^{q^{i}}}{\alpha}\right) \neq$ 0 , and hence $C / \mathfrak{m}_{0} C \cong \operatorname{coker}\left(V \rightarrow V^{\prime \prime}\right) \otimes_{\mathbb{F}_{q} \llbracket z \rrbracket} \Omega=(0)$ by using the isomorphism (8.4) again. Finally, in the second case, if $|\alpha| \leq|\zeta|^{s}$, the morphism $\mathcal{O}_{\Omega} \llbracket z \rrbracket \rightarrow \mathcal{O}_{\Omega}((z)) / \mathfrak{m}=\Omega$ factors through $\Omega\left\langle\frac{z}{\zeta^{s}}\right\rangle$, and hence $C / \mathfrak{m}_{0} C \cong \operatorname{coker}\left(V \rightarrow V^{\prime \prime}\right) \otimes_{\mathbb{F}_{q} \llbracket z \rrbracket} \Omega=(0)$ by using the isomorphism (8.5).

Now Proposition 8.3 shows that $\mathcal{G}:=\operatorname{Isom}^{\otimes}\left(\omega^{\circ} \otimes_{\mathbb{F}_{q}((z))} \mathcal{O}_{\Omega}((z)), M \otimes_{\mathcal{O}_{\Omega} \llbracket z \rrbracket} \mathcal{O}_{\Omega}((z))\right)$ is a $G$-torsor over $\mathcal{O}_{\Omega}((z))$ for the fpqc-topology, and hence for the étale topology, because $G$ is smooth. By [3, Proposition 11.5], the $G$-torsor $\mathcal{G}$ over $\mathcal{O}_{\Omega}((z))$ is trivial.

4. From the triviality of $\mathcal{G}$ and from the isomorphism $(8.5)$ we obtain an $\mathcal{O}_{\Omega}((z))$ rational, respectively $\Omega\left\langle\frac{z}{\zeta^{s}}\right\rangle$-rational, tensor isomorphism $\theta: \omega^{\circ} \otimes_{\mathbb{F}_{q}}((z)) \mathcal{O}_{\Omega}((z)) \stackrel{\sim}{\longrightarrow}$ $M \otimes_{\mathcal{O}_{\Omega} \llbracket z \rrbracket} \mathcal{O}_{\Omega}((z))$, respectively $\theta_{2}: \omega_{\mathbb{F}_{q} \llbracket z \rrbracket}^{\circ} \otimes_{\mathbb{F}_{q} \llbracket z \rrbracket} \Omega\left\langle\frac{z}{\zeta^{s}}\right\rangle \stackrel{\sim}{\longrightarrow} M \otimes_{\mathcal{O}_{\Omega} \llbracket z \rrbracket} \Omega\left\langle\frac{z}{\zeta^{s}}\right\rangle$, which we fix in the sequel. Over $\Omega\left\langle\frac{z}{\zeta^{s}}\right\rangle\left[z^{-1}\right]$ the composition $u_{\Omega}^{\prime \prime}:=\theta^{-1} \circ \theta_{2}$ corresponds to an element $u_{\Omega}^{\prime \prime} \in G\left(\Omega\left\langle\frac{z}{\zeta^{s}}\right\rangle\left[z^{-1}\right]\right) \subset G(\Omega((z)))=L G(\Omega)$. The isomorphisms

$$
\begin{aligned}
\theta_{V}^{-1} \circ \tau_{M_{V}} \circ \sigma^{*} \theta_{V}: & V \otimes \mathcal{O}_{\Omega} \llbracket z \rrbracket\left[\frac{1}{z(z-\zeta)}\right] \stackrel{\sim}{\longrightarrow} \sigma^{*} M_{V}\left[\frac{1}{z(z-\zeta)}\right] \stackrel{\sim}{\longrightarrow} M_{V}\left[\frac{1}{z(z-\zeta)}\right] \\
& \sim V \otimes \mathcal{O}_{\Omega} \llbracket z \rrbracket\left[\frac{1}{z(z-\zeta)}\right]
\end{aligned}
$$

provide an automorphism of the tensor functor $\omega^{\circ} \otimes_{\mathbb{F}_{q}((z))} \mathcal{O}_{\Omega} \llbracket z \rrbracket\left[\frac{1}{z(z-\zeta)}\right]$; that is, an element $A \in G\left(\mathcal{O}_{\Omega} \llbracket z \rrbracket\left[\frac{1}{z(z-\zeta)}\right]\right)=L_{z(z-\zeta)} G\left(\mathcal{O}_{\Omega}\right)$. We consider $A$ as an isomorphism $\tau_{\mathcal{G}}:=A \sigma^{*}: \sigma^{*} L_{z(z-\zeta)} G_{\mathcal{O}_{\Omega}} \stackrel{\sim}{\longrightarrow} L_{z(z-\zeta)} G_{\mathcal{O}_{\Omega}}$ of trivial $L_{z(z-\zeta)} G$-torsors. Likewise, the isomorphisms

$$
\left.\left.\left.\delta_{V} \circ \theta_{V}: V \otimes \mathcal{O}_{\Omega} \llbracket z, z^{-1}\right\}\left[\frac{1}{t_{-}}\right] \stackrel{\sim}{\longrightarrow} M_{V} \otimes_{\mathcal{O}_{\Omega} \llbracket z \rrbracket} \mathcal{O}_{\Omega} \llbracket z, z^{-1}\right\}\left[\frac{1}{t_{-}}\right] \stackrel{\sim}{\longrightarrow} V \otimes \mathcal{O}_{\Omega} \llbracket z, z^{-1}\right\}\left[\frac{1}{t_{-}}\right]
$$

provide an automorphism of the tensor functor $\left.\omega^{\circ} \otimes_{\mathbb{F}_{q}((z))} \mathcal{O}_{\Omega} \llbracket z, z^{-1}\right\}\left[\frac{1}{t_{-}}\right]$; that is, an element $\left.\Delta \in G\left(\mathcal{O}_{\Omega} \llbracket z, z^{-1}\right\}\left[\frac{1}{t_{-}}\right]\right)$. The equalities $\rho_{V}(b) \circ \sigma^{*} \delta_{V}=\delta_{V} \circ \tau_{M_{V}}$ yield the equality $b \cdot \sigma^{*} \Delta=\Delta \cdot A$ in $\left.G\left(\mathcal{O}_{\Omega} \llbracket z, z^{-1}\right\}\left[\frac{1}{t_{-}}\right]\right)$. Finally, the isomorphisms

$$
\theta_{2, V}^{-1} \circ \tau_{M_{V}} \circ \sigma^{*} \theta_{2, V}: V \otimes \Omega\left\langle\frac{z}{\zeta^{q s}}\right\rangle \stackrel{\sim}{\longrightarrow} \sigma^{*} M_{V} \otimes \Omega\left\langle\frac{z}{\zeta^{q s}}\right\rangle \stackrel{\sim}{\longrightarrow} M_{V} \otimes \Omega\left\langle\frac{z}{\zeta^{q s}}\right\rangle \stackrel{\sim}{\longrightarrow} V \otimes \Omega\left\langle\frac{z}{\zeta^{q s}}\right\rangle
$$

provide an automorphism of the tensor functor $\omega_{\mathbb{F}_{q} \llbracket z \rrbracket}^{\circ} \otimes_{\mathbb{F}_{q} \llbracket z \rrbracket} \Omega\left\langle\frac{z}{\zeta^{q s}}\right\rangle$; that is, an element $A^{\prime \prime} \in G\left(\Omega\left\langle\frac{z}{\zeta^{q s}}\right\rangle\right) \subset G(\Omega \llbracket z \rrbracket)=L^{+} G(\Omega)$. It satisfies $u_{\Omega}^{\prime \prime} \cdot A^{\prime \prime}=A \cdot \sigma^{*} u_{\Omega}^{\prime \prime}$. We view $A^{\prime \prime}$ as an isomorphism $\tau^{\prime \prime}:=A^{\prime \prime} \sigma^{*}: \sigma^{*} L^{+} G_{\Omega} \stackrel{\sim}{\longrightarrow} L^{+} G_{\Omega}$ of trivial $L^{+} G$-torsors satisfying $u_{\Omega}^{\prime \prime} \circ \tau^{\prime \prime}=$ $\tau_{\mathcal{G}} \circ \sigma^{*} u_{\Omega}^{\prime \prime}$. 
Moreover, choose a representative $\bar{\gamma} \in G(\Omega((z-\zeta)))$ of $\bar{\gamma} \in \mathcal{H}_{G, \hat{Z}}^{\text {an }}(\Omega) \subset \operatorname{Gr}_{G}^{\mathbf{B}_{\mathrm{dR}}}(\Omega)$. Then the element $h:=A \cdot \sigma^{*} \Delta^{-1} \cdot \bar{\gamma} \in G(\Omega((z-\zeta)))$ satisfies $\rho_{V}(h)=\theta_{V}^{-1} \circ \tau_{M_{V}} \circ \sigma^{*} \delta_{V}^{-1} \circ \rho_{V}(\bar{\gamma})$. In formula (8.3) we computed that this is an automorphism of $V \otimes_{\mathbb{F}_{q} \llbracket z \rrbracket} \Omega \llbracket z-\zeta \rrbracket$. Thus, $h \in$ $G(\Omega \llbracket z-\zeta \rrbracket)$, and hence $A^{-1}=\sigma^{*} \Delta^{-1} \cdot \bar{\gamma} \cdot h^{-1}$ lies in $\hat{Z}^{\text {an }}(\Omega)$, because $\sigma^{*} \Delta \in G(\Omega \llbracket z-\zeta \rrbracket)$. Therefore, the pair $\left(L G_{\mathcal{O}_{\Omega}}, \tau_{\mathcal{G}}\right)$ consisting of the trivial $L G$-torsor over $\mathcal{O}_{\Omega}$ associated with $\mathcal{G}$ and $\tau_{\mathcal{G}}:=A \sigma^{*}$, as well as the pair $\left(\mathcal{G}_{\Omega}^{\prime \prime}, \tau^{\prime \prime}\right)$ consisting of the trivial $L^{+} G$-torsor $\mathcal{G}_{\Omega}^{\prime \prime}:=$ $L^{+} G_{\Omega}$ over $\Omega$ with $\tau^{\prime \prime}:=A^{\prime \prime} \sigma^{*}$, together with $u_{\Omega}^{\prime \prime}$ satisfy the hypothesis of Lemma 7.9 with $B=\mathcal{O}_{\Omega}$ and $L=\Omega$. We apply this lemma. Because every finitely generated ideal of $\mathcal{O}_{\Omega}$ is principal, $Y=\operatorname{Spec} \mathcal{O}_{\Omega}$. We obtain a local $G$-shtuka $\underline{\mathcal{G}}^{\prime \prime}=\left(\mathcal{G}^{\prime \prime}, \tau_{\mathcal{G}^{\prime \prime}}\right)$ over $\mathcal{O}_{\Omega}$ bounded by $\hat{Z}^{-1}$ and an isomorphism $u^{\prime \prime}:\left(L \mathcal{G}^{\prime \prime}, \tau_{\mathcal{G}^{\prime \prime}}\right) \stackrel{\sim}{\longrightarrow}\left(L G_{\mathcal{O}_{\Omega}}, \tau_{\mathcal{G}}\right)$ of $L G$-torsors over $\mathcal{O}_{\Omega}$ satisfying $u^{\prime \prime} \circ \tau_{\mathcal{G}^{\prime \prime}}=\tau_{\mathcal{G}} \circ \sigma^{*} u^{\prime \prime}$. The reduction modulo $\zeta$ of $\Delta^{\prime \prime}:=\Delta \circ u^{\prime \prime}$ provides a quasi-isogeny $\underline{\mathcal{G}}_{\mathcal{O}_{\Omega} /(\zeta)}^{\prime \prime} \rightarrow \underline{\mathbb{G}}_{0, \mathcal{O}_{\Omega} /(\zeta)}$, because $\Delta \circ u^{\prime \prime} \circ \tau_{\mathcal{G}^{\prime \prime}}=\Delta \cdot A \circ \sigma^{*} u^{\prime \prime}=b \circ \sigma^{*}\left(\Delta \circ u^{\prime \prime}\right)$. This yields an $\mathcal{O}_{\Omega^{-} \text {-valued point }}\left(\underline{\mathcal{G}}^{\prime \prime}, \bar{\Delta}^{\prime \prime}\right) \in \breve{\mathcal{M}}_{\mathbb{G}_{0}}^{\hat{\boldsymbol{Z}}^{-1}}\left(\mathcal{O}_{\Omega}\right)$. Its image under $\breve{\pi}$ is computed as $\breve{\pi}\left(\underline{\mathcal{G}}^{\prime \prime}, \bar{\Delta}^{\prime \prime}\right)=b^{-1} \Delta^{\prime \prime} \cdot G(\Omega \llbracket z-\zeta \rrbracket)$ with $b^{-1} \Delta^{\prime \prime}=b^{-1} \Delta \circ u^{\prime \prime}=\sigma^{*} \Delta \cdot A^{-1} \cdot u^{\prime \prime}=\bar{\gamma} \cdot h^{-1} u^{\prime \prime}$. Because $h, u^{\prime \prime} \in G(\Omega \llbracket z-\zeta \rrbracket)$, this shows that $\breve{\pi}\left(\underline{\mathcal{G}}^{\prime \prime}, \bar{\Delta}^{\prime \prime}\right)=\bar{\gamma}$ and finally (a) is proved.

To prove (b), fix a representation $\rho \in \operatorname{Rep}_{\mathbb{F}_{q}((z))} G$. By Remark 7.3, the rational Tate module $\check{V}_{\underline{\mathcal{G}}}(\rho)$ is a local system of $\mathbb{F}_{q}((z))$-vector spaces on $\breve{\mathcal{M}}:=\left(\breve{\mathcal{M}}_{\mathbb{\mathbb { G }}_{0}}^{\hat{Z}^{-1}}\right)^{\text {an }}$. In order that it descends to a local system on $\breve{\pi}\left(\left(\breve{\mathcal{M}}_{\mathbb{\mathbb { G }}_{0}}^{\hat{Z}^{-1}}\right)^{\text {an }}\right)=: \operatorname{im}(\breve{\pi})$, it suffices by [31, Definition 4.1] to show that

(i) $\breve{\pi}: \breve{M} \rightarrow \operatorname{im}(\breve{\pi})$ is a covering for the étale topology and

(ii) there is a descent datum $\psi: p r_{1}^{*} \check{V}_{\underline{\mathcal{G}}}(\rho) \stackrel{\sim}{\longrightarrow} p r_{2}^{*} \check{V}_{\underline{\mathcal{G}}}(\rho)$ over $\breve{\mathcal{M}} \times_{\text {im }(\breve{\pi})} \breve{\mathcal{M}}$ where $p r_{i}$ : $\breve{\mathcal{M}} \times \operatorname{im(\breve {\pi })} \breve{\mathcal{M}} \rightarrow \breve{\mathcal{M}}$ is the projection onto the $i$ th factor, such that $\psi$ satisfies the

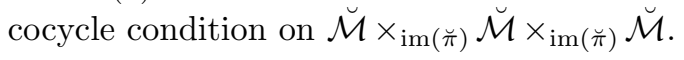

Statement (i) follows from Proposition 6.10. To prove (ii), let $L$ and $B$ be as introduced before Lemma 7.6 and let $\mathcal{X}=\operatorname{Spf} B$ and $X=\mathcal{X}^{\text {an }}=\operatorname{BSpec}\left(B\left[\frac{1}{\zeta}\right]\right)$. Consider an $X$-valued point of $\breve{\mathcal{M}} \times_{\operatorname{im}(\breve{\pi})} \breve{\mathcal{M}}$ and its image in $\breve{\mathcal{M}} \times_{\breve{E}} \breve{\mathcal{M}}$. By [18, Theorem 4.1], this $X$-valued point is induced by two Spf $B$-valued points $(\underline{\mathcal{G}}, \bar{\delta})$ and $\left(\underline{\mathcal{G}^{\prime}}, \bar{\delta}^{\prime}\right)$ of $\breve{\mathcal{M}}_{\underline{\mathbb{G}}_{0}}^{\hat{Z}^{-1}}$ with $\breve{\pi}(\underline{\mathcal{G}}, \bar{\delta})=\breve{\pi}\left(\underline{\mathcal{G}}^{\prime}, \bar{\delta}^{\prime}\right)$ in $\breve{\mathcal{H}}_{G, \hat{Z}, b}^{a}(X)$ possibly after replacing $\operatorname{Spf} B$ by an affine covering of an admissible formal blowing-up; see the explanations before Lemma 6.2 for more details. Now Proposition 7.8 (together with Proposition 5.3) yields a canonical isomorphism $\psi: p r_{1}^{*} \check{V}_{\underline{\mathcal{G}}}(\rho) \stackrel{\sim}{\longrightarrow} p r_{2}^{*} \check{V}_{\underline{\mathcal{G}}}(\rho)$ of local systems of $\mathbb{F}_{q}((z))$-vector spaces over $X$ which is functorial in $\rho$ and satisfies the cocycle condition by canonicity. Therefore, $\check{V}_{\mathcal{G}}(\rho)$ descends to a local system of $\mathbb{F}_{q}((z))$-vector spaces on $\breve{\pi}\left(\left(\breve{\mathcal{M}}_{\underline{\mathbb{G}}_{0}}^{\hat{Z}^{-1}}\right)\right.$ an $)$. Clearly, $\check{V}_{\underline{\mathcal{G}}}: \rho \mapsto \check{V}_{\underline{\mathcal{G}}}(\rho)$ is a tensor functor. The isomorphism $\Phi_{j}: j^{*} \underline{\mathcal{G}} \stackrel{\sim}{\longrightarrow} \underline{\mathcal{G}}$ from Remark 7.17 yields a canonical $J_{b}\left(\mathbb{F}_{q}((z))\right)$-linearisation $\check{V}_{\Phi_{j}}: j^{*} \check{V}_{\underline{\mathcal{G}}}=\check{V}_{j^{*} \underline{\mathcal{G}}} \stackrel{\sim}{\sim} \check{V}_{\underline{\mathcal{G}}}$ on $\check{V}_{\underline{\mathcal{G}}}$ over $\breve{\mathcal{M}}_{\underline{\mathbb{G}}_{0}}^{\hat{Z}^{-1}}$ that descends to $\breve{\pi}\left(\left(\breve{\mathcal{M}}_{\underline{\mathbb{G}}_{0}}^{\hat{Z}^{-1}}\right)\right.$ an $)$ because the period morphism $\breve{\pi}$ is $J_{b}\left(\mathbb{F}_{q}((z))\right)$-equivariant by Remark 6.5.

Let $\rho: G \rightarrow \mathrm{GL}_{r}$ be in $\operatorname{Rep}_{\mathbb{F}_{q} \llbracket z \rrbracket} G$. By [50, Proposition 2.4.4] the pullback to $\breve{\mathcal{M}}_{\mathbb{G}_{0}}^{\hat{Z}^{-1}}$ under $\breve{\pi}$ of the $\sigma$-bundle $\underline{\mathcal{F}}_{b}(\rho)$ over $\breve{\pi}\left(\left(\breve{\mathcal{M}}_{\underline{\underline{G}}_{0}}^{\hat{Z}^{-1}}\right)\right.$ an from Remark 5.6 is canonically isomorphic to 
$\underline{M}_{\rho} \otimes \mathcal{O}_{\breve{\mathcal{M}}_{\underline{\underline{\underline{G}}}_{0}}^{\hat{z}-1}}\left\langle\frac{z}{\zeta^{s}}, z^{-1}\right\}$ where $\underline{M}_{\rho}$ is the local shtuka over $\breve{\mathcal{M}}_{\underline{\mathbb{G}}_{0}}^{\hat{Z}^{-1}}$ associated with the local $\mathrm{GL}_{r}$-shtuka $\rho_{*} \underline{\mathcal{G}}^{\text {univ }}$ obtained from the universal local $G$-shtuka $\underline{\mathcal{G}}^{\text {univ }}$ over $\breve{\mathcal{M}}_{\mathbb{G}_{0}}^{\hat{Z}^{-1}}$. This isomorphism is functorial in $\rho$ and compatible with tensor products and pullback under the action of $j \in J_{b}\left(\mathbb{F}_{q}((z))\right)$ because the period morphism $\breve{\pi}$ is $J_{b}\left(\mathbb{F}_{q}((z))\right)$-equivariant. Descending it to $\breve{\pi}\left(\left(\breve{\mathcal{M}}_{\mathbb{G}_{0}}^{\hat{Z}^{-1}}\right)\right.$ an $)$ and taking $\tau$-invariants yields a canonical isomorphism of tensor functors $\alpha: \underline{\mathcal{V}}_{b} \stackrel{\sim}{\sim} \check{V}_{\underline{\mathcal{G}}}$, which satisfies $\alpha \circ \varphi_{j}=\check{V}_{\Phi_{j}} \circ j^{*} \alpha$ where $\varphi_{j}: j^{*} \underline{\mathcal{V}}_{b} \stackrel{\sim}{\longrightarrow} \underline{\mathcal{V}}_{b}$ is the linearisation from Theorem 5.7.

(c) We fix a geometric base point $\bar{\gamma}$ of $\breve{\pi}\left(\left(\breve{\mathcal{M}}_{\mathbb{G}_{0}}^{\hat{Z}^{-1}}\right)\right.$ an $)$ and consider the canonical family

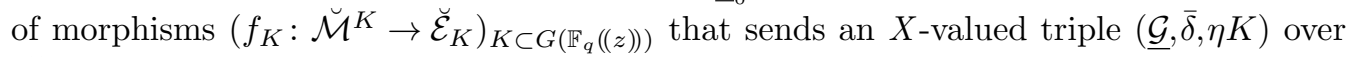
$\breve{\mathcal{M}}^{K}$ from Corollary 7.13 with

$$
\eta K \in \operatorname{Triv}_{\underline{\mathcal{G}}, \bar{\gamma}}\left(\mathbb{F}_{q}((z))\right) / K=\operatorname{Isom}^{\otimes}\left(\omega^{\circ}, \text { forget } \circ \omega_{\bar{\gamma}} \circ \check{V}_{\underline{\mathcal{G}}}\right)\left(\mathbb{F}_{q}((z))\right) / K
$$

to the $X$-valued point of $\breve{\mathcal{E}}_{K}$ given by the $K$-orbit $\beta K \in \operatorname{Isom}^{\otimes}\left(\omega^{\circ}\right.$, forget $\circ \omega_{\bar{\gamma}} \circ$ $\left.\underline{\mathcal{V}}_{b}\right)\left(\mathbb{F}_{q}((z))\right) / K$ of tensor isomorphisms where $\beta:=\left(\right.$ forget $\left.\circ \omega_{\bar{\gamma}}\right)(\alpha)^{-1} \circ \eta$; see Remarks 5.8 and $5.5(\mathrm{a})$. The map $f_{K}$ does not depend on the chosen base point $\bar{\gamma}$ by [31, Theorem $2.9]$ and is thus defined on all connected components of the $\breve{\mathcal{M}}^{K}$. The family $\left(f_{K}\right)_{K}$ is equivariant for the Hecke action of $G\left(\mathbb{F}_{q}((z))\right)$ on both towers defined in (5.5) and (7.5). For any algebraically closed complete extension $\Omega$ of $\breve{E}$, the morphism $f_{K}$ is bijective on $\Omega$-valued points because the fibres of $\breve{\mathcal{M}}^{K}(\Omega)$ and $\breve{\mathcal{E}}_{K}(\Omega)$ over a fixed $\Omega$-valued point of $\breve{\pi}\left(\left(\breve{\mathcal{M}}_{\mathbb{\underline { G }}_{0}}^{\hat{Z}^{-1}}\right)^{\text {an }}\right)$ are both isomorphic to the quotient $G\left(\mathbb{F}_{q}((z))\right) / K$ by Remark $5.5(\mathrm{a})$ and Proposition 7.16. Hence, $f_{K}$ is quasi-finite by [8, Proposition 3.1.4]. Because $\breve{\mathcal{M}}^{K}$ and $\breve{\mathcal{E}}_{K}$ are étale over $\breve{\pi}\left(\left(\breve{\mathcal{M}}_{\mathbb{G}_{0}}^{\hat{Z}^{-1}}\right)^{\text {an }}\right)$, the morphisms $f_{K}$ are étale by [8, Corollary 3.3.9] and hence isomorphisms by [51, Proposition A.4].

To prove that the $f_{K}$ are $J_{b}\left(\mathbb{F}_{q}((z))\right)$-equivariant, we must show that the upper 'rectangle' in the diagram

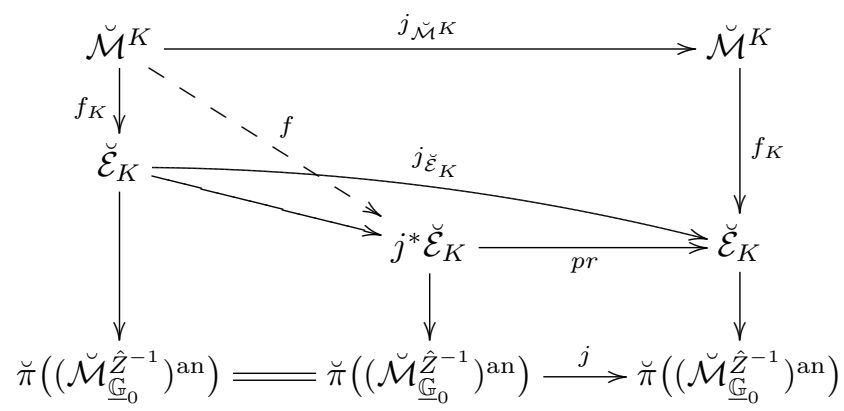

is commutative; that is, $f_{K} \circ j_{\breve{\mathcal{M}}^{K}}=j_{\breve{\mathcal{E}}_{K}} \circ f_{K}$ holds. Recall from Remark 7.17 that the action $j_{\breve{\mathcal{M}}^{K}}: \breve{\mathcal{M}}^{K} \rightarrow \breve{\mathcal{M}}^{K}$ of $j \in J_{b}\left(\mathbb{F}_{q}((z))\right)$ is defined on the universal objects by

$$
j^{*}\left(\underline{\mathcal{G}}^{\text {univ }}, \bar{\delta}^{\text {univ }}, \eta^{\text {univ }} K\right)=\left(j^{*} \underline{\mathcal{G}}^{\text {univ }}, j \circ \bar{\delta}^{\text {univ }} \circ\left(\Phi_{j} \bmod \zeta\right), f \operatorname{forget}\left(\check{V}_{\Phi_{j}, \bar{x}}^{-1}\right) \circ \eta^{\text {univ }} K\right) .
$$


This triple on the source of the morphism $j_{\breve{\mathcal{M}}^{K}}: \breve{\mathcal{M}}^{K} \rightarrow \breve{\mathcal{M}}^{K}$ is mapped under the dashed arrow $f$ to $\left(\right.$ forget $\left.\circ \omega_{\bar{\gamma}}\right)\left(j^{*} \alpha\right)^{-1} \circ \operatorname{forget}\left(\check{V}_{\Phi_{j}, \bar{x}}^{-1}\right) \eta K=\left(\right.$ forget $\left.\circ \omega_{\bar{\gamma}}\right)\left(\varphi_{j}^{-1} \circ \alpha^{-1}\right) \circ \eta K$. Likewise, the image $f_{K}\left(\mathcal{G}^{\text {univ }}, \bar{\delta}^{\text {univ }}, \eta^{\text {univ }} K\right)=\left(\right.$ forget $\left.\circ \omega_{\bar{\gamma}}\right)(\alpha)^{-1} \circ \eta K$ on $\breve{\mathcal{E}}_{K}$ of the universal object on $\breve{\mathcal{M}}^{K}$ is mapped by [51, Formula $\left.(2.11)\right]$ to $\left(\right.$ forget $\left.\circ \omega_{\bar{\gamma}}\right)\left(\varphi_{j}^{-1}\right) \circ($ forget $\circ$ $\left.\omega_{\bar{\gamma}}\right)(\alpha)^{-1} \circ \eta K$ on $j^{*} \breve{\mathcal{E}}_{K}$. This proves the commutativity of the upper 'rectangle' and the $J_{b}\left(\mathbb{F}_{q}((z))\right)$-equivariance of the isomorphism $f_{K}$.

Corollary 8.5. Every point $x \in \breve{\mathcal{M}}^{K}$ has an affinoid neighbourhood $U$ that is finite étale over its image $\breve{\pi}(U)$ in $\breve{\mathcal{H}}_{G, \hat{Z}, b}^{a}$. This image $\breve{\pi}(U)$ is an affinoid subspace of the projective variety $\breve{\mathcal{H}}_{G, \hat{Z}}$. In particular, $\breve{\mathcal{M}}^{K}$ is quasi-algebraic over $\breve{E}$; compare [36, Définition 4.1.11].

Proof. Note that the affinoid neighbourhoods of $\breve{\pi}(x)$ in $\breve{\mathcal{H}}_{G, \hat{Z}, b}^{a}$ form a basis of neighbourhoods of $\breve{\pi}(x)$ by [7, p. 48]. By Theorem 8.1(c) and by the definition of an étale covering space, the point $\breve{\pi}(x)$ therefore has an affinoid neighbourhood $V$ in $\breve{\mathcal{H}}_{G, \hat{Z}, b}^{a}$ such that $\breve{\pi}^{-1}(V)$ is a disjoint union of $\breve{E}$-analytic spaces, each of which is mapping finitely étale to $V$. We can thus take $U$ as the connected component of $\breve{\pi}^{-1}(V)$ containing $x$.

Example 8.6. We exhibit a case in which the tensor functor $M: \operatorname{Rep}_{\mathbb{F}_{q} \llbracket z \rrbracket} G \rightarrow$ $\operatorname{FMod}_{\mathcal{O}_{\Omega} \llbracket z \rrbracket}, V \mapsto M_{V}$ used in the proof of Theorem 8.1(a) does not come from a $G$ torsor over $\mathcal{O}_{\Omega} \llbracket z \rrbracket$. This is also the function field analogue of [89, Remark 5.2.9]. Consider the nonreductive group scheme $G=\mathbb{G}_{a} \rtimes \mathbb{G}_{m}$ over $\mathbb{F}_{q} \llbracket z \rrbracket$ and its representations $\rho: G \stackrel{\sim}{\longrightarrow}\left\{\left(\begin{array}{ll}1 & 0 \\ * & *\end{array}\right)\right\} \hookrightarrow \mathrm{GL}_{2}$ on $V=\mathbb{F}_{q} \llbracket z \rrbracket^{2}$ and $\rho^{\prime}: G \rightarrow \mathbb{G}_{m}$ on $V^{\prime}=\mathbb{F}_{q} \llbracket z \rrbracket$. They sit in an exact sequence in $\operatorname{Rep}_{\mathbb{F}_{q} \llbracket z \rrbracket} G$

$$
0 \longrightarrow\left(V^{\prime}, \rho^{\prime}\right) \stackrel{\left(\begin{array}{l}
0 \\
1
\end{array}\right)}{\longrightarrow}(V, \rho) \stackrel{(1,0)}{\longrightarrow} \mathbb{1} \longrightarrow 0
$$

where $\mathbb{1}: G \rightarrow\{1\} \subset \mathbb{G}_{m}$ is the trivial representation on $\mathbb{F}_{q} \llbracket z \rrbracket$. Let $b=\left(\begin{array}{cc}1 & 0 \\ 0 & -z\end{array}\right) \in G(\mathbb{F}((z)))$ and $\bar{\gamma}=\left(\begin{array}{cc}1 & 0 \\ 0 & (z-\zeta)^{-1}\end{array}\right) \in G(\Omega((z-\zeta))) / G(\Omega \llbracket z-\zeta \rrbracket)$.

Consider the local $G$-shtuka $\underline{\widetilde{\mathcal{G}}}=\left(\left(L^{+} G\right)_{\mathcal{O}_{\Omega}}, \tau_{\mathcal{G}}=\left(\begin{array}{cc}1 & 0 \\ 0 & \zeta-z\end{array}\right)\right)$ over $\mathcal{O}_{\Omega}$. Recall the functor from $\operatorname{Rep}_{\mathbb{F}_{q} \llbracket z \rrbracket} G$ to the category of local shtukas that assigns to $(V, \rho)$ the local shtuka $\underline{M}_{V}$ associated with the local GL( $V)$-shtuka $\rho_{*} \underline{\widetilde{\mathcal{G}}}$ from Remark 6.4. The underlying $\mathcal{O}_{\Omega} \llbracket z \rrbracket-$ module of $\underline{M}_{V}$ equals $V \otimes_{\mathbb{F}_{q} \llbracket z \rrbracket} \mathcal{O}_{\Omega} \llbracket z \rrbracket$. Applied to $\underline{\widetilde{\mathcal{G}}}$ this functor yields the exact sequence of local shtukas

$$
0 \rightarrow \underline{M}^{\prime}=\left(\mathcal{O}_{\Omega} \llbracket z \rrbracket, \tau_{M^{\prime}}=\zeta-z\right) \rightarrow\left(M^{\prime \prime} \oplus M^{\prime}, \tau=\left(\begin{array}{cc}
1 & 0 \\
0 & \zeta-z
\end{array}\right)\right) \rightarrow \underline{M}^{\prime \prime}=\left(\mathcal{O}_{\Omega} \llbracket z \rrbracket, \tau_{M^{\prime \prime}}=1\right) \rightarrow 0 .
$$

Let $f:=\sqrt[q-1]{-1} \cdot t_{+}$. Then the rational Tate module $\mathcal{V}_{b, \bar{\gamma}}=\check{V}_{z} \underline{\widetilde{\mathcal{G}}}$ is 'generated by' $\left(\begin{array}{ll}1 & 0 \\ 0 & f\end{array}\right)=$ $\left(\begin{array}{ll}1 & 0 \\ 0 & \zeta-z\end{array}\right) \cdot \sigma^{*}\left(\begin{array}{ll}1 & 0 \\ 0 & f\end{array}\right)$; that is, the tensor isomorphism $\tilde{\beta}: \omega^{\circ} \stackrel{\sim}{\longrightarrow} \omega_{b, \bar{\gamma}}$ is given by multiplication with

$$
\rho\left(\begin{array}{ll}
1 & 0 \\
0 & f
\end{array}\right): \omega^{\circ}(V, \rho)=V \stackrel{\sim}{\longrightarrow} \omega_{b, \bar{\gamma}}(V, \rho)=\check{V}_{z} \rho_{*} \underline{\widetilde{\mathcal{G}}}
$$


Now we let $\pi \in \mathcal{O}_{\Omega}$ satisfy $\pi^{q-1}=\zeta$ and we choose a different tensor isomorphism $\beta: \omega^{\circ} \stackrel{\sim}{\longrightarrow} \omega_{b, \bar{\gamma}}$ which is given by multiplication with $\rho\left(\begin{array}{ll}1 & 0 \\ f & z f\end{array}\right)=\rho\left(\left(\begin{array}{ll}1 & 0 \\ 0 & f\end{array}\right) \cdot\left(\begin{array}{ll}1 & 0 \\ 1 & z\end{array}\right)\right)$, where $\left(\begin{array}{ll}1 & 0 \\ 1 & z\end{array}\right) \in G\left(\mathbb{F}_{q}((z))\right)$. The construction in step 2 of the proof of Theorem 8.1(a) with $\beta$ instead of $\tilde{\beta}$ replaces every $\underline{M}_{V}$ by a quasi-isogenous one. We claim that for the sequence (8.7) it yields the upper row in the commutative diagram of local shtukas

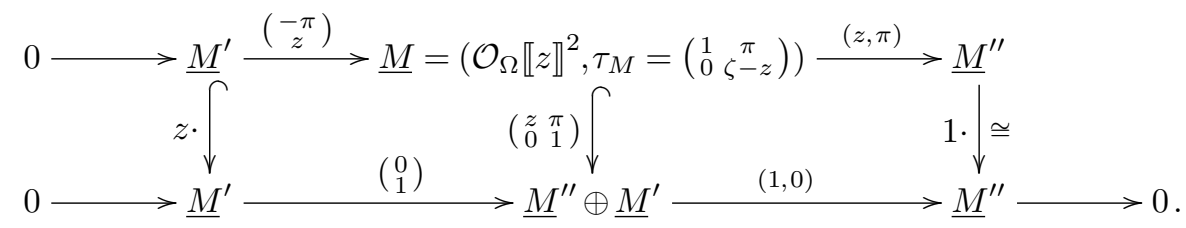

To prove our claim, we note that (8.8) yields over $R:=\mathcal{O}_{\Omega} /(\zeta)$ the diagram

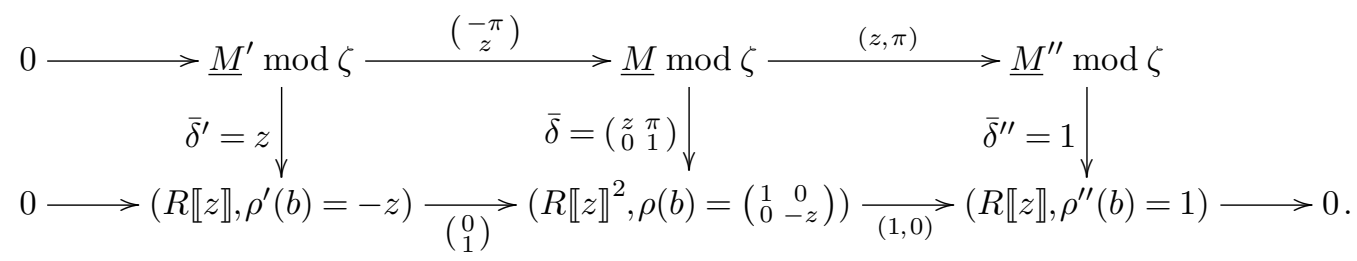

Also, when we consider the element

$$
\Omega \llbracket z \rrbracket^{\times} \ni y:=\frac{1-\pi f}{z}=\frac{1+(z-\zeta) \pi \sigma(f)}{z}=\pi \sigma(f)+\frac{1-\sigma(\pi f)}{z}=\pi \sigma(f)+\sigma(y),
$$

the upper row of (8.8) is right exact on Tate modules, because it induces the following commutative diagram:

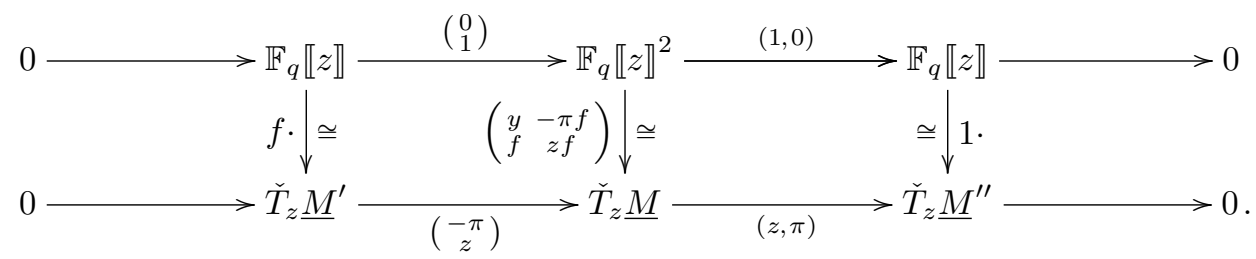

Finally, the vertical quasi-isogeny in the middle of (8.8) induces the following commutative diagram on Tate modules:

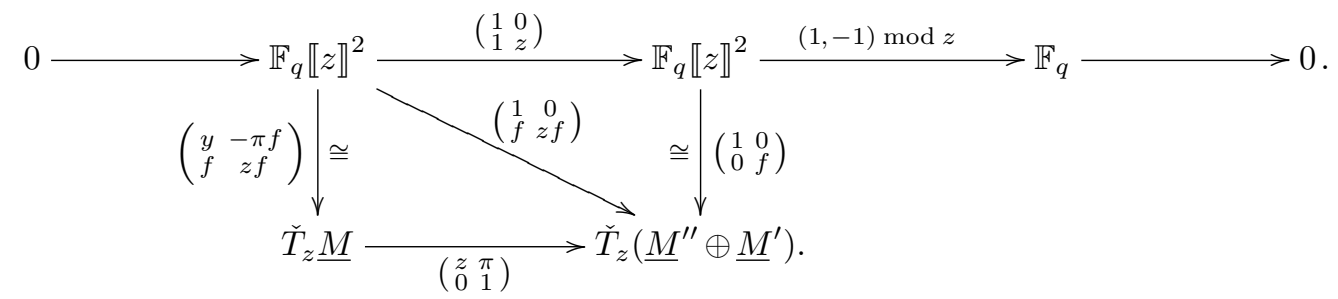

This proves the claim. 
Now, in diagram (8.8), the map $(z, \pi)$ in the upper row is not surjective, and this provides an example where the tensor functor $(V, \rho) \mapsto M_{V}$ is not exact and hence does not come from a $G$-torsor $\mathcal{G}$ over $\mathcal{O}_{\Omega} \llbracket z \rrbracket$. One can also check, although

$$
\left(\begin{array}{ll}
1 & 0 \\
0 & f
\end{array}\right) \cdot\left(\begin{array}{cc}
1 & 0 \\
1 & z
\end{array}\right)=\left(\begin{array}{cc}
1 & 0 \\
f & z f
\end{array}\right)=\left(\begin{array}{cc}
z & \pi \\
0 & 1
\end{array}\right) \cdot\left(\begin{array}{cc}
y & -\pi f \\
f & z f
\end{array}\right) \in \mathrm{GL}_{2}\left(\mathcal{O}_{\Omega}((z))\right) \cdot \mathrm{GL}_{2}\left(\Omega\left\langle\frac{z}{\zeta}\right\rangle\right)
$$

it is not possible to write it as a product in $G\left(\mathcal{O}_{\Omega}((z))\right) \cdot G\left(\Omega\left\langle\frac{z}{\zeta}\right\rangle\right)$. This corresponds to the fact that the quasi-isogeny $\underline{M} \rightarrow \underline{M}^{\prime \prime} \oplus \underline{M}^{\prime}$ does not come from a quasi-isogeny $\underline{\mathcal{G}} \rightarrow \underline{\widetilde{\mathcal{G}}}$ of local $G$-shtukas over $\mathcal{O}_{\Omega}$, because $\underline{\mathcal{G}}$ does not exist. Note also that in terms of the proofs of Proposition 7.8 and Lemma 7.9, it is not possible to extend the étale local $G$-shtuka $\underline{\mathcal{G}}^{\prime \prime}$ over $\Omega$ to $\mathcal{O}_{\Omega}$, because $G$ is not parahoric and $\mathcal{F} \ell_{G}$ and $\underline{\mathcal{M}}_{\underline{\underline{\mathcal{G}}}}$ are not ind-projective.

\section{Cohomology}

In this section we provide basic properties of the cohomology of the towers of moduli spaces. This theory and all of the proofs parallel the one for Rapoport-Zink spaces in [36] to which we also refer for some arguments that go over to our case without modification. Note that instead of the $\breve{E}$-analytic space $\breve{\mathcal{M}}^{K}$ in the sense of Berkovich, we may work with the associated adic space in the sense of Huber by [63, Proposition 8.2.12 and Theorem 8.3.5].

Let $\ell$ be a prime different from the characteristic of $\mathbb{F}_{q}$ and let $\bar{E}$ be the completion of an algebraic closure of $\breve{E}$.

Definition 9.1. We denote by $H_{c}^{\bullet}\left(\breve{\mathcal{M}}^{K} \hat{\otimes}_{\breve{E}} \bar{E}, \mathbb{Q}_{\ell}\right)$ the $\ell$-adic cohomology with compact support of the analytic space $\breve{\mathcal{M}}^{K}$. For short, we denote it by $H_{c}^{\bullet}\left(\breve{\mathcal{M}}^{K}, \mathbb{Q}_{\ell}\right)$ and write $H_{c}^{\bullet}\left(\breve{\mathcal{M}}^{K}, \overline{\mathbb{Q}}_{\ell}\right):=H_{c}^{\bullet}\left(\breve{\mathcal{M}}^{K}, \mathbb{Q}_{\ell}\right) \otimes_{\mathbb{Q}_{\ell}} \overline{\mathbb{Q}}_{\ell}$.

Because the spaces $\breve{\mathcal{M}}^{K}$ are in general only quasi-algebraic, the definition of the cohomology needs some explanation, for which we refer to Fargues [36, §4.1].

These cohomology groups are equipped with the following group actions. The action of $J:=J_{b}\left(\mathbb{F}_{q}((z))\right)$ on $\breve{\mathcal{M}}^{K}$ induces an action on $H_{c}^{\bullet}\left(\breve{\mathcal{M}}^{K}, \mathbb{Q}_{\ell}\right)$ for each $K$. Furthermore, we obtain an action of the Weil group $W_{E}$ of $E$. Indeed, the inertia $\operatorname{subgroup} \operatorname{Gal}\left(\breve{E}^{\mathrm{sep}} / \breve{E}\right)$ acts on the coefficients $\bar{E}$ inducing an action on the cohomology. The action of Frobenius $\sigma \in W_{E}$ is induced by the Weil descent datum of Remark 3.3. As in [36, Remarque 4.4.3], one can show that the induced morphism on cohomology is invertible and thus induces an action of $W_{E}$. Furthermore, for varying $K$ the action by Hecke correspondences induces an action of $G\left(\mathbb{F}_{q}((z))\right)$ on the cohomology groups of the whole tower.

If $\varepsilon: G \rightarrow G^{\prime}$ is a morphism of parahoric group schemes over $\mathbb{F}_{q} \llbracket z \rrbracket$ as in Remarks 3.7, 4.21 and 7.19 with $\varepsilon(K) \subset K^{\prime}$, we obtain a morphism

$$
\varepsilon^{*}: H_{c}^{\bullet}\left(\breve{\mathcal{M}}_{G^{\prime}}^{K^{\prime}}, \mathbb{Q}_{\ell}\right) \longrightarrow H_{c}^{\bullet}\left(\breve{\mathcal{M}}_{G}^{K}, \mathbb{Q}_{\ell}\right)
$$

that is compatible with the actions of the Weil group $W_{E}$, the Hecke action of $G\left(\mathbb{F}_{q}((z))\right)$ that acts on the source via the morphism $G\left(\mathbb{F}_{q}((z))\right) \rightarrow G^{\prime}\left(\mathbb{F}_{q}((z))\right)$ and the action of the group $J_{b}^{G}$ that acts on the source via the morphism $J_{b}^{G} \rightarrow J_{\varepsilon(b)}^{G^{\prime}}$. 
Lemma 9.2. For each $K$, the $J \times W_{E}$-representation $H_{c}^{\bullet}\left(\breve{\mathcal{M}}^{K}, \mathbb{Q}_{\ell}\right)$ is smooth for the action of $J$ and continuous for the action of $W_{E}$.

Proof. As in the arithmetic context (compare [36, Corollaire 4.4.7]), this follows from the fact that the $\breve{\mathcal{M}}^{K}$ are quasi-algebraic by Corollary 8.5 and that $J$ acts continuously on $\breve{\mathcal{M}}^{K}$ by Lemma 7.18, using [36, Corollaires 4.1.19, 4.1.20], two general assertions on the cohomology of Berkovich spaces. Note for this that $J$ has an open pro- $p$ subgroup, namely, $\left\{j \in J \cap L^{+} G(\mathbb{F}): j \equiv 1 \bmod z\right\}$.

Next we are interested in finiteness and vanishing properties of cohomology groups. We need the following finiteness statement for the set of irreducible components.

Lemma 9.3. The action of $J$ on the set of irreducible components of $\breve{\mathcal{M}}_{\mathbb{G}_{0}}^{\hat{Z}^{-1}}$ has only finitely many orbits.

Proof. This is a statement about the underlying reduced subscheme; that is, on the set of irreducible components of the affine Deligne-Lusztig variety $X_{Z^{-1}}(b)$ from (3.2). By [81, Theorem 1.4 and Subsection 2.1] there is a closed subscheme $Y \subset \mathcal{F} \ell_{G}$ of finite type such that for each $g \in X_{Z^{-1}}(b)$ there is a $j \in J$ with $j^{-1} g \in Y$. In other words, $g$ has a representative satisfying $g^{-1} b \sigma^{*}(g)=h^{-1} b \sigma^{*}(h)$ for some (representative of an element) $h=j^{-1} g \in Y \cap X_{Z^{-1}}(b)$. In particular, it is enough to show that $Y \cap X_{Z^{-1}}(b)$ has only finitely many irreducible components. This follows because $Y \cap X_{Z^{-1}}(b)$ is of finite type.

Proposition 9.4. For each compact open subgroup $K \subset G\left(\mathbb{F}_{q} \llbracket z \rrbracket\right)$, the J-representation $H_{c}^{\bullet}\left(\breve{\mathcal{M}}^{K}, \mathbb{Q}_{\ell}\right)$ is of finite type.

Proof. Let $X_{1}, \ldots, X_{t}$ be representatives of the finitely many orbits of the action of $J$ on the set of irreducible components of $\breve{\mathcal{M}}_{\mathbb{G}_{0}}^{\hat{Z}^{-1}}$ (compare Lemma 9.3). Let $K_{0}=G\left(\mathbb{F}_{q} \llbracket z \rrbracket\right)$ and let $U \subset\left(\breve{\mathcal{M}}_{\underline{\mathbb{G}}_{0}}^{\hat{Z}^{-1}}\right)^{\text {an }}=\breve{\mathcal{M}}=\breve{\mathcal{M}}^{K_{0}}$ be the tube over $X:=X_{1} \cup \ldots \cup X_{t}$; that is, the preimage of $X$ under the specialisation map sp from $\left(\breve{\mathcal{M}}_{\mathbb{G}_{0}}^{\hat{Z}^{-1}}\right)^{\text {an }}$ to the underlying topological space of $\breve{\mathcal{M}}_{\mathbb{\mathbb { G }}_{0}}^{\hat{Z}^{-1}}$; see $[11, \S 1]$. If $V_{0}$ is a quasi-compact open subset of $\breve{\mathcal{M}}_{\mathbb{G}_{0}}^{\hat{Z}^{-1}}$ containing $X$, then the complement $Y:=V_{0} \backslash X$ is open and quasi-compact, because $V_{0}^{-\underline{\mathbb{O}_{0}}}$ is Noetherian. Therefore, $V:=s p^{-1}\left(V_{0}\right) \subset \breve{\mathcal{M}}^{K_{0}}$ is a compact neighbourhood of $U$ and $V \backslash U=s p^{-1}(Y)$ is compact, whence $U$ is open in $\breve{\mathcal{M}}^{K_{0}}$. Let $U_{K}:=\breve{\pi}_{K_{0}, K}^{-1}(U) \subset \breve{\mathcal{M}}^{K}$.

Under the fully faithful functor $[8, \S 1.6]$ from strictly $\breve{E}$-analytic spaces to rigid analytic spaces, $U$ and $U_{K}$ correspond to $U^{\text {rig }}=s p^{-1}(X)$ and $U_{K}^{\text {rig }}=\left(\breve{\pi}_{K_{0}, K}^{\text {rig }}\right)^{-1}\left(U^{\text {rig }}\right)$, where $s p:\left(\breve{\mathcal{M}}_{\mathbb{G}_{0}}^{\hat{Z}^{-1}}\right)^{\text {rig }} \rightarrow \breve{\mathcal{M}}_{\mathbb{G}_{0}}^{\hat{Z}^{-1}}$ is the specialisation map [13, (0.2.2.1)]. These are admissible open subspaces of $\left(\breve{\mathcal{M}}_{\underline{\mathbb{G}}_{0}}^{\hat{Z}^{-1}}\right)^{\text {rig }}$ and $\left(\breve{\mathcal{M}}^{K}\right)^{\text {rig }}$. Under the fully faithful functor [63, (1.1.11)] from rigid analytic spaces over $\breve{E}$ to Huber's adic spaces, $U^{\text {rig }}$ and $U_{K}^{\text {rig }}$ correspond to $U^{\text {ad }}=$ $s p^{-1}(X)^{\circ}$ and $U_{K}^{\mathrm{ad}}=\left(\breve{\pi}_{K_{0}, K}^{\mathrm{ad}}\right)^{-1}\left(U^{\mathrm{ad}}\right)$, where $s p:\left(\breve{\mathcal{M}}_{\mathbb{G}_{0}}^{\hat{Z}^{-1}}\right){ }^{\text {ad }} \rightarrow \breve{\mathcal{M}}_{\mathbb{G}_{0}}^{\hat{Z}^{-1}}$ is the specialisation map [63, Proposition 1.9.1] and $s p^{-1}(X)^{\circ}$ denotes the open interior. These are open subspaces of $\left(\breve{\mathcal{M}}_{\underline{\mathbb{G}}_{0}}^{\hat{Z}^{-1}}\right)^{\text {ad }}$ and $\left(\breve{\mathcal{M}}^{K}\right)^{\text {ad }}$. By definition [63, formula $(*)$ on p. 315],

$$
H_{c}^{q}\left(U_{K}^{\mathrm{rig}}, \mathbb{Q}_{\ell}\right):=H_{c}^{q}\left(U_{K}^{\mathrm{ad}}, \mathbb{Q}_{\ell}\right),
$$


and this is a finite-dimensional $\mathbb{Q}_{\ell}$-vector space by [65, Corollaries 5.8 and 5.4]. Because $X$ is proper over $\mathbb{F}$ by Theorem 3.5, $U_{K}^{\text {rig }}$ is partially proper over $\breve{E}$ by [63, Remark 1.3.18]. So $H_{c}^{q}\left(U_{K}, \mathbb{Q}_{\ell}\right)=H_{c}^{q}\left(U_{K}^{\text {rig }}, \mathbb{Q}_{\ell}\right)$ by $[64$, Proposition 1.5]. Note that the proof of [64, Proposition 1.5] only uses that $U_{K}^{\text {rig }}$ is partially proper over $\breve{E}$ and not the stated assumption that $U_{K}$ is closed. (We thank Roland Huber for explaining these arguments to us.)

Let $J^{\prime} \subset J$ be the stabiliser of $U_{K}$, a compact open subgroup. Then the $g \cdot U_{K}$ for $\bar{g} \in J / J^{\prime}$ are a covering of $\breve{\mathcal{M}}^{K}$. We consider the associated spectral sequence for Cech cohomology of [36, Proposition 4.2.2],

$$
E_{1}^{p q}=\bigoplus_{\alpha \subset J / J^{\prime}} H_{c}^{q}\left(U_{K}(\alpha), \mathbb{Q}_{\ell}\right) \Longrightarrow H_{c}^{p+q}\left(\breve{\mathcal{M}}^{K}, \mathbb{Q}_{\ell}\right)
$$

with the sum being over subsets $\alpha$ with $-p+1$ elements and where $U_{K}(\alpha)=\bigcap_{\bar{g} \in \alpha} g \cdot U_{K}$. It is concentrated in degrees $p \leq 0$ and $0 \leq q \leq \operatorname{dim}\left(\breve{\mathcal{M}}_{\mathbb{\mathbb { G }}_{0}}^{\hat{Z}^{-1}}\right)^{\text {an }}$. Furthermore, it is $J$ equivariant where $g \in J$ acts via

$$
g_{!}: H_{c}^{q}\left(U_{K}(\alpha), \mathbb{Q}_{\ell}\right) \rightarrow H_{c}^{q}\left(g \cdot U_{K}(\alpha), \mathbb{Q}_{\ell}\right) .
$$

For $\alpha \subset J / J^{\prime}$, let $J_{\alpha}^{\prime}=\bigcap_{\bar{g} \in \alpha} g J^{\prime} g^{-1}$. By Lemma 7.18, $J_{\alpha}^{\prime} \subset J$ acts continuously on $U_{K}(\alpha)$. Hence, the $H_{c}^{q}\left(U_{K}(\alpha), \mathbb{Q}_{\ell}\right)$ are smooth $J_{\alpha}^{\prime}$-modules. We can rewrite $E_{1}^{p q}$ as compact induction

$$
E_{1}^{p q}=\bigoplus c-\operatorname{Ind}_{J_{\alpha}^{\prime}}^{J} H_{c}^{q}\left(U_{K}(\alpha), \mathbb{Q}_{\ell}\right)
$$

where the sum is now over equivalence classes $\bar{\alpha}$ of subsets $\alpha \subset J / J^{\prime}$ with $-p+1$ elements up to the action of $J$ diagonally on $\left(J / J^{\prime}\right)^{-p+1}$. We claim that there are only finitely many such $\bar{\alpha}$ with $U_{K}(\alpha) \neq \emptyset$.

To show this claim, note that if $A$ is a finite union of irreducible components of $\breve{\mathcal{M}}_{\mathbb{\mathbb { G }}_{0}}^{\hat{Z}^{-1}}$, then the set $\{g \in J: g \cdot A \cap A \neq \emptyset\}$ is compact. In particular, $J^{\prime \prime}=\left\{g \in J: g \cdot U_{K} \cap U_{K} \not \neq \emptyset\right\}$ is compact and contains $J^{\prime}$. Thus, if $\bar{\alpha}=\left\{g_{1}, \ldots, g_{-p+1}\right\}$ is as above with $U_{K}(\alpha) \neq \emptyset$, then for $i \neq j$ we have $g_{i}^{-1} g_{j} \in J^{\prime \prime}$. Modulo the left action of $J$ on the index set we may assume that $g_{-p+1} \in J^{\prime \prime} / J^{\prime}$; hence, all $g_{i}$ are in $J^{\prime \prime} / J^{\prime}$, a finite set. In particular, the index set is finite.

Altogether we obtain that $E_{1}^{p q}$ is a finite sum of compact inductions of finite-dimensional representations and hence a representation of $J$ of finite type. By [12, Remarque 3.12], the category of smooth $J$-modules is locally Noetherian. Because $H_{c}^{p+q}\left(\breve{\mathcal{M}}^{K}, \mathbb{Q}_{\ell}\right)$ has a finite filtration with all subquotients of finite type, it is itself of finite type.

Corollary 9.5. Let $\Pi$ be an admissible representation of $J$. Then for all $K, p$ and $q$

$$
\operatorname{dim}_{\overline{\mathbb{Q}}_{\ell}} \operatorname{Ext}_{J-\text { smooth }}^{p}\left(H_{c}^{q}\left(\breve{\mathcal{M}}^{K}, \overline{\mathbb{Q}}_{\ell}\right), \Pi\right)<\infty .
$$

Proof. This follows from the preceding proposition together with the following fact. Let $H$ be a reductive group over $\mathbb{F}_{q}((z))$ and let $\Pi_{1}$ be a smooth representation of $H$ of finite type and $\Pi_{2}$ an admissible representation. Then $\operatorname{dim}\left(\operatorname{Ext}_{H-\text { smooth }}^{i}\left(\Pi_{1}, \Pi_{2}\right)\right)<\infty$. This fact can be shown in the same way as for $p$-adic groups; compare [36, Lemme 4.4.15]. 
Acknowledgments. We thank the anonymous referee for careful reading and many good comments. We further thank Johannes Anschütz, Bhargav Bhatt, Ofer Gabber, Tom Haines, Jack Hall, Jochen Heinloth, Roland Huber, Eike Lau, Brandon Levin, Stephan Neupert, Timo Richarz, Daniel Schäppi, Peter Scholze and Torsten Wedhorn for helpful discussions and Johannes Anschütz for pointing out an error in an earlier proof of Theorem 8.1(a). The first author acknowledges support of the DFG (German Research Foundation) in the form of SFB 878, Project-ID 427320536 - SFB 1442, and Germany's Excellence Strategy EXC 2044-390685587 'Mathematics Münster: DynamicsGeometry-Structure'. The second author was partially supported by ERC starting grant 277889 'Moduli spaces of local $G$-shtukas'.

\section{References}

[1] J. Alper, Adequate moduli spaces and geometrically reductive group schemes, Algebr. Geom., 1(4) (2014), 489-531. arXiv:1005.2398.

[2] A. Altman and S. Kleiman, Compactifying the Picard scheme, Adv. Math., 35(1) (1980), 50-112. Available at https://core.ac.uk/download/pdf/82272407.pdf.

[3] J. Anschütz, Extending torsors on the punctured $\operatorname{Spec}\left(A_{\text {inf }}\right)$, preprint, http://www. math.uni-bonn.de/people/ja.

[4] M. E. Arasteh Rad and U. Hartl, Local $\mathbb{P}$-shtukas and their relation to global $G$ shtukas, Münster J. Math., 7 (2014), 623-670. arXiv:1302.6143.

[5] M. E. Arasteh Rad and U. HartL, Uniformizing the moduli stacks of global $G$-shtukas, to appear in Int. Math. Res. Notices (2021) preprint, arXiv:1302.6351.

[6] A. Beilinson And V. Drinfeld, Quantization of Hitchin's integrable system and Hecke eigensheaves, preprint, http://www.math.uchicago.edu/ mitya/langlands.html.

[7] V. G. Berkovich, Spectral Theory and Analytic Geometry over Non-Archimedean Fields, Mathematical Surveys and Monographs 33, (American Mathematical Society, Providence, RI, 1990).

[8] V. G. Berkovich, Étale cohomology for non-Archimedean analytic spaces, Publ. Math. I.H.E.S., 78 (1993), 5-161. Available on http://www.wisdom.weizmann.ac.il/ vova/.

[9] V. G. Berkovich, Vanishing cycles for formal schemes, Inv. Math., 115 (1994), 539-571. Available at http://www.wisdom.weizmann.ac.il/ vova/.

[10] V. G. Berkovich, The automorphism group of the Drinfeld half-plane, C.R. Acad. Sci. Paris Sér. I Math., 321(9) (1995), 1127-1132. Available at http://www.wisdom. weizmann.ac.il/ vova/.

[11] V. G. Berkovich, Vanishing cycles for formal schemes II, Invent. Math., 125(2) (1996), 367-390. Available at http://www.wisdom.weizmann.ac.il/ vova/.

[12] J. N. Bernstein, Le "centre" de Bernstein, edited by P. Deligne. Travaux en Cours, Représentations of reductive groups over a local field (Hermann, Paris, 1984), 1-32.

[13] P. Berthelot, Cohomologie Rigide et Cohomologie Rigide à Support Propre, Prépublication 96-03 (IRMA, Rennes, France, 1996). Available at http://perso.univ-rennes1.fr/ pierre.berthelot/.

[14] A. Borel, Linear Algebraic Groups, 2nd Enlarged Edition (Springer, Berlin, 1991).

[15] A. Borel And T. A. Springer, Rationality properties of linear algebraic groups, II, Tôhoku Math. J. (2), 20 1968, 443-497. Available at Project Euclid. 
[16] M. Borovor, Abelian Galois cohomology of reductive groups, Mem. Amer. Math. Soc., 132(626) (1998). Available at http://www.math.tau.ac.il/ borovoi/.

[17] S. Bosch, U. Güntzer and R. Remmert, Non-Archimedean Analysis, Grundlehren 261 (Springer, Berlin, 1984).

[18] S. Bosch and W. Lütkebohmert, Formal and rigid geometry I. Rigid spaces, Math. Ann., 295 (1993), 291-317.

[19] S. Bosch and W. Lütkebohmert, Formal and rigid geometry II. Flattening techniques, Math. Ann., 296 (1993), 403-429.

[20] S. Bosch, W. Lütkebohmert And M. Raynaud, Néron Models, Ergebnisse der Mathematik und ihrer Grenzgebiete (3) 21 (Springer, Berlin, 1990).

[21] S. Bosch, W. Lütkebohmert and M. Raynaud, Formal and rigid geometry III. The relative maximum principle, Math. Ann., 302 (1995), 1-29.

[22] N. Bourbaki, Élements de Mathématique, Algèbre, Chapitres 1 à 3 (Hermann, Paris, 1970).

[23] P. Boyer, Mauvaise réduction des variétés de Drinfeld et correspondance de Langlands locale, Invent. Math., 138(3) (1999), 573-629. Available at http://www.math. univ-paris13.fr/ boyer/.

[24] P. Breutmann, The Carlitz logarithm as a period morphism for local $G$-shtukas, preprint, 2019, arXiv:1907.06456.

[25] F. Bruhat and J. Tits, Groupes réductifs sur un corps local: I. Données radicielles valuées, Inst. Hautes Études Sci. Publ. Math., 41 (1972), 5-251. Available at http://www.numdam.org/numdam-bin/fitem?id=PMIHES_1972__41_5_0.

[26] H. Carayol, Nonabelian Lubin-Tate theory, in Automorphic Forms, Shimura Varieties, and L-Functions, Vol. II (Academic Press, Boston, MA, 1990), 15-39.

[27] M. Chen, Composantes connexes géométriques d'espaces de modules de groupes $p$ divisibles, Ann. l'ENS, 47 (2014), 723-764.

[28] P. Colmez And J.-M. Fontaine, Construction des représentations p-adiques semistables, Invent. Math., 140(1) (2000), 1-43.

[29] B. Conrad, O. Gabber and G. Prasad, Pseudo-reductive Groups, New Mathematical Monographs, 17, (Cambridge University Press, Cambridge, 2010).

[30] J-F. Dat and S. Orlik, M. Rapoport, Period Domains over Finite and p-Adic Fields, Cambridge Tracts in Math., Vol. 183, (Cambridge University Press, 2010).

[31] J. DE Jong, Étale fundamental groups of non-Archimedean analytic spaces, Comp. Math., 97 (1995), 89-118. Available at http://www.math.columbia.edu/ dejong/papers/.

[32] J. DE Jong, et al., Stacks Project, http://stacks.math.columbia.edu/.

[33] P. Deligne And J. Milne, Tannakian categories, in Hodge Cycles, Motives, and Shimura Varieties, LNM 900 (Springer, New York 1982), 101-228. Available at http://www.jmilne.org/math.

[34] M. Demazure and A. Grothendieck, SGA 3: Schémas en Groupes I, II, III, LNM. 151, 152, 153 (Springer, Berlin, 1970). Available at http://library.msri.org/books/sga/ or reedited on http://webusers.imj-prg.fr/ patrick.polo/SGA3/.

[35] V. G. Drinfeld, Coverings of p-adic symmetric domains, Funct. Anal. Appl., 10 (1976), $107-115$.

[36] L. Fargues, Cohomologie des espaces de modules de groupes p-divisibles et correspondances de Langlands locales, Astérisque, 291 (2004), 1-199.

[37] L. Fargues and J.-M. Fontaine, Courbes et fibrés vectoriels en théorie de Hodge $p$ adique, Astérisque, 406 (2018).

[38] J.-M. Fontaine, Modules galoisiens, modules filtrés et anneaux de Barsotti-Tate, Astérisque, 65 (1979), 3-80.

[39] A. Genestier, Espaces symétriques de Drinfeld, Astérisque, 234 (1996). 
[40] A. Genestier and V. Lafforgue, L'isomorphisme des deux tours: une autre approche en égales caractéristiques, Progr. Math., 262 (2008), 327-406. Available at http://www.math.jussieu.fr/ vlafforg/.

[41] A. Genestier And V. Lafforgue, Théorie de Fontaine en égales charactéristiques, Ann. Sci. École Norm. Supér., 44(2) (2011), 263-360. Available at http://www.math.jussieu.fr/ vlafforg/.

[42] D. Goss, Basic Structures of Function Field Arithmetic, Ergebnisse der Mathematik und ihrer Grenzgebiete (3) 35 (Springer, Berlin, 1996).

[43] A. Grothendieck, Fondements de la Géométrie Algébrique, Extraits du Séminaire Bourbaki 1957-1962 (Secrétariat mathématique, Paris, 1962).

[44] A. Grothendieck, Élements de Géométrie Algébrique, Publ. Math. IHES, 4, 8, 11, 17, 20, 24, 28, 32, Bures-Sur-Yvette, 1960-1967; see also Grundlehren, Vol. 166 (Springer, Berlin, 1971).

[45] A. Grothendieck, Revêtements étales et groupe fondamental, LNM 224 (Springer, Berlin-Heidelberg, 1971). arXiv:math/0206203.

[46] L. Gruson, Fibrés vectoriels sur un polydisque ultramétrique, Ann. Sci. École Norm. Sup. (4), 1 (1968), 45-89. Available at http://www.numdam.org/numdam-bin/ fitem?id=ASENS_1968_4_1_1_45_0.

[47] W.J. Haboush, Homogeneous vector bundles and reductive subgroups of reductive algebraic groups, Amer. J. Math., 100(6) (1978), 1123-1137.

[48] T. HAines AND M. RAPOPORT, On parahoric subgroups, appendix to [77]. arXiv:0804.3788.

[49] U. HARTL, A dictionary between Fontaine-theory and its analogue in equal characteristic, J. Number Theory, 129 (2009), 1734-1757. arXiv:math.NT/0607182.

[50] U. Hartu, Period spaces for Hodge structures in equal characteristic, Ann. Math., 173(3) (2011), 1241-1358. arXiv:math.NT/0511686.

[51] U. Hartu, On a conjecture of Rapoport and Zink, Invent. Math., 193 (2013), 627-696. arXiv:math.NT/0605254.

[52] U. Hartl and E. Hellmann, The universal family of semi-stable $p$-adic Galois representations, Algebra Number Theory, 14(5) (2020), 1055-1121. arXiv:math/1312.6371.

[53] U. Hartl And A.-K. JuschKa, Pink's theory of Hodge structures and the Hodge conjecture over function fields, in $t$-Motives: Hodge Structures, Transcendence and Other Motivic Aspects, eds. G. Böckle, D. Goss, U. Hartl and M. Papanikolas (European Mathematical Society Publishing House, Berlin, 2020), 31-182. arxiv:math/1607.01412.

[54] U. Hartl and W. Kim, Local shtukas, Hodge-Pink structures and Galois representations, in t-Motives: Hodge Structures, Transcendence and Other Motivic Aspects, eds. G. Böckle, D. Goss, U. Hartl and M. Papanikolas (European Mathematical Society Publishing House, Berlin 2020), 183-260. arXiv:math/1512.05893.

[55] U. Hartl and R. Pink, Vector bundles with a Frobenius structure on the punctured unit disc, Comp. Math., 140(3) (2004), 689-716. Available at http://www.math. uni-muenster.de/u/urs.hartl/Publikat/.

[56] U. Hartl and E. Viehmann, The Newton stratification on deformations of local $G$ shtukas, J. Reine Angew. Math. (Crelle), 656 (2011), 87-129. arXiv:0810.0821.

[57] U. Hartl and E. Viehmann, Foliations in deformation spaces of local $G$-shtukas, $A d v$. Math., 229 (2012), 54-78. arXiv:1002.2387.

[58] R. Hartshorne, Algebraic Geometry, GTM 52 (Springer, Berlin, 1977).

[59] T. HAusberger, Uniformisations des Variétés de Laumon-Rapoport-Stuhler et conjecture de Drinfeld-Carayol, Ann. Inst. Fourier (Grenoble), 55(4) (2005), 1285-1371.

[60] M. J. Hopkins And B. H. Gross, The rigid analytic period mapping, Lubin-Tate space, and stable homotopy theory, Bull. Amer. Math. Soc., 30(1) (1994), 76-86. 
[61] M. J. Hopkins And B. H. Gross, Equivariant vector bundles on the Lubin-Tate moduli space, Contemp. Math., 158 (1994), 23-88.

[62] B. Howard And G. PAPPas, Rapoport-Zink spaces for spinor groups, Compositio Math., 153(5) (2017), 1050-1118. arXiv:1509.03914.

[63] R. Huber, Étale Cohomology of Rigid Analytic Varieties and Adic Spaces, Aspects of Math., E30 (Friedr. Vieweg \& Sohn, Braunschweig, Germany, 1996). Available at https://link.springer.com/chapter/10.1007/978-3-663-09991-8_9

[64] R. Huber, A comparison theorem for l-adic cohomology, Compositio Math., 112 (1998), $217-235$.

[65] R. Huber, A finiteness result for the compactly supported cohomology of rigid analytic varieties II, Ann. Inst. Fourier (Grenoble), 57(3) (2007), 973-1017.

[66] N. Katz, Travaux de Dwork, Séminaire Bourbaki, 24ème année (1971/1972), Exp. No. 409, pp. 167-200, Lecture Notes in Math., Vol. 317 (Springer, Berlin, 1973).

[67] K. Kedlaya, A p-adic local monodromy theorem, Ann. Math. (2), 160 (2004), 93-184. arXiv:math.AG/0110124.

[68] R. KieHL, Theorem A und B in der nichtarchimedischen Funktionentheorie, Invent. Math., 2 (1967), 256-273.

[69] W. Kim, Rapoport-Zink spaces of Hodge type, Forum of Mathematics, Sigma, 6 (2018), E8.

[70] R. E. Коттwitz, Isocrystals with additional structure, Compositio Math., 56(2) (1985), 201-220.

[71] R. E. Koтtwitz, Isocrystals with additional structure, II, Compositio Math., 109 (1997), $255-339$.

[72] S. LAng, Algebraic groups over finite fields, Amer. J. Math., 78 (1956), 555-563.

[73] G. Laumon, M. Rapoport and U. Stuhler, D-elliptic sheaves and the Langlands correspondence, Invent. Math., 113 (1993), 217-338. Available at http://www.math.uni-bonn. $\mathrm{de} / \mathrm{ag} / \mathrm{alggeom} /$.

[74] M. LAzARD, Les zéros des fonctions analytiques d'une variable sur un corps valué complet, Inst. Hautes Études Sci. Publ. Math., 14 (1962), 47-75. Available at http://www. numdam.org/item?id=PMIHES_1962__14__47_0.

[75] W. LÜtкeвонmert, Formal-algebraic and rigid-analytic geometry, Math. Ann., 286 (1990), 341-371.

[76] S. NEupert, Foliations and the cohomology of moduli spaces of bounded global $G$-shtukas, PhD thesis (TU Munich, Garching, 2016). arXiv:math/1610.05935.

[77] G. PAPpas and M. Rapoport, Twisted loop groups and their affine flag varieties, Adv. Math., 219 (2008), 118-198. arXiv:math/0607130.

[78] M. RAPOPORT AND M. Richartz, On the classification and specialization of $F$-isocrystals with additional structure, Compositio Math., 103 (1996), 153-181.

[79] M. Rapoport And E. Viehmann, Towards a theory of local Shimura varieties, Münster J. Math., 7 (2014), 273-326.

[80] M. Rapoport and T. Zink, Period Spaces for p-Divisible Groups, Ann. Math. Stud., Vol. 141 (Princeton University Press, Princeton, NJ 1996).

[81] M. RAPOPORT AND T. ZINK, A finiteness theorem in the Bruhat-Tits building: an application of Landvogt's embedding theorem, Indag. Mathem., N.S., 10(3) (1999), 449458.

[82] M. Raynaud, Géométrie analytique rigide d'apres Tate, Kiehl, .., Bull. Soc. Math. France, Mémoires, 39/40 (1974), 319-327.

[83] M. Raynaud and L. Gruson, Critères de platitude et de projectivité, Invent. Math., 13 (1971), 1-89. 
[84] T. Richarz, Affine Grassmannians and geometric Satake equivalences, International Mathematics Research Notices IMRN 2016, 3717-3767. arXiv:math/1311.1008.

[85] D. SchёPPI, A characterization of categories of coherent sheaves of certain algebraic stacks, arXiv:1206.2764.

[86] D. SchäPPI, Constructing colimits by gluing vector bundles, preprint, arxiv:1505.04596.

[87] T. Schauch, Weak admissibility of Hodge-Pink lattices in terms of geometric invariant theory, $\mathrm{PhD}$ thesis (University of Muenster, 2014). Available at http://nbn-resolving.de/urn:nbn:de:hbz:6-12349573748.

[88] W. H. Schikhof, Ultrametric Calculus. An Introduction to p-Adic Analysis, Cambridge Studies in Advanced Mathematics 4, (Cambridge University Press, Cambridge, 1984).

[89] P. Scholze And J. Weinstein, Moduli of $p$-divisible groups, Cambridge J. Math., 1(2) (2013), 145-237.

[90] P. Scholze and J. Weinstein, Berkeley lectures on $p$-adic cohomology, Annals of Mathematics Studies 207, 2021, www.math.uni-bonn.de/people/scholze/Berkeley.pdf.

[91] J.-P. SERre, Groupes de Grothendieck des schémas en groupes réductifs déployés, Inst. Hautes Études Sci. Publ. Math., 34 (1968), 37-52. Available at http://www.numdam.org/numdam-bin/fitem?id=PMIHES_1968__34_37_0.

[92] J.-P. SERre, Cohomologie galoisienne, LNM 5 (Springer, Berlin, 1997).

[93] T. A. Springer, Linear Algebraic Groups (Birkhäuser, Boston, MA, 2009).

[94] Y. Taguchi and D. Wan, $L$-functions of $\varphi$-sheaves and Drinfeld modules, J. Amer. Math. Soc., 9(3) (1996), 755-781. Available at http://www2.math.kyushu-u.ac.jp/ $\sim$ taguchi/bib/.

[95] A.C.M. van RoolJ, Non-Archimedean Functional Analysis, Monographs and Textbooks in Pure and Applied Math., Vol. 51 (Marcel Dekker, New York, 1978).

[96] T. Wedhorn, On Tannakian duality over valuation rings, J. Algebra, 282(2) (2004), 575609. Available at http://www2.math.uni-paderborn.de/people/torsten-wedhorn.

[97] J.-P. Wintenberger, Propriétés du groupe tannakien des structures de Hodge $p$-adiques et torseur entre cohomologies cristalline et étale, Ann. Inst. Fourier, 47 (1997), 1289-1334. Available at http://www.numdam.org/item?id=AIF_1997_-47__5_1289_0. 